\title{
Plant cortical microtubule dynamics and cell division plane orientation
}




\section{Thesis committee}

\section{Promotors}

Prof. Dr B.J.G. Scheres

Professor of Plant Developmental Biology

Wageningen University \& Research

Prof. Dr B.M. Mulder

Special professor Theoretical Cell Physics

Wageningen University \& Research

Group Leader Theory of Biomolecular Matter

AMOLF, Amsterdam

\section{Other members}

Prof. Dr H. Hofte, INRA Centre Versailles-Grignon, France

Prof. Dr A. Akhmanova, Utrecht University

Prof. Dr J. van der Gucht, Wageningen University \& Research

Prof. Dr D. Weijers, Wageningen University \& Research

This research was conducted under the auspices of the Graduate School Experimental Plant Sciences 


\title{
Plant cortical microtubule dynamics and cell division plane orientation
}

\author{
Bandan Chakrabortty
}

Thesis

submitted in fulfilment of the requirements for the degree of doctor at Wageningen University

by the authority of the Rector Magnificus,

Prof. Dr A.P.J. Mol,

in the presence of the

Thesis Committee appointed by the Academic Board

to be defended in public

on Tuesday 30 May 2017

at 11 a.m. in the Aula. 
Bandan Chakrabortty

Plant cortical microtubule dynamics and cell division plane orientation, 132 pages.

PhD thesis, Wageningen University, Wageningen, the Netherlands (2017) With references, with summary in English

ISBN: 978-94-6343-182-8

DOI: $10.18174 / 413054$ 


\section{Contents}

\begin{tabular}{lll}
\hline & Introduction & 1
\end{tabular}

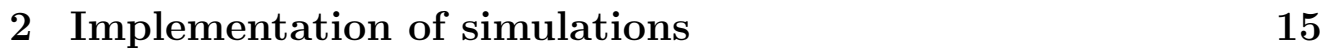

2.1 Processing confocal images for simulation . . . . . . . . . . . 16

2.2 Description of the simulation technique . . . . . . . . . . . . 17

2.2 .1 3D-triangulated surface to 2D-triangles . . . . . . . . 18

2.2 .2 Microtubule dynamics on individual triangles . . . . 19

$2.2 .3 \quad$ Propagating microtubule dynamics . . . . . . . . . . . 22

2.2.4 Mapping microtubule segments from 2D-plane to 3Dpolyhedral surface . . . . . . . . . . . . . 25

2.3 Extension to two dimensional plane . . . . . . . . . . . . . 26

\begin{tabular}{|lll}
3 & Parameterization of simulations & 28 \\
\hline
\end{tabular}

3.1 Input control parameter . . . . . . . . . . . . . . . . . . . 29

3.2 Output order parameter . . . . . . . . . . . . . . . . . . 31

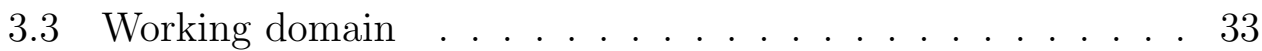

3.4 Influence of triangulation . . . . . . . . . . . . . . . . 34

3.5 Summary $\ldots \ldots \ldots \ldots \ldots$

4 Cortical microtubule order as interplay between shape ef-

fect and stability rules 36

4.1 Contribution of microtubule interactions . . . . . . . . . 37

4.2 Contribution of shape . . . . . . . . . . . . . . . . . . . . 38

$4.3 \quad$ Contribution of edge-catastrophe . . . . . . . . . . . . . . 43

4.4 Contribution of face stability . . . . . . . . . . . . . . . . . 47

4.5 Discussion . . . . . . . . . . . . . . . . . . . 51

5 Explaining the orientation of Arabidopsis embryonic cell

division planes $\quad 52$

$5.1 \quad$ Interplay between shape anisotropy and and edge catastrophe predicts a robust orientation of division plane . . . . . . . 54

5.2 Cell shape anisotropy and edge catastrophe are sufficient to recreate the $b d l$ cell division patterns $\ldots \ldots \ldots \ldots 6$

$5.3 \quad$ Anisotropic growth and/or stress may have role in division plane orientation . . . . . . . . . . . . . . 62

$5.4 \quad$ Nuclear position correlates with the PPB formation site that determines location of division plane . . . . . . . . 65

5.5 Discussion . . . . . . . . . . . . . . . . 67 
6 Division plane reorientation in Arabidopsis root epidermal cells $\quad 69$

6.1 Divisions in normal condition . . . . . . . . . . . . 73

6.2 Divisions under PLT2 induction . . . . . . . . . . . . . 75

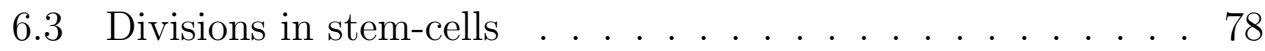

6.4 Quantitative analysis of division planes . . . . . . . . . . . 80

6.5 Discussion . . . . . . . . . . . . . . . . 81

\begin{tabular}{lll}
\hline 7 & General discussion and outlook & 84
\end{tabular}

\begin{tabular}{lr}
\hline Supplementary results & 92
\end{tabular}

S.1 Case study for 1-cell stage bdl embryo of Arabidopsis . . . 92

S.1.1 Default shape simulation . . . . . . . . . . 92

S.1.2 Combined effect of edge-catastrophe and enhanced microtubule stabilization at the basal face . . . . . . 92

S.1.3 Effect of enhanced stabilization of microtubules at the basal face . . . . . . . . . . . . . . . 93

S.1.4 Effect of microtubule stabilizing polar cap . . . . . . 94

S.2 Simulating the PPB formation mechanism . . . . . . . . . . 94

S.3 Sensitivity of microtubule array orientation to embryonic cell shape anisotropy . . . . . . . . . . . . . . . . 95

\begin{tabular}{lr}
\hline Appendix & $\mathbf{9 7}$
\end{tabular}

A.1 Geometric measures on a triangulated shape . . . . . . . . . 97

A.2 Simulation technicalities . . . . . . . . . . . . . . 101

A.3 Order parameter . . . . . . . . . . . . . . 107

\begin{tabular}{ll}
\hline References & 111
\end{tabular}

\begin{tabular}{ll}
\hline English summary & 123
\end{tabular}

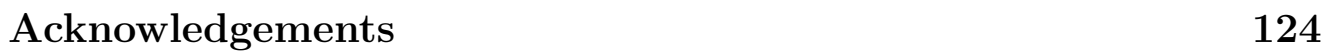




\section{Chapter 1}

\section{Introduction}

Plant cells do not move with respect to each other, causing the morphology of the plant to be determined by the oriented cell division and controlled cell expansion. Therefore, a key issue in plant developmental biology is to understand how directional growth and the subsequent oriented division of cells determine the morphogenesis of the whole organism. In developmental

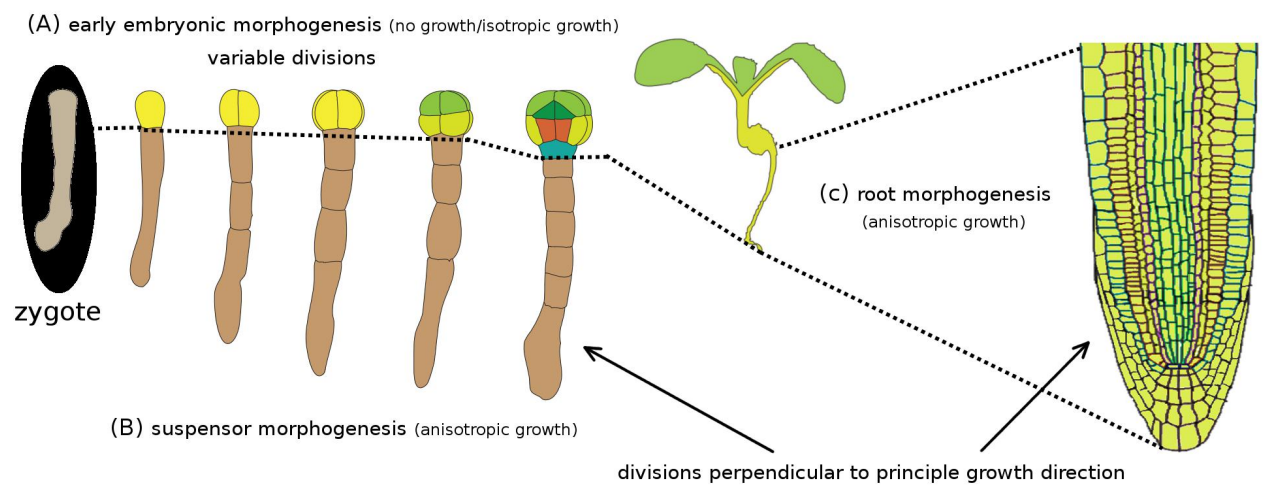

Fig. 1.1. Oriented cell division and plant morphogenesis. Early stages of embryonic development go through highly variable division plane orientation, while almost no growth in cell size/volume. For suspensor/root development, cells go through anisotropic expansion and divide perpendicular to the principle growth direction. Images are adapted from ten Hove et al. (2015) and Smet et al. (2015).

biology, the architecture of both plants and animals is encoded implicitly as a set of growth instructions, that are executed on and by the cellular components and individual cells (Davidson, 2006). The developmental steps affect the morphology of the entire plant. Therefore, the establishment of the correct progenitor cells at the correct embryonic stages and the formation of the basic spatial organisation of the plant body are shielded from the environment. During post-embryonic development, the physiological responses and interactions of the plant with the environment make up a broader biological world that extends beyond the genetically encoded body plan (Scheres and van der Putten, 2017).

During Arabidopsis embryogenesis, particularly the early stages of embryonic development go through highly variable orientation of cell division plane, while almost no growth occurs (see Fig. 1.1. A). However, suspensor cells and later elongating post embryonic cells go through anisotropic expansion, where the divisions are always perpendicular to the principle growth direction (see Fig. 1.1, B and C). Oriented cell division helps to get efficiently structured layers in the embryo and in post-embryonic organs 
for pattern formation, which directs morphogenesis by supporting growth in the right direction (anisotropic cell expansion). Whether for purpose of patterning cell identities or for rendering efficiency to morphogenesis, understanding plant development requires understanding the processes of oriented cell division, controlled cell expansion and their interaction with the formation of a pattern of cell specification.

The work of Turing (1952) has shown that pattern formation can program fields of cells which behave like "excitable media", where the characteristic activities that drive the system into one of several states, were presumed to be chemical. Analogously, morphogenesis can be seen as patterns elaborated by "excitable molecular states of the structural building blocks" that can fall into separate stable states, where each of these states may correspond to a particular morphology. In normal biological pattern formation, a strong selective pressure may exist to maintain one of the different possible morphological patterns. For instance, during the early embryonic development of a species the pattern of cell division may have to be robust to allow correct cell specification mechanisms. In contrast, without such selective pressure, preference for stable pattern is not necessary, i.e patterns of pigmentation stripes in zebra fish or the shells of individual molluscs, which display stochasticity (Meinhardt, 1998).

Over the decades, botanists have derived division rules for oriented cell divisions in plants (Kwiatkowska, 2004). More than a century ago, Hofmeister (1863) proposed that a new cell wall appears perpendicular to the principal growth direction of the cell. Fifteen years later, Sachs (1878) suggested that new cell wall should be inserted perpendicular to existing cell walls. Ten years later, drawing an analogy with soap bubbles, Errera

\section{Geometrical rules}

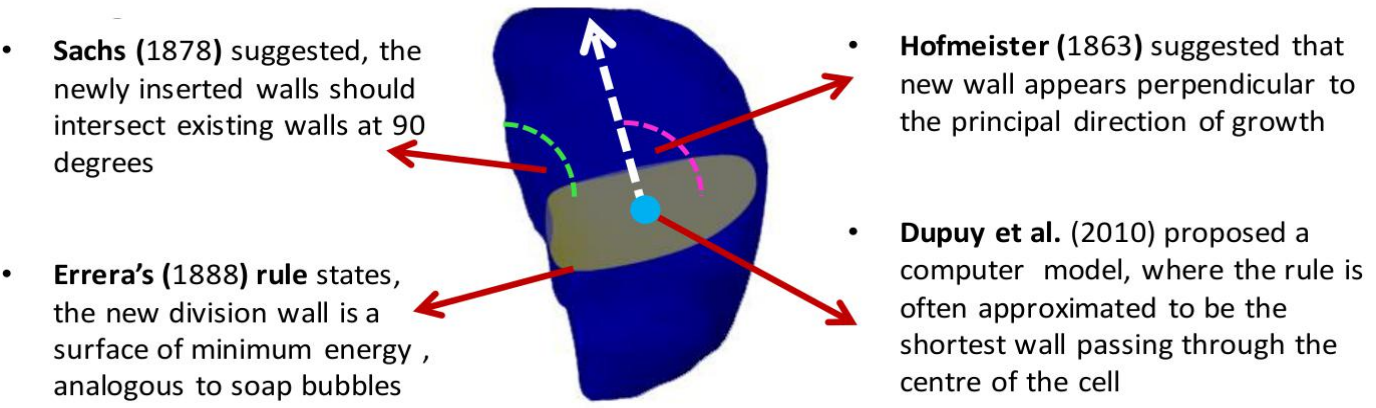

Fig. 1.2. Geometrical rules for cell division in plant cell. In this diagram, we summarized four major rules for cell division proposed by Hofmeister (1863), Sachs (1878), Errera (1888) and Dupuy et al. (2010).

(1888) postulated that the new cell wall will be a surface with minimum surface energy. Following this, Besson and Dumais (2011) have recently included inherent stochasticity of cell division to Errera's rule and derived a 
rule based on the competition between local minima in energy that was able to predict the observed division patterns in a wide range of land plants and algae. They proposed this to be a generic rule for symmetric cell division in plants. Recently in few simulation models, the cell division rule was often approximated to be the wall with minimum circumference, which often in the literature is called shortest wall, passing through the centre of the cell (Dupuy et al., 2010; Smith et al., 2006; Stoma et al., 2008). On top of these existing rules, a stochasticity in the positioning of the cell center turns out to generate more representative cell division patterns (Nakielski, 2000; Sahlin and Jönsson, 2010). However, none of these deterministic rules were able to match all actual cell division patterns. Together, these well known geometrical rules for oriented cell divisions in plants (see Fig. 1.2) give an intuitive framework for rationalizing cell division planes, but the molecular mechanism used by the plant to implement these rules is not yet understood.

In this thesis, we search for the molecular rules that underlie geometrical rules of cell division. It is well known that plant cortical microtubule (CMT) cytoskeleton plays a decisive role in the oriented division of cells. We also can conceptualize microtubules as "excitable medium" forming on the surface of cells. In the absence of microtubule (MT) organizing centers like centrosomes, higher plants establish an ordered array of microtubules at the cell cortex, and the orientation of the division plane follows the orientation of the cortical microtubule array (Jaspersen and Winey, 2004). Besides controlling cell division plane orientation, a long standing debate is whether oriented cortical microtubule arrays have a direct role in controlling cell expansion and cell anisotropy. In the primary cell walls of growing plant cells, the glucose polymer cellulose is assembled into long microfibrils. The rigidity and orientation of these microfibrils control cell expansion through providing a biophysical constraint, to restrict turgor induced cell expansion along the axis perpendicular to the net orientation of the cellulose microfibrils (Lloyd, 2001). During interphase, cortical microtubules are typically coaligned with the cellulose microfibrils (Lucas and Shaw, 2008; Bringmann et al., 2012; Crowell et al., 2009). Based on this co-alignment, it has been speculated that the organization of the interphase cortical microtubules regulates the axis of cell elongation, by guiding the oriented deposition of cellulose microfibrils (Green, 1980). However, recent studies have revealed that cortical microtubule array organization, cellulose microfibril deposition and the orientation of the cell elongation axis share a complex relationship (Baskin, 2001; Sugimoto et al., 2001; Himmelspach et al., 2003; Burk and Ye, 2001). Further attempts towards understanding this complexity have been made in Baskin et al. (2004), where they proposed that anisotropic cell growth is dependent on the degree of global microfibril alignment, while the ordered interphase cortical microtubule arrays play important 


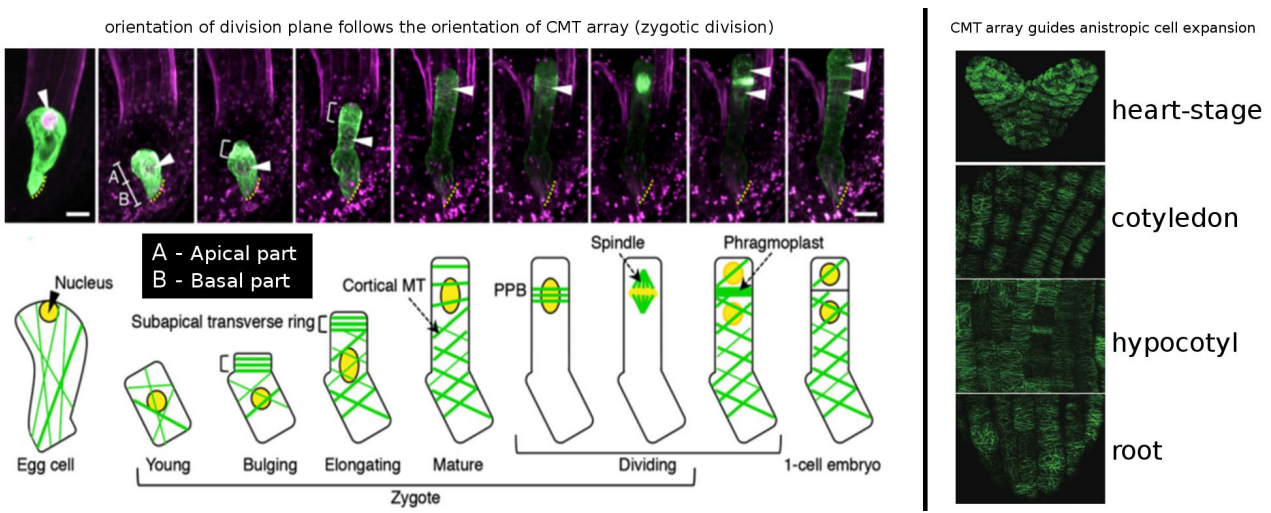

Fig. 1.3. Experimental evidence of: (a) Orientational overlap of CMT array and division plane, and (b) CMT array guiding efficient anisotropic cell expansion. Images are adapted from Wang and Huang (2014) and Kimata et al. (2016) .

role in this alignment process. The intimacy in the relationship between microtubules and cellulose microfibrils became more clear, when Paredez et al. (2006) discovered that cellulose synthase complexes can actually move along microtubules. Genetic evidence of the influence of cellulose synthase activity on cortical microtubule array organization has also been reported (Paredez. et al., 2008). Recently, a proof of concept about the role of the cortical microtubule array in guiding the cellulose synthase complex has been discussed by which the cortical microtubule array can control anisotropic cell expansion (Emons et al., 2007; Lindeboom et al., 2008). In Fig. 1.3, we illustrate the orientational overlap of CMT array and division plane, and also an evidence of CMT array guiding efficient anisotropic cell expansion.

Taken together, mechanisms behind oriented cell division and controlled cell expansion that drive plant morphogenesis are hidden inside the properties allowed by cortical microtubule dynamics. In this thesis, we investigate division plane orientation in plant cells using this as null hypothesis.

\section{Microtubules as individual molecular aggregates}

Microtubules are highly dynamic and filamentous protein polymer aggregates, which form one of the principal components of the plant cytoskeleton. In Fig. 1.4, we explain the dynamics of an individual microtubule. A single microtubule generally consists of 13 linear and parallely arranged protofilaments, assembled around a hollow core (Mandelkow and Mandelkow, 1994). Each protofilament is composed of an array of tubulin dimers, consisting of $\alpha$-tubulin and $\beta$-tubulin monomers. This specific arrangement of the tubulin-protofilaments results in a rigid hollow rod like structure with diameter $\approx 25 \mathrm{~nm}$. Both $\alpha$-tubulin and $\beta$-tubulin can bind to GTP, which promotes polymerization by creating a protective cap. However, shortly after polymerization, a GTP bound to the $\beta$-tubulin of the newly added 

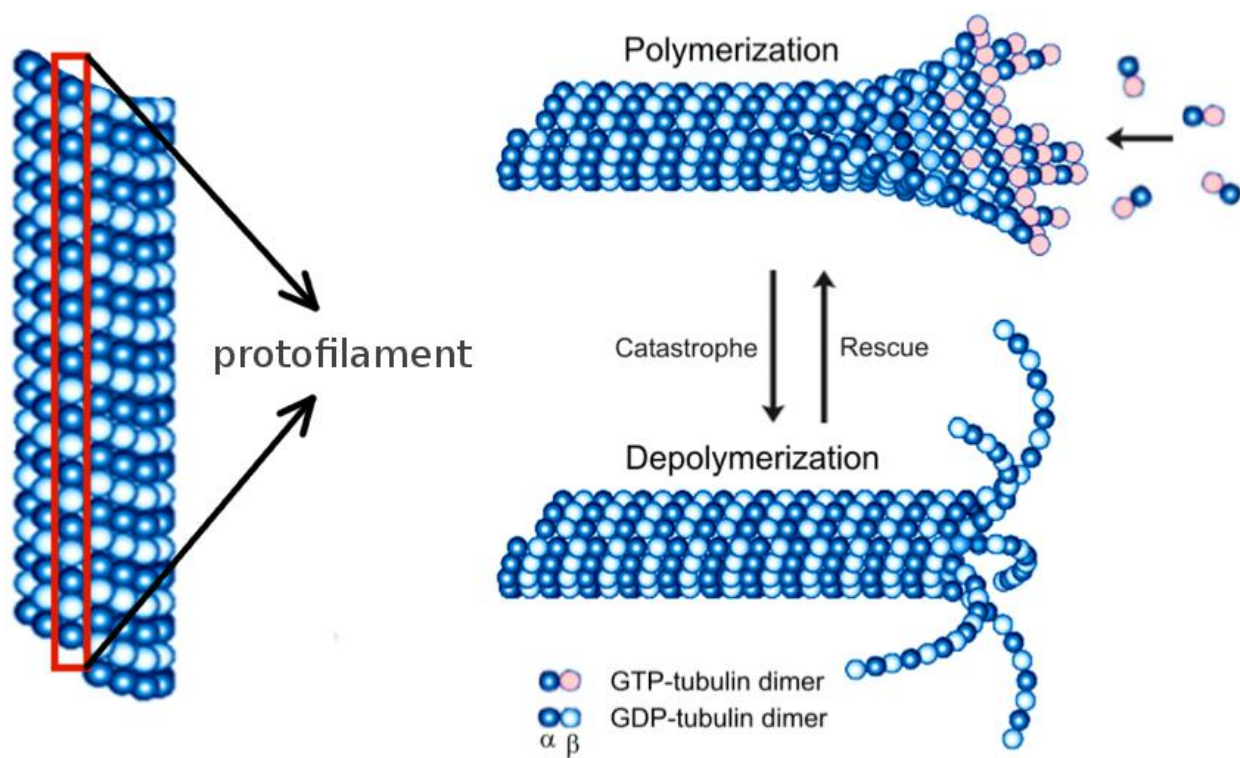

Fig. 1.4. Microtubule structure and intrinsic dynamics. Microtubules are polymers, consisting of linear and parallely arranged protofilaments, where every single protofilament is composed of an array of tubulin dimers ( $\alpha$ and $\beta$ monomers). GTP-bound tubulin promotes polymerisation of a microtubule, resulting microtubule growth. GDP-bound tubulin results in depolymerization that leads to microtubule shrinkage. Microtubule ends can dynamically switch from growing state to shrinking state, called as catastrophe or vice versa, called as rescue. The image is adapted from Dráber et al. (2012).

tubulin dimer is hydrolysed to GDP. This results in a decrease in the binding affinity of the associated tubulin to the adjacent tubulin, leading to depolymerization. Polymerization of tubulin leads to microtubule growth and depolymerization of tubulin leads to microtubule shrinkage. Average growth or shrinkage of a microtubule will be determined by the relative rate of tubulin polymerization to GTP hydrolysis. If at a microtubule end, the GTP-bound tubulin polymerization rate is higher than GTP hydrolysed depolymerization, the microtubule end retains a GTP cap and continues growing. However, if the rate of polymerization slows down, the GTP bound to the $\beta$-tubulin of the associated tubulin dimer will be hydrolysed, resulting in rapid depolymerization and hence shrinking of the microtubule end. Depending on the instantaneous rate of GTP bound tubulin polymerization and GTP hydrolysed depolymerization, a microtubule end can switch from a growing state to a shrinking state or vice versa. Switching of a microtubule end from a growing state to a shrinking state is called catastrophe and switching of a microtubule end from a shrinking state to a growing state is called rescue. This phenomenon of reversible switching of microtubule ends from one state to an other is called dynamic instability. Microtubules have structurally two distinct ends - a minus end, exposing $\alpha$-tubulin, and a plus end, exposing $\beta$-tubulin. On average, GDP bound tubulin is continually lost from the minus end of a microtubule and get replaced by addition of GTP bound tubulin to the plus end of the same microtubule. Thus overall 
growth at the plus end and shrinkage at the minus end seemingly moves a microtubule as a whole. This motion is called treadmilling and has been observed in both in vitro (Rothwell et al., 1985; Panda et al., 1999; Grego et al., 2001) and in vivo studies (Rodionov and Borisy, 1997).

\section{Microtubules on the plant cell cortex}

In plant cells, microtubules can be categorized in two types, the cytoplasmic microtubules that accumulate in a banded pattern during spindle and phragmoplast formation, and the cortical microtubules, which are confined to a thin layer of cytoplasm just inside the plasma membrane of the cell. Fluorescence recovery after photo-bleaching (FRAP) experiments revealed that the microtubules are fixed with respect to the cell surface, confirming microtubules do not translate or rotate as a whole (Shaw et al. 2003; Vos et al., 2003). In the same experiments no evidence was found for motor activity on the microtubules or detachment (reattachment) of microtubules from (to) the cell cortex. This indicates that cortical microtubules remain stably attached to the cell cortex. Electron microscopy has shown a cross-bridging between cortical microtubules and the cell (inner membrane) cortex, indicating the presence of linker proteins that anchor the microtubules to the cell cortex (Hardham and Gunning, 1978). Though the molecular identity of many of these anchor proteins is yet to be revealed (Gardiner et al., 2001; Hashimoto and Kato, 2006; Hamada, 2007; Kirik et al., 2007), two of them were reported to be CLASP (Ambrose and Wasteneys, 2008) and phospholipase D (Dhonukshe et al., 2003). In plant

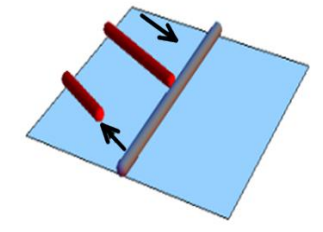

induced-catastrophe

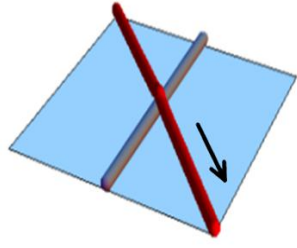

crossover

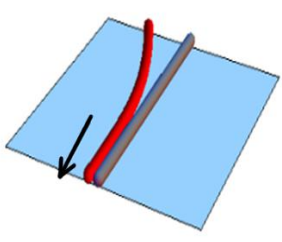

zippering

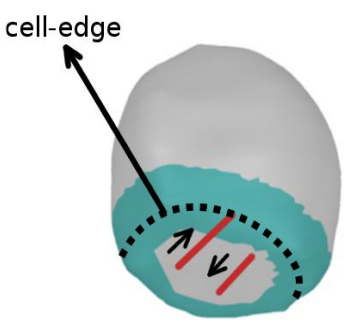

edge-catastrophe

Fig. 1.5. Collision events during microtubule-microtubule interactions and edge-catastrophe during microtubule to cell-edge encounter. A steep angle of collision leads to induced-catastrophe or crossover and a shallow angle of collision leads to zippering. When a microtubule encounters a cell-edge, depending on the degree of curvature at the cell-edge, the microtubule faces a catastrophe. Higher the value of curvature stronger the probability of suffering an edge-catastrophe. Courtesy for the Drawing of the schematic representation: B. M. Mulder (AMOLF).

cells, microtubules do not have a well defined organizing center. Instead, microtubule organizing center activity (via $\gamma$-tubulin complex) is localized at dispersed sites of the cell cortex (Schmit, 2002). This accounts for the dispersed nucleation of cortical microtubules as well. However, detailed microscopic observations have revealed that a large majority of cortical 
microtubules are in fact nucleated from nucleation complexes attached to pre-existing microtubules and at specific angles with respect to the parent microtubules (Chan et al., 2009; Murata et al., 2005). In spite of their fixed attachment to the cell cortex, cortical microtubules do show mobility which is due to treadmilling motion (Shaw et al., 2003; Vos et al., 2004). The presence of treadmilling motion indicates that plant cortical microtubules are not anchored at their minus-ends but must be released from their nucleation sites. Attachment of cortical microtubules to the cell cortex through the anchoring proteins effectively confines them to a two dimensional surface. Due to this two dimensional confinement, microtubules interact with each other via collisions, which occur when the polymerizing tip of a growing microtubule encounters a pre-existing microtubule. Depending on the value of collision angle, three different possible events were observed after collision (Dixit and Cyr, 2004): For shallow angles $\left(\lesssim 40^{\circ}\right)$, a growing microtubule bends toward the direction of the microtubule encountered and this kind of adaptive event is called zippering. For steep angles $\left(\gtrsim 40^{\circ}\right)$, a growing microtubule slips over the one encountered, called crossover event. Alternatively, the encounter leads to a catastrophe, where the initially growing microtubule switches to a shrinking state. A collision induced catastrophe is called induced catastrophe. During the course of zippering, two or more microtubules can form a bundle via the microtubule associated protein Microtubule Associated Protein (MAP65), which gives an enhanced microtubule stabilization within a bundle. Growing microtubules on the cell cortex often encounter domains of high curvature, particularly at cell edges or corners. Experimental observation on elongating root cells has revealed that a cortical microtubule encountering a sharp cell edge suffers more edge catastrophes than those encountering a semi-rounded edge (Ambrose and Wasteneys, 2008). In Fig. 1.5, we present a schematic diagram of these different collision induced events. Shrinking of a microtubule due to the retreating plus end (consequence of catastrophe) or treadmilling minus end may result in disappearance of the microtubule.

\section{From microtubule array to cell division}

In vivo imaging of cortical microtubules has revealed that microtubules nucleate at the cortex and gradually develop from an initially disorganized state into a final ordered array, over a time period of an hour (Wasteneys and Williamson, 1989; Kumagai et al., 2001; Shaw et al., 2003; Vos et al., 2003; Ehrhardt and Shaw, 2006). The ordered array that comes out as an emergent property of microtubule dynamics and interactions is relatively sparse, which eventually converges to a narrow circular bundle of microtubules. This narrow band of microtubules along with actin filaments and associated proteins, form the preprophase band (PPB) (Mineyuki, 1999; 


\section{Central dogma of plant microtubule dynamics}

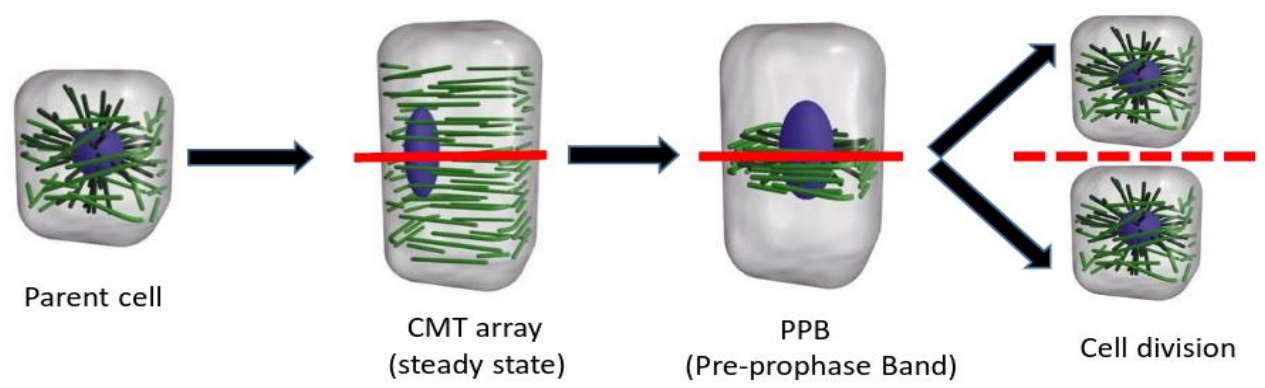

Fig. 1.6. A schematic description of the central dogma of plant microtubule dynamics. In the newly born daughter cell (or the youngest parent cell) cortical microtubules are shorter in length and located at the dispersed site of the cell cortex, with random local orientations. As the cell grows and gain maturity, the initially disorganised state of the microtubules develops into a well organised oriented array (CMT array). Later the relatively sparse array of microtubule aggregates to a dense ring, called preprophase band (PPB). PPB leaves a spatial marker for the insertion of the new division plane that guides the cell division. This way, the orientation of the microtubule array determines the orientation of the division plane (horizontal line, red color). Post division, the entire process of microtubule array dynamics is repeated in the daughter cells to trigger the next generation of cell division. The image is adapted from Wasteneys (2002).

Cleary et al., 1992; Granger and Cyr, 2000; McCurdy et al., 2001). Therefore the orientation of the PPB is same as the orientation of the initial sparse array of microtubules. In plant cells, during cell division the insertion site of the cell wall is predicted by the PPB, which is a unique plant cytoskeletal structure that creates a spatial mark for the insertion site of cell wall on the plant cell cortex. Several possible mechanisms were suggested to explain the formation of the PPB (Dhonukshe and Gadella, 2003; Hush et al., 1994; Vos et al., 2003). However, none of these suggested mechanisms provided a clear understanding of the physical mechanism behind the formation of the PPB. Based on experimental observation in tobacco Bright Yellow-2 (BY-2) suspension culture cells, Vos et al. (2004) proposed a "search and capture" mechanism of the PPB formation (see Fig. 1.7). In this study, the rate of dynamic instability of the cortical microtubules were observed to be increased outside the domain of PPB without shortening the microtubule length. At the same time microtubules were observed to be preferentially stabilized by the microtubule cross-linking activity of the developing PPB. This enables the microtubules to stochastically "search" for the domain of PPB in the cell cortex and get "captured" upon its encounter. For the initiation of the capture mechanism, a small offset of few cross-linked microtubules in the PPB forming domain was proposed to be sufficient to initiate a self-perpetuating process (Stoppin et al., 1994). In this experimental work, no evidence was found for the transportation of microtubules towards the PPB forming domain via active motor proteins (Asada and Collings, 1997; Reddy, 2001) or enhanced catastrophe of microtubules outside the PPB domain (Dhonukshe and Gadella, 2003). Experimental evidence of the stabilization of microtubules in the PPB domain that can catalyse 


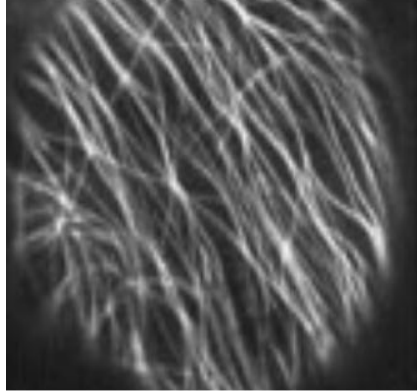

\section{CMT array}

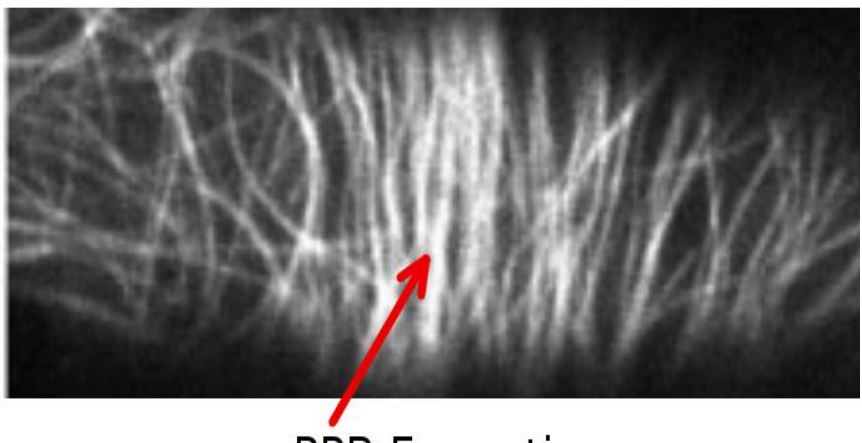

PPB Formation

Fig. 1.7. Experimental observation of the formation of preprophase band (PPB) in tobacco Bright Yellow-2 (By-2) suspension cells. The images are adapted from Vos et al. (2004).

the "capture" mechanism, was reported in Hush et al. (1994). The PPB plays a key role in determining the exact location (determining factor of symmetric/asymmetric division) of the division plane. During spindle formation and nuclear membrane breakdown, the PPB is also broken down, leaving behind a spatial marker for the correct insertion of the cell plate (Lloyd and Buschmann, 2007; Wright et al., 2009; Cleary, 2001; Dixit and Cyr, 2002; Mineyuki, 1999; Molchan et al., 2002; Pickett-Heaps et al., 1999; Valster et al. 1997). Cell divides through the formation of an outward growing cell plate that fuses with the parental plasma membrane and cell wall along the spatial signature left by the PPB (Staehelin and Hepler, 1996). We call this entire process of cortical microtubule directed plant cell division The central dogma of plant microtubule dynamics (see Fig. 1.6). Regarding the positioning of the PPB (hence the division plane), experimental evidence indicated a strong correlation with the corresponding nuclear position (Kimata et al., 2016). However, neither the actual determining factor of the location of the PPB nor the orientation mechanism of the initial sparse array of microtubule are currently understood.

\section{Genetic control of cell division orientation}

The molecular means of cell division patterning is the formation of an ordered array of microtubules. A part of the microtubule order may arise from intrinsic properties of microtubules and cell shape/geometry. The observed cell division patterns during plant morphogenesis are also developmentally controlled.In evolutionary sense, the genetic controls must have the potential to specify division patterns by overwriting the intrinsic (default) rules dictated by the "excitable system" of microtubule interactions on a specific cell cortex, when appropriate. Therefore, a fraction of the microtubule order, which essentially characterizes the type of plant morphogenesis, should come from the explicit genetic controls. As genetic regulators, the plant 
hormone auxin and a few transcription factors, among which the AUXIN RESPONSE FACTOR (ARF) and PLETHORA (PLT) proteins are known to be involved in the process of oriented cell division, thus likely to have influence on the microtubule order and array orientation.

\section{Auxin and MONOPTEROS/BODENLOS proteins}

In higher plants, Indole-3-acetic acid (IAA), the main biologically active auxin, has profound effect on plant growth and development.The ARF transcriptional regulator family is involved in controlling the expression of auxin response genes (Guilfoyle and Hagen, 2007; Boer et al., 2014; Li et al., 2016).ARFs are transcription factors that function by binding to auxin response elements (AuxREs) on the promoters of auxin response genes, activating or repressing the auxin response genes. ARF function is regulated by a second family of transcriptional regulators, the Aux/IAA repressors (Dharmasiri et al., 2005; Kepinski and Leyser, 2005). The later confer an auxin response to the ARF target genes. Examples of IAA and ARF proteins involved in cell division plane orientation are BODENLOS (BDL Hamann et al. (2002)) and MONOPTEROS (MP, Hardtke and Berleth (1998)). A stabilizing mutation in the $B D L$ gene, i.e $b d l$ mutation, deviates from normal development of Arabidopsis embryo, as early as the two-cell stage, at which the apical daughter cell of the zygote divides horizontally instead vertically (Hamann et al., 1999). A similar phenotype has been reported for mutations in the $M P$ gene, i.e. $m p$ mutation. Using $R P S 5 A$ as an embryonic expressing promoter driving $b d l$, misexpression of a nondegradable version of $b d l(R P S 5 A>>b d l)$ showed strong auxin insensitive phenotypes during later stages. During the $8 \rightarrow 16$-cell stage transition, nearly all cells divided abnormally (Yoshida et al., 2014), and the root and cotyledon initiation or the suspensor derived embryogenesis was lost (Rademacher et al., 2012). These experimental studies suggested a role for auxin in controlling oriented cell divisions during Arabidopsis embryogenesis.

\section{PLETHORA (PLT)}

The PLT1 and PLT2 proteins are essential for defining the root stem cell niche, i.e. maintaining the controlled cell divisions, cell growth and division plane orientations (Aida et al., 2004). Other transcription factors known to be involved in division plane orientation in Arabidopsis root are TARGET OF MP (TMO5) and its basic helix-loop-helix interactor LONESOME HIGHWAY (LHW). Though the role of TMO5 and LHW in altering the division plane orientation has been specifically studied in the context of the establishment of the vascular tissue, the exact role of PLT genes in division plane orientation is not yet explored (Rybel et al., 2013). plt1;plt2 mutants result in stem cell loss, loss of transit-amplifying cells and reduced 
cell expansion. In the root, the PLT proteins are expressed in gradients, where the expression level is highest in the meristematic zone and gradually decreases towards the elongation and differentiation zones (Galinha et al. 2007; Mähönen et al., 2014). In the meristematic zone, where the PLT level is high, the cells divide more frequently and display slow cytoplasmic growth. Whereas most epidermis-lateral root cap cells or cortex-endodermis cells divide anticlinally, their corresponding initial cells, which are close to the root quiescent center (stem-cell zone) of the root meristem, divide periclinally and grow at a slower pace. High levels of PLT in the stem-cell zone and distinct nature of cell division, cell growth and division plane orientation in this zone, suggests a significant role of these transcription factors in controlling cellular processes like cell growth and cell division. Consistently, the activated target genes of the PLT transcription factors are enriched for genes involved in cell division and growth (Santuari et al., 2016). It has been shown that the expression of the PLT transcription factors strongly correlates with a transcriptional response maximum to the plant hormone auxin in the root tip (Aida et al., 2004; Xu et al., 2006). Also, the PLT transcription factors have the ability to regulate the local biosynthesis of auxin (Pinon et al., 2013). Considering the role of auxin in controlling oriented cell divisions, PLT proteins may also be involved in division plane orientation.

\section{Modelling oriented microtubule arrays}

The PPB is formed through aggregation of an oriented sparse array of microtubules into a dense ring. Therefore, a key question towards the understanding of oriented cell division is to understand the formation of the orientated sparse array of microtubule from an initially disorganized state. Formation of the ordered array involves interactions of large numbers of spatially distributed microtubules. While each microtubule undergoes intrinsic stochastic dynamics, it encounters other microtubules through collision interactions, that may lead to self-organisation. Here, we can think of the cortical microtubules as the initial structure molecules (cellular components), which serve the role of excitable media for a cellular state. Different states of self-organisation of the cortical microtubules will drive the corresponding cell into different states of the microtubule array orientation (building blocks for morphological pattern formation), thus instructing division plane orientation. The complexity of the self-organisation process of the microtubules creates a burden to disentangle the individual contribution of the different microtubule dynamics parameters towards the final order and orientation of the microtubules array. Several attempts were made to visualize cortical microtubules in the plant cell cortex using different types of microtubule markers, where the average behaviour of the microtubules 
was reported as growing stiff polymers with dynamics instability properties at their ends. The dynamic instability parameters (growing speed, shrinking speed, catastrophe rates etc.) were quantified (Shaw et al., 2003; Chan et al., 2003; Dhonukshe and Gadella, 2003; Vos et al., 2004) by marking the microtubule tips through tagged microtubule-end associated proteins (Matov et al., 2010). A certain degree of variability was observed in the measured parameter values between the different studies. The probability of edge-catastrophe has been measured on elongating root cells (Ambrose and Wasteneys, 2008). Involvement of a large number of parameters and the stochastic nature of the individual microtubule dynamics make the experimental understanding of the self-organisation process of cortical microtubules difficult, if not impossible. However, the existing experimental studies inform us about the major ingredients (key parameters) that are involved in the array formation. The formation of the cortical microtubule array is a macroscopic phenomenon that depends on the average behaviour of many microscopic particles, i.e microtubules and their interactions. Mathematical/computational modelling has the ability to explain the average behaviour behaviour of a system of particles knowing only a few key characteristic parameters. Several computational modelling approaches have been proposed, which were mainly limited to the two dimensional plane (Zumdieck et al., 2005; Baulin et al., 2007; Shi and Ma, 2010; Dixit and Cyr, 2004; Allard et al., 2010; Tindemans et al., 2010). Some extensions to three dimensional surfaces were made, but application of those models was constrained to regular shapes such as cube (Ambrose et al. 2011) and cylinder (Tindemans et al., 2014). Studying the formation of ordered array of microtubules on realistic cell shapes demands a more generic modelling framework that can be used to simulate microtubule dynamics on any shape.

\section{From biological desiderata to modelling strategy}

Understanding microtubule dynamics on realistic cell shapes requires a novel modelling framework. The modelling strategy must be able to meaningfully represent experimental facts about the dynamics of cortical microtubules. Though experimental observation showed presence of microtubule nucleation from existing microtubules, for simplicity we ignore such nucleation and consider only the individual nucleation of microtubules, i.e. homogeneous (nucleating at random locations of cell cortex) and isotropic (random directions of growth upon nucleation). An ordered array of microtubules is formed from an initially disorganized state, and it can be anticipated that the interactions among microtubules have a significant role in the formation of an ordered array. Accordingly, inclusion of microtubule interactions (mainly collision events: zippering, induced-catastrophe and 
crossover) to the modelling framework is essential. When microtubules grow in a smooth region of the plant cell cortex, the persistence length of a microtubule is much bigger than the average length, allowing to model the individual microtubule as a stiff rod-like particle. However, in order to mimic experimentally observed treadmilling motion, we must enable dynamic instability at the growing end and a small shrinking velocity at the minus end of the approximated microtubules. Attachment of microtubules to the cell cortex automatically implies an influence of cell geometry on microtubule interaction frequencies. Cell size will have a direct impact on microtubule interactions, as microtubules on smaller cells will experience higher frequencies of induced catastrophes. Microtubules that encounter a cell-edge will suffer edge-catastrophe in a probabilistic way that depends on the curvature of the cell-edge. As the curvature of the cell-edge is one of the topological properties of a given shape, here again cell geometry will influence microtubule dynamics and organisation. Accordingly, the modelling platform needs to include the effect of cell-edge catastrophe in microtubule dynamics. The intrinsic difference in cell membrane (the inner membrane cortex) properties between two cell faces that have different developmental-ages, requires the modelling framework to treat each of the cell faces differently. In this way, intrinsic cell membrane (cortex) properties can be translated into different degrees of microtubule stability. Finally, the confinement of the dynamics of cortical microtubules to the surface (cortex) of plant cell effectively reduces the problem from three to two dimensions. We can exploit this advantage by treating the cell shape as two dimensional surface but connected in three dimensions in a topologically correct way.

\section{Research outline}

Plant morphogenesis is controlled by oriented cell division and controlled cell expansion, and both of these two processes are influenced by the oriented array of cortical microtubules. In this thesis work, we aimed to understand the formation of the oriented array of microtubules by simulating microtubule dynamics on realistic plant cell shapes (cortex), taken from Arabidopsis early embryos and the root epidermal cell layer. Through these studies, we sought to explain the experimentally observed cell division pattern in the context of almost non-growing embryo stages and in the anisotropically growing root epidermis.

In chapter 2, we describe the implementation of microtubule simulations through a novel modelling framework, that allowed us to study microtubule dynamics on the surface of arbitrary shapes. In chapter 3, we describe methods to parametrize the simulations before applying the modelling approach directly into the biological problems.

In chapter 4, we explore the generic role of microtubule interactions and 
cell shapes in the formation order and orientation of the microtubule array, for convenient biological interpretation of the simulation results.

In chapter 5, using a combination of experiments and simulations, we investigated the oriented cell division pattern during Arabidopsis early embryogenesis. We mainly focused on the first five division cycles of the zygote. Two cell division patterns were addressed, first the normal wild type and second, the division pattern after BDL mutation.

Chapter 6 addresses the role of PLT proteins in division plane orientation of the root. Through a combination of experiment and simulation, we studied division plane orientation in epidermal cells and their initials, both in wild type and after PLT induction.

In Chapter 7, the research from the experimental chapters is linked together and placed in the context of the developmental biology of plant morphogenesis. We end with recommendations for future research. 


\section{Chapter 2}

\section{Implementation of simulations}

The plant microtubule cytoskeleton is composed of a large number of spatially extended particles, each endowed with its own intrinsic stochastic dynamics, and is capable of non-equilibrium self-organization through collisions between these particles. Modelling the complex process of selforganisation of microtubules is challenging. So far, various authors modelled the dynamics and self-organisation of cortical microtubules. In one such model, microtubules were described as a rigid rod and simulated on a square domain where microtubule dynamics were subjected to periodic boundary conditions at the domain edges (Baulin et al., 2007). In this model, average speeds of growth and shrinkage were considered as an approximation of the fluctuations in growth speed due to dynamic instabilities at the microtubule tips. For microtubule interactions, when the plus-end of a microtubule encountered another microtubule, it was instructed to stop and resume its growth only upon removal of the block. Microtubules were nucleated homogeneously and isotropically and discrete time steps were used for dynamic evolution of microtubules. A similar model was prescribed by Shi and Ma (2010), but with reflecting boundary conditions at the domain edges. Also, fluctuations in growth/shrinkage speed due to dynamic instability of the growing tips, were explicitly considered. However, all these models did not implement experimentally observed collision rules ${ }^{(1)}$. Based on these collision rules, an iterative Monte Carlo modelling technique was implemented by Dixit and Cyr (2004), where each iteration was represented as a finite time step. The simulation was conducted on a planar domain where microtubules that encountered the edge of the domain were allowed to behave stochastically. A further enriched simulation approach was taken by Allard et al. (2010), where microtubules were simulated on a square domain with periodic boundary conditions at the domain edges. A Gillespie algorithm (Gillespie, 1977), was implemented to describe the dynamic instability at microtubule plus ends that resulted in a variable time step. However, a fixed time step was used to detect and resolve microtubule collisions. In this model, for the first time, the consequences of edge-catastrophe were explored by incorporating edge-induced catastrophe on microtubule dynamics at a pair of opposite edges of the simulation domain. In an extended version of this model, dynamics of microtubules were simulated on a cubic shape (Ambrose et al., 2011), where the faces of the cube were approximated

${ }^{(1)}$ collision rules: zippering, induced-catastrophe, crossover 
by square domains. All the proposed models mentioned so far used finite time step simulation. As a result, most of these simulations were slow and inaccurate $^{(2)}$. To overcome these limitations, an intrinsically exact and faster simulation technique was introduced (Tindemans et al., 2010, 2014). In this approach an event-driven methodology was adopted that encompasses both spontaneous stochastic changes in microtubule state as well as deterministic collisions. Instead of accounting for all of the different microtubule dynamics parameters separately, this modelling framework used a single input control parameter as identified by Hawkins et al. (2010). The use of this control parameter assured, in advance, the formation of an ordered array of microtubules within a pre-defined value of simulation end time. From the existing models that simulate cortical microtubules, most of the modelling frameworks simulate microtubules on a two dimensional domain. Only in three of the existing frameworks microtubules were simulated on a three dimensional surface, but limited to cubic (Ambrose et al., 2011) and cylindrical surfaces (Eren et al., 2010; Tindemans et al., 2014).

We aimed to simulate microtubule dynamics on shapes realistically reflecting the cortex of specific plant cells. As plant cells come in a variety of sizes and shapes, there is no unique way to describe them mathematically. Therefore, none of the existing modelling frameworks can be used for microtubule simulation. It is however possible to approximate a given cell shape (cortex) by a triangulated surface mesh, where each triangle represents a part of the whole cell cortex. The microtubule dynamics can be computed on each of these triangles with proper boundary conditions that propagate the dynamics to neighbouring triangles, thus allowing us to study the microtubule self-organisation on arbitrary cell shapes/geometries. In this chapter, we describe a framework to study the dynamics of microtubules on triangulated approximations of arbitrary two dimensional or three dimensional surface. In Sec. 2.1, we describe the processing of the confocal image of plant cells for simulation. i.e transforming confocal images into a triangulated surface mesh. In Sec. 2.2, we explain the techniques behind simulating microtubules on a three dimensional surface mesh. Finally, in Sec. 2.3, we prescribe an extension of the modelling framework from a three dimensional surface mesh to a two dimensional (plane) mesh.

\subsection{Processing confocal images for simulation}

Using the image processing software morphographX (de Reuille et al., 2015), we segmented experimentally obtained confocal images (Fig. 2.1) and extracted all the different cell shapes. Then, cell shapes were approximated

\footnotetext{
${ }^{(2)}$ Inaccurate as finite time step made the accuracy of simulation output to be depended on the choice of time interval
} 


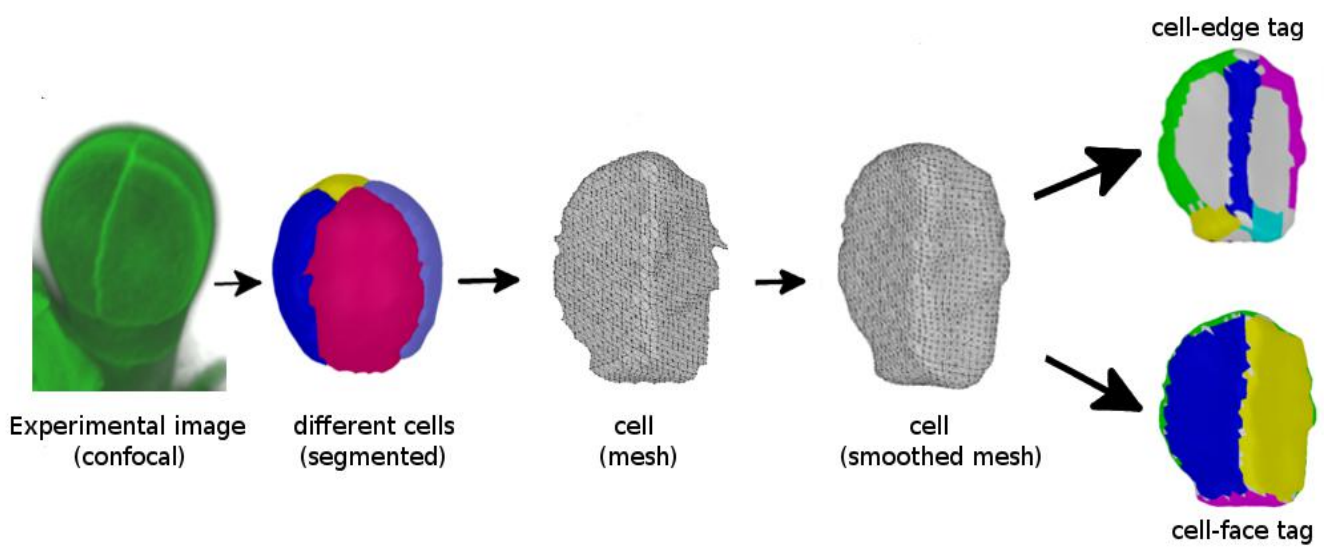

Fig. 2.1. Image processing steps in transforming confocal image of cell into triangulated surface mesh, to be used as simulation input. Experimentally obtained confocal image is segmented to extract different cell shapes, the cell shapes are approximated by triangulated surface, unacceptable artefacts are removed by using appropriate smoothing filter, and different cell-faces and cell-edges are tagged uniquely. The output is stored in a simulation input mesh file.

with a triangulated surface mesh. Due to unavoidable noise in experimentally acquired images, the triangulated approximation of a cell shape often contained some artefacts. We used the surface mesh processing software meshLab (Cignoni et al., 2008) to fix such artefacts. The same software was used to tag the different cell-edges and cell-faces by appropriately colouring the associated triangles. We calculated the average value of a cell edge-angle $\theta_{E d g}^{a v g}$ (see Appendix A.1, Measure of edge-angle), associated with each edge, by using a custom made computer program written in $\mathrm{C}^{++}$. This program is designed to attribute a cell-edge with a unique cell-edge tag. All the triangles belonging to that cell-edge copy the associated cell-edge tag. Similarly, a cell-face gets a unique cell-face tag. All the triangles belonging to that cell-face copy the associated cell-face tag. Finally, all this information is stored in a single mesh file, where each triangle has a cell-edge tag and a cell-face tag.

\subsection{Description of the simulation technique}

We designed the simulation (modelling) framework to accept the triangulated approximation of a given shape as input mesh file. In the input mesh file the surface information is encoded via attributing each triangle with: an edge-tag along with the value of the associated edge-angle, and a face-tag (see Fig. 2.2). The simulation output allowed us to track and visualize the dynamics of microtubules at any time point of simulation. In the following sections, we describe the details of the simulation technique. In Sec. 2.2.1, we transform the 3D-triangulated surface into a set of 2D-triangles. In Sec. 2.2.2, we describe the dynamics of microtubules on individual triangles. Propagation of microtubules from one triangle to an 


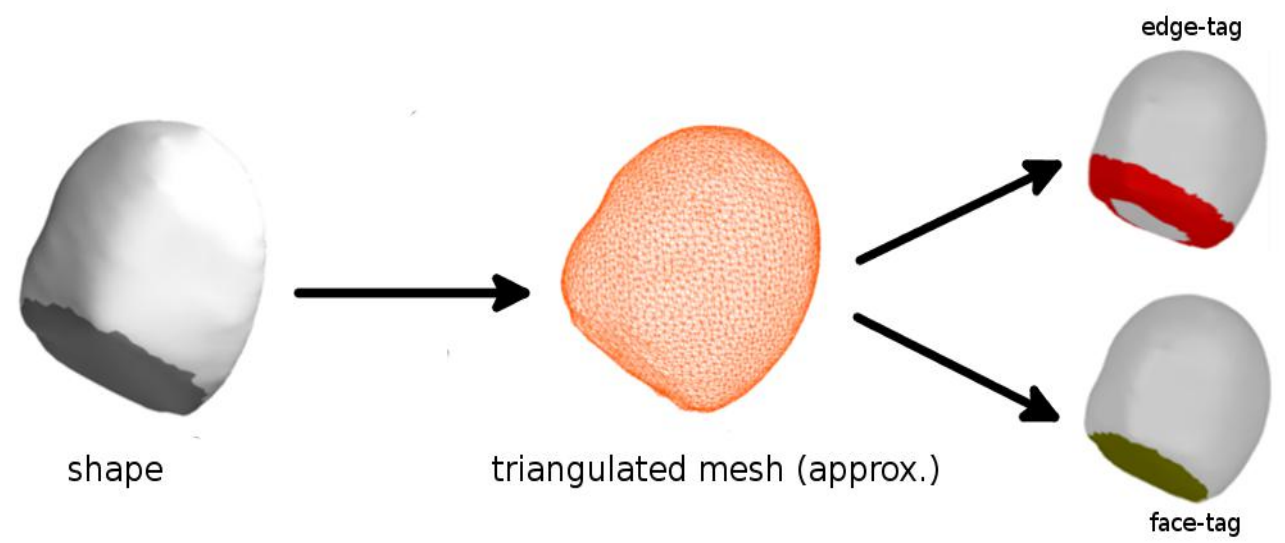

Fig. 2.2. A given shape is approximated by a triangulated surface. Using our custom made C++ program, we assign a unique tag for each of the different faces and edges. We store the output in a mesh file, where all triangles associated to a specific face or edge copy the respective face/edge tag.

adjacent triangle is described in Sec. 2.2.3. In Sec. 2.2.4, we explain the procedure of re-constructing the three dimensional surface and mapping microtubule segments from the set of two dimensional triangles to the three dimensional surface.

\subsubsection{D-triangulated surface to $2 \mathrm{D}$-triangles}

In the triangulated approximation of the surface, a microtubule, being a growing/shrinking polymer, can move from one triangle to another. In order to propagate microtubule dynamics between the triangles, we developed an algorithm to establish a connectivity graph between two adjacent triangles (Ueng and Sikorski, 1996). This graph helped to uniquely orient the triangulated surface, i.e the normals of all the triangles collectively orient either inward or outward to the surface. We transformed the 3D-triangulated surface from three dimensional $x-y-z$ space to a set of 2D-triangles on the two dimensional $x-y$ plane. We performed this transformation in two steps: (1) In three dimensions, rotate all the triangles (see Appendix A.2, Quaternion rotation of triangles) to re-orient their normals parallel to the positive $z$-axis direction, and (2) from this new orientational configuration in three dimension, translate all the triangles along the $z$-axis and place them on the two dimensional $x-y$ plane. Here, making the surface oriented gave another advantage of having the normals of all the rotated triangles, collectively parallel to the positive $z$-axis direction. This way the orientation of the inner or outer side of the three dimensional surface was preserved, even though the surface was split into pieces of two dimensional triangles. The unique orientation of the surface was crucial to propagate microtubule dynamics consistently either over the inner side or the outer side of the surface. Unless positioned on the same plane in three dimensions, the adjacent triangles attached to each other through a shared edge in three dimension, 


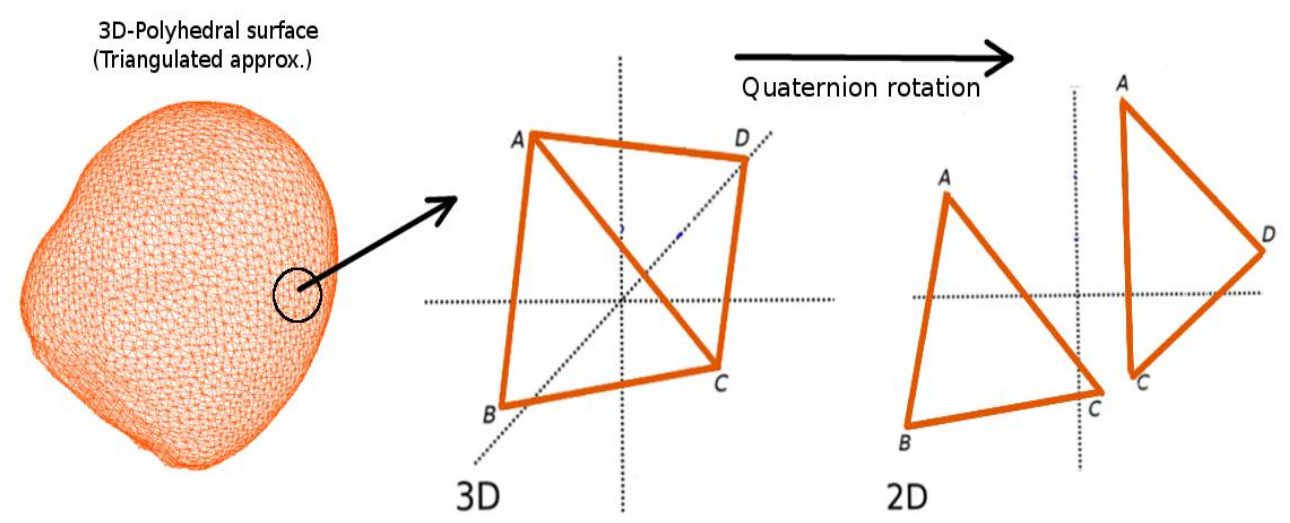

Fig. 2.3. Quaternion rotation of triangles from three dimensional surface mesh to two dimensional plane. $\triangle A B C$ and $\triangle A D C$ are connected through a shared edge $A C$ in three dimensional $x-y-z$ space and are separated away after quaternion mapping to two dimensional $x-y$ plane.

get separated after their quaternion rotation into two dimensions (see Fig. 2.3). As a result, a closed triangulated surface in three dimensions was transformed into a scattered set of triangles in two dimensions.

\subsubsection{Microtubule dynamics on individual triangles}

We captured the local microtubule dynamics by studying their motion and interactions in each individual triangle. In order to nucleate microtubules on the surface, first we randomly selected a triangle from the entire set and generated a random point $P(x, y)$, uniformly from within the selected triangle (see Appendix A.2, Homogeneously distributed points within a triangle). This reflected our approach of considering homogeneous nucleation of microtubules. Upon selecting the position of the nucleation point, we initiated microtubule growth by assigning a random angle of nucleation $\theta_{n c l}$, chosen uniformly from the range $[0,2 \pi]$ (see Fig. 2.4 , left panel, MT nucleation). This corresponded to our consideration of isotropic nucleation of microtubules. To deal with microtubule collision events, we implemented an event-driven simulation technique as described in Tindemans et al. (2014). This approach doesn't introduce intrinsic uncertainties in the time domain, and is both accurate and fast. At any time, we determined the time of the next event and fast-forwarded the system state to that time, updated the microtubule lengths to reflect that time, and immediately processed the event. We repeated this cycle until the system reached to the predetermined end time of simulation. In our simulation, we separated all the events into two categories: stochastic events and deterministic events.

\section{Stochastic events}

The stochastic events associated with the microtubules are independent and result from random processes described by nucleation rate $\left(r_{n}\right)$, spontaneous 


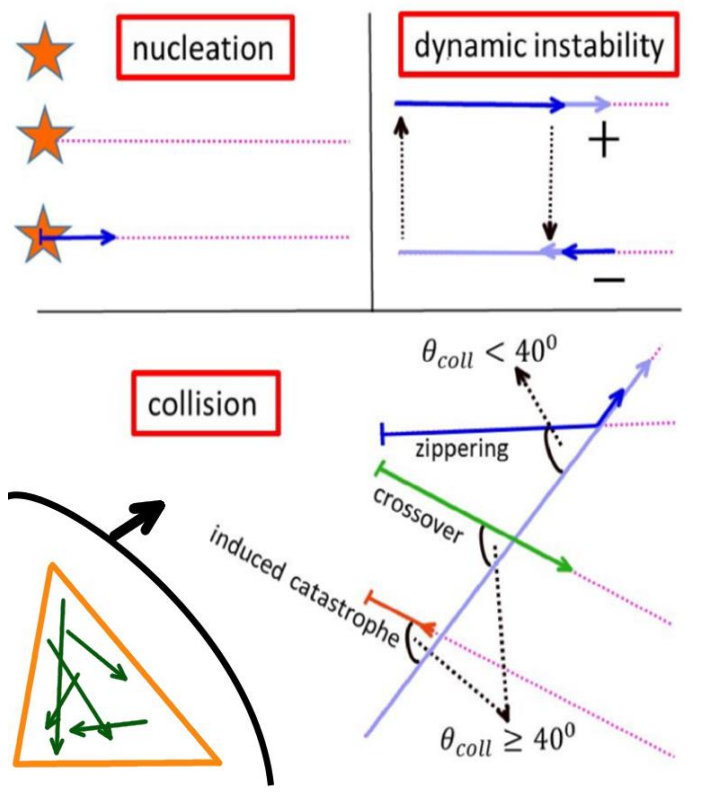

MT events

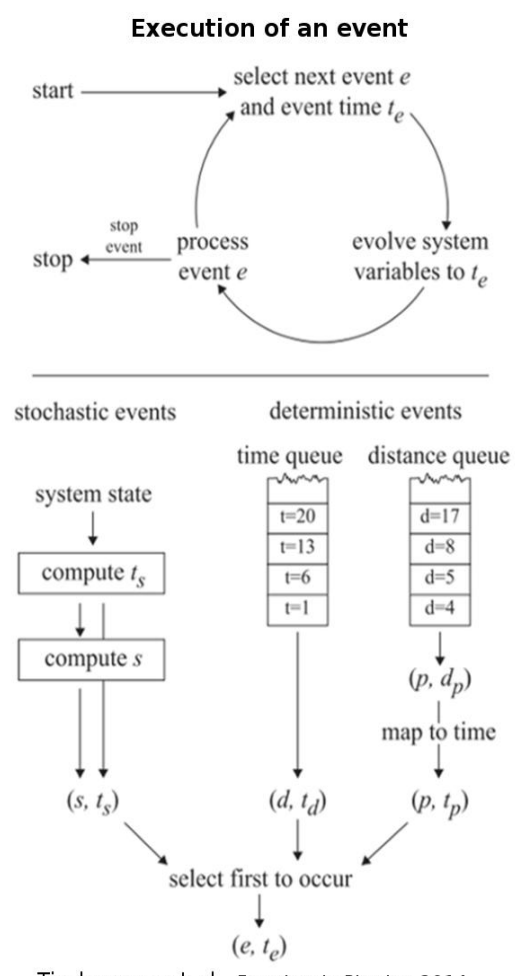

Tindemans et al. Frontiers in Physics, 2014 Fig. 2.4. Simulation description of microtubule events on an individual triangle. Microtubules are nu-
cleated homogeneously and isotropically, upon nucleation a straight line trajectory is assigned as a track cleated homogeneously and isotropically, upon nucleation a straight line trajectory is assigned as a track
for growth. Dynamic instabilities of microtubule tips are included through specification of spontaneous catastrophe and rescue rates. Type of collision event, i.e. zippering/induced-catastrophe/crossover, is determined on basis of the intersection angle between microtubule trajectories (left panel). Selection process of an event $(e)$ to occur at a time $\left(t_{e}\right)$ is schematically shown (right panel), where subscript 's' stands for stochastic events (nucleation, catastrophe and rescue) and the subscript 'd' stands for deterministic events (collisions, disappearance, edge-catastrophe and simulation control).

catastrophe rate $\left(r_{c}\right)$ and rescue rate $\left(r_{r}\right)$. In our simulation, we choose all the event rates to be constant between successive events. Following the description of Tindemans et al. (2014), we calculated the time interval $t_{\text {stoch }}$ to the next stochastic event as,

$$
\Delta t_{\text {stoch }}=-\frac{\log u}{R}
$$

where $u$ is a uniform random variate on the interval $(0,1)$ and $R$ is the total rate of all the stochastic events and expressed as,

$$
R=r_{n} A+r_{c} N^{+}+r_{r} N^{-},
$$

where, $A$ is the area of the triangulated surface, $N^{+}$and $N^{-}$are the number of growing and shrinking microtubules, with speed $v^{+}$and $v^{-}$respectively. We used a kinetic Monte Carlo algorithm (Fichthorn and Weinberg, 1991) in determining the next stochastic event time. Memoryless property of stochastic events allowed us to recalculate the $\Delta t_{\text {stoch }}$ upon its invalidation, such as after the execution of any deterministic event. 


\section{Deterministic events}

The deterministic events associated with the microtubules include collisions, disappearance, edge-catastrophe and simulation control (i.e extracting simulation output at fixed time intervals). All these events are deterministic events as the event time is fully determined by the state of the system. To determine and execute the deterministic events, we followed the procedure described by Tindemans et al. (2014). Each time a microtubule was nucleated, it was assigned a unique trajectory. This helped to determine the location of the collision between two microtubules by calculating the intersection point of their trajectories (see Appendix A.2, Intersection point of two line segments). All the possible intersections between the trajectories of the microtubules were calculated and stored in a list, which was used for rapid evaluation of future collision events. In case of microtubule bundle formation, one trajectory was shared among all the members of the bundle. To enhance simulation speed, microtubule positions were updated only when an event has taken place or a simulation output was extracted. For an instantaneous state of the system, deterministic events occurred at a predicted time, allowing to pre-compute the required times of all the future deterministic events and store them in a queue. At any given time, the front of the queue indicated the time interval to the first deterministic event. In general, the occurrence of an event (both deterministic and stochastic types) changed the state of the system, resulting in new deterministic events to be added to the queue, or outdated some events to be invalidated. In our simulation, we used a finite tubulin pool, this resulted in the speed of a growing plus end, at any time $t$, to be dependent on the collective length $L(t)$ of all microtubules in the system,

$$
v^{+}(t)=v_{0}^{+}\left(1-\frac{L(t)}{L_{\max }}\right)
$$

where, $L_{\max }=\rho_{t u b} A$ is the length equivalent of the total amount of tubulin in the cell cortex of area $A$ and finite tubulin density $\rho_{t u b}$, and $v_{0}^{+}$is the growth speed under the assumption of infinite tubulin pool. As the speed of the growing plus end $v(t)$ varies in unpredictable ways, this made it impossible to pre-compute collision or boundary traversal times. For this situation, we created another deterministic event queue. Instead of storing (and sorting) events by their expected time of occurrence, this queue stored (and sorted) the distance that a growing plus end will have to travel to reach the point of collision. At any specific time, we meaningfully converted the distance corresponding to the first event to an event time, which we compared with the event time from the other deterministic queue and the stochastic event time. Based on this comparison, we determined the event that will occur first (see Fig. 2.4, right panel). If a collision event 


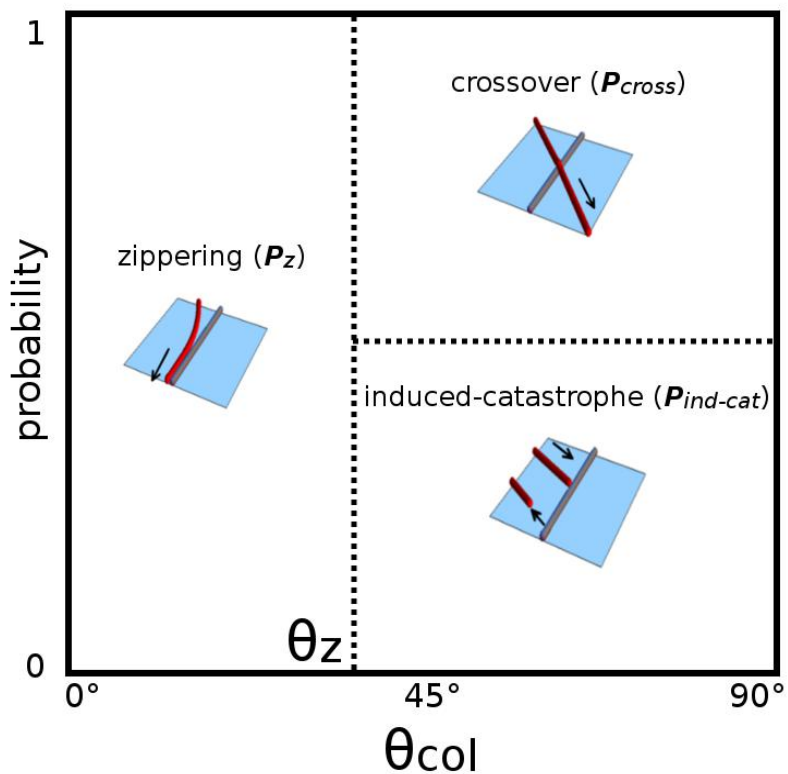

Fig. 2.5. Schematic representation of the probabilities of zippering, crossovers and inducedcatastrophes. This is an approximation of the observations by Dixit and Cyr (2004), combined data from MBD-DsRed and YFP-TUA6 labeling. For the angle of collision $\theta_{\text {col }} \leq 40^{0}$, namely zippering angle $\left(\theta_{z}\right)$, the probability of zippering was approximated to be $P_{z}=1$, i.e no crossover/inducedcatastrophe. For the angle of collision $\theta_{\text {col }}>40^{0}$, total probability was distributed equally to the

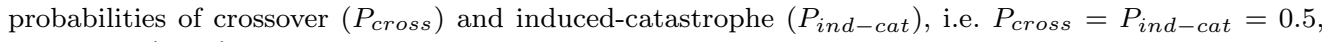
means no zippering.

was selected, based on the angle of collision $\left(\theta_{\text {col }}\right)$, a zippering/inducedcatastrophe/crossover was executed (see Fig. 2.5). The minus end of the microtubules was allowed to shrink with a constant treadmilling speed $v^{t m}$.

\subsubsection{Propagating microtubule dynamics}

Two adjacent triangles in the mesh were connected via the edge connectivity graph, a graph through which each edge of a three dimensional triangulated surface remembers the two associated triangle identities. This means for the set of two dimensional triangles, that each triangle edge has a unique link to an edge of another adjacent triangle. We used this edge-to-edge link to propagate microtubule dynamics from one triangle to another triangle. $\triangle A B C$ was connected with $\triangle A D C$ through the edge $A C$, referred to be a connectivity link. Transition of a microtubule from $\triangle A D C$ to $\triangle A B C$ through the edge $A C$ in the three dimensional surface implied, in two dimensional plane,

1. Translation of the associated growing microtubule tip from edge $A C_{(\triangle A D C)}$ of $\triangle A D C$ to edge $A C_{(\triangle A B C)}$ of $\triangle A B C$.

2. Rotation of the microtubule trajectory from $\triangle A D C$ to $\triangle A B C$.

Using an affine transformation map (see Appendix A.2, Affine transformation mapping) on the coordinates of the growing microtubule plus end tip 
at point $P^{\prime}\left(x^{\prime}, y^{\prime}\right)$ of edge $A C_{(\triangle A D C)}$, we translated (Fig. 2.6 left panel) the tip to the edge $A C_{(\triangle A B C)}$. Next, we rotated (Fig. 2.6 right panel) the
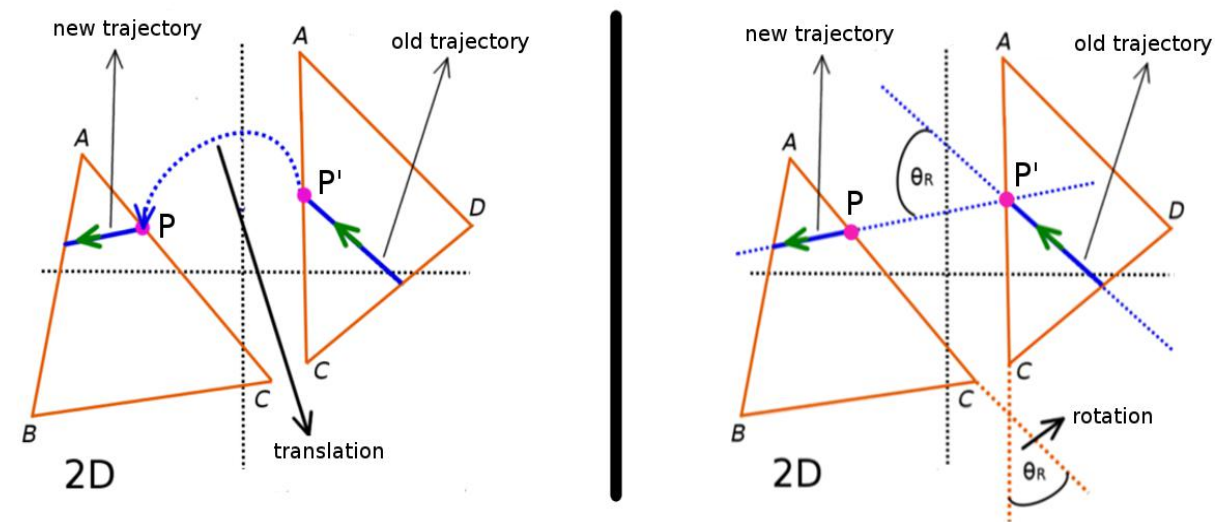

Fig. 2.6. Propagating microtubule dynamics from one triangle to another triangle. Affine transformation map is used to translate a growing microtubule tip from edge $A C_{(\triangle A D C)}$ of $\triangle A D C$ to edge $A C_{(\triangle A B C)}$ of $\triangle A B C$ (left panel). Upon translation, microtubule growth direction is rotated by an angle $\theta_{R}$ (see Eqn. 2.3 that equals the angle between $A C_{(\triangle A D C)}$ and $A C_{(\triangle A B C)}$. A new trajectory along this rotated direction is drawn for the microtubule to continue its dynamics on $\triangle A B C$ (right panel).

microtubule trajectory with rotation angle,

$$
\theta_{R}=\arccos \left(\frac{\overrightarrow{A C}_{(\triangle A B C)} \cdot \overrightarrow{A C}_{(\triangle A D C)}}{|\overrightarrow{A C}|^{2}}\right)
$$

This rotation in two dimensional plane was necessary to keep the microtubule growing along its original straight line path on the three dimensional surface.

\section{Edge-catastrophe}

For microtubules that encountered an edge of the triangulated surface, we implemented edge-catastrophe according to Ambrose et al. (2011). Due to the triangulated approximation, a cell edge was composed of many triangles. For each pair of such triangles $\left(T_{i}, T_{j}\right)$ neighbouring each other, we defined a unit vector $t_{i, j}$ along their communicating edges (a single shared edge of two triangles was split into two edges in two dimensions, but virtually linked through the connectivity graph). In the input mesh file, all the triangles were attributed with the average angle value $\theta_{E d g}^{a v g}$ of the cell-edge (Sec. 2.1) to which they belong. Having this information in hand, whenever a microtubule propagated from one triangle to another, based on the associated cell edge-tag, we implemented the probability of local edge-catastrophe $p_{e d g}^{(i, j)}$ at the crossing edge, by distributing the probability of total edge-catastrophe $P_{E d g}$ among each pair of triangles as illustrated in 

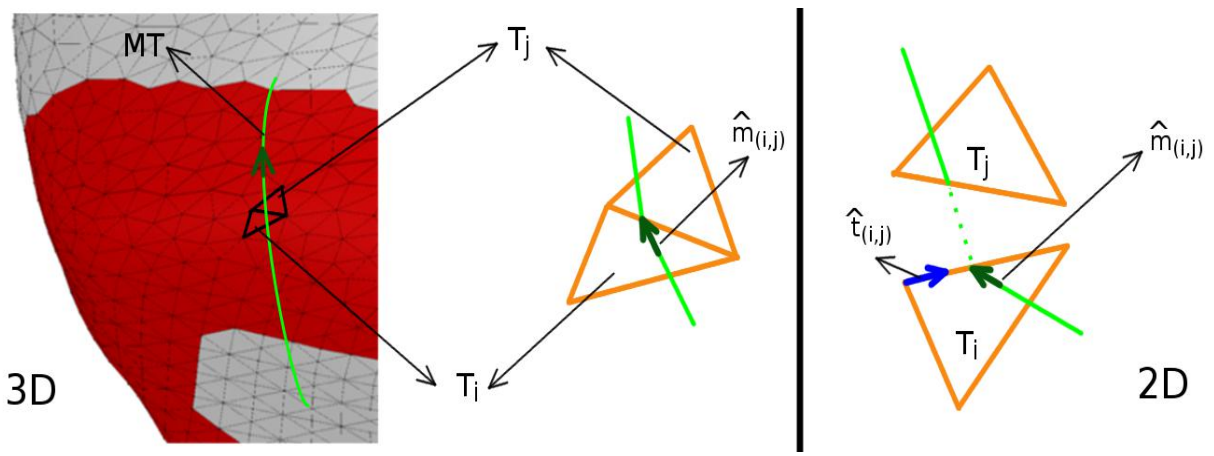

Fig. 2.7. Implementing edge-catastrophe in microtubule dynamics. When a microtubule passes through an edge of a triangulated surface, we implement the edge-catastrophe via local bending of microtubules through a set of triangle pairs $\left(T_{i}, T_{j}\right)$, which belongs to that edge (left panel). In the two dimensional triangle set, we use Eqn. 2.4 to execute local edge-catastrophe at the edge $\left.t_{(} i, j\right)$ of $T_{i}$, when a microtubule moves from $T_{i}$ to $T_{j}$ (right panel).

Fig. 2.7 with (see Appendix A.2, Local distribution of edge-catastrophes),

$$
p_{e d g}^{(i, j)}=\frac{E_{c a t} \cos ^{-1}\left(\hat{n}_{i} \cdot \hat{n}_{j}\right)}{2 \theta_{E d g}^{a v g}}\left(1-\cos \left(\cos ^{-1}\left(\hat{n}_{i} \cdot \hat{n}_{j}\right)\left(1-\left(\hat{m}_{(i, j)} \cdot \hat{t}_{(i, j)}\right)^{2}\right)\right)\right)
$$

where, $0 \leqslant E_{\text {cat }} \leqslant 2$ is an edge-catastrophe multiplier, specific to a certain edge and its value depends on the biomechanical properties of that edge. $\hat{n}_{i}$ and $\hat{n}_{j}$ are normals to $T_{i}$ and $T_{j}$ respectively, and both pointing either inwards or outwards to the triangulated surface mesh. $\hat{m}_{(i, j)}$ is the growth direction of a microtubule passing from $T_{i}$ to $T_{j}$.

\section{Face-stability}

For a triangle, a lower value of spontaneous catastrophe $\left(r_{c}\right)$ results in a higher average length of the associated microtubule segments, leading to a longer lifetime of the segments, in comparison to another triangle with higher value of $r_{c}$. This enhancement in lifetime can be interpreted
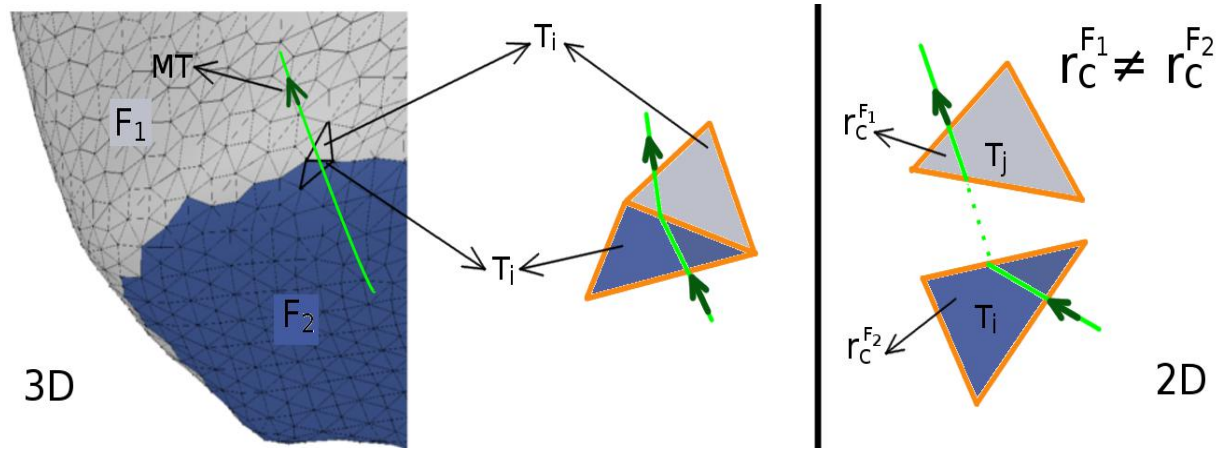

Fig. 2.8. Implementing face-stability in microtubule dynamics. On a triangulated surface, when a microtubule passes from a triangle $T_{i}$ of a face $F_{1}$ to a triangle $T_{j}$ of another face $F_{2}$, we modulate the face-stability via the local value of spontaneous catastrophe in these triangles (left panel). In the two dimensional triangle set, when a microtubule propagates from $T_{i}$ to $T_{j}$, we perform an update to the microtubule about a change in the rate of spontaneous catastrophe from $r_{c}^{F_{1}} \rightarrow r_{c}^{F_{2}}$. 
as endowing enhanced stability of the microtubule segments by the corresponding triangle. Therefore, we implemented the idea of difference in stability of microtubules at different faces of the triangulated surface, via face specific different rates of spontaneous catastrophes. We illustrate the implementation technique of microtubule stabilization in Fig. 2.8. All the triangles belonging to a face $F_{1}$ were assigned with a rate of spontaneous catastrophe $r_{c}^{F_{1}}$ and the triangles belonging to an adjacent face $F_{2}$ were assigned with a rate of spontaneous catastrophe $r_{c}^{F_{2}}$, where $r_{c}^{F_{1}} \neq r_{c}^{F_{2}}$. For transition of microtubules between the triangles of same face $\left(F_{1}\right.$ or $\left.F_{2}\right)$, in terms of the rate of spontaneous catastrophe, no changes were considered. However, when a microtubule propagated from a triangle $T_{i}$ of $F_{1}$ to a triangle $T_{j}$ of $F_{2}$, the change in the rate of spontaneous catastrophe from $r_{c}^{F_{1}} \rightarrow r_{c}^{F_{2}}$ was automatically updated.

\subsubsection{Mapping microtubule segments from 2D-plane to $3 \mathrm{D}$-polyhedral surface}

We applied inverse quaternion rotation (see Appendix A.2, Quaternion rotation of triangles) on the two dimensional triangles and the microtubule

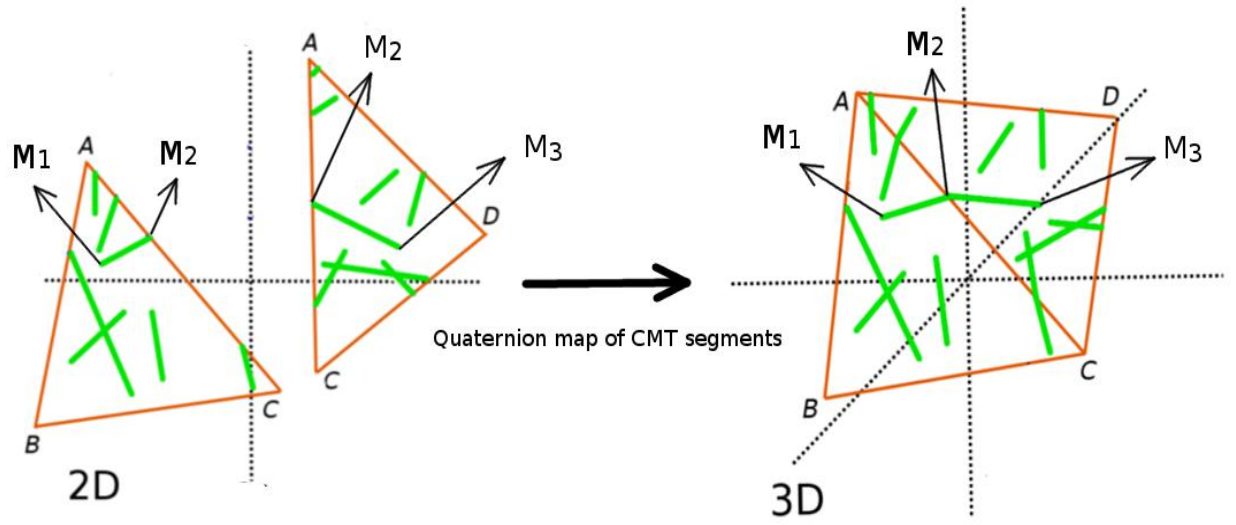

Fig. 2.9. Mapping microtubule segments from two dimensional plane to three dimension. To map a microtubule segment $\overline{M_{1} M_{2}}$ of $\triangle A B C$ from two dimensional plane to three dimension, we apply the inverse quaternion rotation operator of $\triangle A B C$ to the segment $\overline{M_{1} M_{2}}$. A similar operation can be performed to a microtubule segment $\overline{M_{2} M_{3}}$ of $\triangle A D C$. In their three dimensional position, if $\triangle A B C$ and $\triangle A D C$ are connected to each other via the edge $A C$, the microtubule segments $\overline{M_{1} M_{2}}$ and $\overline{M_{2} M_{3}}$ will also get connected.

segments present on the associated triangles, to reconstruct the three dimensional surface while containing the microtubule segments. Through this transformation, the segments of a microtubule which appeared disconnected in two dimensional triangles, got connected in the three dimensional surface. As illustrated in Fig. 2.9 , the segment $\overline{M_{1} M_{2}}$ was attached to $\triangle A B C$ and the segment $\overline{M_{2} M_{3}}$ was attached to $\triangle A D C$ in two dimensional plane. After the inverse mapping, these two segments got connected in the three 
dimensional surface and represented a single microtubule with end points $\left(M_{1}, M_{3}\right)$.

\section{An overview of the simulation approach}

The result of above set of operations described above is a program that allows to simulate microtubule dynamics on the triangulated surface of any shape. This means, we can simulate microtubule dynamics on the triangulated approximation of realistic plant cell surface. In Fig. 2.10, we
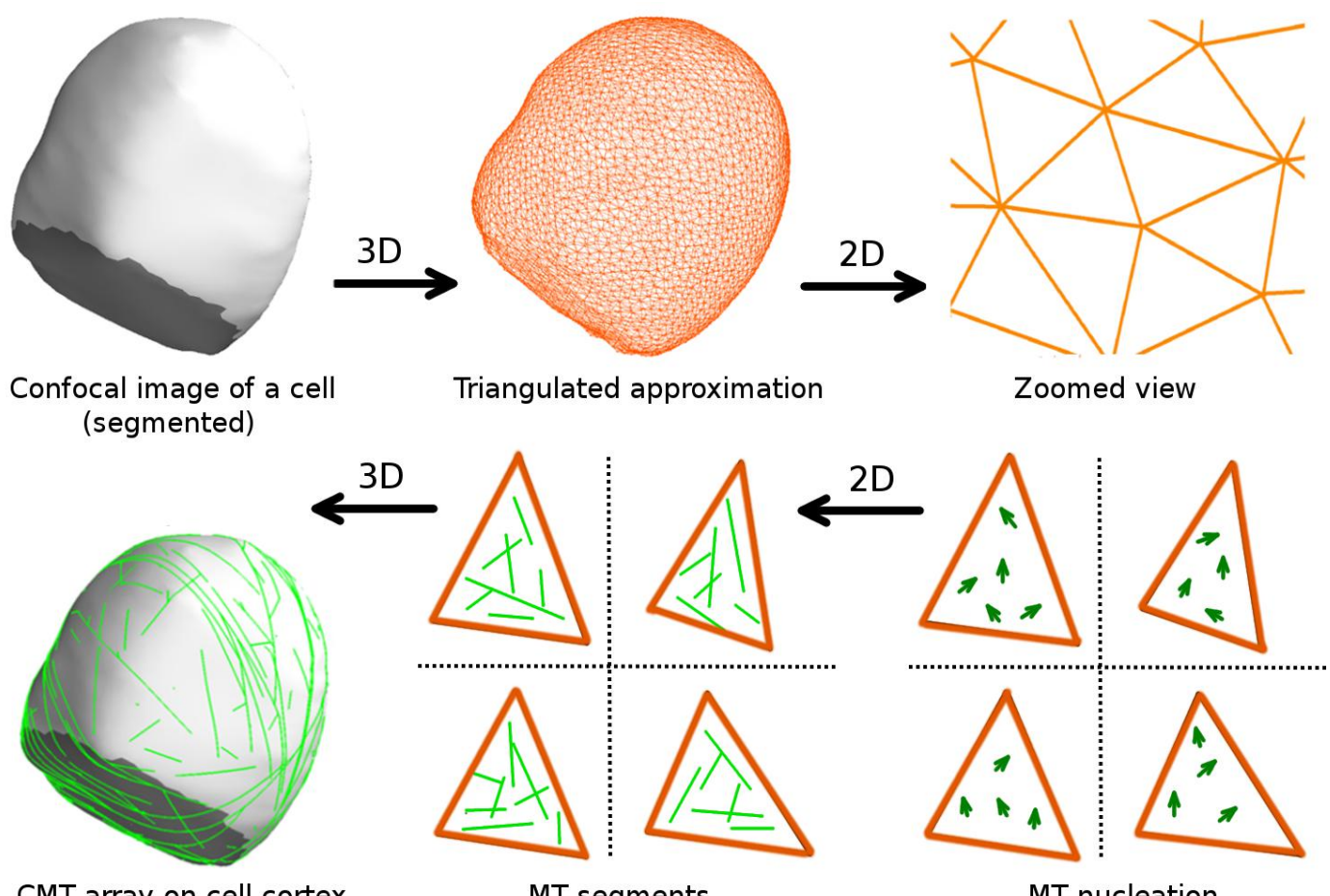

CMT array on cell cortex

MT segments

MT nucleation

Fig. 2.10. A schematic overview of our simulation approach.

present an overview of the entire simulation approach, which we coded in a computer program written in $\mathrm{C}^{++}$.

\subsection{Extension to two dimensional plane}

Our simulation framework allows us to study microtubule dynamics on triangulated three dimensional surfaces. To use this framework on a two dimensional plane, i.e. a two dimensional quadrilateral $\square A B C D$, we constructed a three dimensional surface by connecting all the vertices of $\square A B C D$ to an another point $O$, which was located outside the quadrilateral plane (see Fig. 2.11, virtual 3D-polyhedral). In order to meet the requirement of 

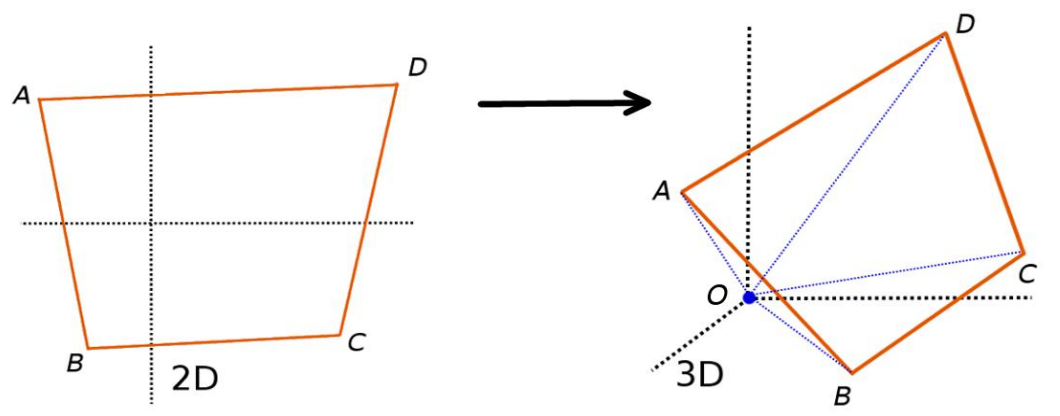

Square plane

virtual 3D polyhedral

$A$

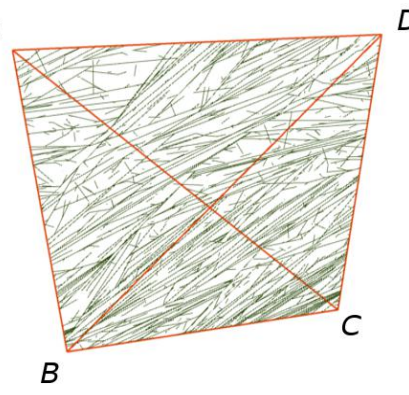

$D$

CMT array (2D)

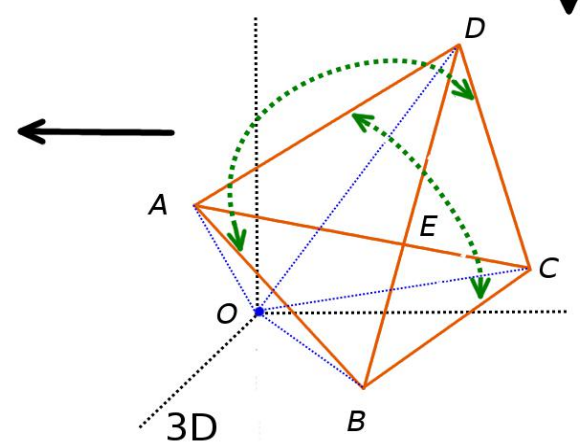

triangulation with PBC

Fig. 2.11. Investigating cortical microtubule dynamics on a two dimensional quadrilateral plane with periodic boundary condition. Green curved arrows indicate microtubule transfer through periodic boundary conditions (PBC). An ordered array of cortical microtubule is shown the with associated orientation of the local order parameters (CMT array).

full triangulation, additionally we triangulated $\square A B C D$ minimally with four triangles $\triangle A B E, \triangle B C E, \triangle C D E$ and $\triangle D A E$. To be noted, all the triangles outside $\square A B C D$ were prohibited from participating in actual simulation and were used only to implement the periodic boundary condition in the microtubule dynamics (see Fig. 2.11, triangulation with PBC), i.e. a microtubule leaving $\triangle A D E$ and entering to $\triangle A D O$ was transferred to $\triangle B C E$ via $\triangle B C O$. Similarly, $\triangle A B O$ and $\triangle D C O$ were used to communicate microtubule dynamics among $\triangle A B E$ and $\triangle D C E$. However, the framework also permits any other boundary conditions. In this way, a two dimensional quadrilateral $\square A B C D$ can be converted into a three dimensional triangulated surface. Now, microtubule dynamics can be simulated as in a three dimensional closed triangulated surface and the corresponding microtubule segments can be projected (CMT array (2D)). To get back the original shape of the quadrilateral $\square A B C D$, the triangles outside $\square A B C D$ and the point $O$ can be deleted. 


\section{Chapter 3}

\section{Parameterization of simulations}

In most of the existing microtubule modelling studies, microtubules were simulated with fitting of the parameters ${ }^{(1)}$ values. The set of parameter values that worked best for establishing the microtubule order were used (Dixit and Cyr, 2004; Allard et al., 2010; Shi and Ma, 2010; Ambrose et al., 2011). Using a mean-field approach, Baulin et al. (2007) were able to reduce the parameter set of five variables into two variables, namely the nucleation rate and the ratio of the speeds of growth and shrinkage. In their mean-field approximation, dynamic instabilities at microtubule plus end were replaced with an average speed of growth and shrinkage. In that model, for deterministically elongating microtubules, the experimentally observed collision induced events were excluded. Instead, the microtubules were instructed to stop growing, only while obstructed by other microtubules. The model proposed by Hawkins et al. (2010), also used a mean-field approximation and reduced the parameter set into one single parameter, namely the "control parameter". The name "control parameter" came from the fact that the value of this parameter allowed them to set in advance the number of interactions among the microtubules. Collision induced events were included and the dynamic instability of the individual microtubules were explicitly considered. The control parameter has been used as input control in several published simulations to assure in advance the formation of microtubule order and array (Tindemans et al., 2010; Deinum et al., 2011; Lindeboom et al., 2013; Tindemans et al., 2014).

For simulation of microtubules on a two dimensional grid, the degree of order and the orientation of the microtubule array were quantified through a two dimensional order parameter (Baulin et al., 2007; Allard et al., 2010; Shi and Ma, 2010), which can capture the dominant alignment direction of the microtubules on the two dimensional plane. This order parameter was used to capture the local order and dominant alignment direction of microtubule on a cubic surface (Ambrose et al., 2011) as well. For a three dimensional shape, on top of capturing the local alignment of the microtubules, to quantify the global order and orientation of microtubule array, a three dimensional global order parameter was introduced by Tindemans et al.

(1) MT parameters: growth/shrinking speed, catastrophe rate, rescue rate, nucleation rate 
(2014). However, the application of the defined global order parameter was limited to mathematically describable three dimensional shapes.

For a given shape, at steady state, the formation of an ordered array of microtubules requires a certain (minimum) number of interactions among the microtubules, i.e a certain value of the control parameter. This domain of the value of the control parameter that assures the formation of a stable ordered microtubule array, was used as the working domain for simulation. A graphical representation of the degree/strength of microtubule order as a function of the control parameter has been used before to find the working domain of simulation (Tindemans et al., 2010; Deinum et al., 2011; Tindemans et al., 2014).

In this chapter, we aim to parameterize our modelling framework, so that it suits best to interpret the simulation output in the biological context. To understand the essential features of the process of microtubule ordering, the modelling framework should be simple, yet powerful enough to capture the major characteristics of the process. This requires the modelling approach to have a clear definition of input control, output measurement, and robustness of the framework against technical variability and variation in input parameters. In Sec. 3.1, we define an input control parameter. In Sec. 3.2 , we formulate two order parameters to quantify microtubule order and array orientation. In Sec. 3.3, we describe a method to find the working domain of simulation. In Sec. 3.4, we test the robustness of the output of our simulation framework against different triangulations.

\subsection{Input control parameter}

A certain number of microtubule interactions are necessary to form an ordered microtubule array, from the initially disordered state. In our simulations, for a given cell (shape), the number of microtubule interactions is controlled by four classes of parameters (see Table 3.1),

Class I: Collision associated parameters $\left(\theta_{z}, p_{\text {cat }}, p_{\text {cross }}\right)$.

Class II: Different Speeds $\left(v^{+}, v^{-}, v^{t m}\right)$.

Class III: Dynamic instability parameters $\left(r_{n}, r_{c}, r_{r}\right)$.

Class IV: Edge-catastrophe associated parameters $\left(\theta_{E d g}^{a v g}, E_{c a t}\right)$.

For a given set of collision associated parameters (Class I), the speeds (Class II, excluding $v^{t m}$ ) and the dynamic instability parameters (Class III) were absorbed into a single control parameter $(G)$, that controls the number of microtubule interactions (Hawkins et al., 2010). A modified version of the control parameter that includes $v^{t m}$, has been used by Deinum et al. (2011). The control parameter was expressed in terms of ratio of two lengths: (a) the 


\begin{tabular}{l|r|r}
\hline Parameter & Description & Value \\
\hline \hline$v^{+}$ & growth speed & $0.08 \mu \mathrm{m} \mathrm{sec}$ \\
$v^{-}$ & shrinkage speed & $0.16 \mu \mathrm{m} \mathrm{sec}^{-1}$ \\
$v^{t m}$ & treadmilling speed & $0.01 \mu \mathrm{mec}^{-1}$ \\
$r_{r}$ & rescue rate & $0.007 \mathrm{sec}^{-1}$ \\
$r_{c}$ & catastrophe rate & variable: $0.003-0.02 \mathrm{sec}^{-1}$ \\
$r_{n}$ & nucleation rate & $10 \mu \mathrm{m}^{-1}$ \\
$\rho_{\text {tub }}$ & finite tubulin pool density (length equiv.) & $40^{0}$ \\
$\theta_{z}$ & angle of zippering & 0.5 \\
$p_{\text {cat }}$ & probability of induced catastrophe & 0.5 \\
$p_{\text {cross }}$ & probability of crossover & $0.001-0.01 \mu \mathrm{m}^{-2} \mathrm{sec}^{-1}$ \\
\hline \hline
\end{tabular}

Table 3.1. Overview of the microtubule parameters and variables with their default values (if applicable). The dynamic instability parameters were used from Vos et al. (2004) and the value of $v^{t m}$ has been approximated from Shaw et al. (2003). The nucleation rate $\left(r_{n}\right)$ has been chosen primarily from Eren et al. (2010), however also varied to maintain sufficient number of microtubules, such that the system always reach to an ordered state. The angle of zippering $\left(\theta_{z}\right)$ and probabilities for zippering $\left(p_{z}\right)$, crossovers $\left(p_{\text {cross }}\right)$ and catastrophes $\left(p_{\text {cat }}\right)$ were deduced (see Fig. 2.5) from DDixit and Cyr (2004) , combining data from MBD-DsRed and YFP-TUA6 labeling. In the biological measurements, no distinction was made between spontaneous catastrophe $\left(r_{c}\right)$ and induced-catastrophe, resulting in a likely high value by an unknown factor. Based on this observed uncertainty and the linear dependency of $G$ on $r_{c}$, we used $r_{c}$ as the primary mean to vary $G$.

average length of microtubules $\left(l_{\text {avg }}\right)$ and (b) the microtubule interaction length $\left(l_{0}\right)$, the microtubule length that is required to enable sufficient

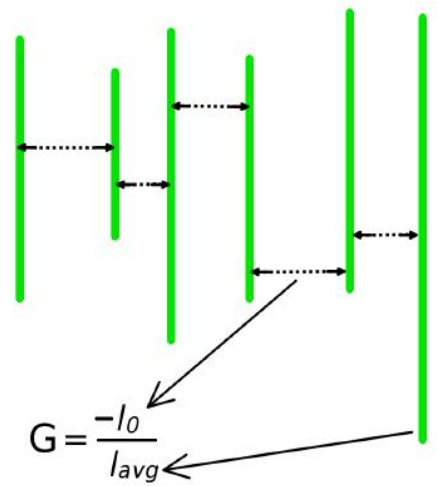

Fig. 3.1. Control parameter $(G)$ that controls the number of microtubule interactions. This is a ratio of two lengths: (a) Average length of microtubules $\left(l_{\text {avg }}\right.$, equivalent to the average length of all the vertical green lines) and (b) Microtubule interaction length ( $l_{0}$, equivalent to the average length of all the horizontal black dashed lines), a measure of the microtubule length that is required to enable sufficient number of interactions.

number of interactions, as (see Fig. 3.1),

$$
G=-\frac{l_{0}}{l_{\text {avg }}}
$$

with,

$$
\begin{aligned}
l_{0} & =\left(\frac{2\left(v^{+}-v^{t m}\right)^{2}\left(v^{-}+v^{t m}\right)}{r_{n} v^{+}\left(v^{+}+v^{-}\right)}\right)^{\frac{1}{3}} \\
l_{\text {avg }} & =\frac{1}{\left(\frac{r_{c}}{v^{+}-v^{t m}}-\frac{r_{r}}{v^{-}+v^{t m}}\right)}
\end{aligned}
$$


The control parameter $(G)$ allowed them to switch on/off the steady state microtubule order by controlling the number of microtubule interactions. The edge-catastrophe associated parameters (Class IV) influence the microtubule catastrophe, thus will effectively get incorporated into the dynamic instability parameter $r_{c}$. As a result, irrespective of the individual contribution of all the different parameters, a system of microtubules can be precisely driven towards ordered/disordered state by modulating the value of $G$. Even in the absence of collisions, the microtubule lengths are intrinsically bounded for $G<0$. As $G$ increases towards 0 , the number of interactions between microtubules also increases, resulting in the formation of enhanced order.

\subsection{Output order parameter}

For a given configuration of microtubules in a particular state of order, we define two order parameters to quantify the strength of order and the orientation of the microtubule array,

1. A local order parameter $\bigcirc_{l}$, which captures the local degree of microtubule order on a part of a surface and the corresponding microtubule array orientation.

2. A global order parameter $\bigcirc_{g}$, which captures the global degree of microtubule order on a three dimensional surface and the corresponding microtubule array orientation.

\section{Measure of local order $\left(\bigcirc_{l}\right)$}

To measure the microtubule order and the orientation of the microtubule array at a local domain of the cell (shape), we defined a local order parameter $\left(\bigcirc_{l}\right) . \bigcirc_{l}$ has a scalar part $(S)$, which is the absolute value of the highest

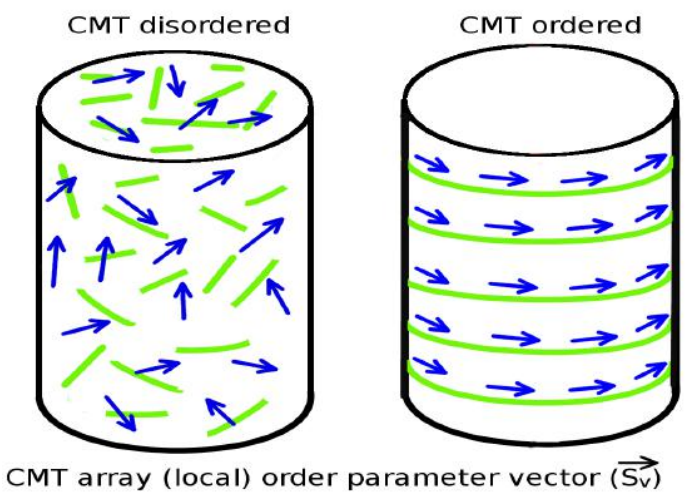

Fig. 3.2. Local order parameter $\left(\bigcirc_{l}\right)$. It has a scalar part $(S)$ and a vector part $\left(\vec{S}_{v}\right.$, blue arrow). In a given configuration of microtubules at a specific local domain: (a) For random orientations of microtubules $S \approx 0$ and distribution of $\vec{S}_{v}$ will be random (left), (b) For well ordered microtubules $S \approx 1$ and distribution of $\vec{S}_{v}$ will be parallel to the major local orientation of the microtubules (right). 
eigenvalue of the tensor $\mathbf{q}^{(2)}$ (see Appendix A.3, Local order parameter tensor), and a vector part $\vec{S}_{v}$, which is the eigenvector corresponding to $S$ (see Fig. 3.2). The scalar part $S$ indicates degree of local order. For randomly oriented microtubules $S \approx 0$ and for well ordered microtubules $S \approx 1$. Local order parameter orientation vector $\vec{S}_{v}$ is parallel to the major local orientation of microtubules and attached to the corresponding local tangent plane.

\section{Measure of global order $\left(\bigcirc_{g}\right)$}

To measure the degree of global order and the global orientation of microtubule array, we defined a global order parameter $\left(\bigcirc_{g}\right)$. $\bigcirc_{g}$ has a scalar part $(R)$, which is the absolute value of the lowest eigenvalue of tensor $\mathbf{Q}^{(2)}$ (see Appendix A.3, Global order parameter tensor), and a vector part $\vec{R}_{v}$, which is the eigenvector corresponding to $R$. The value of $R$ indicates the

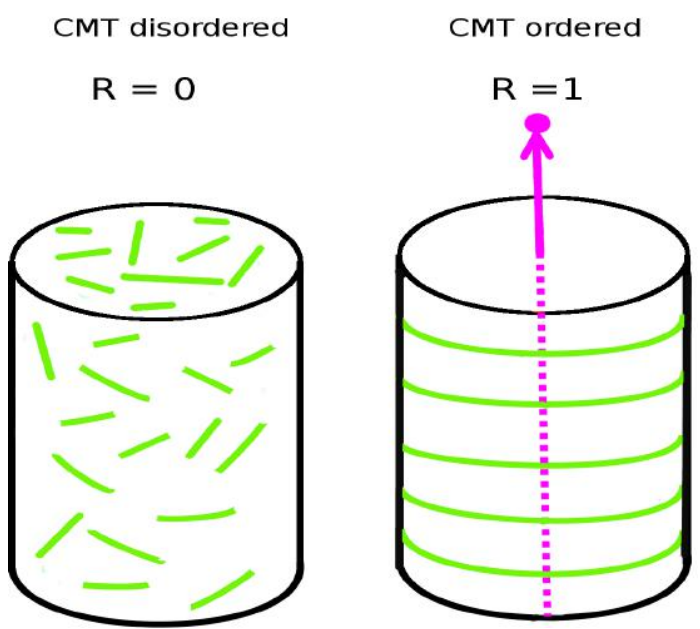

Fig. 3.3. Global order parameter $\left(\bigcirc_{g}\right)$. It has a scalar part $(R)$ and a vector part $\left(\vec{R}_{v}\right.$, magenta arrow). In a given configuration of microtubules: (a) For random orientations of microtubules $R \approx 0$ and orientation of $\vec{R}_{v}$ will be random (left), (b) For well ordered microtubules $R \approx 1$ and orientation of $\vec{R}_{v}$ will be perpendicular to the microtubule array (right). The dot (a filled magenta circle) at the tip of $\vec{R}_{v}$ is a simplified representation of the microtubule array orientation.

degree of global order. For completely random orientation of microtubules $R \approx 0$ and for well organised microtubules that form an array, $R \approx 1$ (see Fig. 3.3). The global order parameter orientation vector $\vec{R}_{v}$ reflects the global orientation of the microtubule array.

\section{Output representation of cell shape and CMT array orientation}

For a given cell shape, we use gray coloured face-tag to indicate the faces shared with neighbouring cells, and white coloured face-tag to indicate non-shared faces (see Fig. 3.4, without CMT array). We have defined two order parameter vectors (local and global) to quantify the orientation of microtubule array. However, unless necessary to show the local orientation of microtubules, we use the global order parameter vector $\left(\vec{R}_{v}\right)$ tips to 
represent the orientation of microtubule array, and as magenta coloured dots. For a given cluster of different simulation configurations, the average array orientation is represented by a magenta coloured arrow, along with an associated microtubule array (see Fig. 3.4, CMT array shown). However,

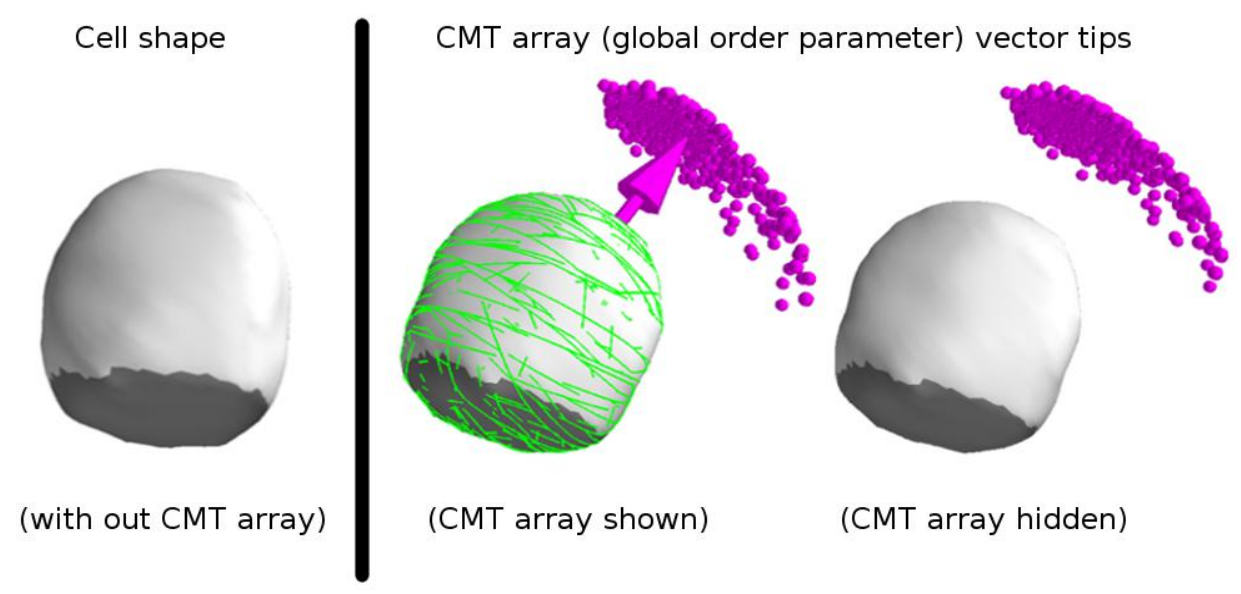

Fig. 3.4. Output representation of cell shape and microtubule array orientation vector (global order parameter vector $\vec{R}_{v}$ ) tips. Representation of the cell shape, where the face shared with other cell is tagged with grey color and the non-shared face is coloured white (Left). Cluster of dots (in magenta color) representing the tips of the global order parameter vector (Right). The arrow represents the average orientation of all the dots in the cluster and associates the corresponding orientation of the microtubule array (CMT array shown). Often, for visual convenience, it is better to hide the average array orientation and the associated microtubule array (CMT array hidden).

for visual convenience, sometimes we hide the average orientation arrow and the associated microtubule array (see Fig. 3.4, CMT array hidden). This last step is especially important for multiple clusters, as drawing an average orientation will be misleading.

\subsection{Working domain}

Unless the number of microtubule interactions are sufficient, the formation of an ordered array of microtubules is not necessarily guaranteed, even when the dynamics of the individual microtubules reaches a steady state. However the control parameter $(G)$ can be appropriately tuned to set the required number of microtubule interactions. To test optimal values of $G$ that ensure a stable value of $R$, we simulated microtubule dynamics on the surface of a sphere for different values of $G$ and measured the corresponding values of $R$. The sphere used in the simulation was of the size of a typical Arabidopsis embryonic cell, as most of the simulations will be performed on realistic cell shapes representing the embryonic cells (Chapter 5) and regular shapes having similar dimensions (Chapter 4). In Fig. 3.5 (right panel), looking at the values of $R$ and the corresponding microtubule array orientations (not shown), we observed that a value of $R \gtrsim 0.70$ was sufficient to declare the associated microtubule array to be ordered, which assures a 

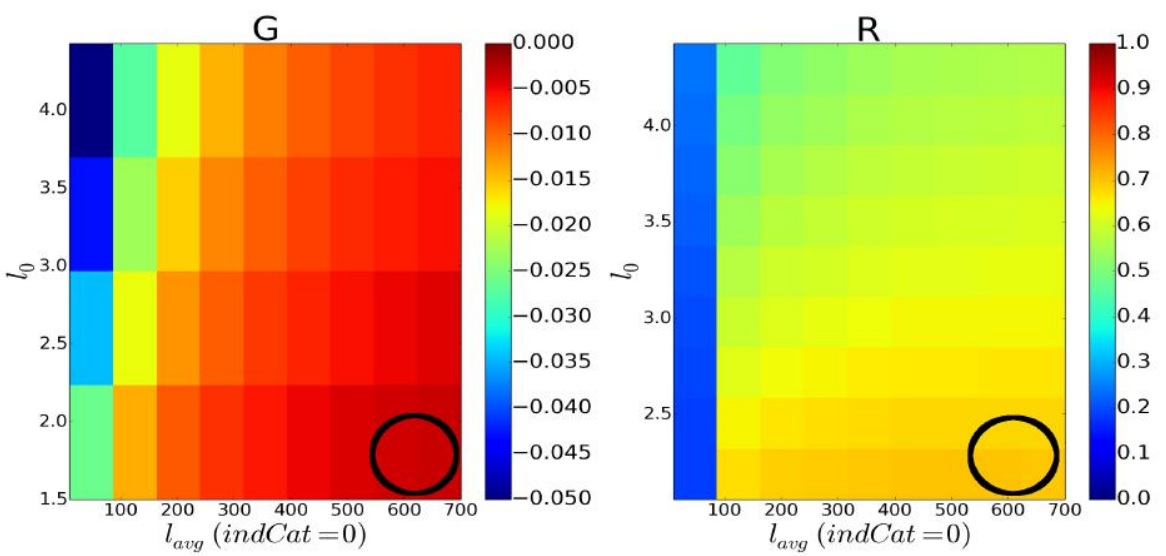

Working domain

Fig. 3.5. Heat map showing the working domain of the values of microtubule-microtubule interaction control parameter $G$, and corresponding values of the global order parameter $R$. We can see a value of $G \lesssim-0.004$ assures sufficient microtubule-microtubule interaction to achieve an order parameter value $R \gtrsim 0.70$ (to see the time evolution, refer to Fig. 3.6 right). The values of $l_{0}$ and $l_{\text {avg }}$ are calculated using Eqn. 3.1This figure is generated by simulating microtubule dynamics on the surface of a triangulated sphere of volume equal to the average volume of an embryonic cell.

stable orientation of the microtubule array at steady state. Following this observation, we defined the domain of the values of $G$ that correspond to the values of $R \gtrsim 0.70$, as the working domain of simulation. In Fig. 3.5 (left panel), looking at the values of $G$, it appeared that the working domain of simulation is $G \lesssim-0.004$. A negative value of $G$ assured that the microtubule lengths were in the bounded regime. The values of $G$ were calculated by using Eqn. 3.1 and the parameter values described in Table 3.1 .

\subsection{Influence of triangulation}

The surface of a given cell (shape) can be approximated by using different triangulation algorithms, with different number of triangles or/and triangles with different shapes. Before simulating microtubule dynamics on the triangulated approximation of the surface of any shape, it has to be ascertained that the simulation results are robust with respect to the choices of triangulation, and produce similar simulation results for different choice of triangulation. In our simulations, the average number of triangles used to approximate a shape is $\simeq 5000$. The surface of the same sphere that was used to find the working domain of simulation was triangulated with five different triangle numbers in the range $1000<T<10000$ ( $\mathrm{T}=$ $1448,2160,2430,4571,8486)$. We simulated microtubule dynamics on the surface of each of these spheres using the same value of input control parameter, $G \approx-0.005$, which falls within the working domain (see Fig. 3.5). As shown in Fig. 3.6, the time evolution of the global order $R$ followed the same 


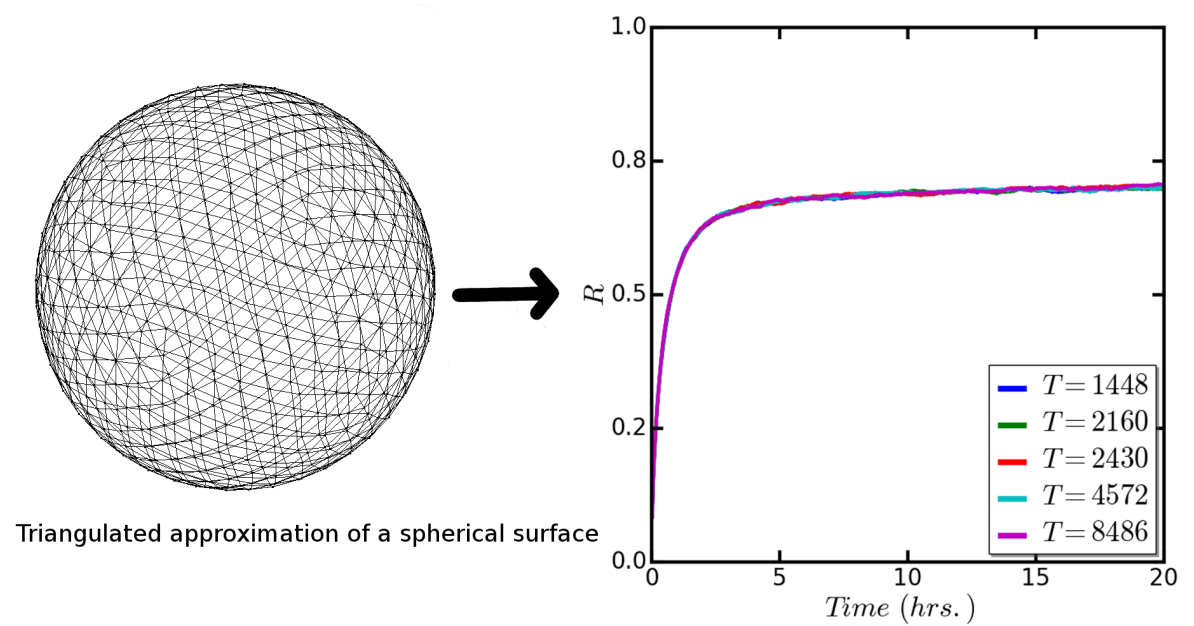

Fig. 3.6. Effect of triangulation of a given surface on cortical microtubule dynamics. Surface of a sphere is triangulated in five different ways with varying number of triangles $(T=1448,2160,2430,4571,8486)$ and each triangle having different shape. Dynamics of microtubules were simulated on the surface of each of these triangulated spherical surface and corresponding time evolution of $R$ is recorded, using same value of input control parameter $G \approx-0.005$, chosen from the working domain (Fig. 3.5) of our simulation. The simulation result shows our developed framework is robust against different triangulated approximation of the surface of the sphere.

trajectory for all the different triangulations. This result clearly showed that the simulation output is robust against variation in triangulation.

\subsection{Summary}

In this chapter, we have parametrized the simulation of microtubule dynamics on triangulated approximations of arbitrary shapes. We used a single input control parameter to control the number of microtubule interactions and explained the significance of this parameter in terms of two length scales, i.e. average microtubule length and microtubule interaction length. To quantify the microtubule order and orientation of the microtubule array, we defined two output order parameters, a local order parameter $(S)$ and a global order parameter $(R)$. We have shown that for a given shape, using a combination of input control parameter $(G)$ and output order parameter $(R)$, it is possible to assure in advance the formation of an ordered array of microtubules (the working domain). We prescribed a schematic representation of the visualization of the microtubule array orientation (see Fig. 3.4), which will be followed as standard representation in the coming chapters. To guarantee the robustness of the simulation output against different choice of triangulation, we compared the simulation outputs of different triangulation, and proved that our simulation technique is robust against variation of triangulation. 


\section{Chapter 4}

\section{Cortical microtubule order as interplay between shape effect and stability rules}

In the plant cell cortex, starting from an initially disorganized state, an ordered array of microtubule is formed (Wasteneys and Williamson, 1989; Kumagai et al., 2001; Shaw et al., 2003; Vos et al., 2003; Ehrhardt and Shaw, 2006). In the absence of a microtubule organizing center, formation of an ordered array of microtubules indicates that collision induced events (Dixit and Cyr, 2004), particularly zippering and induced-catastrophe, play a significant role in the formation of ordered array. Two dimensional attachment of the microtubules to the cell cortex adds a physical constraint to the microtubules, i.e. microtubule can not translate or rotate, but can treadmill (Shaw et al., 2003; Vos et al., 2004). Due to this attachment, the dynamics of microtubule is influenced by cell geometry (Gomez et al. 2016), through a geometrical constraint that depends on the cell shape. The imposed physical and geometrical constraints can change: (1) The interaction length (see Sec. 3.1) of the microtubules, which will influence the rate of collision events; especially the induced catastrophe rate and (2) The locally isotropic growth of microtubules will be transformed into globally anisotropic growth. These constrains are directly coupled with the cell size, which has a direct impact on the microtubule interactions, the smaller is the size of a cell higher is the frequency of induced catastrophe. Plant cell cortex is not always smooth, as insertion of every new division plane creates sharp edges/corners around the perimeter of the inserted division plane. Upon encountering such edges, microtubules undergo edgecatastrophe in a probabilistic way that depends on the curvature of the edge (Ambrose and Wasteneys, 2008). The curvature of a cell-edge is one of the geometrical properties of a given shape, which implies again the role of cell geometry in influencing microtubule dynamics and organization. The plant cell cortex (surface) is composed of multiple cell faces (cell walls) that are inserted at different time points of development. In terms of the distribution of linker/anchoring (stabilizing) proteins (Ambrose and Wasteneys, 2008; Dhonukshe et al., 2003), the newly inserted cell face are likely to be different from the older ones. Such difference might affect the microtubule ordering by offering different order of stabilization to microtubules at different 
cell faces. Taken together, potentially there are at least four factors that might play a role in the self-organization process and the orientation of cortical microtubule array. In this chapter, we aim to address these different contributions independently and sketch a general idea about their right contribution towards the precise orientation of the microtubule array. In Sec. 4.1, we address the contribution of microtubule interactions. In Sec. 4.2, We study the contribution of shape. In Sec. 4.3 we investigate the contribution of edge-catastrophe. Finally, In Sec. 4.4, we test the impact of cell-face induced microtubule stabilization.

\subsection{Contribution of microtubule interactions}

To find out the contribution of microtubule interactions to the order and orientation of the microtubule array, we simulated microtubule dynamics on an open two dimensional quadrilateral plane with periodic boundary condition in the microtubule dynamics. As the shape of a quadrilateral is a two dimensional plane, the microtubule array stays attached to the plane, resulting the global order parameter vector $\vec{R}_{v}$ (see Sec. 3.2) to be always perpendicular to the quadrilateral plane. To bypass this triviality, for quantifying the degree and orientation of the microtubule array on the quadrilateral plane, we preferred to use an average of all the local order parameter vectors $\vec{S}_{v}$ over $\vec{R}_{v}$. To recall, local order parameter vectors $\vec{S}_{v}$

(A)

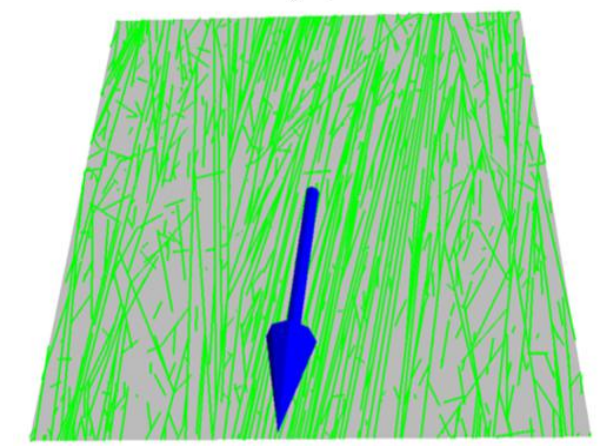

(B)

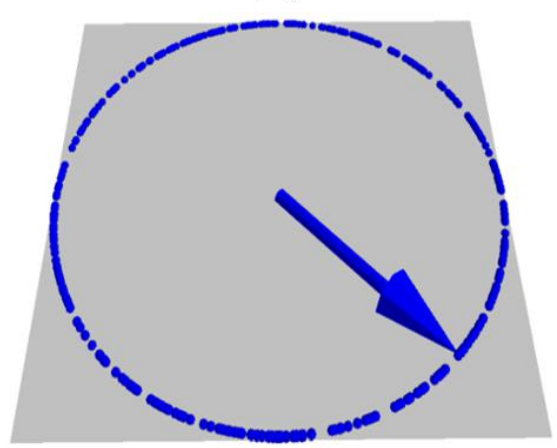

Fig. 4.1. Simulated orientation of microtubule arrays on a square plane with periodic boundary condition on the dynamics of the microtubules. Simulation was performed for $\approx 1000$ different configurations: (A) At steady state, an ordered array of microtubule was formed, with the blue arrow representing the average orientation of the local order parameter vector $\left(\vec{S}_{v}\right)$, and (B) Each dot point represents the tip of an orientation vector associated with a simulation configuration, and parallel to the major alignment direction of the microtubules. The direction of the local order parameter vector was not unique, instead the orientations were apparently completely random.

(see Sec. 3.2) were defined to be attached to each triangle of the triangulated surface and aligned along the direction of maximum microtubule order at the associated triangle. For simulation, we choose a quadrilateral with equal height and width, i.e a square plane of size $80 \mu m \times 80 \mu m$, and allowed microtubule dynamics to continue until the system reached a steady state 
by forming a stable array of microtubules. Our simulation result showed that at steady state, with proper choice of the value of control parameter (see Sec. 3.1), a stable array of microtubules was formed (see Fig. 4.1. CMT array). We performed $(\approx 1000)$ independent simulations and each of them resulted in a stable array of microtubules with a clear dominant alignment direction. However, this dominant alignment diretion of the microtubule array was not unique for all the different realizations, rather the distribution of these dominant alignment directions appeared to be random (see Fig. 4.1). This result suggested that, mutual interactions among the microtubules can drive the system from a disordered to an ordered state, but can not establish a unique direction of order.

\subsection{Contribution of shape}

Interactions among the microtubules are directly influenced by the geometrical constraints imposed by the shape. For example as illustrated in Fig. 4.2 , in a $2 \mathrm{D}$ shape, which is open at the boundary edges, the dynamics of microtubules will be influenced by the type of boundary condition used for a growing/shrinking microtubule. In this case, independent of the shape of the $2 \mathrm{D}$ domain, the global order parameter vector $\left(\vec{R}_{v}\right)$ is always perpendicular to the plane of the 2D shape (Fig. 4.2, 2D shape). However, the same 2D shape when transformed into a quasi 3D shape (Fig. 4.2 , quasi 3D shape), the dynamics of microtubules will remain the same,

(A)

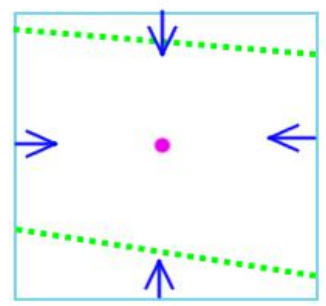

(B)

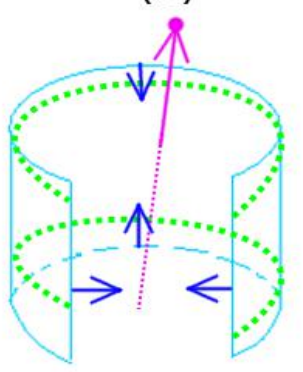

(C)

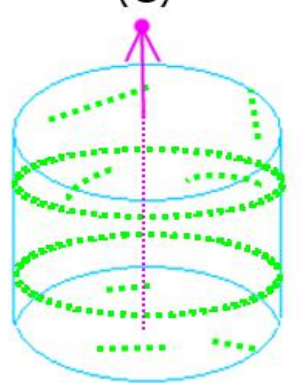

Fig. 4.2. Schematic diagram explaining the influence of shape on microtubule array orientation: (A) For a 2D shape, boundary conditions (boundary edges are indicated by inward blue arrow) are required to propagate the microtubule dynamics. $2 \mathrm{D}$ shape makes the orientation of the global order parameter vector $\left(\vec{R}_{v}\right)$ to be always perpendicular to the plane of the microtubule attachment. This trivial orientation of $\vec{R}_{v}$ is misleading as it doesn't capture any changes in the actual orientation of the microtubule array, (B) The same 2D shape is converted into a quasi 3D shape. In this shape, the orientation of $\vec{R}_{v}$ is no longer trivial, instead depends on the kind of geometrical modification made to transform the quasi $3 \mathrm{D}$ shape into the $2 \mathrm{D}$ shape, and $(\mathrm{C})$ The quasi $3 \mathrm{D}$ shape is converted into a closed $3 \mathrm{D}$ shape. This transformation removes the necessity of boundary condition. In this case, the number of microtubule interactions, hence the orientation of the microtubule array $\left(\vec{R}_{v}\right)$ will be controlled by the shape anisotropy and size of the 3D shape. Here, microtubules are schematically shown by green dotted lines.

but this geometrical modification will result in a non-trivial orientation 
of $\vec{R}_{v}$. Finally, transforming the quasi 3D shape into a closed 3D shape (Fig. 4.2, 3D shape) will influence the orientation of $\vec{R}_{v}$ by controlling the microtubule dynamics in the following way:

1. Any constraints of explicit boundary condition in microtubule dynamics will be removed, allowing the microtubules to grow/shrink along the tracks characterized by the $3 \mathrm{D}$ shape.

2. Microtubules growing along the shorter dimension of the shape will have higher possibility of self-interactions than the larger dimensions.

3. Bigger the size of the shape, smaller will be the number of microtubule interactions.

Here we investigate the contribution of shape on the orientation of the microtubule array by simulating microtubule dynamics on regular shapes. We created different shapes with increased degree of shape anisotropy, where each of these shapes have volumes equal to the volume of a typical Arabidopsis embryonic cell. To perform simulation, the surface of all these shapes was approximated by an appropriately triangulated three dimensional surface.

A sphere is an isotropic shape with infinitely many axes of symmetry. When we simulated microtubule dynamics on the spherical surface, we observed that the microtubule array orientation was not in same direction for the different simulation realizations and apparently completely random (see Fig. 4.3). Due to the isotropic nature of the shape, even though microtubule

(A)

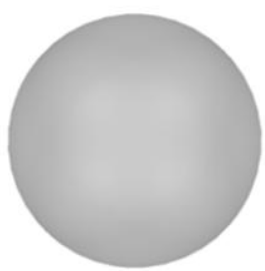

(B)

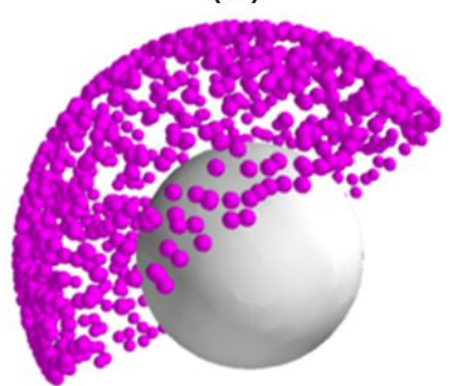

Fig. 4.3. Simulated orientation of microtubule array on spherical surface. Simulation was performed for $\approx 1000$ different configurations: $(\mathrm{A})$ A schematic representation of the sphere, and (B) Each dot point represents the tip of an orientation vector associated with a simulation configuration, and perpendicular to an associated microtubule array. For spherical surfaced the orientation of the microtubule arrays were apparently completely random.

dynamics was influenced by the geometrical constraints, the microtubule array was unable to establish a favoured orientation. Next, we studied the microtubule dynamics on the surface of a rectangular parallelepiped, with dimension $a, b$ and $c$ along the $x, y$ and $z$ axis respectively. Associated 
with each axis, we defined a vector with magnitude proportional to the corresponding dimension of the parallelepiped, i.e. $\vec{p}_{a}, \vec{p}_{b}$ and $\vec{p}_{c}$ with magnitude $a, b$ and $c$, and along the $x, y$ and $z$ axis respectively. We call these vectors as symmetry director (see Appendix A.1, Symmetry directors). As

(A)
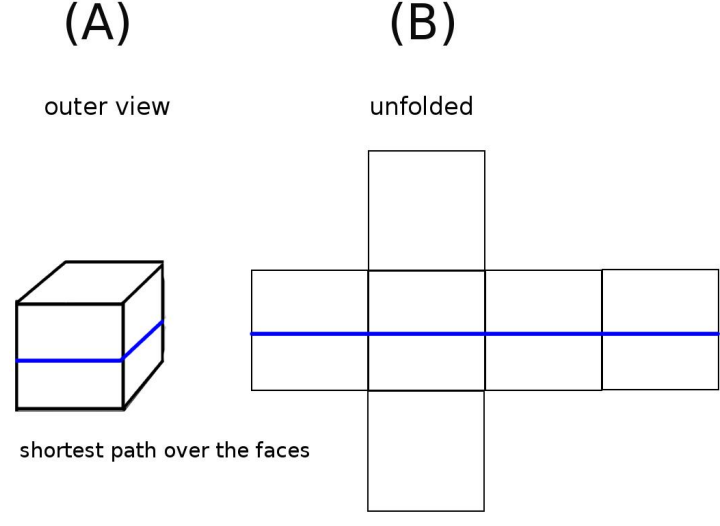

inner view

(C)

(D)

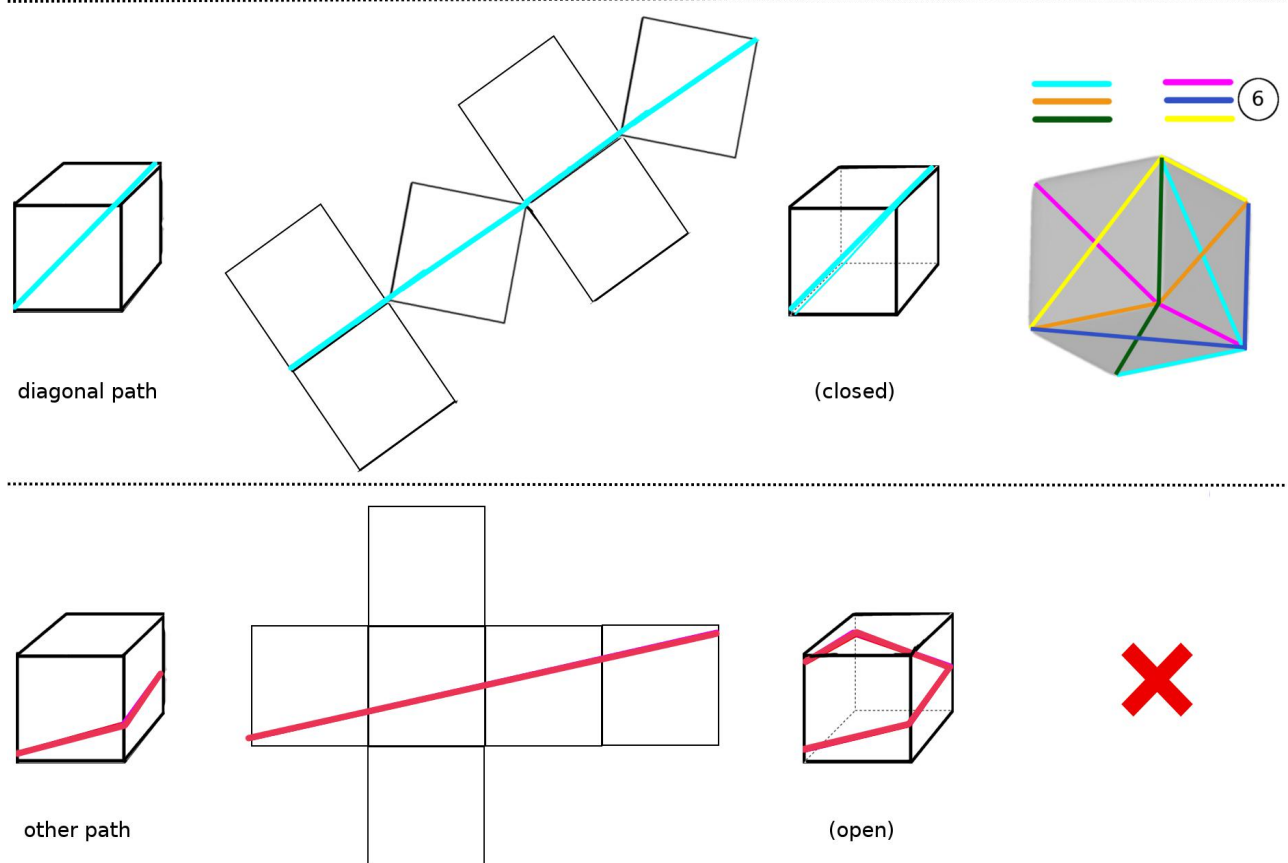

Fig. 4.4. Possible paths of microtubule array formation on a cubic surface. A microtubule path is shown by a coloured line and in three different ways of view: (A) Through the outer surface of the cube, (B) In the unfolded version of the cube surface, (C) Through the inner surface of the cube, and (D) Maximum closed paths that can be drawn through: (I) The shortest path over the faces is three (top panel), (II) Through the diagonal paths is six (mid panel) and (III) Any other closed path is none (bottom panel).

our first attempt of introducing shape anisotropy, we used a rectangular parallelepiped with $a=b=c$, i.e. $\left|\vec{p}_{a}\right|=\left|\vec{p}_{b}\right|=\left|\vec{p}_{c}\right|$ (cube), which meant all the symmetry directors were identical. On this cubic surface. A collection of different simulation realizations gave multiple clusters of the microtubule array orientation. For a given cube, having six faces, a maximum of three 
closed paths (see Fig. 4.4, top panel) can be drawn through the shortest path over the faces. For the same cube, having twelve edges, a maximum of six closed paths (see Fig. 4.4, mid panel) can be drawn through all the possible diagonal paths. There is no other possibility of drawing a closed path (see Fig. 4.4, bottom panel). So, in total nine closed paths can be drawn. In our simulation, exactly in total nine different clusters (see Fig. 4.5) of microtubule array orientation were observed. This result

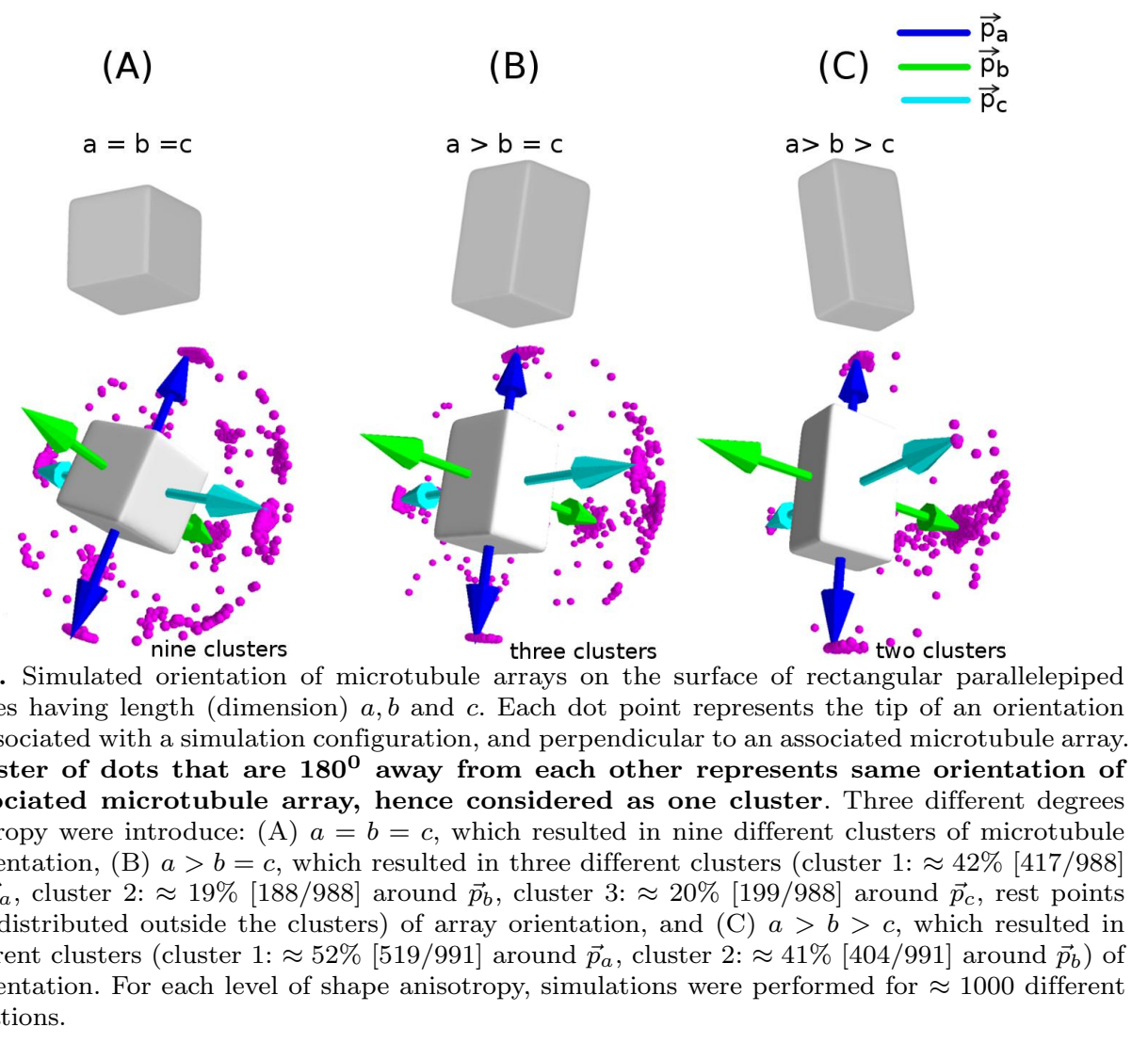

suggested that inherent anisotropic influence of the shape of a cube can lead to favoured orientations of microtubule array, as the orientation of microtubule array reduced from infinite (theoretically random) to nine possibilities. To enhance the strength of shape anisotropy of the rectangular parallelepiped, we choose $a>b=c$, i.e. $\left|\vec{p}_{a}\right|>\left|\vec{p}_{b}\right|=\left|\vec{p}_{c}\right|$, which resulted in $\vec{p}_{a}$ to be the longest symmetry director, leaving the other two symmetry directors shorter and identical. Our simulation result showed this increase in shape anisotropy, further reduced the possible orientation of microtubule arrays from nine possibilities to three possibilities, where the microtubule arrays were formed along the shortest path around the three symmetry directors. The microtubule arrays were formed predominantly around the symmetry director $\vec{p}_{a}$ (cluster: $\approx 42 \%$ ) and with almost identical probabilities around the rest two symmetry directors $\vec{p}_{b}$ (cluster: $\approx 19 \%$ ) and 
$\vec{p}_{c}$ (cluster: $\approx 20 \%$ ) (see Fig. $\left.4.5,(\mathrm{~B})\right)$. As next level of enhanced shape

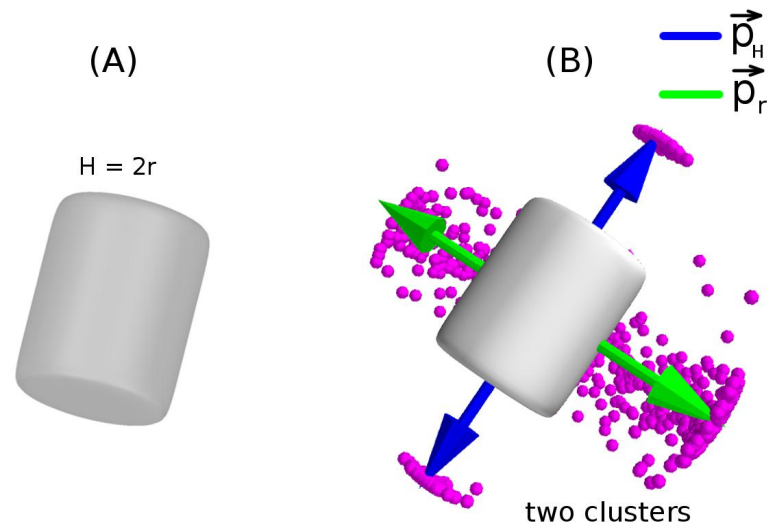

Fig. 4.6. Simulated orientation of microtubule arrays on the surface of a cylinder, with height $H$ and radius $r$ : (A) A schematic representation of the cylinder, and (B) Each dot point represents the tip of an orientation vector associated with a simulation configuration, and perpendicular to an associated microtubule array. The cluster of dots that are $180^{\circ}$ away from each other represents same orientation of the associated microtubule array, hence considered as one cluster. Two different clusters of microtubule array orientation were formed, one around the longest symmetry director $\vec{p}_{H}$ (cluster $1: \approx 45 \%[444 / 996]$ ) and another around the symmetry director $\vec{P}_{r}$ (cluster $2: \approx 55 \%$ [552/996]) that resides on the orthogonal plane of $\vec{p}_{H}$. Simulation was performed for $\approx 1000$ different configurations.

anisotropy, we changed the dimension of the rectangular parallelepiped by setting $a>b>c$, i.e. $\left|\vec{p}_{a}\right|>\left|\vec{p}_{b}\right|>\left|\vec{p}_{c}\right|$. This resulted in all the three symmetry directors to be different, causing further reduction in the possible orientation of microtubule arrays, from three possibilities to two possibilities (see Fig. 4.5, (C)). Particularly, microtubules tend to form an array either around the longest symmetry director $\vec{p}_{a}$ (cluster: $\approx 52 \%$ ), or around the second long symmetry director $\vec{p}_{b}$ (cluster: $\approx 41 \%$ ), but not around the shortest symmetry director $\vec{p}_{c}$ or another arbitrary symmetry director. Finally, we simulated microtubule dynamics on a cylinder with height $H$ (longitudinal dimension) and radius $r$ (radial dimension), where $H=2 r$. For this cylinder, we defined a uniquely oriented symmetry director $\vec{p}_{H}$ with magnitude $\left|\vec{p}_{H}\right|=H$, along the longitudinal dimension and a symmetry director $\vec{p}_{r}$ with magnitude $\left|\vec{p}_{r}\right|=r$, along the radial dimension, which remains invariant in length in the angular range $[0,2 \pi]$, and resides on the orthogonal plane of $\vec{p}_{H}$. Our simulation result showed that microtubules form arrays around two paths, one around the longest symmetry director $\vec{p}_{H}$ (cluster: $\approx 45 \%$ ) and another around $\vec{p}_{r}$ (cluster: $\approx 55 \%$ ) (see Fig. 4.6 ).

Here, we explain the reason behind the preferential orientation of the microtubule array on an anisotropic surface. For a rectangular parallelepiped with dimension $a>b>c$, the length of the shortest closed path around the three symmetry directors $\vec{p}_{a}, \vec{p}_{b}$ and $\vec{p}_{c}$ are $L_{a}=2(b+c), L_{b}=2(a+c)$ and $2(a+b)$ respectively, i.e $L_{a}<L_{b}<L_{c}$ (see Fig. 4.7, (C)). As shorter is the length of a given path, higher is the probability of microtubule self-interactions along that path, therefore $L_{a}<L_{b}<L_{c}$, implies that 
(A)

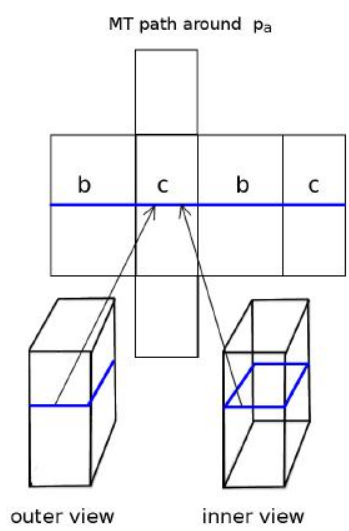

(B)

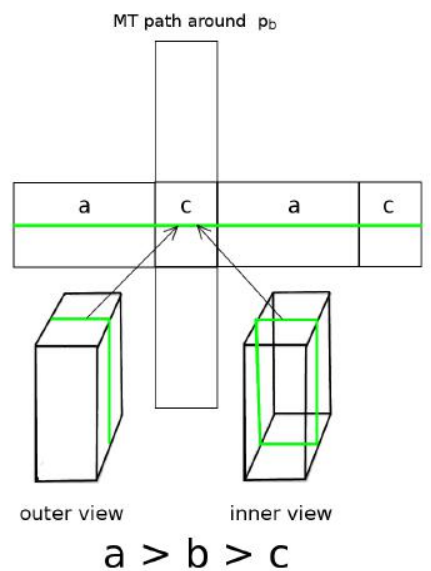

(C)

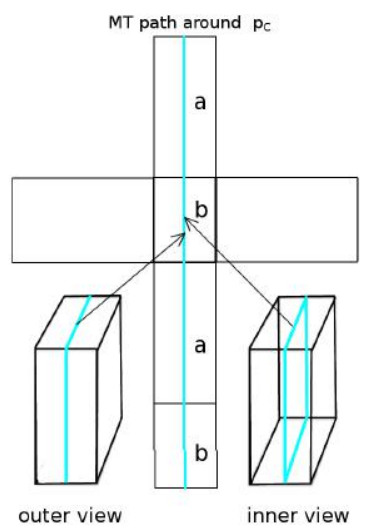

Fig. 4.7. Explanation of the orientational preference of microtubule arrays on an anisotropic shape. To explain the basic principle of the effect of shape anisotropy on microtubule array orientation, a rectangular parallelepiped with edges having lengths (dimension) $a>b>c$ is used in this schematic representation. Microtubule array paths around the three symmetry directors are shown in coloured line - (A) Blue path with length $L_{a}=2(b+c)$ around the longest symmetry director $\vec{p}_{a}$, (B) Green path with length $L_{b}=2(a+c)$ around the second long symmetry director $\vec{p}_{b}$, and (C) Cyan path with length $L_{c}=2(a+b)$ around the shortest symmetry director $\vec{p}_{c}$. The edge length inequality $a>b>c$ implies a corresponding inequality in the microtubule paths $L_{a}<L_{b}<L_{c}$. Simulation result revealed (see Fig. 4.5 (C)) that the probability of array formation around the three symmetry directors decrease from $\vec{p}_{a} \rightarrow \vec{p}_{b} \rightarrow \vec{p}_{c}$, which can be linked to the corresponding microtubule path sequence $L_{a} \rightarrow L_{b} \rightarrow L_{c}$, suggesting that the shorter the length of a given path the higher is the probability of array formation.

the probability of array formation around the three symmetry directors should decrease from around $\vec{p}_{a} \rightarrow \vec{p}_{b} \rightarrow \vec{p}_{c}$. Our simulation prediction of microtubule array formation on regular shapes exactly followed this order.

\section{Biological insight}

In the plant cell, attachment of the cortical microtubules to the cell cortex implies an influence of cell shape/size on microtubule interaction frequencies, thus on the cortical microtubule array orientation. Therefore, a long standing speculation is that cell shape has an influence on the orientation of the cortical microtubule array, and thus on the division plane orientation. Given the variability in the cell shape anisotropy observed within and in between different plant species, our simulation inference of the sensitivity of microtubule array orientation to the shape anisotropy, gives a possible molecular basis towards such speculation.

\subsection{Contribution of edge-catastrophe}

In a high curvature domain, i.e. at an edge of a given shape, the probability of edge-catastrophe $\left(P_{E d g}\right)$ depends on the value of edge-angle $\theta_{E d g}\left(P_{E d g} \sim\right.$ $\left.\theta_{E d g}\right)$. Lets assume for a given shape, a closed path along which microtubules form an array has length $L$ and the path passes through $N$ number of edges. 
If each of the edges has same value of edge-angle, thus the same probability of edge-catastrophe $P_{E d g}$, total probability of edge catastrophe along this path is $P_{t o t}^{(L)}=P_{E d g} N$. Assuming the microtubules are growing with an average velocity $v_{\text {avg }}$, the average time takes to cover the path is $t_{\text {avg }}=\frac{L}{v_{\text {avg }}}$.

(B)

(A)

(C)

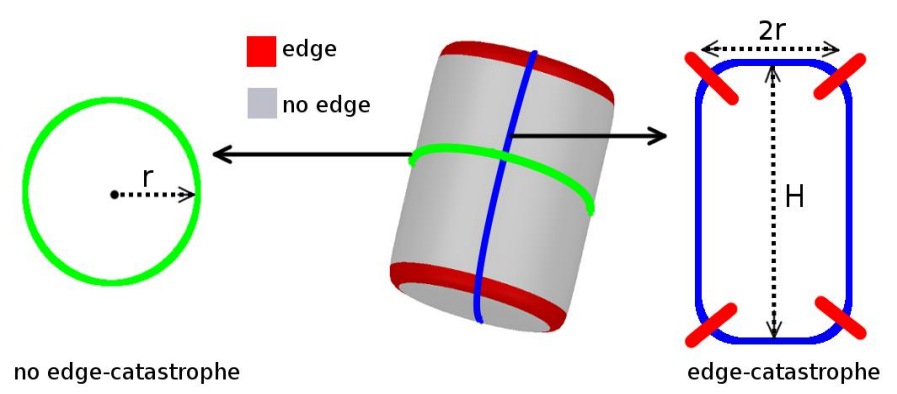

Fig. 4.8. A schematic diagram of the effect of edge-catastrophe on the dynamics of microtubules: (A) In the center, a cylindrical shape with radius $r$ and height $H$ is depicted. We drew two possible paths of microtubule array formation, one along a circular path (green:radius $r$ ) and another along a rectangular path (blue: dimension $2 r \times H$ ), (B) A full view of the circular path that is free of edges, hence no edgecatastrophe, and (C) A full view of the rectangular path that encounters four edges (cutting red lines) and induce edge-catastrophe.

So, the rate of edge-catastrophe along this path is,

$$
P_{E d g}^{(L)}=\frac{P_{t o t}^{(L)}}{t_{a v g}}=\frac{v_{a v g} P_{E d g} N}{L} \sim \frac{N}{L}
$$

Therefore, for a given shape with equal probability of edge-catastrophe $\left(P_{E d g}\right)$ at all the edges (i.e. all the edges have same value of edge-angle $\left.\theta_{E d g}\right)$, the rate of edge-catastrophe along a path is proportional to the ratio of the number of edges $(N)$ along that path and the length of that path $(L)$. In case of the cylindrical shape as illustrated in Fig. 4.8, for the rectangular path $(L=4 r+2 H$ and $N=4$; blue, $(\mathrm{C})), P_{E d g}^{(L)} \sim \frac{1}{2 r+H}$. For the circular path no edge-catastrophe, $P_{E d g}^{(L)}=0$, as this path $(L=2 \pi r$ and $N=0$; green, (B)) does not contain an edge. In this case, microtubules will encounter frequent edge-catastrophe along the rectangular path, but no edge-catastrophe along the circular path. As a result, the probability of microtubule array formation will be higher along the circular path.

Here we address the effect of the rate of edge-catastrophe on the dynamics of microtubules, by simulating microtubule dynamics on all the regular shapes that we considered in Sec. 4.2. For each of these shapes, all the edge-angles are equal $\left(\theta_{E d g} \approx \frac{\pi}{2}\right)$, which allows us to refer to Eqn. 4.1 for understanding and interpreting the simulation results.

We began with the rectangular parallelepiped, with dimensions $a=b=c$ (cube). The edges being of equal length, all the possible closed paths are roughly of same length $L=4 a$ (including the diagonal paths 
of length $2(1+\sqrt{2}) a=4.82 a \approx 4 a)$. Additionally, microtubules following any of these paths encounter same number of edges $(N=4)$, with all the edges having roughly same edge-angle $\left(\theta_{E d g} \approx \frac{\pi}{2}\right)$. According to Eqn. 4.1, microtubules suffer equal rate of edge-catastrophe along these paths, resulting an isotropic distribution of edge-catastrophe rates. Consequently, inclusion of edge-catastrophe in the microtubule dynamics destroyed the nine clusters of microtubule array orientation pattern (simulation without edge-catastrophe, Fig. 4.5, (A)) and produced a completely random distribution (see Fig. 4.9, (A)). Next, we simulated the microtubule dynamics

(A)

$a=b=c$
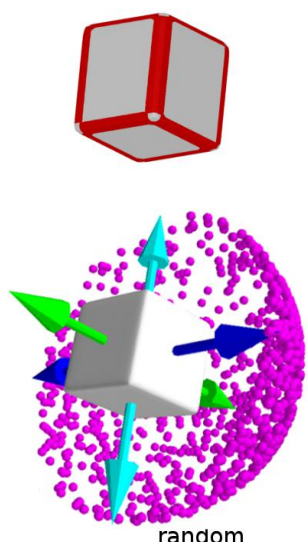

(B)

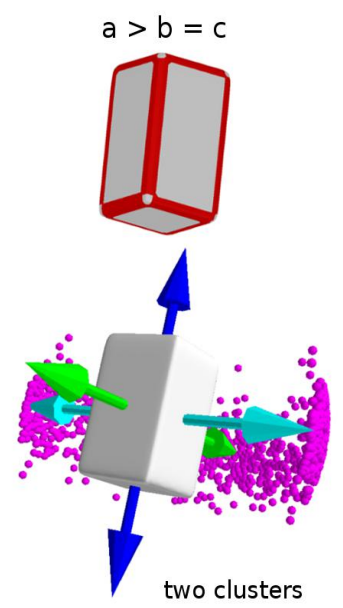

(C) $=\vec{p}_{a}$

$a>b>c$
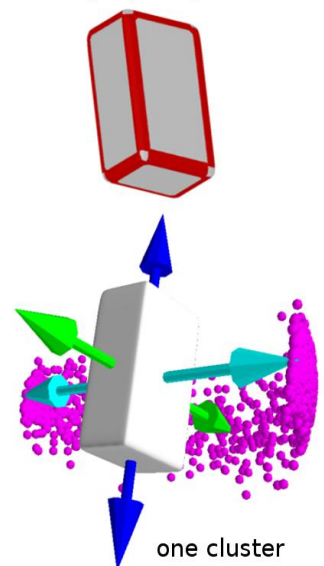

Fig. 4.9. Simulated orientation of microtubule arrays on the surface of rectangular parallelepiped with edges having length (dimension) $a, b$ and $c$, with edge-catastrophe in the microtubule dynamics. Edges of the shapes are coloured red to indicate that in simulation, the microtubules were subjected to edgecatastrophe. Each dot point represents the tip of an orientation vector associated with a simulation configuration, and perpendicular to an associated microtubule array. The cluster of dots that are $180^{\circ}$ away from each other represents same orientation of the associated microtubule array, hence considered as one cluster. Three different degrees of anisotropy were introduce: (A) $a=b=c$, which resulted in apparently random orientation of microtubule array, (B) $a>b=c$, which resulted in two different clusters of approximately equal size (cluster $1: \approx 21 \%$ [210/988] around $\vec{p}_{b}$, cluster $2: \approx 27 \%[273 / 988]$ around $\vec{p}_{c}$, rest points sparsely distributed outside clusters and in the horizontal plane) of array orientation, and (C) $a>b>c$, which resulted in a single cluster (cluster: $\approx 71 \%[706 / 992]$ around $\vec{p}_{c}$, rest points sparsely distributed outside cluster and in the horizontal plane) of array orientation. For each level of shape anisotropy, simulations were performed for $\approx 1000$ different configurations.

on the surface of a rectangular parallelepiped with dimensions $a>b=c$, which implies $\vec{p}_{a}$ to be the longest symmetry director and the other two symmetry directors, i.e $\vec{p}_{b}$ and $\vec{p}_{c}$ to be identical. The path around the longest symmetry director $\vec{p}_{a}$ has length $L_{a}=2(b+c)$ and the paths around the symmetry directors $\vec{p}_{b}$ and $\vec{p}_{c}$ has length $L_{b}=L_{c}=2(a+c)$, as $b=c$. All of these paths contain the same number of edges $N_{a}=N_{b}=N_{c}=4$. As $L_{a}<L_{b}=L_{c}$, using Eqn. 4.1, microtubules were expected to receive more frequent (larger rate) edge-catastrophe along the path around $\vec{p}_{a}$ than the paths around $\vec{p}_{b}$ and $\vec{p}_{c}$, thus avoid forming array around $\vec{p}_{a}$. Indeed, the sim- 
ulation result showed that deviating from producing three clusters around the three symmetry directors (simulation without edge-catastrophe, Fig. 4.5, (B)), in the presence of edge-catastrophe, microtubules avoided making an array around $\vec{p}_{a}$. Instead, the microtubule arrays were formed around $\vec{p}_{b}$ (cluster $1: \approx 21 \%$ ) and $\vec{p}_{c}$ (cluster $2: \approx 28 \%$ ) with almost equal probabilities (see Fig. 4.9, (B)). Simulating microtubule dynamics on the surface of a rectangular parallelepiped with dimensions $a>b>c$, reduced the possible orientations of microtubule array from two clusters (simulation without edge-catastrophe, Fig. 4.5, (C)) to a single cluster (see Fig. 4.9, (C)). In this

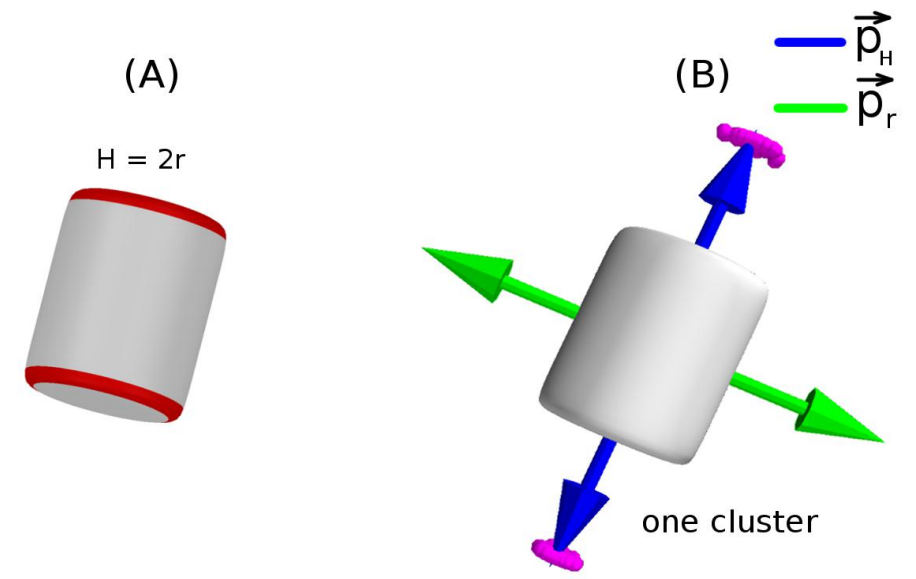

Fig. 4.10. Simulated orientation of microtubule arrays on the surface of a cylinder with height $H$ and radius $r$, and with edge-catastrophe in the microtubule dynamics: (A) A schematic representation of the cylinder with edges coloured in red to indicate that in simulation, the microtubules were subjected to edge-catastrophe, and (B) Each dot point represents the tip of an orientation vector associated with a simulation configuration, and perpendicular to an associated microtubule array. The cluster of dots that are $180^{\circ}$ away from each other represents same orientation of the associated microtubule array, hence considered as one cluster. In our simulation, only a single cluster of array orientation was formed around $\vec{p}_{H}$. Simulation was performed for $\approx 1000$ different configurations.

case, microtubules formed arrays around the shortest symmetry director $\vec{p}_{c}$ (cluster: $\approx 71 \%$ ), which is the path of least rate of edge-catastrophe. The path around $\vec{p}_{a}$, which is the path of highest rate of edge-catastrophe, was completely avoided. The rest fraction microtubule array orientation (cluster: $\approx 29 \%$ ) was sparsely distributed on the orthogonal plane of $\vec{p}_{a}$, i.e horizontal plane. Then, We choose the cylinder with height $H$ and radius $r$ where $H=2 r$. For a cylinder, there is no edge along the path around the longest symmetry director $\vec{p}_{H}$ (path length, $L_{r}=2 \pi r$ and edge number, $N_{r}=0$ ), i.e no edge-catastrophe along this path. However, there exist two edges with high curvature along the path around the other symmetry director $\vec{p}_{r}$ (path length, $L_{H}=H+2 r$ and edge number, $N_{H}=2$ ), resulting a finite rate of edge-catastrophe. Accordingly, in simulation microtubules avoided the path around $\vec{p}_{r}$ and made arrays around the longest symmetry director $\vec{p}_{H}$, giving a single cluster of microtubule array orientations (see Fig. 4.10), instead two (as observed in simulation without edge-catastrophe, Fig. 4.6) 


\section{Biological insight}

Plant cell is composed of multiple faces with an associated edge around the perimeter of the faces. Further, experimental observations have shown that upon encountering a cell-edge, cortical microtubules undergo edgecatastrophe (Ambrose et al., 2011). Through our simulation we discovered that for a given shape, microtubules tend to avoid the paths with higher rate of edge-catastrophe and follow a path of lower rate of edge-catastrophe, thus form an array along that path. Considering the existence of edges that represent the typical characteristic of a plant cell shape, we infer that edge-catastrophe may play significant role in the orientation of the microtubule array, thus on the oriented cell division.

\subsection{Contribution of face stability}

In a given shape, different stability of the microtubules at different cell faces can have significant influence on the orientation of the microtubule array. For example on a spherical surface, due to the isotropic nature of the shape, the orientation of the microtubule array will be random (see Fig. 4.11. (A); also confirmed by our simulation in Fig. 4.3). However, presence

(A)

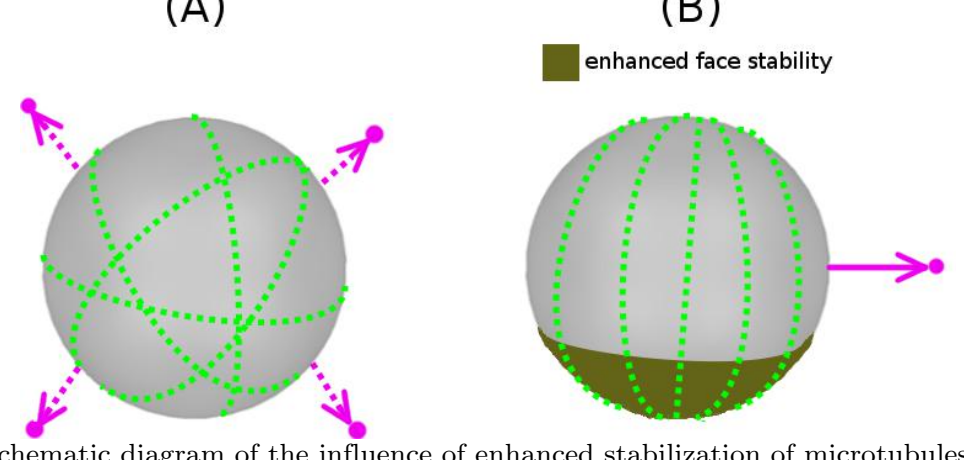

Fig. 4.11. Schematic diagram of the influence of enhanced stabilization of microtubules and the associated array orientation: (A) For a sphere without domain of enhanced microtubule stabilization, we would expect random orientation of microtubule arrays (also showed in our simulation in Fig. 4.3), and (B) In the presence of a domain that enhanced microtubules stabilization, the random orientation of microtubule arrays can converge to a unique orientation. Here, microtubules are schematically shown by green dotted lines.

of a microtubule stabilizing domain can reduce/remove the randomness of the microtubule array orientation (Fig.4.11, (B)). Stabilization of the microtubules will lead to an increased microtubule life time in the stabilizing domain. As a consequence, probability of survival of the microtubules in the direction of the location of the microtubule stabilizing domain will also increase, resulting in formation of microtubule array through the stabilizing domain. 
To test the influence of different face stabilities on the orientation of microtubule array, here we study two cases:

- Case I: For fixed shape anisotropy, test the orientation of microtubule array with respect to the orientation of the microtubule stabilizing domain (face).

- Case II: For fixed orientation of the microtubule stabilizing domain (face), test the orientation of microtubule array with respect to the varying degree of shape anisotropy.

To address Case I, we choose a rectangular parallelepiped, with one alternating face that stabilizes microtubules more than the remaining faces. To study case II, we defined three cylinders of different degree of anisotropy, and for each cylinder we implemented enhanced microtubule stabilization at the bottom circular face. Effect of microtubule stabilization, i.e. average life time of microtubules was modulated via a corresponding modulation in the value of spontaneous catastrophe $\left(r_{c}\right)$, where a lower value of $r_{c}$ results in a higher average life time of the microtubules, i.e. enhanced microtubule stabilization.

First, we studied Case I of a rectangular parallelepiped with dimensions $a>b>c$, where we incorporated enhanced microtubule stabilization at one of the faces perpendicular to the longest symmetry director $\vec{p}_{a}$. In terms of shape, this face has smallest area and hence impose least possible degree of enhanced microtubule stabilization. Our simulation result showed no significant difference in the orientation distribution of the microtubule array (see Fig. 4.12, (A)) from that of default shape simulation (see Fig. $4.5,(\mathrm{C}))$. However, compare to default shape simulation, a small fraction $(\approx 14 \%)$ of microtubule array orientation was shifted from around $\vec{p}_{a}$ and redistributed around the rest two symmetry directors, i.e along $\vec{p}_{b}$ and $\vec{p}_{c}$. Next, we implemented enhanced microtubule stabilization at one of the faces perpendicular to the second large symmetry director $\vec{p}_{b}$. From the shape perspective, this face has second largest area, resulting in the second highest possible degree of enhanced microtubule stabilization. This time a decent fraction of the microtubule array avoided the path around $\vec{p}_{b}$ and redistributed to form arrays around the rest two symmetry directors (see Fig. 4.12, (B)). Incorporation of enhanced microtubule stabilization at one of the faces perpendicular to the smallest symmetry director $\vec{p}_{c}$, resulted in complete avoidance of forming arrays around $\vec{p}_{c}$ and arrays were formed only around each of the other two symmetry directors (see Fig. 4.12, (C)). To note, from the shape perspective, this face has the largest area, resulting in the highest possible degree of enhanced microtubule stabilization. These simulation results indicated that for a shape of fixed anisotropy, microtubules prefer to follow a path that passes through the faces of enhanced 
(A)

$\mathrm{a}>\mathrm{b}>\mathrm{c}$
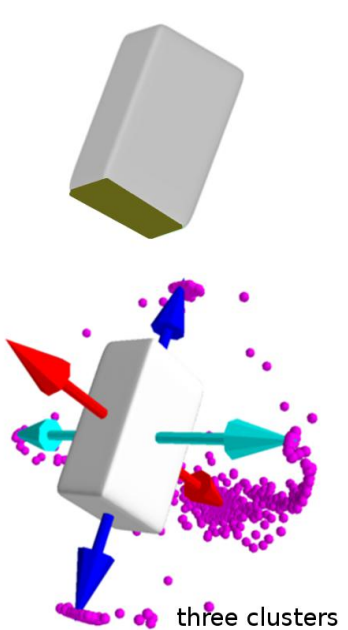

(B)

enhanced face stability
(C)

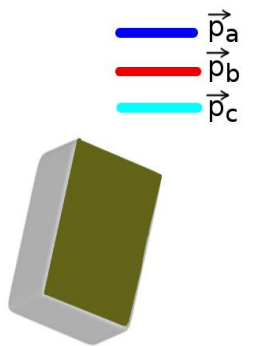

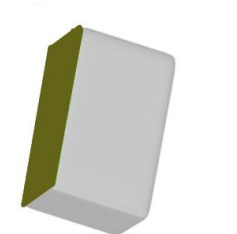
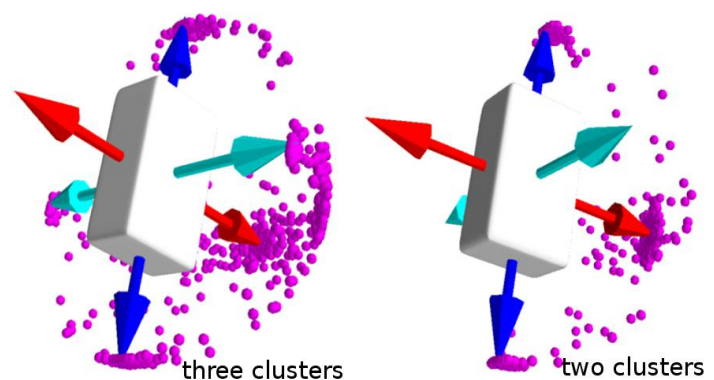

Fig. 4.12. Simulated orientation of microtubule arrays on the surface of rectangular parallelepiped with edges having length (dimension) $a>b>c$, and enhance microtubule stabilization at selected faces. Each dot point represents the tip of an orientation vector associated with a simulation configuration, and perpendicular to an associated microtubule array. The cluster of dots that are $180^{\circ}$ away from each other represents same orientation of the associated microtubule array, hence considered as one cluster. One of six different faces were (alternatively) allowed to provide enhanced microtubule stabilization: (A) enhanced stability at one of the face of the face-pair perpendicular to the longest symmetry director $\vec{p}_{a}$, which resulted in apparently the microtubule array orientation pattern of the corresponding default shape (cluster $1: \approx 38 \%$ [377/988] around $\vec{p}_{a}$, cluster $2: \approx 43 \%$ [424/988] around $\vec{p}_{b}$, cluster $3: \approx 0.05 \%[46 / 988]$ around $\left.\vec{p}_{c}\right),(\mathrm{B})$ enhanced stability at one of the face of the face-pair perpendicular to the second longer symmetry director $\vec{p}_{b}$, which resulted in three clusters of array orientation, where the majority of the microtubule arrays were formed around the symmetry directors $\vec{p}_{a}$ (cluster $\left.1: \approx 49 \%[492 / 994]\right)$ and $\vec{p}_{b}$ (cluster $\left.2: \approx 18 \%[175 / 994]\right)$, the path around $\vec{p}_{c}$ was almost avoided (cluster $3: \approx 12 \%[124 / 994]$ ), and $(\mathrm{C})$ enhanced stability at one of the face of the face-pair perpendicular to the shortest symmetry director $\vec{p}_{c}$, where the formation of microtubule array around $\vec{p}_{c}$ was completely avoided, instead arrayes were formed around the remaining two symmetry directors (cluster $1: \approx 52 \%$ [512/994] around $\vec{p}_{a}$, cluster $2: \approx 48 \%$ [482/994] around $\vec{p}_{b}$ ). Stabilization of microtubules was given via reduced value of dynamic catastrophe $\left(r_{c}\right)$. In this particular simulation we implemented a maximum of 100 times reduction in $r_{c}$ at the face of enhanced stability. For each case of enhanced face stability, simulations were performed for $\approx 1000$ different configurations.

microtubule stabilization. In addition, the greater is the strength of microtubule stabilization at the face, i.e face stability, higher is the possibility of array formation through the corresponding face.

To address case II, we created three cylinders with different shape anisotropy, i.e different radius $(r)$ to height $(H)$ ratio $k=\frac{r}{H}$. A cylinder has two circular faces, at the top and bottom. In our simulation, for $k=2$ (see Fig. 4.13, (A)) and $k=1$ (see Fig. 4.13, (B)), the enhanced microtubule stabilization at the bottom face was able to give a robust orientation of microtubule arrays, which pass through that face. Any other possible path of microtubule array formation was avoided. However, for $k=0.5$ (see Fig. $4.13,(\mathrm{C})$ ), though a major fraction of microtubule array (cluster: $\approx 70 \%$ ) was formed through the face of enhanced microtubule stabilization, a signif- 
(A)

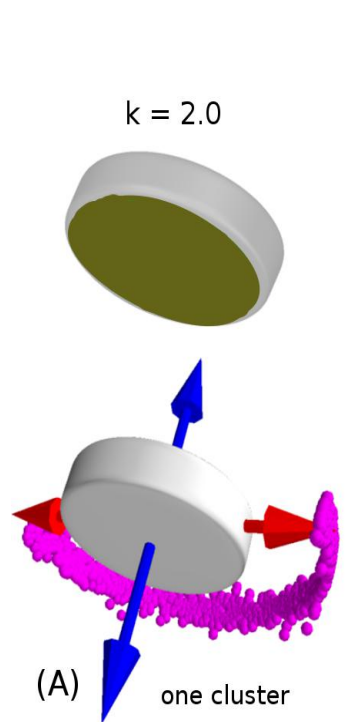

(B)

enhanced face stability
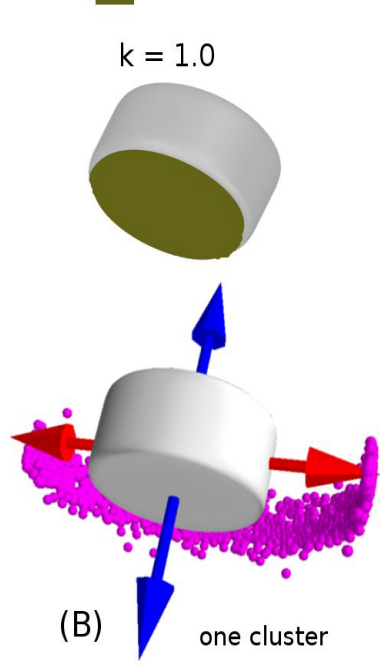

(C)
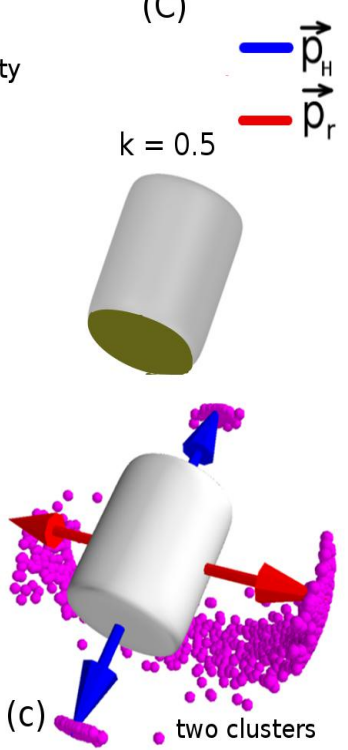

Fig. 4.13. Simulated orientation of microtubule arrays on the surface of cylinders with different degrees of shape anisotropy, i.e. radius $(r)$ to height $(H)$ ratio $k=\frac{r}{H}$, and enhanced microtubule stabilization at one of the circular face (here bottom face). Each dot point represents the tip of an orientation vector associated with a simulation configuration, and perpendicular to an associated microtubule array. The cluster of dots that are $180^{\circ}$ away from each other represents same orientation of the associated microtubule array, hence considered as one cluster. Three different degrees of shape anisotropy (i.e use of different value of $k$ ) were introduced: (A) $k=2$, which resulted in one cluster of microtubule array formation around $\vec{p}_{H},(\mathrm{~B}) k=1$, which again resulted in one cluster of microtubule array formation around $\vec{p}_{H}$, and $(\mathrm{C}) k=0.5$, which resulted in microtubule array formation around each of the symmetry directors $\vec{p}_{H}$ (cluster $1: \approx 70 \%$ [696/992]) and $\vec{p}_{r}$ (cluster $2: \approx 30 \%$ [296/992]). For each degree of shape anisotropy, simulations were performed for $\approx 1000$ different configurations. Stabilization of microtubule was given via reduced value of dynamic catastrophe $\left(r_{c}\right)$. In this particular simulation we implemented a maximum of 100 times reduction in $r_{c}$ at the face of enhanced stability.

icant fraction of microtubule (cluster: $\approx 30 \%$ ) arrays was formed through the alternative curved face as well. These simulation results suggested that the influence of enhanced microtubule stabilization can favour the formation of the microtubule array through the face of enhanced microtubule stabilization, but the influence is limited by the degree of anisotropy of the shape.

\section{Biological insight}

In the context of plant cell division, for example during Arabidopsis early embryogenesis, the new division plane (cell face) is inserted through the previous new cell faces. As division plane orientation follows the orientation of the cortical microtubule array ${ }^{(1)}$, suggesting the cortical microtubule array should form through the previous developmentally new cell faces (cortex). Therefore, our simulation results infer possibility of enhanced microtubule stabilization at the developmentally new cell face.

${ }^{(1)}$ Chapter 1: From microtubule array to cell division 


\subsection{Discussion}

In this chapter, we explored contribution of microtubule interactions, shape anisotropy, edge-catastrophe in microtubule dynamics and the differential microtubule stabilization in the orientation of the microtubule array. Our simulations revealed that mutual interactions among the microtubules can lead to the formation of an ordered array but not sufficient to establish a unique direction of dominant microtubule alignment. We also learn that microtubules can sense the anisotropy of a given shape and respond to it by forming the microtubule arrays along some selective paths. We found that microtubules avoid paths with higher rate of edge-catastrophe and find the smoothest possible path to form an array. Another interesting finding is while enhanced stabilization of microtubules in a particular domain has the potential to favour a particular orientation, anisotropy of the shape can delimit this effect. These simulation predictions brings an important insight towards the understanding of cortical microtubule array formation in the plant cell cortex. We can perceive the role of cell shape and microtubule stabilizing factors in the formation of oriented microtubule array, thus the division plane orientation. 


\section{Chapter 5}

\section{Explaining the orientation of Arabidopsis embryonic cell division planes}

Arabidopsis embryogenesis starts with the asymmetric division of the zygote, creating a smaller apical and larger basal cell. After division of the zygote, the apical cell gives rise to the embryo lineage that generates most of the plant body, whereas the basal cell produces the short-lived suspensor lineage (Jürgens, 2001; Wendrich and Weijers, 2013). The subsequent divisions

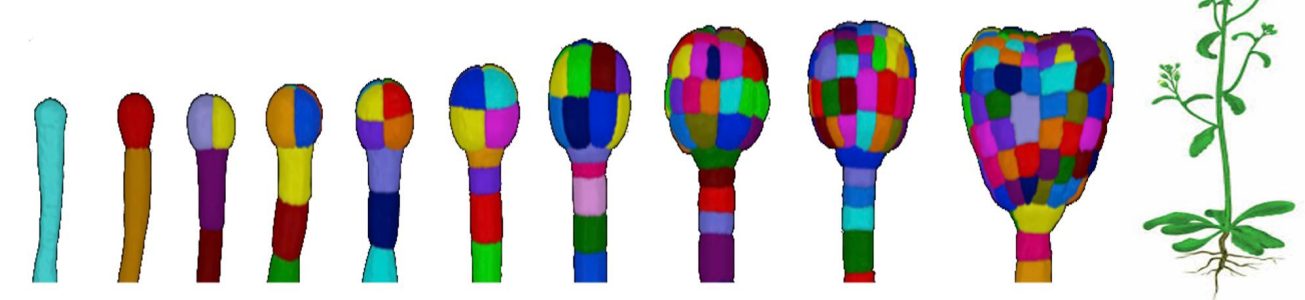

Fig. 5.1. Precise orientation of cell division plane during early embryogenesis of Arabidopsis form the structured layer of cells, which plays a crucial role in the post-embryonic morphogenesis of the entire plant body. The image is adapted from Yoshida et al. (2014).

that take place in the suspensor cells are horizontal, resulting in a single layered connecting structure to maternal tissues. In the early stages of Arabidopsis embryogenesis, the different stages of the apical cell derived embryo are named after the number of cells in the pro-embryo (Jürgens, 1995). During these transitions, the apical cell goes through divisions with highly variable orientations of the division plane, without significant growth in cell size/volume (see Fig. 5.1). In the later stages, growth significantly increases and becomes anisotropic. The basal suspensor cell and its next generations go through anisotropic expansion, where the divisions are always perpendicular to the principal growth direction. Plant cells do not move with respect to each other, causing the morphology of the plant to be determined by the oriented cell division and controlled cell expansion. In the absence of growth, oriented divisions precisely pattern the tissue boundaries and establish cell layers that will be propagated to make the plant morphogenesis efficient. It is imperative to understand the rules of 
early oriented cell division, as these rules will define the tissue architecture that underpins plant morphogenesis during anisotropic growth phase.

Based on different instances of observed division plane orientations, several geometric rules for oriented cell division have been proposed (Hofmeister, 1863; Sachs, 1878; Errera, 1888; Besson and Dumais, 2011). However, none of these rules match the actual embryonic cell division patterns. Any geometric rule should be supported by mechanisms based on cellular components involved in cell division plane orientation. Higher plants establish a highly ordered array of microtubules at cell cortex, and the orientation of the division plane follows the orientation of the cortical microtubule array. The preprophase band (PPB), a dense ring of microtubules formed through the aggregation of the oriented sparse array of the cortical microtubules predicts the insertion site of the new division plane (Vos et al., 2004; Cleary, 2001; Dixit and Cyr, 2002; Mineyuki, 1999; Molchan et al., 2002; Pickett-Heaps et al., 1999; Valster et al., 1997). It has been recognised that this is an important clue towards the molecular understanding of oriented cell division in plant cell (Staehelin and Hepler, 1996).

An ordered array of microtubules is formed through interactions of large numbers of spatially distributed microtubules. Each individual microtubule has their own intrinsic stochastic dynamics, and at the same time they interact with each other through collisions, namely zippering, crossover and induced catastrophe (Dixit and Cyr, 2004). The inherent stochasticity in the dynamics and the complexity that arises through the interactions, make the experimental understanding of the self-organisation process of cortical microtubules difficult. However, in some existing experimental studies the dynamic instability parameters (Shaw et al., 2003; Chan et al., 2003; Dhonukshe and Gadella, 2003; Vos et al., 2004) and the probability of edge-catastrophes were measured (Ambrose and Wasteneys, 2008). Using a combination of theoretical (Hawkins et al., 2010) and computational studies (Tindemans et al., 2010, 2014), we developed a novel modelling framework (see Chapter 2), that uses the existing experimental data and simulate microtubule dynamics using meaningful biochemical parameters on realistic plant cell shapes (cortex). We mathematically described an order parameter (see Chapter 3), to quantify the orientation of simulated arrays of microtubule in steady state. The simulated orientation of the microtubule array can be used to explain the division plane orientation.

In this chapter, we aim to explain the oriented cell divisions during Arabidopsis early embryogenesis in the $b d l$ mutation, which has compromised auxin signalling. We focus on the first five division cycles of the zygote, i.e. zygotic cell and 1,2,4 and 8-cell stages of embryo development and the suspensor cells. We found in Sec. 5.1 that cell shape and edge-catastrophe in microtubule dynamics can generate a robust orientation of microtubule array. Using a combination of experiment and simulation, in Sec. 5.2, 
we show that the similar condition is sufficient to produce microtubule array pattern that explains the observed division plane pattern during early embryonic development, observed in the bdl mutation. In Sec. 5.3, we infer the possible role of anisotropic growth/stress in microtubule dynamics, thus on the associated array orientation. Finally, in Sec. 5.4, we propose a minimal mechanism for the formation of the preprophase band (PPB).

\subsection{Interplay between shape anisotropy and and edge catastrophe predicts a robust orientation of division plane}

Our simulation of microtubule dynamics on regular shapes such as sphere, cube, cylinder etc., showed that shape anisotropy plays a crucial rule in reducing number of microtubule array orientations to a few finite possibilities (see Chapter 4). At the early stages of embryogenesis, all the cell shapes from
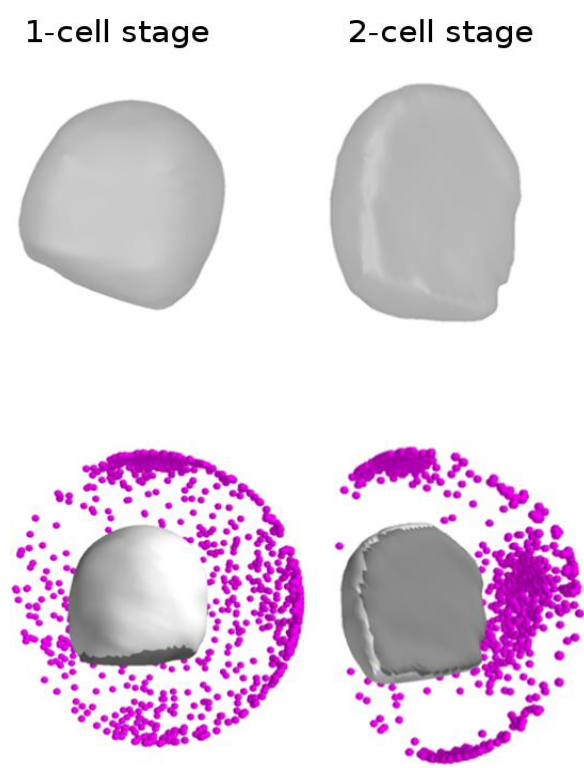
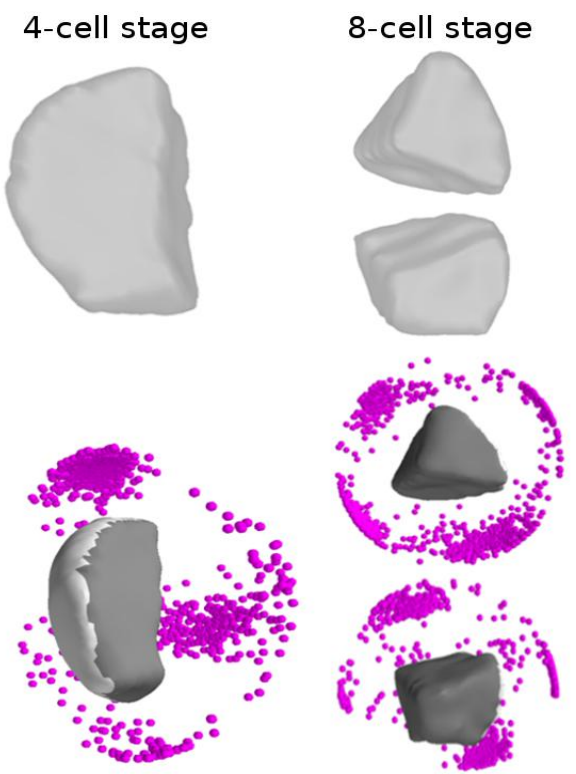

Fig. 5.2. Simulated orientation of microtubule array on default embryonic cell shapes of Arabidopsis. Simulations were performed for $\approx 1000$ different configurations. Each dot point represents the tip of an orientation vector associated with a simulation configuration, and perpendicular to an associated microtubule array. The cluster of dots that are $180^{\circ}$ away from each other represents same orientation of the associated microtubule array, hence considered as one cluster. For each of the different cell stages (1-cell stage, 2-cell stage, 4-cell stage and 8-cell stage), we found two distinct cluster of microtubule array orientation. Simulations were performed on cell shapes that were imaged under normal (wild type) condition. Cell templates used in these simulations were provided by Saiko Yoshida and Dolf Weijers (WUR). For statistical reason, we also performed simulations on the other partner pairs and the results were similar.

$1 \rightarrow$ 8-cell stages are anisotropic. This prompted us to simulate microtubule dynamics on these default cell shapes, to study the cell shape induced possibilities of microtubule array orientations. For simulations, we used 
triangulated approximations of cell surfaces from the 1-cell stage, 2-cell stage, 4-cell stage and 8-cell stage of embryonic development. In all the

1-cell stage

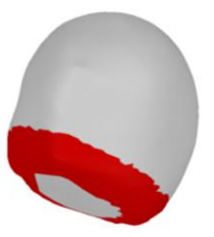

2-cell stage
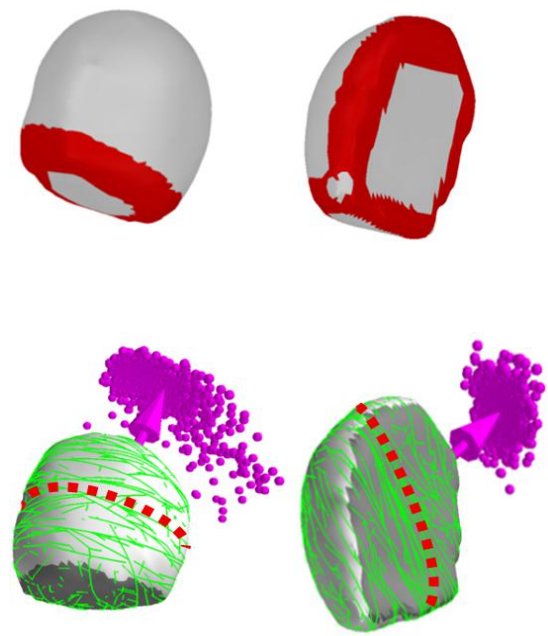

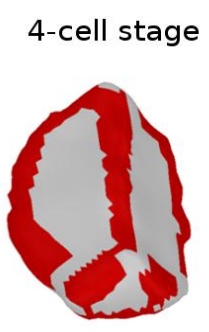

8-cell stage

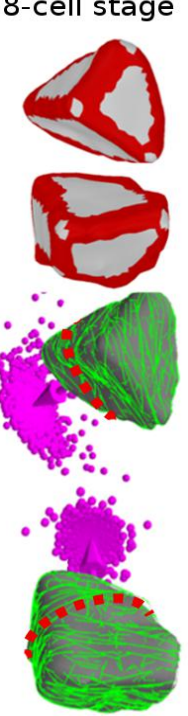

Fig. 5.3. Simulated orientation of microtubule array on embryonic cell shapes of Arabidopsis with edgecatastrophe in microtubule dynamics. Simulations were performed for $\approx 1000$ different configurations. Each dot point represents the tip of an orientation vector associated with a simulation configuration, and perpendicular to an associated microtubule array. For each of the different cell stages (1-cell stage, 2-cell stage, 4-cell stage and 8-cell stage), we found one unique cluster of microtubule array orientation. The arrow vector is drawn through the mean position of all the dots in a cluster and represents the mean orientation of the associated microtubule array. Cell edges are coloured red to indicate that in simulation microtubules were subjected to edge-catastrophes. The red dotted line represents predicted average alignment direction of the associated microtubules, thus the division plane orientation. Simulations were performed on cell shapes that were imaged under normal (wild type) condition. Cell templates used in these simulations were the same as used in Fig. 5.2

four different cell stages, the simulated orientation of microtubule arrays predicted two different possibilities of microtubule array orientation (see Fig. 5.2). This result indicates an important contribution of cell shape in delimiting the oriented divisions to two possibilities. However, the experimentally observed unique orientation of division plane suggests involvement of other factors, which helps to reduce the shape-induced two possibilities of microtubule array orientation into one unique orientation. Experimental observation indicates that microtubules undergo edge-catastrophe at cell edges with high curvature (Ambrose et al., 2011). When we simulated microtubule dynamics with this cell-edge induced edge-catastrophe, our simulation on the same set of cell shapes showed one robust orientation of microtubule array (see Fig. 5.3). Incorporation of edge-catastrophe was sufficient to obtain one unique microtubule array orientation for all cell shapes taken from $1 \rightarrow 8$-cell stages. This result suggested that with general assumptions on microtubule dynamics, but without any genetic/developmental cue, early embryonic cells already possess a definite preference for orienting the microtubule array, and by inference, the division plane. However, note 
Explaining the orientation of Arabidopsis embryonic cell division planes

that the simulated orientation of microtubule array was not the same as the experimentally observed normal (wild type) orientation of the cell division plane, except during the $2 \rightarrow 4$-cell stage transition of embryo development.

\subsection{Cell shape anisotropy and edge catastrophe are sufficient to recreate the $b d l$ cell division patterns}

Existence of genetic control in division plane orientation was described by Hamann et al. (1999), where suppression of the transcriptional auxin response by a nondegradable version of the BDL suppressor protein switched $18 \%$ of the division planes during $1 \rightarrow 2$-cell stage transition. Recent quantification of cell division orientation during early embryogenesis revealed that genetic control overrides geometric division rules (Yoshida et al., 2014). In our recent experimental data, we observed an additional role of auxin in orientation of division plane, as misexpression of a nondegradable version of $b d l(R P S 5 A>>b d l)$ resulted in noticeably tilted horizontal division during $4 \rightarrow$ 8-cell stage transition, in contrast to the sharp horizontal division that happens in normal (wild type) condition (observation: Thijs de Zeeuw, WUR). Such deviations in division plane orientation suggests that BDL mediated auxin signalling is required as a developmental input during early embryonic development. Accumulation of defects became prominent during the $8 \rightarrow 16$-cell stage transition, when almost all cells divided in different division planes compared to the precisely controlled vertical divisions in wild type condition. The differences in cell division pattern between wild type and in the $b d l$ mutated case suggests an active role of auxin in orienting the cell division plane. As our simulations did not account for any transcriptional auxin response, a comparison with the $b d l$ mutated division pattern was appropriate to test whether our simulation rules recreated the $b d l$ division pattern.

Following the same protocol as described in Yoshida et al. (2014), we generated $b d l$ lines and imaged the different cell stages of embryonic development. We performed three dimensional segmentation of the images to precisely quantify the orientation of the division planes (see Fig. 5.4). Because the location and orientation of the newly inserted division plane in parent cell plays an important role on the shape of daughter cells, we compared the shapes of the different cell stages in wild type vs $b d l$ embryos during $1 \rightarrow 2 \rightarrow 4 \rightarrow 8$-cell stage transitions. For each cell-stage, we found a difference in shape. Simulated array of microtubule, with edge-catastrophe in microtubule dynamics, correctly predicted the horizontal division plane orientation for $1 \rightarrow 2$-cell stage transitions in $b d l$ mutation (though in experiment only $\approx 18 \%$ horizontal division was reported (Hamann et al., 1999)) 


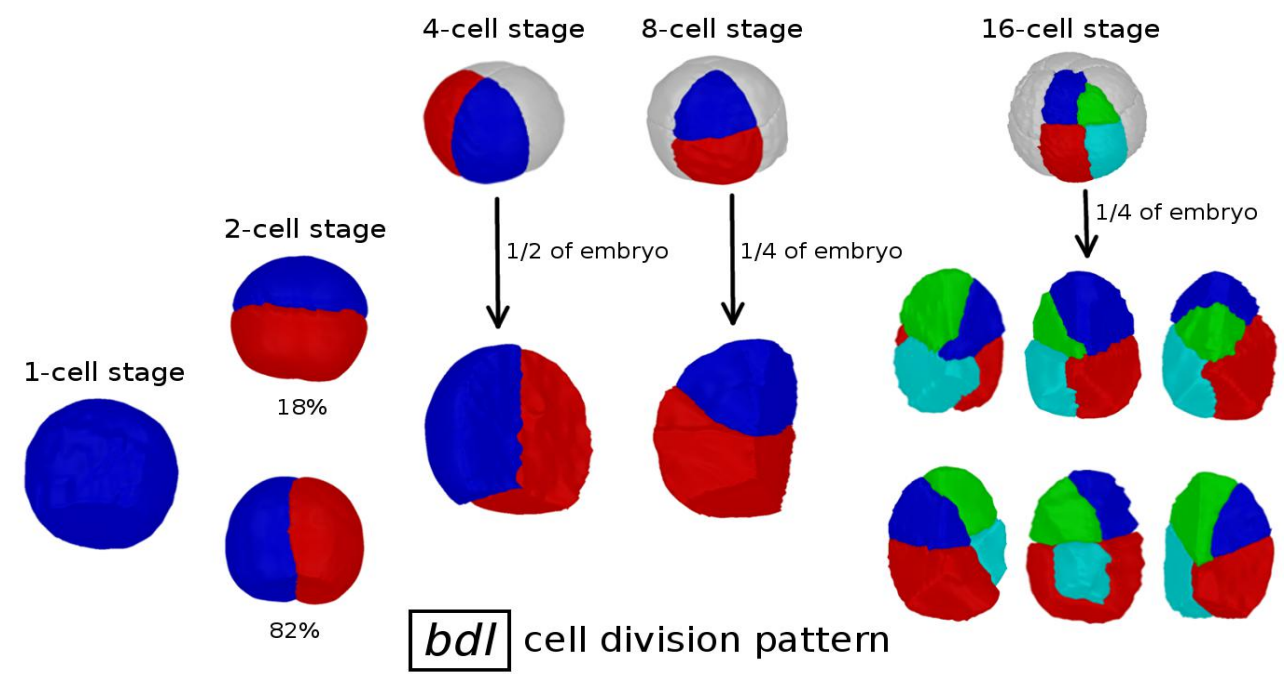

Fig. 5.4. Cell division pattern in bdl embryos of Arabidopsis. During $1 \rightarrow 2$-cell stage transition in most cases $(\approx 82 \%)$ the cell division occurs vertically, however a noticeable fraction $(\approx 18 \%)$ go through horizontal division as well. The $2 \rightarrow 4$-cell stage transition shows vertical division. During $4 \rightarrow 8$-cell stage transition, a tilted horizontal division is observed. The $8 \rightarrow 16$-cell stage transition leads to random orientation of division. Cell templates used in these simulations were provided by Thijs de Zeeuw (WUR).

and the vertical division plane orientation for $2 \rightarrow 4$-cell stage transition. Next, we compared the simulated orientation of microtubule array with the
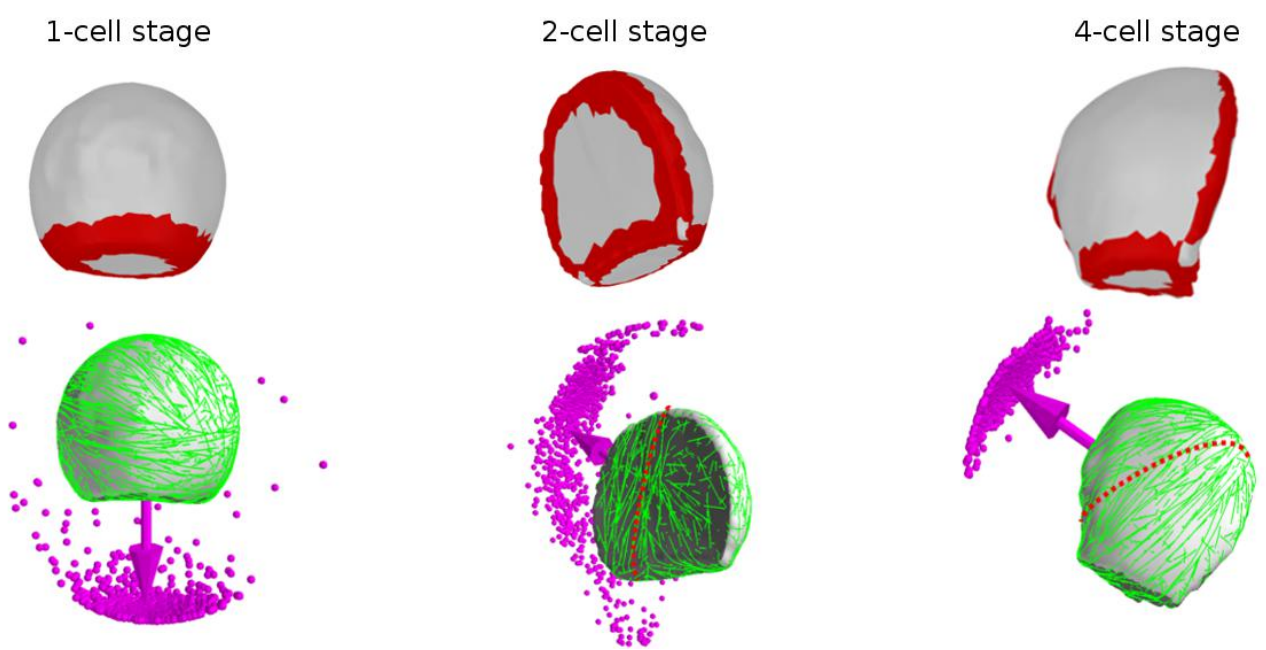

Fig. 5.5. Simulated orientation of microtubule array on embryonic cell shapes of Arabidopsis for $1 \rightarrow 2 \rightarrow 4 \rightarrow$ 8-cell stage transitions, with edge-catastrophe in microtubule dynamics. Simulations were performed for $\approx 1000$ different configurations. Each dot point represents the tip of an orientation vector associated with a simulation configuration, and perpendicular to an associated microtubule array. For each of the different cell stages (1-cell stage, 2-cell stage, 4-cell stage and 8-cell stage), we found one unique cluster of microtubule array orientation. The arrow vector is drawn through the mean position of all the dots in a cluster and represents the mean orientation of the associated microtubule array. Cell edges are coloured red to indicate that in simulation microtubules were subjected to edge-catastrophes. The red dotted line represents predicted average alignment direction of the associated microtubules, thus the division plane orientation. Simulations were performed on cell shapes that were imaged in the $b d l$ mutation. Cell templates used in these simulations were provided by Thijs de Zeeuw (WUR).

division plane orientation at the $4 \rightarrow 8$-cell stage transition state. The array 
orientation correctly predicted the experimentally observed tilted horizontal orientation of cell division. Noteworthy, the experimental observation of

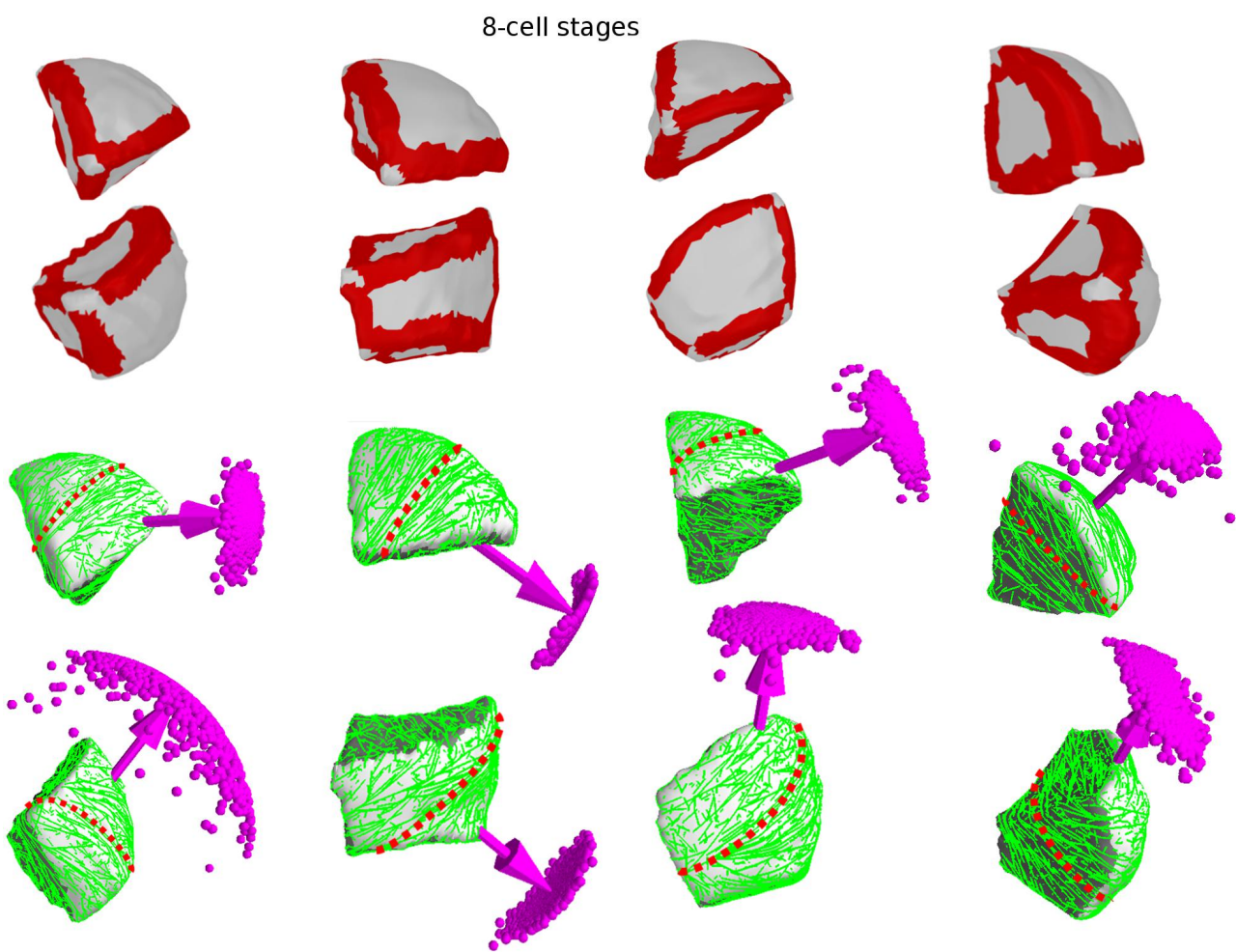

Fig. 5.6. Simulated orientation of microtubule array on embryonic cell shapes of Arabidopsis for $8 \rightarrow 16$-cell stage transition, with edge-catastrophe in microtubule dynamics. Simulations were performed for $\approx 1000$ different configurations. Each dot point represents the tip of an orientation vector associated with a simulation configuration, and perpendicular to an associated microtubule array. For each instances of 8-cell stage cell shapes, we found one unique cluster of microtubule array orientation. The arrow vector is drawn through the mean position of all the dots in a cluster and represents the mean orientation of the associated microtubule array. Cell edges are coloured red to indicate that in simulation microtubules were subjected to edge-catastrophes. The red dotted line represents predicted average alignment direction of the associated microtubules, thus the division plane orientation. Simulations were performed on cell shapes that were imaged in the $b d l$ mutation. Cell templates used in these simulations were provided by Thijs de Zeeuw (WUR).

the highly aberrant division plane orientation during $8 \rightarrow 16$-cell stage transitions were also reflected in our simulations, on the different instances of 8-cell stage cell shape templates (see Fig. 5.6). We next tested whether the resulting orientation of the microtubule array in aberrant $b d l$ 8-cell stage cell shapes gave rise to the division planes actually seen in $b d l$ mutants during $8 \rightarrow 16$-cell stage transition. To address this question, we reconstructed mother cells in 8-cell $b d l$ embryos by merging the corresponding 16-cell stage daughter pair of cells and simulated microtubule dynamics on that mother cell. The simulated array orientation predicted exactly the division plane orientation of the mother 8-cell stage cell (see Fig. 5.7).

However, during $1 \rightarrow 2$-cell stage transition the prediction was partially correct, as simulation with edge-catastrophe predicted only horizontal division (see Fig. 5.5, 1-cell stage) whereas instances of both aberrant horizontal 


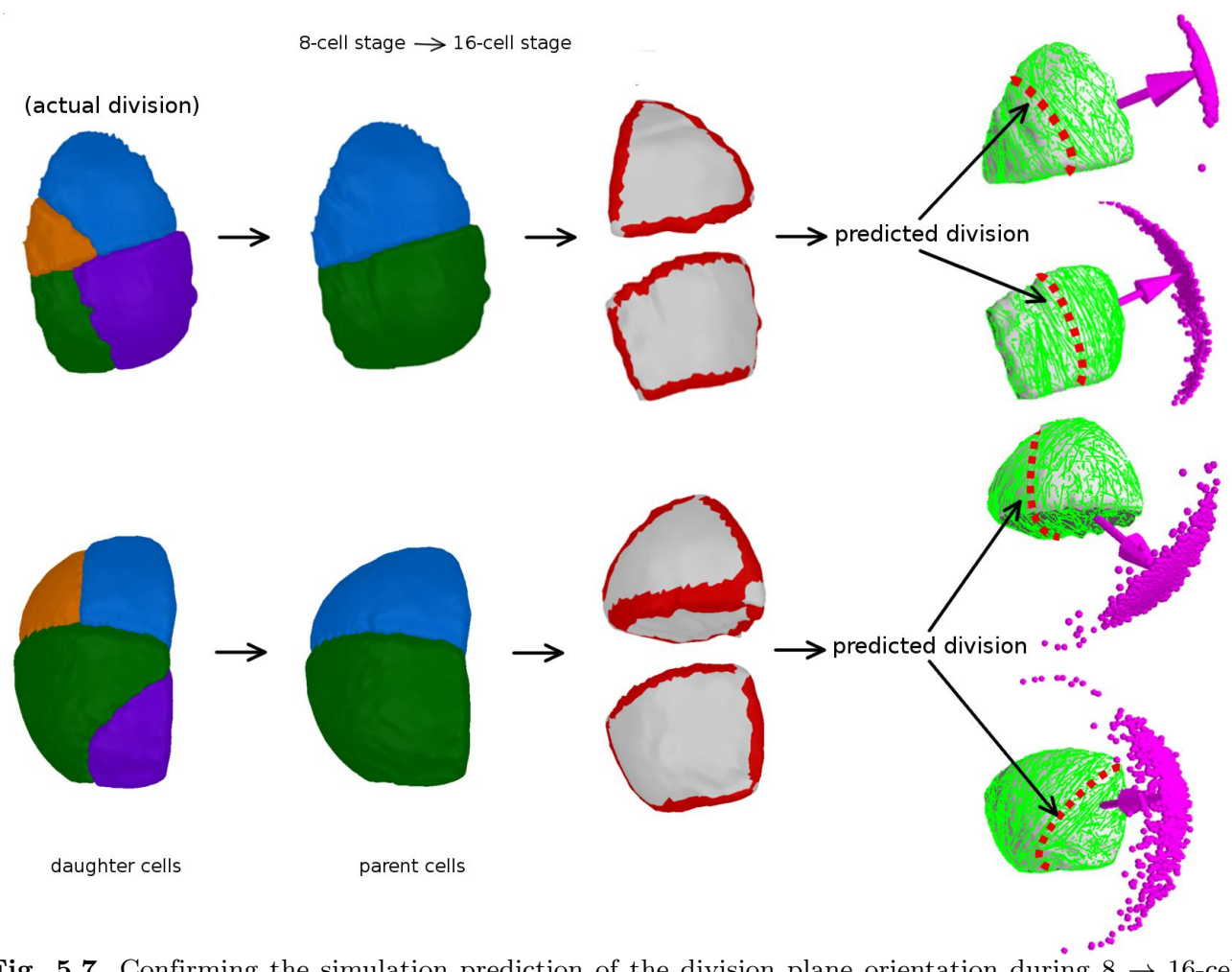

Fig. 5.7. Confirming the simulation prediction of the division plane orientation during $8 \rightarrow 16$-cell stage transition on $b d l$ cell shapes of Arabidopsis. We took cell shapes from 16-cell stage and merge the corresponding daughter cells to recreate the dividing parent cells (reverting to 8-cell stage). We simulated microtubule dynamics on the recreated parent cell shapes (i.e the recreated 8-cell stage) to get a prediction of their division plane orientation in the 16-cell stage. From the available experimental samples, we choose two different instances (more frequent in experimentally observed samples) of 16-cell stage for simulation. Simulated microtubule array orientation successfully predicted the expected division plane orientation. Microtubules were simulated with edge-catastrophe at the cell edges. Simulations were performed for $\approx 1000$ different configurations. Each dot point represents the tip of an orientation vector associated with a simulation configuration, and perpendicular to an associated microtubule array. The arrow vector is drawn through the mean position of all the dots in a cluster and represents the mean orientation of the associated microtubule array. Cell edges are coloured red to indicate that in simulation microtubules were subjected to edge-catastrophes. The red dotted line represents predicted average alignment direction of the associated microtubules, thus the division plane orientation. Cell templates used in these simulations were provided by Thijs de Zeeuw (WUR).

$(\approx 18 \%)$ and correct vertical $(\approx 82 \%)$ divisions were experimentally observed in the $b d l$ mutation. We designed toy simulations to identify the required factor to predict the exact ratio of horizontal to vertical divisions during $1 \rightarrow 2$-cell stage transition in $b d l$. Requirement of auxin response has already been reported for the vertical division that have been observed in wild type, but the exact mechanism for the contribution of microtubule dynamics is not yet known (Yoshida et al., 2014). We didn't find a significant difference in cell shape between the $b d l$ mutated and wild type 1-cell stage embryo, indicating that only cell shape and edge-catastrophe could not establish a vertical division. At this early stage of embryonic development, we didn't observe significant growth, removing the possibility of having the effect of cell growth on the dynamics of cortical microtubules. This 
suggests that to explain the auxin induced vertical division, in simulation a necessary factor needs to be incorporated in a way that recapitulates a corresponding vertical orientation of the microtubule array. In terms of shape attributes, the 1-cell stage is relatively simple, as it is composed of two cell-faces (a basal face shared with the suspensor cell and other approximately hemispherical non shared face) and one cell-edge that connects those two faces. The simplicity of this shape delimits the possibilities of factors, required to incorporate in the microtubule dynamics to obtain a vertical array orientation:

- Case I: Simulating microtubule dynamics without edge-catastrophe (default shape).

- Case II: Simulating microtubule dynamics without edge-catastrophe and enhanced stability ${ }^{(1)}$ to microtubules at the pole, i,e. a microtubule stabilizing apical polar cap.

- Case III: Simulating microtubule dynamics without edge-catastrophe and enhanced stabilization of microtubules at the basal face.

Simulation in study Case I (see Suppl Sec. S.1.1) and Case II (see Suppl Sec. S.1.4), resulted in random orientation of microtubule array, however Case III (see Suppl Sec. S.1.3) resulted in vertical orientation of the microtubule array, both in the $b d l$ and wild type cell shapes of 1-cell stage embryos. Based on these simulation results, we argued that the minimal assumption on the way by which the cell at 1-cell stage can establish a vertical orientation of microtubule array is to give enhanced microtubule stabilization at the basal face (shared with the suspensor cell), which is also the developmentally new cell face for this cell. Therefore, a possible mechanism of auxin effect is enhanced microtubule stability at the developmentally new cell face. In the $b d l$ mutation, a mixture of horizontal and vertical divisions suggested that auxin signalling may be partially affected at the 1-cell stage through a stabilizing mutation in the $B D L$ gene. In simulation, effect of the $b d l$ mutation on top of auxin signalling can be brought in the following ways:

- Case IV: Simulating microtubule dynamics with enhanced stabilization of microtubules at the basal face (auxin effect) and edgecatastrophe ( $b d l$ effect).

- Case V: Simulating microtubule dynamics with enhanced stabilization of microtubules at the basal face (auxin effect) and reduced edge-catastrophe, i.e lower probability of edge-catastrophe (partial $b d l$ effect).

${ }^{(1)}$ Enhanced face-stability corresponds to longer lifetime of the microtubules 


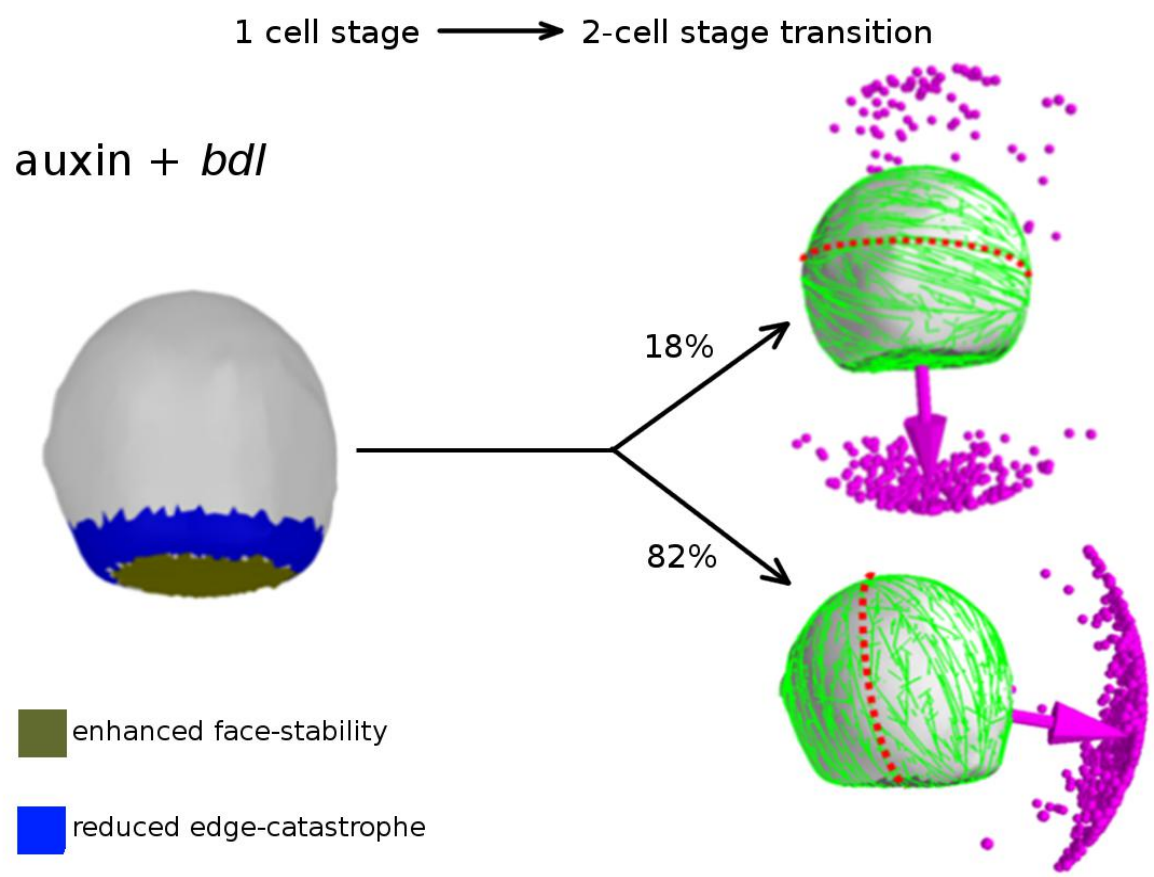

Fig. 5.8. Simulated orientation of microtubule array on embryonic cell shapes of Arabidopsis during $1 \rightarrow 2$-cell stage transitions, with enhanced stabilization of microtubules on developmentally newly inserted cell face and reduced edge-catastrophe in microtubule dynamics at the edge of that new face. For this particular case the developmentally new face is the basal face. Simulations were performed for $\approx 1000$ different configurations. Each dot point represents the tip of an orientation vector associated with a simulation configuration, and perpendicular to an associated microtubule array. Depending on choice of the strength of enhanced microtubule stability and reduction level of edge-catastrophe, we found two possibilities (two clusters of microtubule array orientation vector) microtubule array orientation. The arrow vector (in magenta colour) is drawn through the mean positions of all the dots in a cluster and represents the mean orientation of the associated microtubule array. In this particular simulation, we considered two fold enhancement of microtubule stabilization (i.e. two times reduction in microtubule dynamic catastrophe rate, $r_{c}$ ) at the new face and two fold reduction in edge-catastrophe (i.e two times reduction in microtubule edge-catastrophe multiplier, $E_{c a t}$ ) at the edge of the new face. The red dotted line represents predicted average alignment direction of the associated microtubules, thus the division plane orientation. Simulation was performed on cell shape that was imaged in the $b d l$ mutation. Cell template used in this simulation was provided by Thijs de Zeeuw (WUR).

Simulation in study Case IV (see Suppl Sec. S.1.2) resulted in only a mild fraction of vertical $(\approx 38 \%)$ and major fraction of horizontal $(\approx 62 \%)$ orientation of microtubule array, however study case Case V (see Fig. 5.8) with an appropriate ratio of enhanced stability and edge-catastrophe (reduced edge-catastrophe) to microtubules, predicted the experimentally observed fractions of vertical and horizontal orientations of division. In particular, a two fold enhancement of microtubule stabilization at the face of enhanced microtubule stabilization ${ }^{(2)}$ to microtubules at the new cell face (basal face) along with a two fold reduction of edge-catastrophe in microtubule dynamics, was able to generate the experimentally observed $\approx 18 \%$ horizontal and $\approx 82 \%$ vertical division in the $b d l$ mutation. In conclusion, we found that a

${ }^{(2)}$ Two fold enhancement of face-stability corresponds to two times reduction in the dynamic catastrophe rate of the microtubule at that face, which results in an over all increase in average life time of the microtubules at that face 
combination of the cell shape, cell edge-catastrophe and auxin mediated enhance stabilization of microtubules at the developmentally new cell face of the 1-cell stage embryo, were sufficient to give the cortical microtubule array orientation pattern, that follow the cell division in $b d l$ mutation.

\subsection{Anisotropic growth and/or stress may have role in division plane orientation}

The Arabidopsis zygote is highly asymmetric in shape and produces the first embryonic cell and a suspensor cell, through an asymmetric division. Like the zygote, the first suspensor cell is also highly asymmetric in shape and go through an asymmetric division. Among the resulting pair of daughter

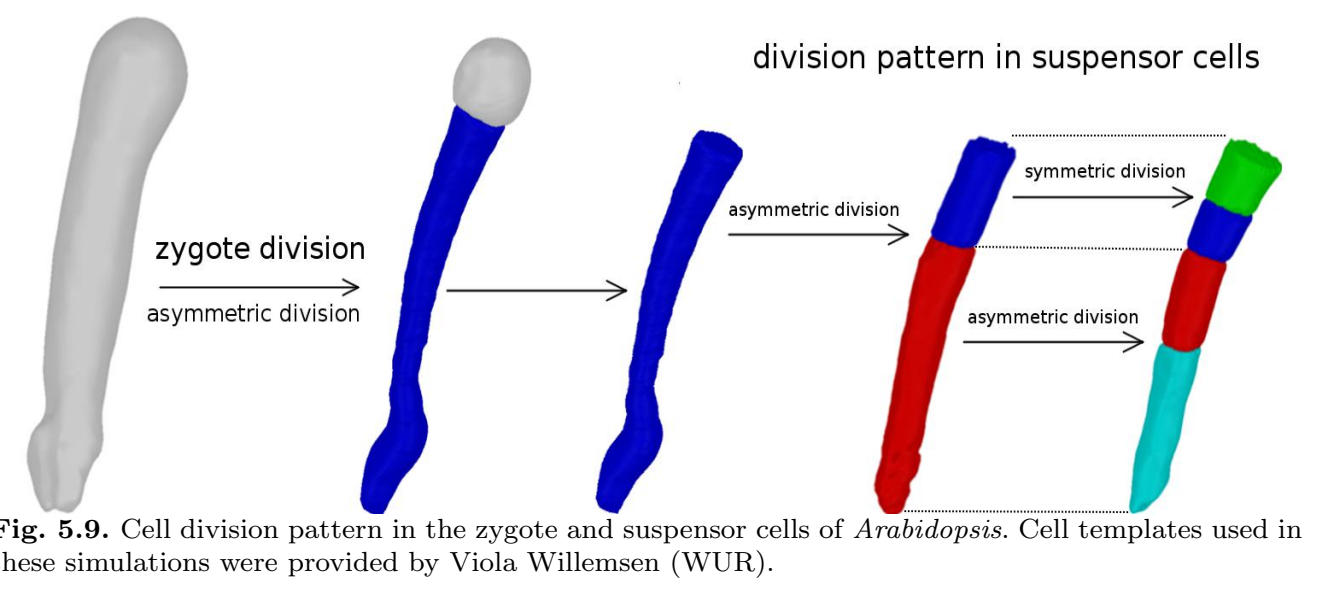

cells, the basal cell appears to be highly asymmetric and elongated in comparison to the upper cell. In the next division cycle, the asymmetric daughter at the basal further divides asymmetrically, while the upper one divides symmetrically (see Fig. 5.9).

\section{Division in zygote}

Being the first embryonic cell, the zygote does not contain any previous division plane, resulting in the absence of cell edges and edge-catastrophe in microtubule dynamics. Accordingly, we simulated microtubule dynamics on the surface of the zygotic cell shape without edge-catastrophe in microtubule dynamics. Our simulation revealed two preferred microtubule array orientations (see Fig. 5.10). The major fraction of the orientations predicted a horizontal division, which matched with the experimentally observed division plane orientation of the zygotic division, both in wild type and in $b d l$ mutation. However, a minor faction of the microtubule array orientation predicted a vertical division. Hence, our simulation result suggested the need 

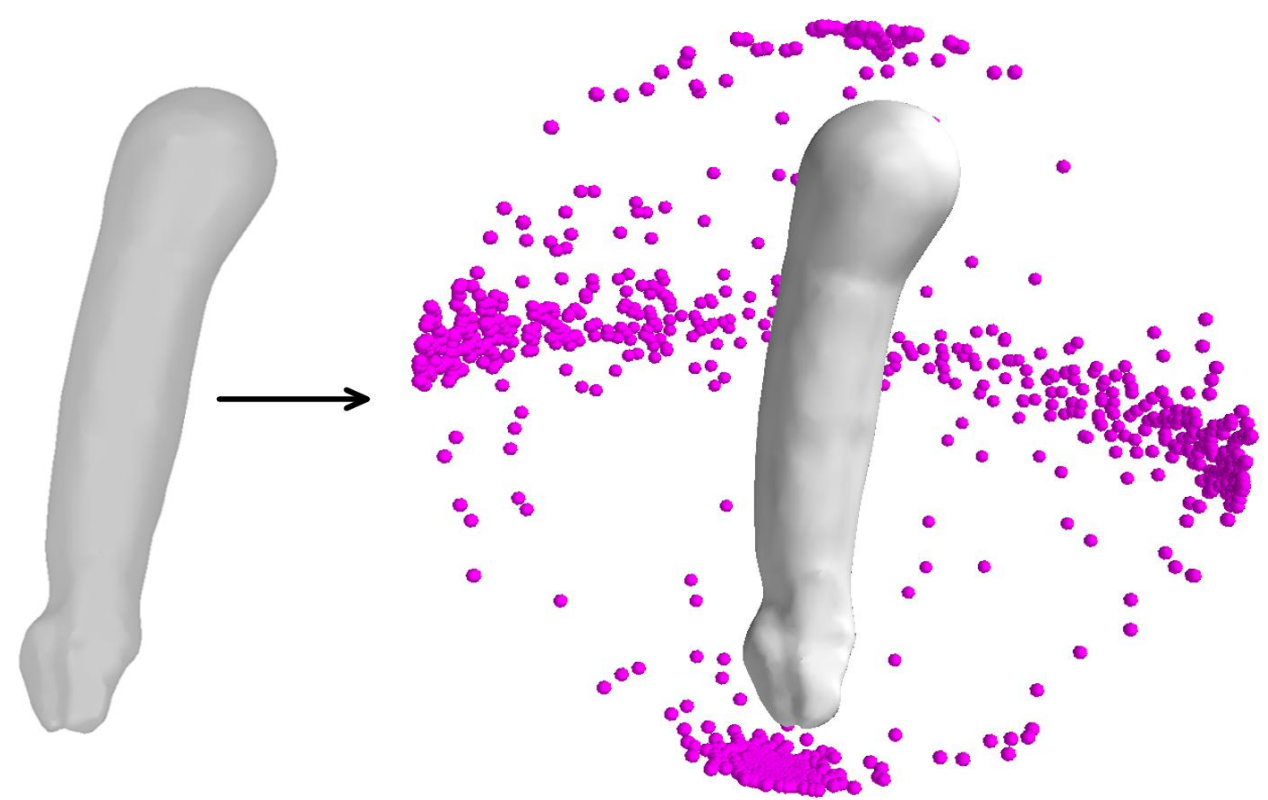

Fig. 5.10. Simulated orientation of microtubule array on the zygote cell shape of Arabidopsis. Simulations were performed for $\approx 1000$ different configurations. Each dot point represents the tip of an orientation vector associated with a simulation configuration, and perpendicular to an associated microtubule array. The cluster of dots that are $180^{\circ}$ away from each other represents same orientation of the associated microtubule array, hence considered as one cluster. We found two distinct clusters of microtubule array orientation. Simulation was performed on cell shape that was imaged under normal (wild type) condition. Cell template used in this simulation was provided by Viola Willemsen (WUR). Simulations performed on cell shapes that were imaged in the bdl mutation gave similar results.

for as yet unidentified additional factors other than auxin, to completely remove the existing possibility of vertical zygotic division. A possible hint towards this additional factor may be anisotropic growth, as it has been reported that the gene $Y O D A(Y D A)$ is required for elongation of zygote (Lukowitz et al., 2004). In loss of function mutants, i.e. yda, the zygote does not elongate properly though manages to divide horizontally (wild type division orientation). In contrast to wild type suspensor cell which divides horizontally, in $y d a$ the division is often vertical and with high frequency.

\section{Division in suspensor cells}

For the suspensor cells, the upper daughter contains two faces, and each of them are associated with an edge, and the lower daughter contains one edge bearing face. For the upper daughter, out of the two edges, one edge is associated with the cell face shared with the 1-cell stage embryonic cell, and the other edge is associated with the only edge of the lower daughter. We simulated microtubule dynamics on the surface of the suspensor cell shapes, both with and without edge-catastrophe in microtubule dynamics. For both 


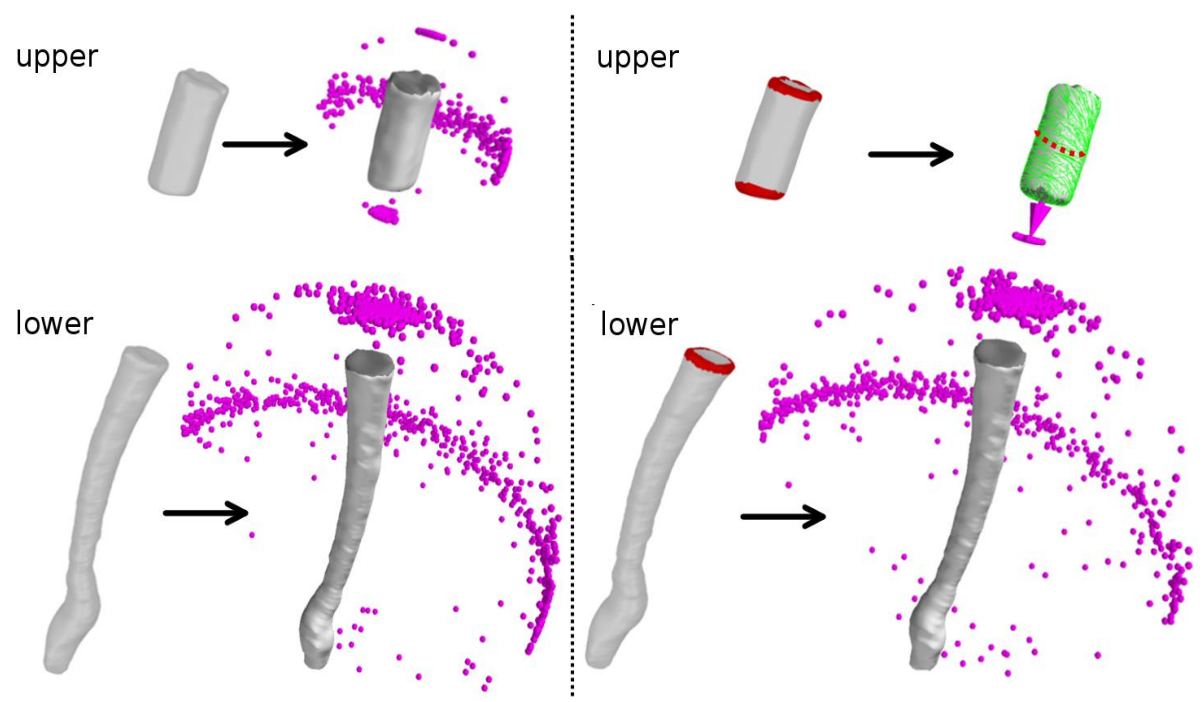

Fig. 5.11. Simulated orientation of microtubule array on suspensor cell shapes of Arabidopsis. Simulations were performed for $\approx 1000$ different configurations. Each dot point represents the tip of an orientation vector associated with a simulation configuration, and perpendicular to an associated microtubule array. The cluster of dots that are $180^{\circ}$ away from each other represents same orientation of the associated microtubule array, hence considered as one cluster. Independent of the inclusion of edge-catastrophe, the lower daughter (elongated) gave two possibilities of microtubule orientation (left/right, bottom panel). Inclusion of edge-catastrophe produces one unique orientation of microtubule array for the upper daughter (right, upper panel). Cell edges are coloured red to indicate that in simulation microtubules were subjected to edge-catastrophes. The red dotted line represents predicted average alignment direction of the associated microtubules, thus the division plane orientation. Here, simulations were performed on cell shapes that were imaged under normal (wild type) condition. Cell templates used in these simulations were provided by Viola Willemsen (WUR). Simulations performed on cell shapes that were imaged in the $b d l$ mutation gave similar results.

upper and lower suspensor cells, our simulations without edge-catastrophe in microtubule dynamics, revealed two possibilities of microtubule array orientations (see Fig. 5.11, left). Interestingly, our simulation with edgecatastrophe in microtubule dynamics, showed one unique possibility of microtubule array orientation for the upper daughter, but again (as without edge-catastrophe) the lower daughter produced two possible microtubule array orientations - horizontal and vertical (see Fig. 5.11, right). Presence of two cell edges within a close separation distance (due to the shorter longitudinal span) in the upper daughter, resulted in frequent edge-catastrophes along the longitudinal/vertical direction. As a consequence the microtubules preferred an alternative path of least edge-catastrophe, which is the horizontal direction. However, for the lower daughter, despite the edge, effectively the frequency of edge-catastrophe was negligible, due to the large longitudinal spanning of the cell. As a result, the lower daughter behaved almost like the default shape, i.e microtubule simulation without edge-catastrophe. Hence, independent of auxin signalling, the upper suspensor cells always divide horizontally. In the light of simulation result for the lower daughter, we inferred the necessity of an additional factor than auxin as in zygote, to exclude the vertical orientation of the microtubule array.

Considering the rapid elongation of the fertilized egg that form the 
basis of the shape anisotropy of the zygote, we surmised that anisotropic growth/stress might have an effect in the dynamics of microtubule, as postulated in the context of shoot apical meristem (Uyttewaal et al., 2012; Burian et al., 2013; Landrein and Hamant, 2013; Hamant et al., 2008; Sampathkumar et al., 2014; Heisler et al., 2010). For the lower suspensor daughter, there is no concrete evidence of rapid growth. It has been well studied that, for a give stiffness properties of the cell wall, shape anisotropy has the potential to generate anisotropic stress patterns, which have an impact on the dynamics of microtubule. This observation suggests that inhibition of anisotropic growth or a consequent change in stress anisotropy have a role in division plane orientation, i.e. on microtubule dynamics. The argument of stress anisotropy might also hold for the zygotic division. More generically, we think the growth anisotropy and/or shape anisotropy induced anisotropic stress patterns in the cell wall, might play a crucial role in oriented cell division by affecting microtubule dynamics (see Chapter 6) and the associated array orientation.

\subsection{Nuclear position correlates with the PPB formation site that determines location of division plane}

For $b d l$ embryonic development, the molecular basis behind the division patterns of the first five division cycle of zygote was understood via simulating microtubule dynamics of those cell shapes. Through simulated array of microtubules, we were able to predict the orientation of division plane but not the location of division plane, which plays a determinant role in symmetric/asymmetric divisions. In the context of early embryo development, instances of both symmetric and asymmetric divisions are observed. Particularly, the zygote and lower suspensor cells always divide asymmetrically, which requires to understand the process of specifying the division plane location, i.e the PPB formation. In plant cells, the preprophase band (PPB), a circular bundle of microtubules (along with actin filaments and associated proteins), predicts the insertion site of the new cell wall. It has been well studied that the formation of the PPB involve the dynamics of cortical microtubule and the formation of the associated microtubule array (Dhonukshe and Gadella, 2003; Hush et al., 1994; Vos et al., 2003). However, the mechanism behind the formation of the PPB is not yet understood. Among the many different conjectures, a compelling one is the "search and capture" mechanism of the formation of PPB (Vos et al., 2004). The core idea is that through increased dynamic instability, microtubules stochastically "search" for the domain of PPB in the cell cortex and get "captured" upon its encounter, via the cross-linking activity at the PPB domain. A small offset 


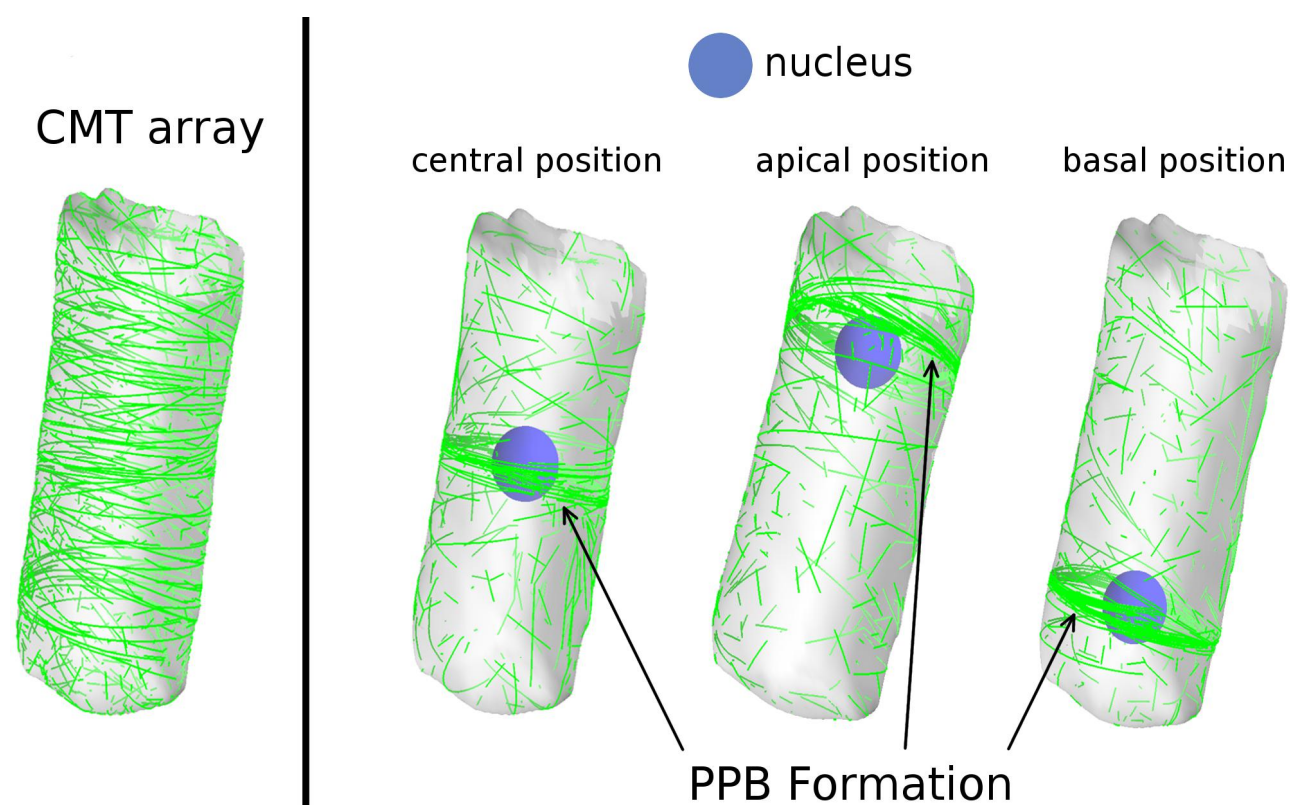

Fig. 5.12. Simulation of microtubules to understand the formation of PPB from an ordered (sparse) array of microtubule (left panel). We started from an ordered array of microtubules and initiated a small offset of increased nucleation rate at the region of the cell shape (cortex) that resides at the close vicinity of the nucleus. Within an hour (biological time) of triggering the offset, we observed the formation of a dense ring of microtubules, i.e the PPB. We performed simulations for three different positions (apical,basal and central; right panel) of the nucleus and observed a strong correlation between the nuclear position and the PPB location. Nucleus is shown as a sphere in light blue colour. The cell shape used in this simulation was provided by Viola Willemsen (WUR).

of few cross-linked microtubules, i.e due to microtubule nucleation from nearby nucleus of the cell in the PPB forming domain, was proposed to be sufficient to initiate the capture mechanism (Stoppin et al., 1994). Initiation of the capture mechanism at the close vicinity of the nucleus indicated a strong correlation between the nuclear position and the PPB location (Kimata et al., 2016). So, two parameters need to be considered to simulate the formation of PPB from an ordered (sparse) array of microtubule : (1) An increased rate of dynamic instability outside the PPB forming domain, and (2) small offset of increased nucleation rate at the close vicinity of the nucleus. From the corresponding experimental study, consideration of both parameters are necessary for the PPB formation. However, whether a single parameter of the two is sufficient or the relative contribution of these two parameters in the formation of the PPB is yet to be explored. Simulation has the advantage to efficiently address these different curiosities, which may be challenging in experiment. Accordingly, we simulated microtubules by using this minimal experimental evidence, to understand the formation of PPB from an initially sparse array of microtubules (see Fig. 5.12). In the experimental observation, the increased rate of dynamic instability outside the PPB forming domain, didn't lead to significant change in microtubule lengths. Our simulation output is characterised by a single input control parameter that depends only on the two lengths, namely average micro- 
tubule length (depends on the rate of dynamic instability) and microtubule interaction length (depends of nucleation rate). As the microtubule lengths outside the PPB forming domain didn't show a significant change during the PPB formation, we took this advantage and considered only a small increase of nucleation rate as the additional input variable. In the simulation, we started from an ordered array of microtubules and triggered a small offset of increased nucleation rate at the cell cortex that resides at the close vicinity of the nucleus ${ }^{(3)}$. Within an hour (biological time) of triggering the offset, indeed we observed the formation of a dense ring of microtubules from the initial sparse array. We varied the nuclear position and observed that the correlation was robustly maintained.

\subsection{Discussion}

In this chapter, we addressed the molecular basis of oriented cell division during early embryogenesis, particularly the first five division cycles of the zygote of the plant Arabidopsis when auxin signalling was compromised. Using a combination of experimental observation of division plane orientation and simulation of microtubule dynamics on realistic cell shapes, we explained the division patterns during the early embryonic development. We found that for the embryonic linage of cell divisions, which starts from the apical 1-cell stage embryo, a combination of cell shape anisotropy and edge-catastrophe in microtubule dynamics are sufficient to produce the array orientation patterns that predict the $b d l$ division plane orientation patterns. However, prediction of the asymmetric horizontal division of the zygote and the elongated lower suspensor cell, require additional factors, which we speculated to be the anisotropic growth/stress pattern that needs to be incorporated in the microtubule dynamics. Understanding the asymmetric division needs an approach of stepping out from just predicting the orientation of division plane and additionally a prediction of the location of division plane, i.e the preprophase band (PPB) domain. Our simulation study explains the strong correlation between the location of the PPB and nuclear position observed by experimental biologists, suggesting the existence of a mechanism of nuclear migration towards the PPB forming domain.

In the context of the first five division cycles of the zygote, the division plane orientation pattern observed in wild type (see Fig. 5.13) is different from the $b d l$ mutation, which compromised auxin signalling. Morphologically, the most significant difference in division plane orientation between $b d l$ and wild type have been observed during $8 \rightarrow 16$-cell stage transition. In contrast to the aberrant divisions in the $b d l$ mutation, in the wild type

${ }^{(3)}$ Simulation details of the PPB formation mechanism is described in Suppl Sec. S.2 
embryos, a precisely controlled division occurs that separates the inner and outer cells that create the protoderm. Based on this observation, it has been hypothesized that auxin response prevents a default shortest wall rule as defined by cell shape, forcing the cell to choose another division wall (Yoshida et al., 2014). Through our simulation approach, the correct

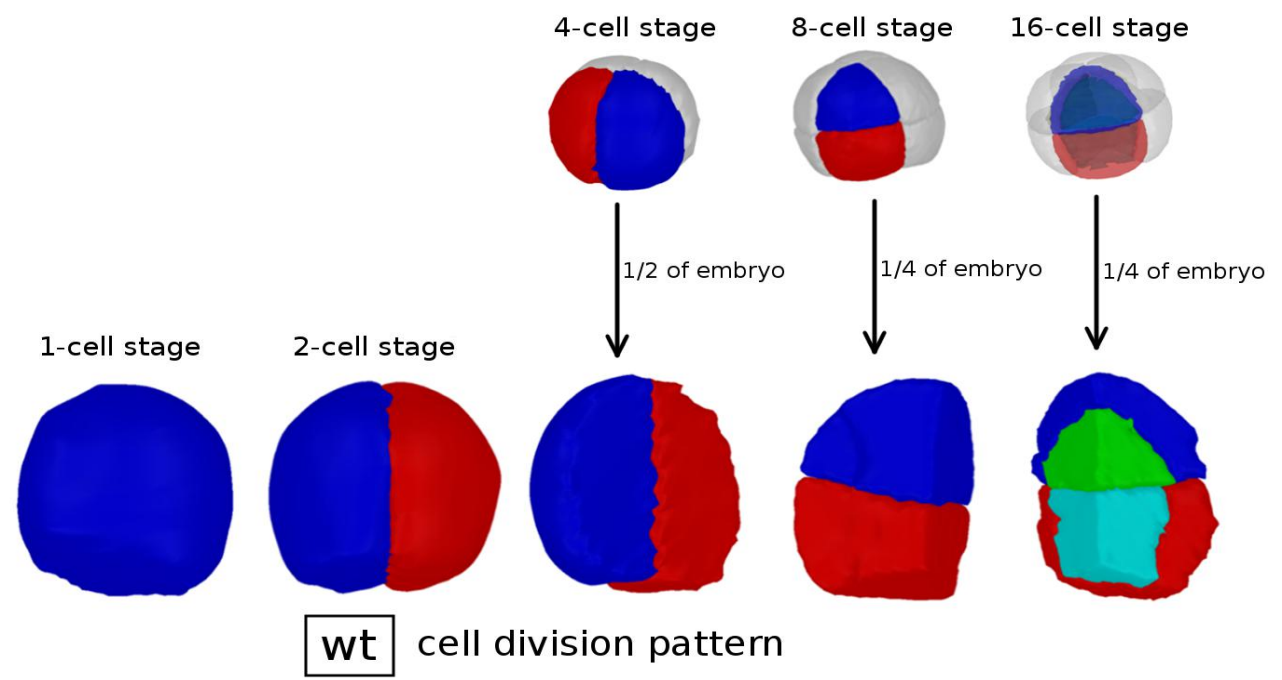

Fig. 5.13. Cell division pattern in wild type embryos of Arabidopsis. During $1 \rightarrow 2$-cell stage transition the cell division occurs vertically. The $2 \rightarrow 4$-cell stage transition shows vertical division. During $4 \rightarrow 8-$ cell stage transition, the division is horizontal. During the $8 \rightarrow 16$-cell stage transition, the division is parallel to the surface of the embryo. Cell templates used in this diagram was provided by Viola Willemsen (WUR).

prediction of division plane orientation in $b d l$ mutation by oriented array of microtubules and a simplistic explanation of asymmetric cell division, suggested a similar approach towards the molecular understanding of the wild type division pattern. Therefore, using our simulation understanding of the possible role of auxin in influencing the microtubule dynamics and the anisotropic growth/stress response of microtubules, we aim in future to study the wild type division pattern occurred during the early embryonic development. 


\section{Chapter 6}

\section{Division plane reorientation in Arabidopsis root epidermal cells}

In contrast to early embryogenesis, where division planes are chosen and cell divisions are executed without significant cell growth, the formation of organs involves cell divisions in growing tissues. For example, the Arabidopsis root has a main axis of growth with a group of dividing cells at the growing end of the root. These cells, collectively referred to as 'root
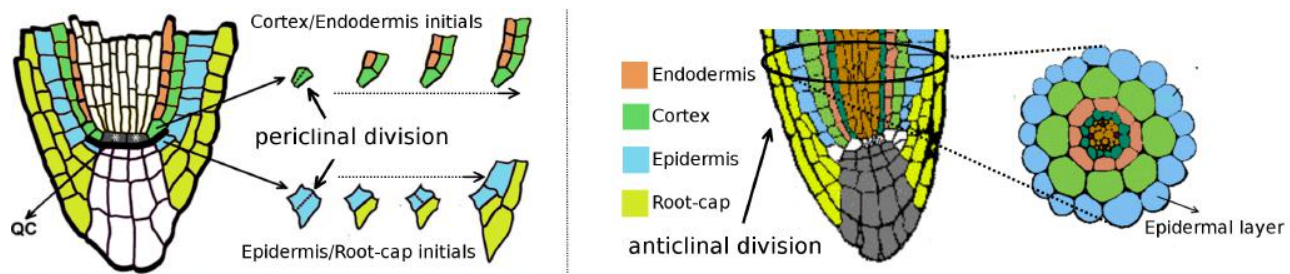

Fig. 6.1. Division plane orientations in Arabidopsis root cells: root initials/stem-cells (left panel) and differentiated root cells (right panel). Our experiments on differentiated root cells were focused on epidermal cells, so we also highlighted the epidermal layer (light blue, right panel). Images are adapted from Willemsen et al. (2008) and Dolan et al. (1993).

meristem', contribute new cells by divisions of which the majority is oriented perpendicular to the main axis of growth. In Arabidopsis and many other dicotyledonous plants, cells originate from distinct tiers of cells, referred to as 'closed meristem organisation' (vonGuttenberg, 1964). In many plants, the position of origin of these cell tiers contains slowly dividing cells called 'quiescent centre' (QC) cells. The cells at the end points of the cell files that make up each of these distinct cell layers, display division planes which give rise to one daughter cell occupying the position of its mother cell. These stem-cell like cells are referred to as initials: epidermis/root-cap initials, cortex/endodermis initials and columella initial (see Fig. 6.1, left). In Arabidopsis, all initials are in contact with the centrally positioned QC cells and these have been shown to be important to maintain the stem cell properties of the surrounding initials (van den Berg et al. 1997). The initials may display periclinal divisions, with a division plane parallel to the nearest root surface, thus creating new tissue layers. However, the cells born out of all the different initials that are positioned away from the centre develop into tissue specific cell types, and divide perpendicular to 
the principal growth direction of the root (Dolan et al., 1993), see Fig. 6.1, right). In this case the division planes are also perpendicular to the nearest root surface, and from classical definition, are called anticlinal divisions. As plant cells cannot move relative to each other, the entire root tissue architecture depends on properly oriented cell divisions.

The shape anisotropy of the root cells following from the growth anisotropy of the root, leads to pre-division cells with a longer dimension along the principal root growth direction and a shorter dimension in the plane perpendicular to the principal growth direction. Divisions perpendicular to the principle growth direction fit the classical division rule proposed by Hofmeister (1863), but also the postulate of a "shortest wall" rule of cell division (Dupuy et al., 2010; Smith et al., 2006; Stoma et al., 2008; Besson and Dumais, 2011) as a default rule for root cell division. Neither of these rules have a molecular mechanistic basis so far. Experimental observations on anisotropically growing cells reveal that the orientation of the division plane matches with the orientation of the cortical microtubule array (Wang and Huang, 2014). However, the molecular/physical basis behind the establishment of such order and orientation of the cortical microtubule array remained unclear. The possible known default factors that control the cortical microtubule dynamics are cell shapes (Gomez et al., 2016) and edge-catastrophe (Ambrose et al., 2011). Additional factors that might also be involved are auxin-dependent transcriptionally regulated enhanced face-stability/reduced edge-catastrophe to microtubules. Considering these factors into microtubule dynamics, we were able to explain the oriented cell divisions during early embryonic development (See Chapter 5). In the shoot apex, stress-related changes in cortical microtubule stability have been proposed to play a role in tissue growth (Uyttewaal et al., 2012; Burian et al., 2013; Landrein and Hamant, 2013; Hamant et al., 2008; Sampathkumar et al., 2014; Heisler et al., 2010). Here, we have analysed cell division orientations in the easily accessible Arabidopsis root epidermis in an attempt to combine microtubule network based cell division rules, that we have derived in mostly non-growing embryonic cells, with potential rules operating in growing cells inspired on the findings in the shoot apex.

It has been shown that the expression of the PLETHORA ${ }^{(1)}$ transcription factor strongly correlates with a transcriptional response maximum to the plant hormone auxin in the root tip (Aida et al., 2004; Xu et al., 2006). In the root, the PLTs are expressed in a gradient manner (see Fig. 6.2, right), where the expression level is highest in the meristematic zone and gradually decreases towards the elongation and differentiation zone (Galinha et al., 2007; Mähönen et al., 2014). In the stem cell region of the meristematic zone where the PLT level is highest, the epidermis/root-cap initials divide in a

\footnotetext{
(1) The PLETHORA1 (PLT1, At3g20840) and PLT2 (At1g51190) genes encode AP2domain transcription factor family members
} 

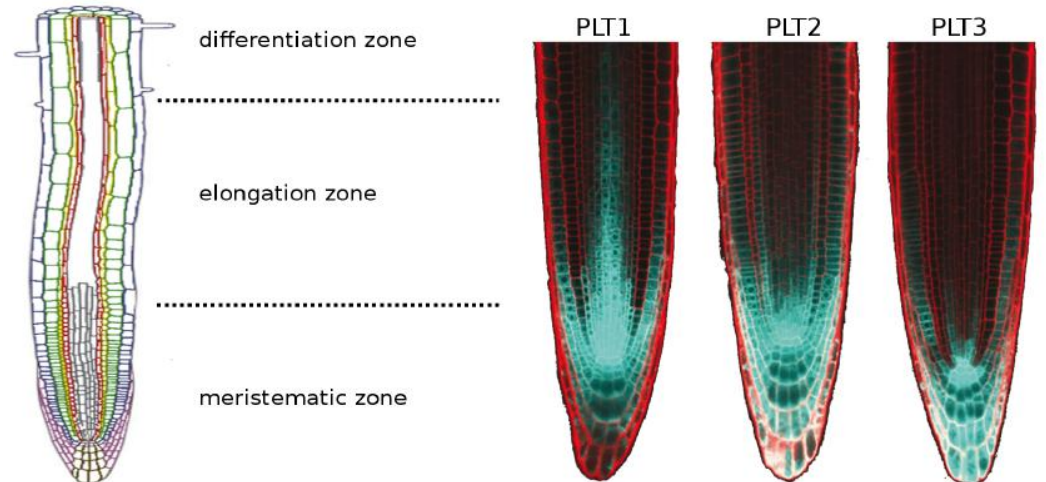

PLT expression

Fig. 6.2. Different growth zones in Arabidopsis root and expression pattern of the plethora transcription factor (PLT; PLT1,PLT2,PLT3). Images are adapted from Ubeda-Tomas et al. (2012) and Galinha et al. (2007).

periclinal orientation more frequently and grow very slowly. More mature epidermal cells encounter anticlinal divisions. Here we use induction of PLT expression as a means to change cell division orientation in the epidermis and analyse how this reorientation is affected by auxin signalling and edge catastrophe. Our recordings of precise cell shapes and volumes are then used in simulations of microtubule ordering, which reveal that, besides the rules derived from non-growing cells, an additional mechanism must be active to ensure proper orientation of cell divisions in the growing root.

\section{Distinguishing parent cell and just divided parent cell}

To trace the orientation of the division planes and extract the cell shapes for microtubule simulation, using MorphograpX, the experimentally obtained images were three dimensionally segmented. Tracing of division plane orien-
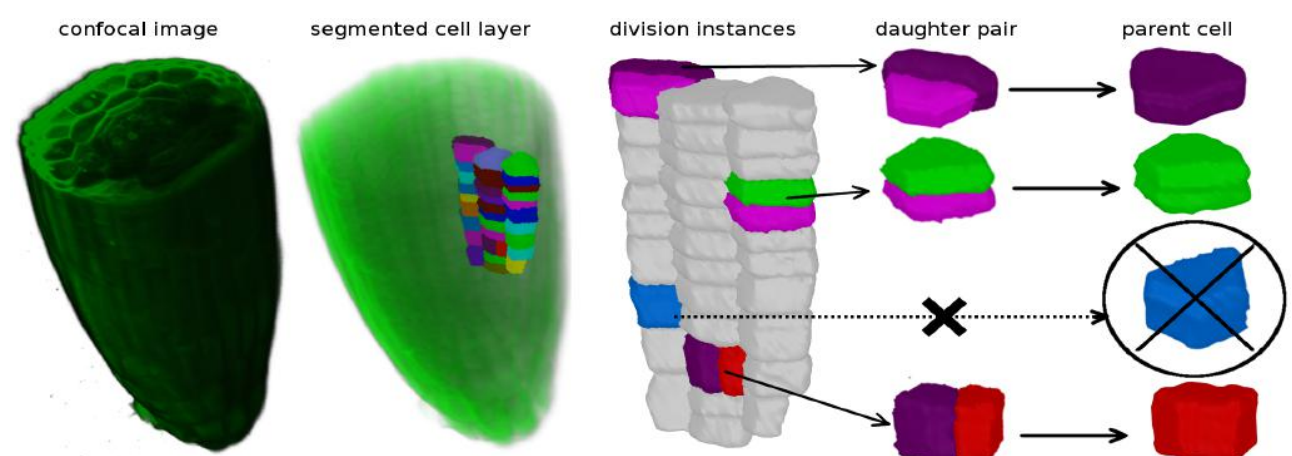

Fig. 6.3. Three dimensional segmentation of the confocal image of a Arabidopsis root to detect the daughter pair of just divided cells (segmented cell layer). A clear distinction between a non-divided parent cell and a just divided parent cell can be made by detecting the daughter pair of just divided cells (division instances; three different division instances are shown here) and non-divided parent cell (division instances; light blue). Microtubule simulation will be performed on the parent cells that will be re-created by merging their corresponding daughter pairs (daughter pair $\rightarrow$ parent cell). Non-divided cells (parent cell; cross marked) will be excluded from simulation. Image segmentation credit: Viola Willemsen (WUR).

tation was done by identifying the daughter pair of just divided parent cells (detailed procedure is illustrated Fig 6.3). To make sure that simulation of 
microtubule dynamics are performed on an about to divide cell cortex, we re-created the parent cell shape by merging the corresponding daughter pair. Non-divided cells were considered as still at the developmentally growing stage, thus ignored (see Fig. 6.3, cross marked).

\section{Classification of division plane orientations}

First, we quantified division plane orientations of epidermal cells and epidermal stem-cells, in wild type Arabidopsis roots and in mutants perturbing auxin regulation and edge catastrophe rates. In our experiments, we observed three different types of division plane orientation. To make a clear

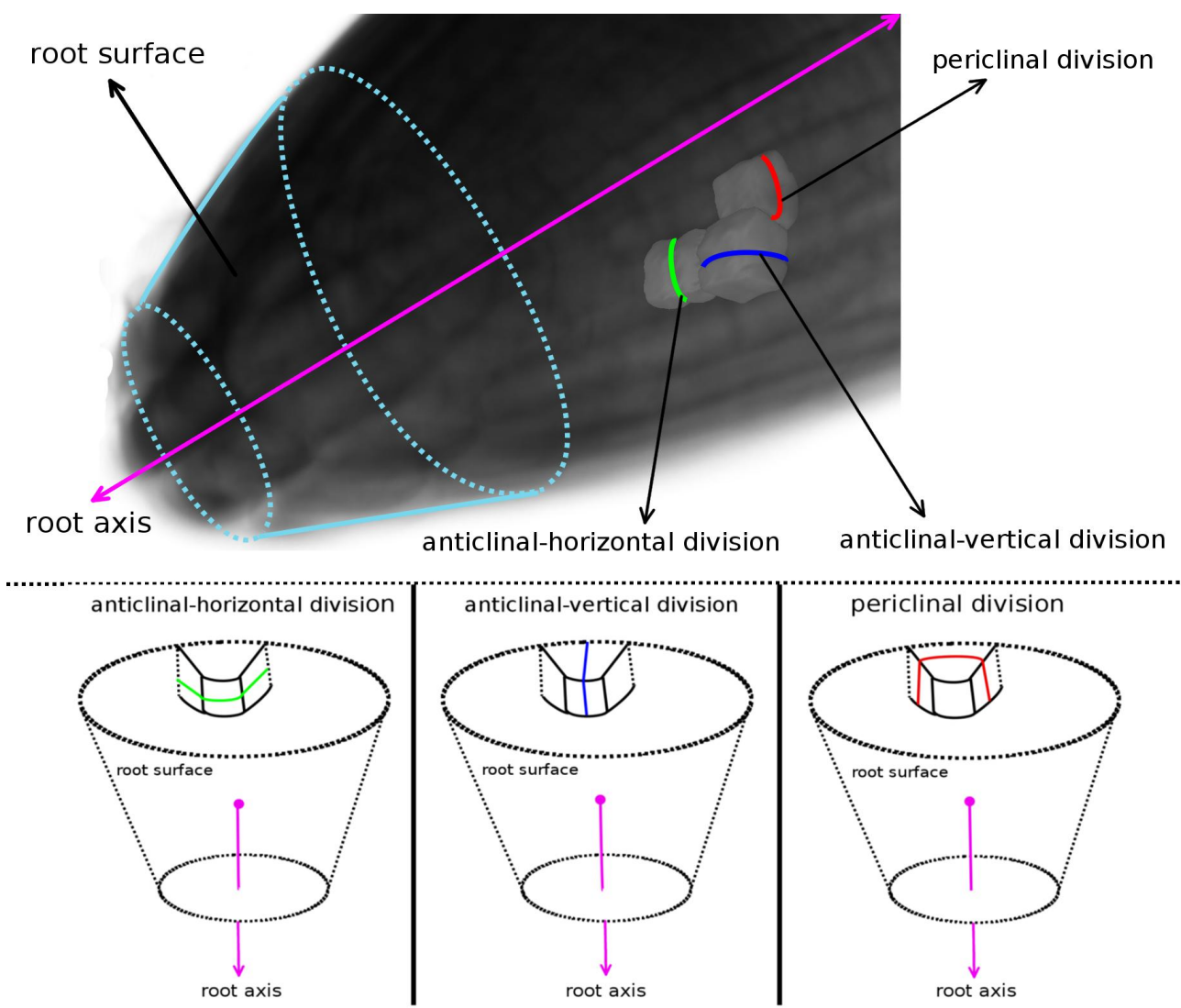

Fig. 6.4. Classification/re-definition of division plane orientations. A root axis is defined along the principle growth direction of the root (long arrow in magenta color) and the root surface follows the surface of the dotted cone like structure (cyan color). Three different types of division plane orientation are shown : anticlinal-horizontal (green color), anticlinal-vertical (blue color) and periclinal (red color), both within a root (upper panel) and schematically (lower panel).

distinction between the different types, we redefined the classical definition (i.e. anticlinal/periclinal divisions) of division plane orientations (see Fig. 6.4). We defined a root axis along the principle growth direction of the root. Then, we classified the different types of division planes with respect to the root surface and the root axis as,

- anticlinal-horizontal: The plane of division, that is at right angle to the nearest root surface and perpendicular to the root axis. 
- anticlinal-vertical: The plane of division, that is at right angle to the nearest root surface and parallel to the root axis.

- periclinal division: The plane of division, that is parallel to the nearest root surface.

\subsection{Divisions in normal condition}

In this experiment, 35S PLT2-GR plants were grown for three days in $\left(\frac{1}{2} \mathrm{~ms}\right.$ + dmso) medium. As a control experiment, they were transferred back into the same medium and were grown for another fifteen hours before imaging. Upon imaging, we observed that while the overall size of the epidermal cells

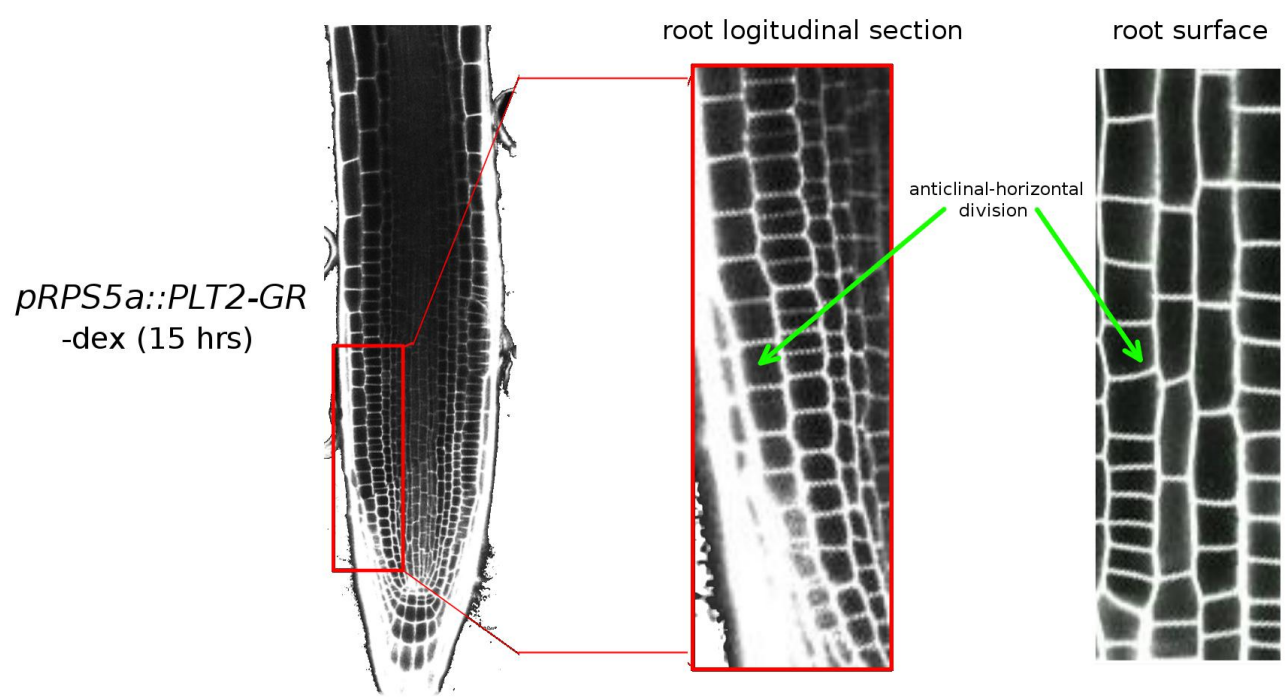

Fig. 6.5. Experimentally observed anticlinal-horizontal divisions in Arabidopsis root epidermal cells under normal (wild type) condition. The construct used in the experiment: PLT2-GR -dex (+15 hrs). Image courtesy: Viola Willemsen (WUR).

were different at the different root growth zones, they were able to retain a typical shape, which later we referred as WT-shape, independent of their position in the root. All the observed cell divisions were anticlinal-horizontal (see Fig. 6.5). We performed three dimensional segmentation of the roots using MorphograpX and extracted the segmented version of the epidermal cells (see Fig. 6.6, left), which were triangulated approximations of the actual cell surface (cortex). We scanned through those segmented epidermal cells which represented daughter pair of just divided parent cells (following procedure described in Fig.6.3), allowing us to trace the orientation of the corresponding division plane (see Fig. 6.6, right). For all such daughter pair of cells, we re-created the parent cells by merging their corresponding daughter pair into a single cell.To have a prediction of the experimentally 
observed division via the cortical microtubule array orientation, we simulated microtubule dynamics on the re-created (about to divide) parent cell shapes with edge-catastrophe in microtubule dynamics. Simulated microtubule array orientation predicted periclinal division (see Fig. 6.7), which contradicted the experimentally observed anticlinal-horizontal division. At
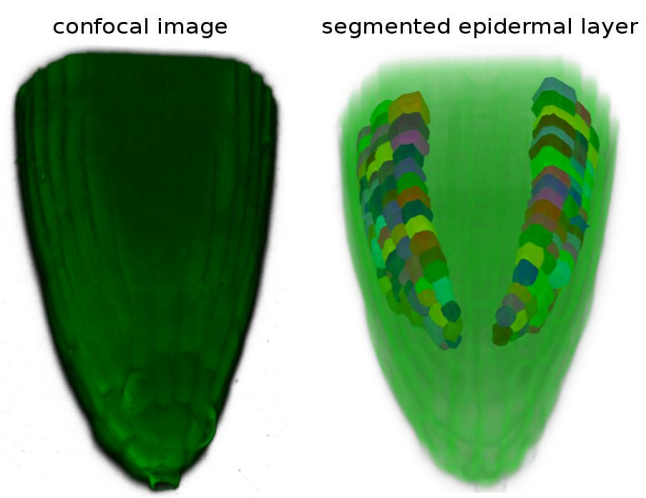

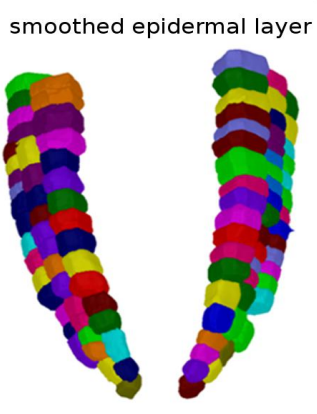

PLT2-GR -dex

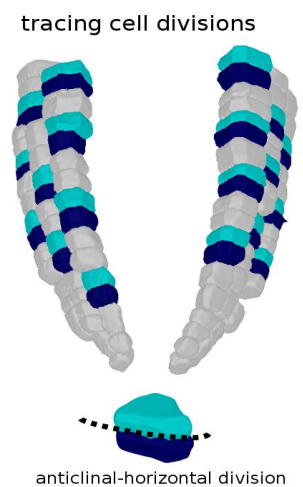

anticlinal-horizontal division

Fig. 6.6. Three dimensional segmentation of an Arabidopsis root grown under normal (wind type/PLT2-GR -dex) condition, for quantifying the division plane orientations of the epidermal cells (left panel). Tracing of all the divisions that took place in the epidermal layer of the sample (right panel). We observed presence of anticlinal-horizontal divisions only. The segmented cell shapes were used for microtubule simulation. Image segmentation credit: Viola Willemsen (WUR).

first sight this contradiction appeared quite striking, as we thought the experimentally observed anticlinal-horizontal division to be the default choice

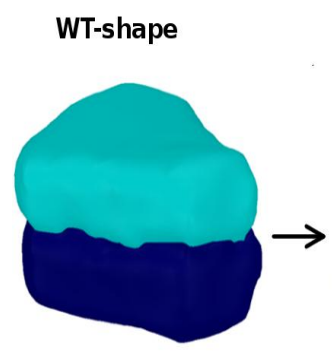

daughter cells

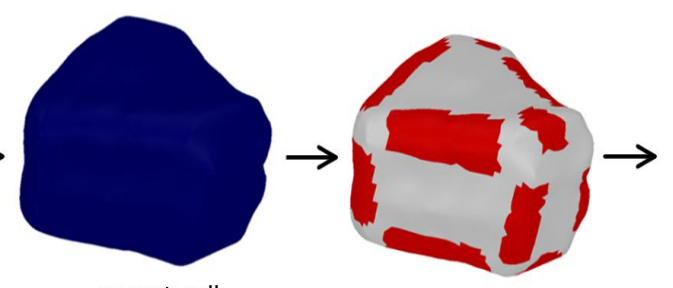

parent cell

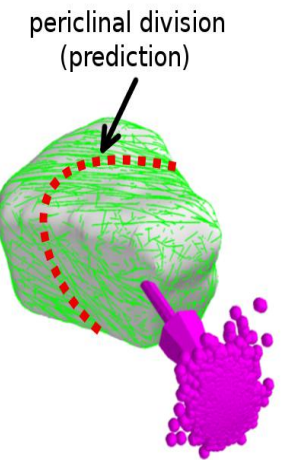

periclinal division

Fig. 6.7. Predicting division plane orientation in wild type Arabidopsis root epidermal cell shapes (WT-shape) through microtubule array formation. A parent cell was created by merging the corresponding daughter pair cells, obtained through the three dimensional segmentation of the root cells. Dynamics of microtubule was simulated on the recreated parent cell shape with edge-catastrophe in the microtubule dynamics at the cell edges. Simulations were performed for $\approx 1000$ different configurations. Each dot point represents the tip of an orientation vector associated with a simulation configuration, and perpendicular to an associated microtubule array. Cell edges are coloured red to indicate that in simulation, the microtubules were subjected to edge-catastrophes. The red dotted line represents predicted average alignment direction of the associated microtubules, thus the division plane orientation. Simulated microtubule array predicted periclinal division which was against the experimentally observed anticlinal-horizontal division.

of division for the epidermal cell, thus must be an outcome of cortical microtubule dynamics driven by cell shape anisotropy and cell edge-catastrophe. Simulation based on auxin rule, i.e developmentally new cell faces offer 
enhanced stabilization of microtubules, and the edges around these faces induce reduced amount of edge-catastrophe (auxin rules as derived from embryonic cell division, Chapter 5), did not help at all towards the rotation of the microtubule array to correct the orientation from periclinal to anticlinal-horizontal direction. This result suggests involvement of other factor that must overwrite the default/auxin based rules for microtubules to promote anticlinal-horizontal orientation of the microtubule array. We believe that the anisotropic growth of the root might be the factor. As such growth anisotropy can directly influence the microtubule dynamics by generating anisotropic stress, and also indirectly can create anisotropic stress pattern through generating shape anisotropy. Anisotropic stress can favour the orientation of microtubule array by forming bundles parallel to the direction of maximal stress (Landrein and Hamant, 2013).

\subsection{Divisions under PLT2 induction}

In Arabidopsis root the PLETHORA transcription factors (PLTs) are expressed in a gradient manner, with hight levels of expression in the meristematic zone. To identify the role of PLTs in division plane orientation,
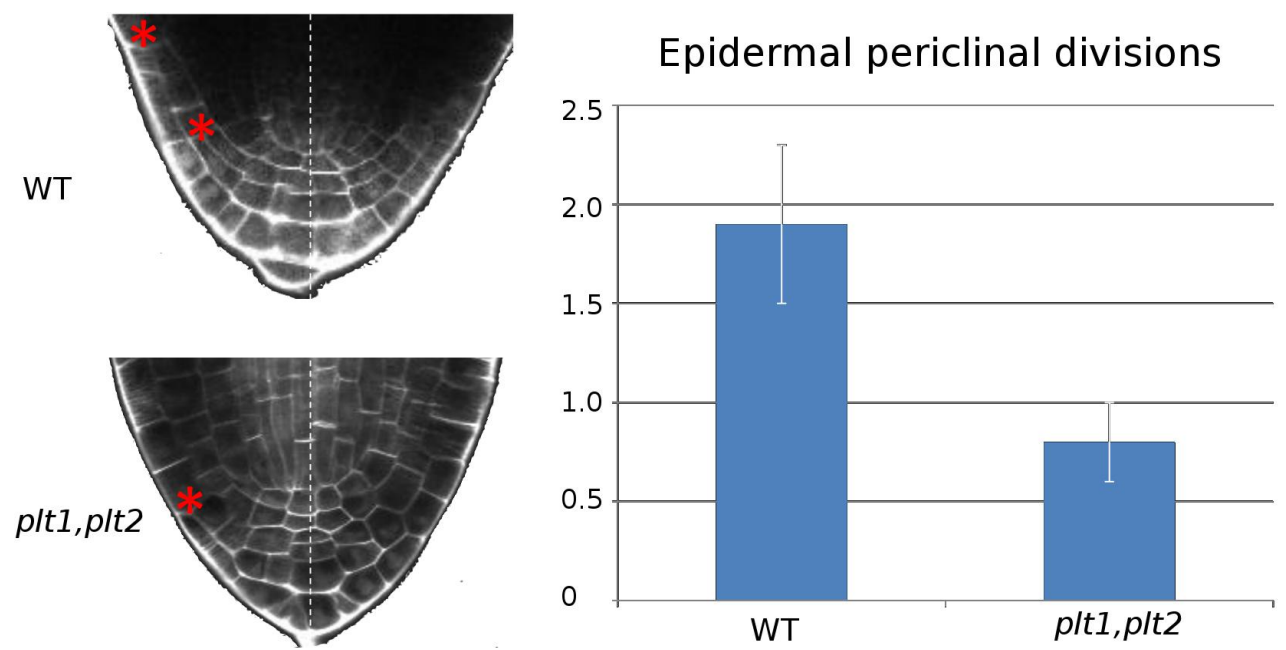

Fig. 6.8. Experimental comparison of periclinal division frequencies between normal (wild type/-dex) condition and under plt1,plt2 double mutant, for the cells in the Arabidopsis root meristematic zone. Use of the double mutant wipe out the PLT activities, resulting significant decrease in the periclinal division frequency. Image courtesy: Viola Willemsen (WUR).

we performed the PLT knock-out experiment and quantified the periclinal division frequencies in the root meristem zone. We observed a significant reduction in the frequency of periclinal division in PLT knock-out (plt1,plt2), when compared with the wild type (WT) condition (see Fig. 6.8). Our quantification of periclinal division was focussed in the meristematic zone, as the PLT genes are redundant in this zone, thus a knock-out in PLT-genes was expected to have stronger phenotype. Indeed, the outcome revealed active 


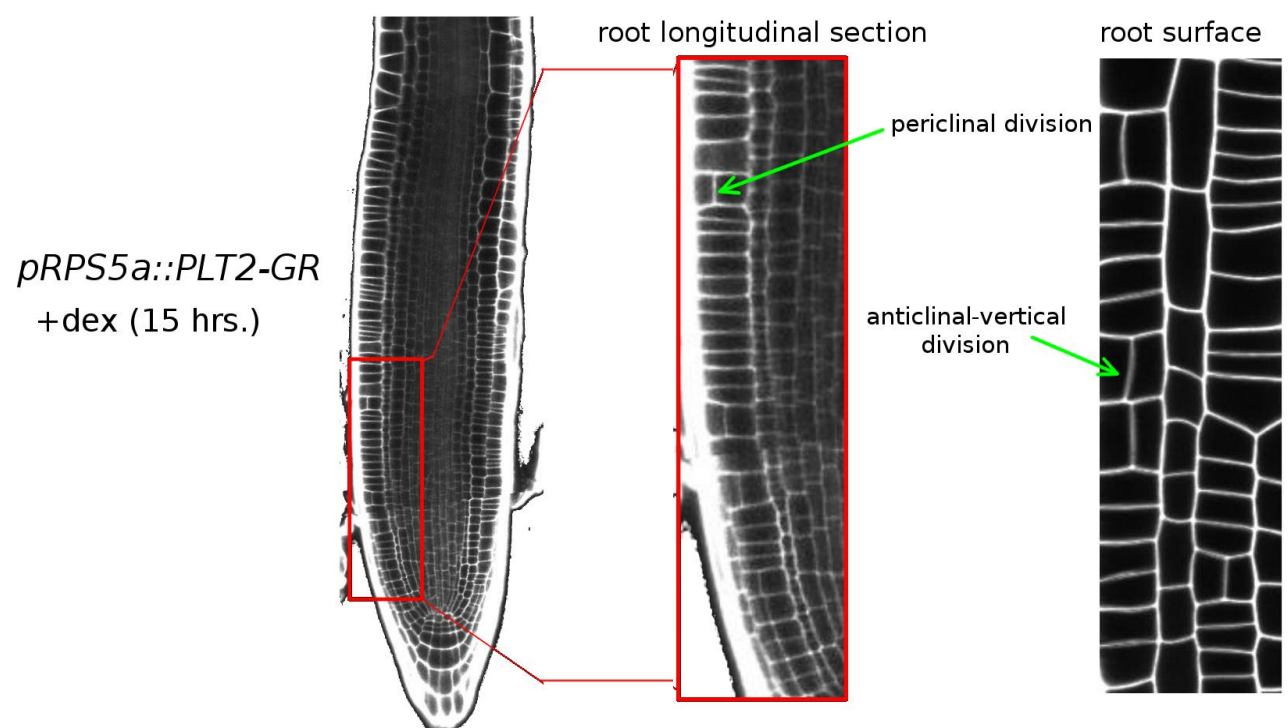

Fig. 6.9. Experimentally observed anticlinal-vertical/periclinal divisions in Arabidopsis root epidermal cells under PLT2 induction. The construct used in the experiment: PLT2-GR +dex (+15 hrs). Image courtesy: Viola Willemsen (WUR).

role of the PLTs in inducing periclinal divisions, which suggested that the over expression of PLTs in the PLT depreciated root zones might enhance the periclinal division frequency. Accordingly, we performed PLT2 overexpression experimentally in the root. In the experimental procedure, after growing 35S PLT2-GR plants for three days in $\left(\frac{1}{2} \mathrm{~ms}+\mathrm{dmso}\right)$, we transferred them into the $\left(\frac{1}{2} \mathrm{~ms}+\mathrm{dmso}+10 \mu M\right.$ dex $)$ medium and allowed to grow for another fifteen hours before imaging. The induction of dexamethasone (+dex) which activates PLT2, resulted in several instances of periclinal (see Fig. 6.9, root longitudinal view) and anticlinal-vertical (see Fig. 6.9, root surface view), Root longitudinal section view) divisions. We performed three dimensional segmentation of the roots using MorphograpX and extracted the segmented version of the epidermal cells (see Fig. 6.10, left), which were a triangulated approximation of the actual cell surface (cortex). We scanned through those segmented epidermal cells which represented daughter pair of just divided parent cells (following procedure described in Fig.6.3) that went through anticlinal-vertical/periclinal divisions. This allowed us to trace the orientation of the corresponding division plane (see Fig. 6.10, right). For all such daughter pair of cells, we re-created the parent cells by merging their corresponding daughter pair into a single cell. Looking at the re-created parent cells, we found that under PLT2 induction, the shape of the cells that encountered anticlinal-vertical/periclinal divisions, were significantly different from the WT-shape. From here on, among the cells that went through a change in shape and a consequent anticlinal-vertical/periclinal division under PLT2 induction, we referred the anticlinal-vertically diving cell shapes 

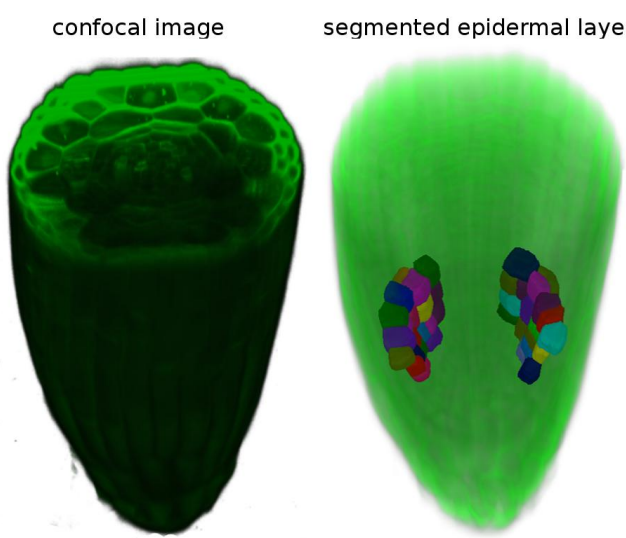

smoothed epidermal layer

Fig. 6.10. Three dimensional segmentation of an Arabidopsis root grown under the PLT2 induction (wind type/PLT2-GR + dex), for quantifying the division plane orientations of the epidermal cells (left panel). Tracing of all the divisions that took place in the epidermal layer of the sample (right panel). In addition to anticlinal-horizontal divisions, we also observed the presence of anticlinal-vertical and periclinal divisions. The segmented cell shapes were used for microtubule simulation. Image segmentation credit: Viola Willemsen (WUR).

as PLT-shape I and the periclinally dividing cell shape as PLT-shape II. We were curious to know whether the experimentally observed divisions can be predicted via simulated array of microtubules. Simulated microtubule array orientation with edge-catastrophe in microtubule dynamics at the cell edges, indeed gave correct predictions (see Fig. 6.11). A change in shape

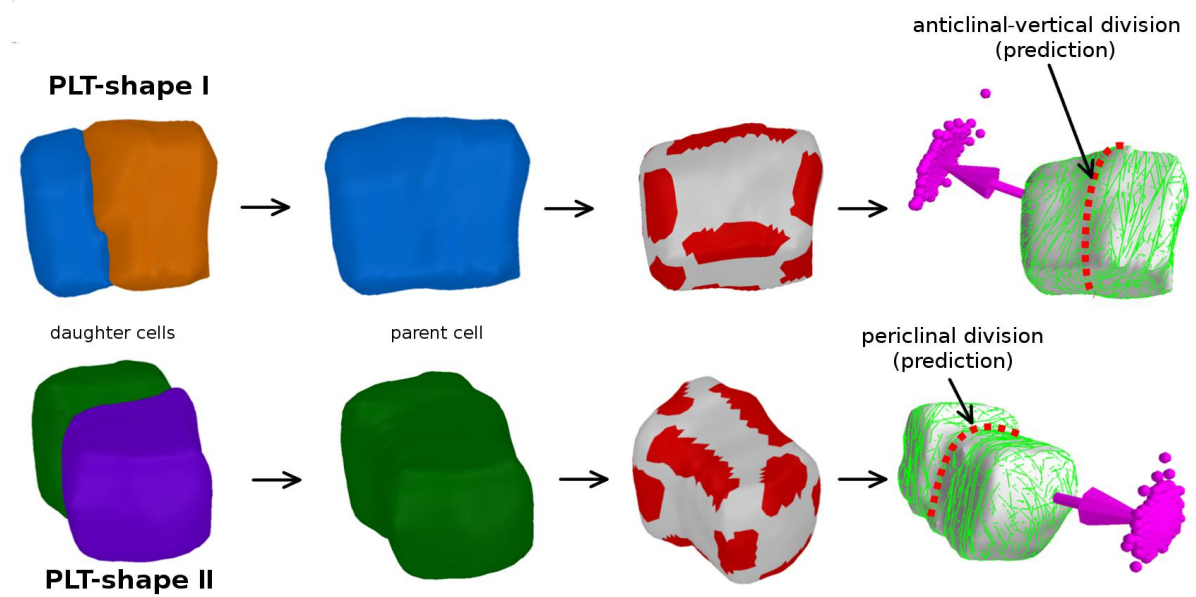

Fig. 6.11. Predicting division plane orientation in PLT2 induced Arabidopsis root epidermal cell shapes (PLT-shape I and PLT-shape II) through microtubule array formation. Parent cells were created by merging the corresponding daughter pair cells, obtained through the three dimensional segmentation of the root cells. Dynamics of microtubules was simulated on the recreated parent cell shapes with edgecatastrophe in the microtubule dynamics at the cell edges. For a given shape, simulations were performed for $\approx 1000$ different configurations. Each dot point represents the tip of an orientation vector associated with a simulation configuration, and perpendicular to an associated microtubule array. Cell edges are coloured red to indicate that in simulation, the microtubules were subjected to edge-catastrophes. The red dotted line represents predicted average alignment direction of the associated microtubules, thus the division plane orientation. Simulated microtubule array predicted the experimentally observed division for both types of cell shape.

anisotropy of a cell requires a corresponding change in its stress anisotropy, 
i.e a change in the principle stress direction. Here we asked the question, if the observed anticlinal-vertical/periclinal division under PLT2 induction was:

1. An immediate consequence of a change in stress anisotropy.

2. A consequence of a change in cell shape that was due to a change in stress anisotropy of the cell.

To see whether it was an immediate effect of a change in stress anisotropy, we extensively searched for the cells that did not undergo a change in shape from WT-shape but still went though an anticlinal-vertical/periclinal division under PLT2 induction. Indeed, we found such divisions that actually resembled the simulated prediction of division for WT-shape (separate simulation not shown as it corresponds to the similar outcome of Fig.6.7). However, observed occurrences of periclinal divisions in such shape were few. This observations suggested the necessity of quantitative analysis on the frequencies of different types of division for each of the three different types of shape (see Sec. 6.4). For PLT-shape I and PLT-shape II, it is very likely that in terms of microtubule response, these cells survived the immediate effect of the change in stress anisotropy, but their shape were affected. As a consequence, their division fate fell under the control of the modified cell shape and edge-catastrophe modulated microtubule dynamics. Division plane orientations predicted by the simulated orientation of the microtubule array in all the three different types of cell shape (WT-shape, PLTshape I and PLT-shape II) correspond to the divisions observed under PLT2 induction. Here, the prediction of division plane orientation through microtubule array orientation were made, by controlling the microtubule dynamics through default factors, i.e. cell shape and edge-catastrophe. This suggests PLT2 induction leads to the default choice of cell division. To form an array that predicts the wild type division, i.e. anticlinal-horizontal, an additional factor must come into play to override the impact of the default factors in controlling the microtubule dynamics. We think, this yet to be discovered factor out competed the effect of PLT2 induction in the cells, that continued to retain the WT-shape and anticlinal-horizontal division under PLT2 induction.

\subsection{Divisions in stem-cells}

In all our experimental root samples, independent of PLT2 induction (PLT2GR +/- dex treatment), the epidermal initial cell (epidermal stem-cell) always divided periclinally (experimental data not shown here) helping to form the lateral root cap and the epidermal layer. However, other 
experimental studies have shown other possibilities of the epidermal stemcell divisions as well (Campilho et al., 2006). In order to get a prediction of the division plane orientation through microtubule array formation, we simulated microtubule dynamics on the surface of epidermal stem-cell shapes
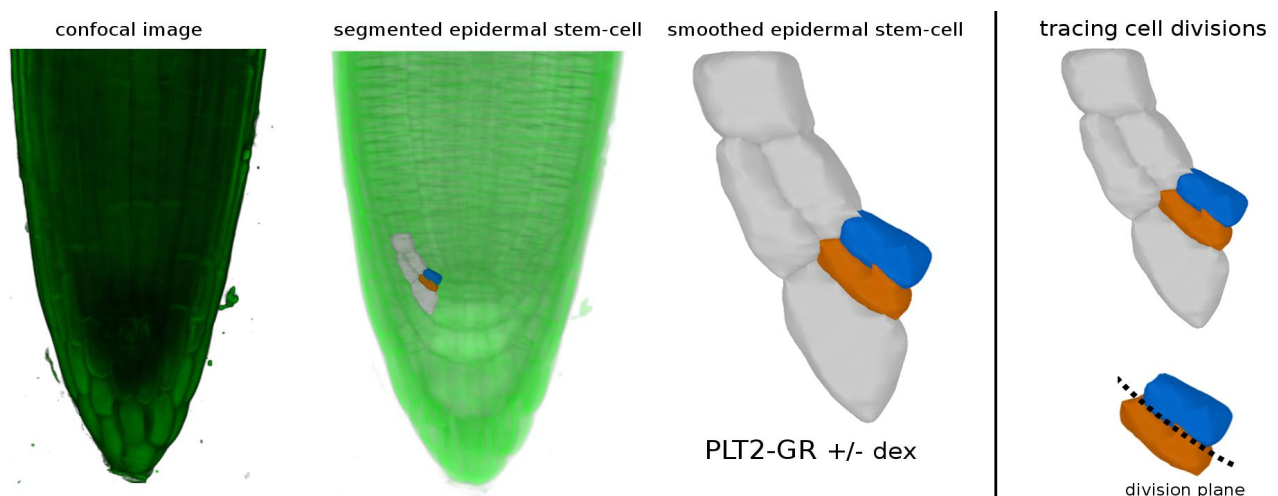

Fig. 6.12. Three dimensional segmentation of an Arabidopsis root grown under normal (wind type/PLT2-GR -dex) or PLT induced (PLT2-GR + dex) conditions, for quantifying the division plane orientations of the epidermal stem-cells (left panel). Tracing the divisions that took place in the epidermal stem-cells of the sample (right panel). We observed presence of periclinal divisions. However, occasionally an anticlinal-vertical divisions have also been observed. The segmented cell shapes were used for microtubule simulation. Image segmentation credit: Viola Willemsen (WUR).

(see Fig. 6.12, image segmentation process). As before, a dividing parent cell shape was generated by merging a daughter pair (epidermal/root-cap initials) of cells. The simulated orientation of the microtubule array with equal edge-catastrophe at the edges, predicted an orientation of the division plane, which was oriented somewhere in between anticlinal-vertical and periclinal division (hence undefined in Fig. 6.13). Here, we were inspired to reconsider the auxin based rule that we discovered in explaining the embryonic division plane pattern. Considering the auxin rule has a strong biological basis, as the epidermal initial cells are in the close vicinity of the stem cell niche where expression level of auxin is high. Accordingly, we simulated microtubule dynamics with the idea that newly inserted cell faces offer more microtubule stabilization, and compared to the old cell edges, the edges associated with the new cell faces offer reduced edge-catastrophe (auxin rule as derived from embryonic cell division, Chapter5). Interestingly with this rule, the simulated microtubule array orientation predicted the possibilities of both periclinal (confirming our experimental observation) and anticlinal-vertical divisions (Campilho et al., 2006), where the ratio of these probabilities can be varied by changing the strength of microtubule stability (edge-catastrophe) at the developmentally new cell faces (edges). Finding the molecular basis (through experiments) that offers such selective enhancement of stability or reduced edge-catastrophe to microtubules in auxin response, will be interesting. 


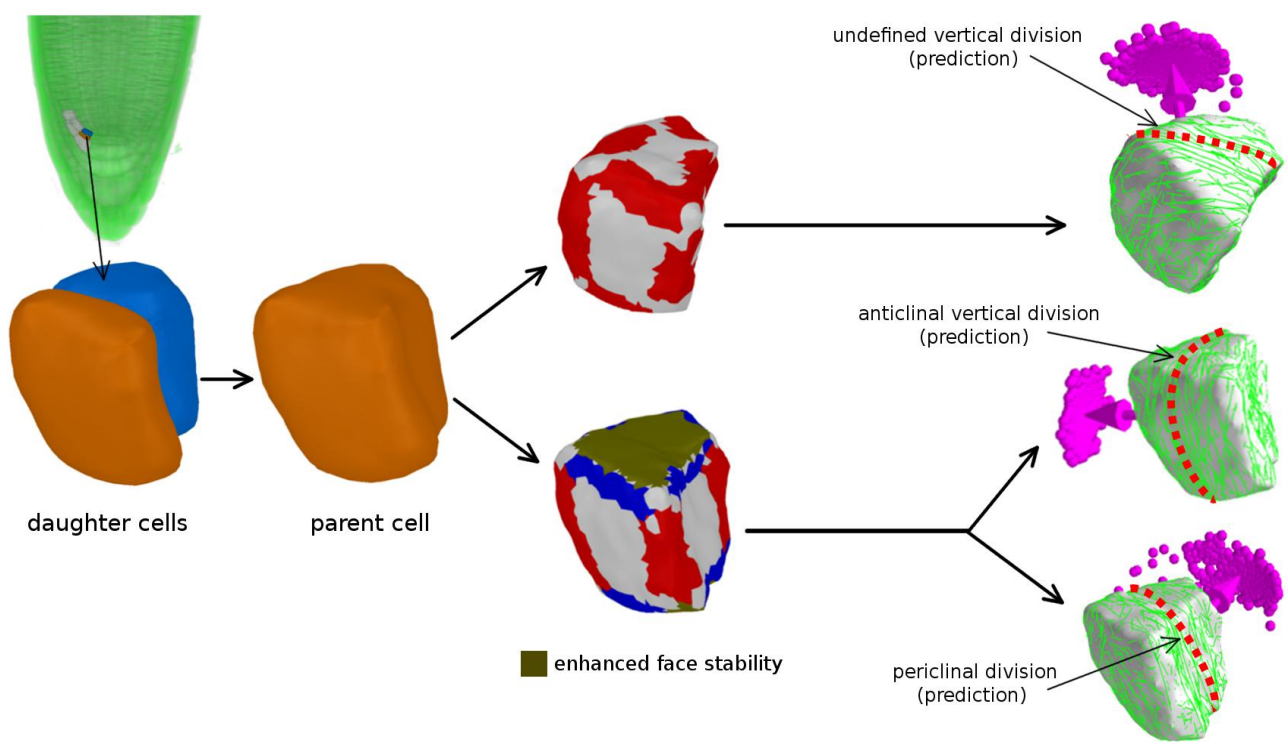

Fig. 6.13. Predicting division plane orientation in Arabidopsis root epidermal stem-cell shapes through microtubule array formation. A parent cell was created by merging the corresponding daughter pair cells, obtained through the three dimensional segmentation of the root cells (as shown in Fig. 6.12). For a given shape, simulation was performed for $\approx 1000$ different configurations. Each dot point represents the tip of an orientation vector associated with a simulation configuration, and perpendicular to an associated microtubule array. Dynamics of microtubule was simulated on the parent cell shape: (a) with edge-catastrophe at the cell edges which predicted one (anticlinal-vertical) of two possibilities of experimentally observed divisions, (b) with enhanced stabilization of microtubules at newly inserted cell faces and a corresponding reduction of edge-catastrophe at the edges of those new faces, i.e auxin rule. In this later case the simulated microtubule array predicted both possibilities (anticlinal-vertical/periclinal) of the experimentally observed divisions. Cell edges are coloured: (1) Red to indicate that in simulation, the microtubules were subjected to full edge-catastrophes and (2) Blue to indicate that in simulation, the microtubules were subjected to a reduced amount of edge-catastrophes. The red dotted line represents predicted average alignment direction of the associated microtubules, thus the division plane orientation.

\subsection{Quantitative analysis of division planes}

For better understanding of the biological process that leads to flip in division plane orientation or change in cell shape in the epidermal cell, we have decided to take a more quantitative approach. In this approach, our main goal is to quantify the root zone based frequencies of the different types of division, i.e. anticlinal-horizontal, anticlinal-vertical and periclinal divisions, that occur in wild type or PLT2 induction. This will give us an idea about the spatial correlation, if any, between the PLT2 expression and division plane orientation. To understand the role of PLT2 in changing growth/stress anisotropy of the epidermal cells, or the importance of growth anisotropy in the as yet to be understood wild type anticlinal-horizontal divisions, we want to make quantitative measure of the change in shape anisotropy between wild type and PLT2 induction. A computer program is under development in collaboration with the developers of MorphograpX, to specifically address these quantifications. Until now, the program can already quantify the orientation of the different types of division with respect to the root axis (i.e global orientation) and the three possible symmetric 

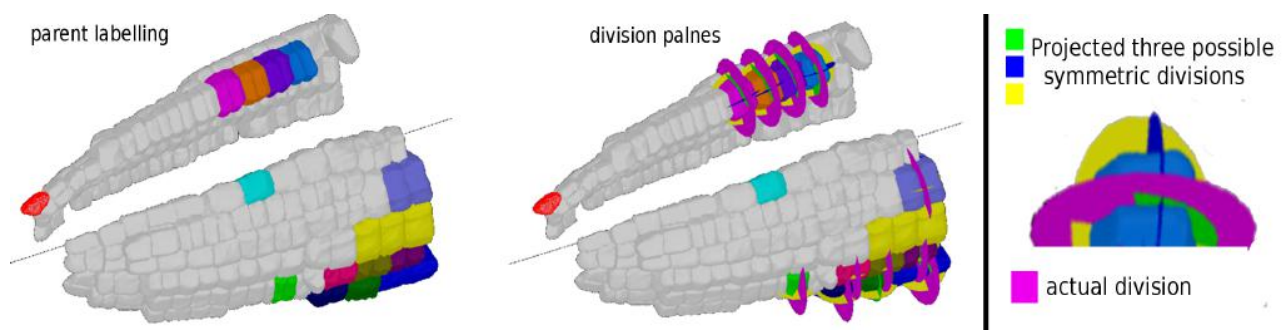

Fig. 6.14. Quantifying division plane orientations. A root axis was drawn through the center of the root and the root tip. The daughter pair of each divided cell were labelled with a unique index (parent labelling). A computer program was used to: (a) Find the closest approximation of the actual division plane (circular disc in magenta colour), and (b) Project the three best possible division plane (assuming symmetric division; circular disc in blue, green and yellow colour), perpendicular to the three principle axis of the cell. The orientation of the actual division plane was compared with respect to both the the root axis (global orientation) and the three possible symmetric division plane (local orientation). Credits:: Root segmentation: Viola Willemsen (WUR), Analysis tool: Soeren Strauss and Richard Smith (MPI).

divisions perpendicular to the three principle axis of the cells (i.e. local orientation). Briefly, in Fig. 6.14, we illustrate the procedure.

\subsection{Discussion}

Previously, we derived cortical microtubule array orientation rules in nongrowing early embryos and their correlation with cell division orientation. Here, using a combination of experiment and simulation, we looked for the mechanisms behind oriented cell division in anisotropically growing cell. We found that the experimentally observed anticlinal-horizontal division in the elongating root epidermal cell can not be predicted by the cortical microtubule array orientation, when the cell shape and edge-catastrophe were considered to be the only external modulator of microtubule ordering. This result suggested the presence of another factor in the formation of microtubule array in the epidermal cells. Under PLT2 induction, a large number of epidermal cells showed anticlinal-vertical/periclinal divisions and the shape of the cells differed from the WT-shape. Interestingly for PLT2 induced case, the simulated orientation of microtubules was able to predict the experimentally observed orientation of division plane. The change in the shape of the epidermal cells under PLT2 induction and consequently the match in microtubule array orientation with the division plane orientation, reconfirmed the role of shape anisotropy as an important factor in microtubule dynamics. However, the genetic pathway that links the PLT2 action with the cell shape deformation, needs to be explored. Also, finding the role of PLT2 in microtubule biochemistry that determines the orientation of microtubule array, hence division plane orientation, will be interesting. In general for anisotropically growing cells, it has been shown that the microtubule dynamics is influenced by the anisotropic stresses and the orientation of microtubules follows the maximum stress direction 


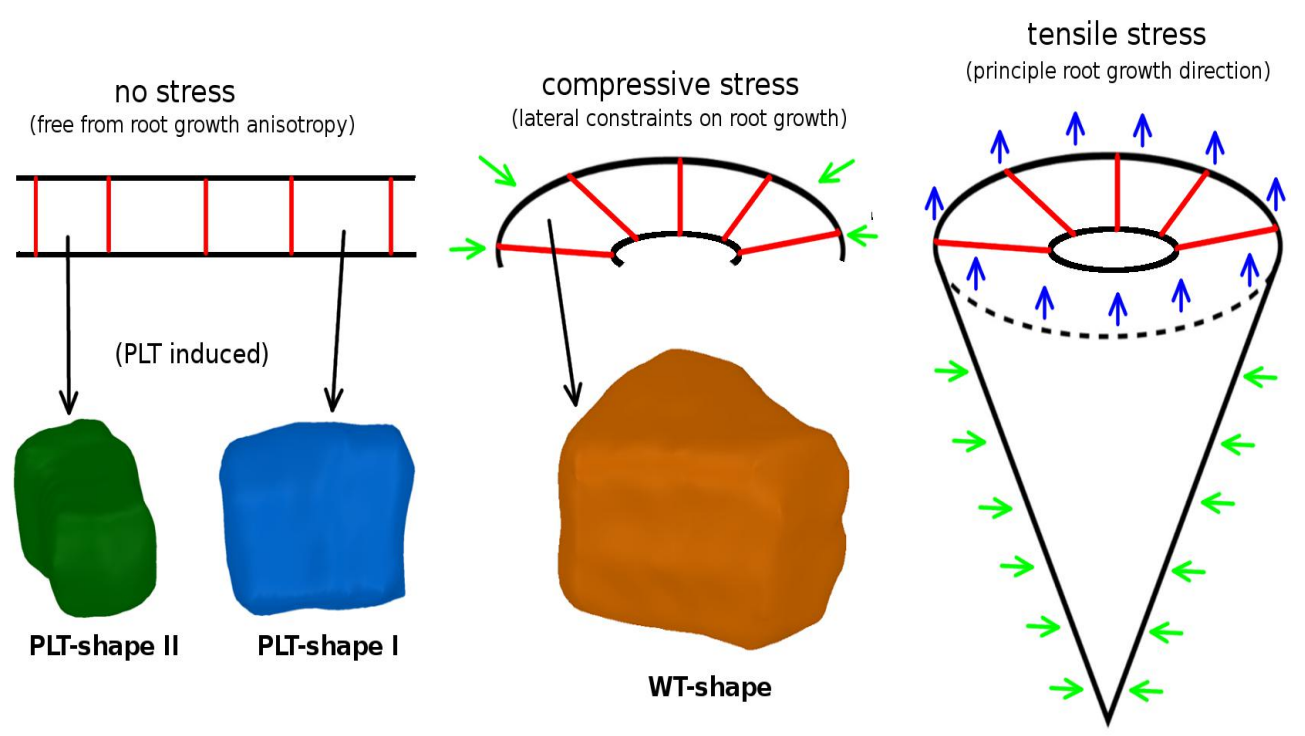

Fig. 6.15. Schematic view of the origin of anisotropic stress in root epidermal cells and the corresponding effect on cell shapes. An epidermal cell which would have the shape of a rectangular parallelepiped under no/low stress condition (PLT-shape I and PLT-shape II), will approximately take the shape of a round-edged (almost) pyramid (WT-shape) under the given compressive stress (green arrows) as shown above. Further due to the anisotropic growth of the root, an additional tensile stress (blue arrows) will be imposed on the cells. A competition between these two stress components will determine the principle stress direction on the cells, which further will influence the microtubule dynamics, as discussed in the text.

(Uyttewaal et al., 2012; Louveaux et al., 2016). A lot of effort has been put into understanding the relationship between the anisotropic cell growth and cortical microtubule array orientation (Baskin, 2001; Sugimoto et al., 2001; Himmelspach et al., 2003; Burk and Ye, 2001). A conceptual model on microtubule dynamics and their ability to self-organize in bundles parallel to the direction of maximal stress has also been proposed (Landrein and Hamant, 2013). In the proposed model microtubules were considered to have more catastrophes along the perpendicular direction of maximum stress, thus higher possibility of array formation along the maximum stress direction. The cells at the different stages of early development do not grow significantly which allowed us to neglect the growth effect in microtubule dynamics. In the context of root epidermis, an instance of slow growing cells are the epidermal stem-cells, which are located in the meristematic zone of the root where auxin level is high. These stem-cells divide periclinally with occasional instances of anticlinal-vertical division. Accordingly, the simulated orientation of the microtubule array with auxin based rules in microtubule dynamics, successfully predicted the possibilities of both anticlinal-vertical/periclinal divisions. However, presence of anisotropic growth in the root epidermal cells indicates consideration of the effect of the associated stress response to the microtubule dynamics. In Fig. 6.15, we present a schematic view of the origin of anisotropic stress in root epidermal cells and its effect of cell shapes. The WT-shape is likely a 
consequence of anisotropic stress. A relaxation in such stress anisotropy will lead to a different conformation of shape that closely relates to the shape that often arises due to PLT2 induction. Growth is rather a general property associated with any cell, independent of their specific type. From this point of view, out of our curiosity, we looked at the cortex cells as well. According to our expectations, while normal (wild type) cortex cells went through horizontal division, under PLT2 induction a significant number of cortex cells went through anticlinal-vertical/periclinal divisions (data not shown). This suggests, in the simulation model inclusion of cell growth effect on microtubule dynamics is important. Our modelling framework can be extended to simulate microtubule dynamics on growing cell, where the cell growth will be performed by using the Finite Element Method (Reddy, 2006). However, in the present situation, we can not incorporate the effect of cell growth in the dynamics of microtubule, as further experimental work is required to meaningfully bias (effect of cell growth) microtubule stability in a preferred direction (i.e. maximal stress direction). From the experimental side, it will be interesting to promote anisotropic cell growth inhibition, where expected outcome of such experiment will be a large number of anticlinal-vertical/periclinal division instead of the normal anticlinal-horizontal division. Based on existing genetic tools (Scheible et al., 2001; Harris et al., 2012; Worden et al., 2015), we can now perform such anisotropic growth inhibition experiments. 


\section{Chapter 7}

\section{General discussion and outlook}

Oriented cell divisions generate structured layers of cells that support growth in the right direction, which results in the efficient anisotropic growth that ultimately drives plant morphogenesis. Thus, understanding the mechanism of oriented cell division appeared to be a key factor towards the understanding of plant morphogenesis. The hitherto proposed rules of division plane orientation in plant cells are geometric rules, instead of rules underpinned by mechanisms based on cellular components involved in division plane orientation. Many experimental observations have shown that the orientation of division plane follows the orientation of the cortical microtubule array. In this thesis, we took a modelling approach that is inspired by experiments, to connect the molecular basis of oriented cell division via the cortical microtubule cytoskeleton to geometric rules. This chapter links relevant parts of the thesis, with an intention of addressing a broader question:

What is the relation between plant morphogenesis and the molecular basis of oriented cell division?

We succeeded in developing a modelling framework to investigate (simulate) the basic rules of microtubule dynamics. This framework allows us to integrate regulatory effects such as edge-catastrophe and the impact of shape anisotropy on microtubule dynamics (Chapter 2). A given cell shape by default includes basic microtubule regulators such as shape anisotropy and edge-catastrophe. A key control factor appears to be the minimum number of microtubule interactions, which suffices for the formation of a robustly ordered and orientated microtubule array. The modelling framework allows parametrization for robust order and orientation of the microtubule array through a control parameter as input, which can be set to yield the "ordered domain" of microtubules by tuning the number of microtubule interactions (Chapter 3). Furthermore, we defined a suitable output order parameter vector, which quantifies the order and orientation of the microtubule array. The modelling framework maintains its robustness against the inherent stochasticity in microtubule dynamics, such as spatial randomization in nucleation or occurrence of stochastic microtubule events. Such robustness can be realized via simulating different configurations with variable initial stochasticity, which lead to similar average orientation of the microtubule array. As the orientation of division plane follows the orientation of the 
cortical microtubule array, the same order parameter vector enables to predict the division plane orientation.

The robustness of the division plane orientation indicates buffering of the fluctuations that take place in the microtubule dynamic parameters due to different cellular processes. The parametrization of our simulation framework, which leads to the formation of robustly orientated microtubule array, meaningfully captures biological buffering of fluctuations in the different microtubule dynamic parameters.

Our simulation of microtubule dynamics on regular shapes turned out to be profoundly informative about the basic and regulatory effects on microtubule dynamics, that contribute to the formation of an ordered array (Chapter 4). We learnt that mutual interactions among microtubules are necessary to form an ordered array. Also, microtubules sense the shape anisotropy and accordingly form arrays only along selective paths that allow sufficient microtubule self-interactions. Additionally, microtubules avoid paths with a higher rate of edge-catastrophe and prefer a path of least edge-catastrophe to form an array. Our tests on the biological influence of different stabilization of microtubules at developmentally different cell faces indicated that enhanced stabilization of microtubules (microtubules have longer lifetime) at a certain face favours an array formation through that face, however the anisotropy of the shape can delimit this effect. Taken together, the simulation results on regular shapes provided crucial insights into the constraints operating on cortical microtubule array formation, as we could conceptualize the significant contribution of cell shape factors without assuming any genetic control.

Taking cell shape into account when considering microtubule dynamics enabled us to predict divisions in mostly non-growing embryonic cells (Yoshida et al. (2014); in silico, we made the simplifying assumption of no growth between $1 \rightarrow$ 16-cell stage) of the plant Arabidopsis (Chapter 5). In the absence of auxin signalling (i.e. in the $b d l$ mutant), the experimentally observed division plane pattern can be predicted via simulation of microtubule dynamics considering only edge-catastrophe. To explain the observed vertical division during the $1 \rightarrow 2$-cell stage transition, our simulation result indicated that a vertical division could be achieved by auxin mediated enhanced stabilization of microtubules at the developmentally new cell face. This result provides a strong hypothesis for molecular functions that might lead to the wild type division pattern of this and other cell stage transitions.

During preprophase band (PPB) formation, microtubules stochastically "search" (through increased dynamic instability without significant change in the microtubule length) for the domain of PPB in the cell cortex and get "captured" (through cross-linking activity at the PPB forming domain) upon 
its encounter. This is known as the "search and capture" mechanism of the formation of the preprophase band (PPB) (Vos et al., 2004). To translate these experimental observations in simulation, we took into consideration that invariance of the microtubule length indicates no change in the input control parameter $(G)$, which depends on the average microtubule length. Considering triggering of the offset, a compelling mechanism is increased nucleation in the cortex around the close vicinity of the nucleus. Therefore, using $G$ and nucleation rate as ingredients, we embedded the principle of the "search and capture" mechanism into our simulation framework (Chapter 5) by implementation of a stabilizing influence of the nuclear position on the location of the PPB, which is known to guide the insertion of division plane. This implementation allowed us to explain the first asymmetric division.

Finally, we inferred the necessity of one more rule that incorporates the effect of anisotropic growth/stress pattern in microtubule dynamics, to explain the divisions in the anisotropically growing cells, i.e. the elongating zygote and root epidermal cells of Arabidopsis (Chapter 6).

The Hofmeister (1863) rule assumed the new cell wall to be perpendicular to the principal growth direction. This rule is not applicable to the divisions in non-growing cells. The division rule proposed by Sachs (1878) states that new cell wall (face) is perpendicular to existing cell walls (faces). This rule creates ambiguity for cells with a large number of cell faces, as often the newly inserted face does not necessary intersect all the exiting faces. In such cases, the division predictions will be incomplete or can be considered as a partial prediction. The division rule suggested by Errera (1888) is based on the idea that the new cell wall will be a surface with minimum surface energy, and in computer simulations by Dupuy et al. (2010), the division rule was often approximated as the wall with minimum circumference, which often in the literature is called as shortest wall, passing through the centre of the cell. These two rules are:

(i) Ambiguous, particularly for irregular shapes, i.e plant cell shapes, as there are multiple possibilities of division plane with same minimum surface energy or circumference.

(ii) Not explicit about the orientation of the division plane, rather linked to the surface area or circumference of the division plane.

Therefore, for these two rules, we can approximate the corresponding orientation of the division plane and compare that with the experimentally observed division plane orientation. Following the above explained criteria, we examined the outcome of the different division rules against experimentally observed divisions plane orientation in non-growing embryonic cells and anisotropically growing root epidermis cells of Arabidopsis. 


\section{Geometric rule vs cell geometry based CMT simulation}

In many of our simulations, we did not consider any genetic/mechanical control factor that might influence the dynamics of microtubules, hence division plane orientation. Therefore corresponding simulation prediction of division plane orientation through microtubule array orientation can be appropriately compared with the division pattern observed under loss of function or perturbation in a key developmental controller (genetic or stress/growth) of oriented division. In this thesis, we performed only a genetic perturbation (leaving out mechanical perturbation such as modulation of anisotropic stress/growth for future research) towards oriented cell division, and in two different cell types:

1. The zygote and during the first five division steps of the $b d l$ apical 1-cell stage embryo, which has compromised auxin signalling

2. The root epidermal cells under PLT2 induction.

The observed division plane orientation under these genetic perturbations are likely to be a result of the oriented microtubule array, which is formed via basic regulatory effect of shape anisotropy and edge-catastrophe. Accordingly, in the Table 7.1, we compared the observed division plane orientation with the corresponding prediction of the different geometric rules of division and microtubule array orientation, where microtubule dynamics was simulated with cell geometry based rules, i.e. shape anisotropy and edgecatastrophe. The division rule proposed by Hofmeister (1863) predicts a

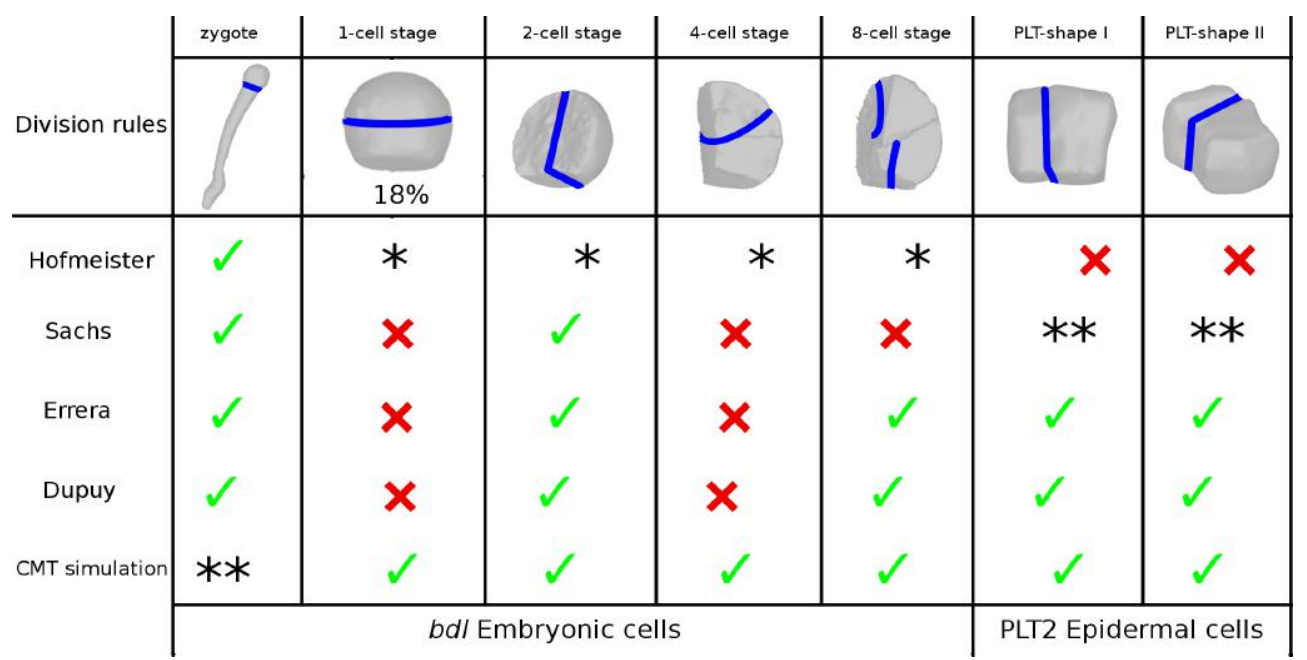

Table 7.1. Comparison of the different rules for cell division when a key genetic control in division is compromised. PLT-shape I: Epidermal cell that divides anticlinal-vertically under PLT2 induction and PLT-shape II: Epidermal cell that divides periclinally under PLT2 induction. Experimentally observed division plane orientations are shown by the blue (solid) lines. The symbols used in the table stands for: $\boldsymbol{V} \rightarrow$ correct prediction (green), $\boldsymbol{x} \rightarrow$ incorrect prediction (red), $\star \rightarrow$ not applicable and $\star \star \rightarrow$ incomplete/ partially prediction.

correct division only for the division of the zygote. Sachs's rule of division 
(Sachs, 1878) makes a correct division prediction only for the zygote and the 2-cell stage of the $b d l$ embryonic development. An approximation of the corresponding orientation of the division plane that results from the proposed rule of Errera (1888) or the rule used in the computer simulation of Dupuy et al. (2010), reveals that both these rules are able to give a match with the correct division for almost all the different cell types considered, except the 1-cell stage and 4-cell stage divisions of the $b d l$ embryonic development. Taken together, none of the proposed geometrical rules are able to predict the division plane orientation for the entire set of divisions, which occur during the first five division cycles of the $b d l$ embryos and PLT2 induced epidermal cells. The only division that is predicted correctly by all the different geometrical rules is division of the zygote. Interestingly, the simulated orientation of microtubule array is able to predict the division plane orientations for the entire set of cells, except the zygote and the 1-cell stage embryo, where the predictions are partial. To be specific,

- For the $b d l$ 1-cell stage embryo, the simulated microtubule array predicted only the experimentally observed partial horizontal division, leaving out the major fraction $(\approx 82 \%)$ of vertical divisions, which is also observed in experiments.

- For the zygote, only a fraction of simulated microtubule array orientation predicted the experimentally observed horizontal division. A major fraction $(\approx 56 \%)$ of simulated microtubule array orientations predicted vertical divisions, which is against experimental observation.

The experimentally observed vertical division $(\approx 82 \%)$ of the $b d l$ 1-cell stage embryo is correctly predicted (Sachs, 1878) or mapped (Errera, 1888; Dupuy et al., 2010) by all the geometrical rules, when applicable. The simulated array of microtubules is able to predict this vertical fraction of division only when, an additional rule of auxin mediated enhanced microtubule stabilization at the basal face is considered (see Table 7.2 , with auxin

\begin{tabular}{|c|c|c|c|c|c|c|}
\hline Division rules & Hofmeister & Sachs & Errera & Dupuy & \multicolumn{2}{|c|}{ CMT simulation } \\
\hline & * & $\checkmark$ & $\checkmark$ & $\checkmark$ & $x$ & $\checkmark$ \\
\hline $82 \%$ & & & & & & \\
\hline
\end{tabular}

Table 7.2. Comparison of the different rules for cell divisions (orientation) with the experimentally observed vertical division $(\approx 82 \%)$ of the $b d l$ 1-cell stage embryo. Experimentally observed division plane orientation is shown by the blue (solid) line. The symbols used in the table stands for: $\checkmark \rightarrow$ correct prediction (green), $\boldsymbol{x} \rightarrow$ incorrect prediction (red) and $\star \rightarrow$ not applicable.

rule). This suggests, in spite of genetic perturbation through $b d l$ mutation, a strong genetic control is retained, which influences the microtubule dynamics towards formation of the vertical array. For the zygote, the same auxin 
rule of enhanced microtubule stabilization can not be used as this cell does not contain multiple faces with different developmental age. This leaves us to search for another rule for microtubule dynamics that suppresses the simulated prediction of vertical divisions $(\approx 56 \%)$ of the zygote.

\section{Growth/Stress anisotropy based rule to overwrite geometric rules} To disregard the microtubule simulated vertical division $(\approx 56 \%)$ in the zygote, where implementation of an auxin rule is inapplicable, we could think about mechanical control (anisotropic stress/strain) on microtubule dynamics. Considering mechanical response, anisotropic growth/stress re-

\begin{tabular}{|c|c|c|c|c|c|c|}
\hline Division rules & Hofmeister & Sachs & Errera & Dupuy & \multicolumn{2}{|c|}{ CMT simulation } \\
\hline$\underbrace{}_{\substack{1-\text { cell stage } \\
\text { (wild type Embryonic cell) }}}$ & $*$ & $\sqrt{ }$ & $\checkmark$ & $\sqrt{ }$ & $x$ & $\checkmark$ \\
\hline & $\sqrt{ }$ & $* *$ & $x$ & $x$ & $x$ & $x$ \\
\hline $\begin{array}{c}\text { WT-sahpe } \\
\text { (Epidermal cell) }\end{array}$ & & & & & without auxin rule & with auxin rule \\
\hline
\end{tabular}

Table 7.3. Comparison of the different rules for cell divisions (orientation) in wild type condition. WT-shape: Wild type epidermal cell. Experimentally observed division plane orientations are shown by the blue (solid) lines. The symbols used in the table stands for: $\boldsymbol{V} \rightarrow$ correct prediction (green), $\boldsymbol{x} \rightarrow$ incorrect prediction (red), $\star \rightarrow$ not applicable and $\star \star \rightarrow$ incomplete/ partial prediction.

sponses of microtubules are relevant, specially because the zygote undergoes rapid anisotropic growth after fertilization of the egg cell. For example, the wild type root epidermal cell also grows anisotropically and subsequently all the geometrical rules of division fail to give a correct prediction of division, except the rule of Hofmeister. Here, the simulated microtubule array with auxin rule of microtubule stabilization at developmentally new cell faces, fails to give a correct division plane prediction (see Table 7.3 . with auxin rule). The simulation prediction, when possible influence of cell growth/stress in the microtubule dynamics was not considered, also turned out to be incorrect. The rule of Hofmeister is based on anisotropic growth (principle growth direction), and Table 7.3 indicates that such a specific rule for anisotropic growth is needed to overwrite many of the default geometric rules of division, possibly via influencing the default microtubule dynamics. This is in line with experimental evidence that anisotropic growth might also have a role in the reorientation of the microtubule array, thus influencing on the dynamics of microtubules (Uyttewaal et al., 2012; Landrein and Hamant, 2013; Louveaux et al., 2016). The wild type 1-cell stage embryo does not grow significantly and all the geometrical rules of division appear to give the experimentally observed vertical division, except the rule of Hofmeister. In this case, the simulated microtubule array with implementa- 
tion of auxin mediated microtubule stabilization at the basal face (see Table 7.3, with auxin rule), appears to be sufficient to predict the vertical division.

However, from the molecular perspective, the only partially correct prediction of division plane orientation for the zygote (or any elongated cell, i.e suspensor) and incorrect prediction of division for wild type epidermal cells make sense. These cells either grow significantly resulting in elongated in shape or are under strong anisotropic stress. These observations indicate that for anisotropically growing cells, the influence of anisotropic growth/stress in the dynamics of microtubules needs to be considered explicitly. Therefore, the next step in modelling will be to simulate microtubule dynamics on growing cell surfaces, which is possible by implementing the Finite Element Method (Reddy, 2001) on triangulated approximations of the cell shape. However, simulations that incorporate growth effects in microtubule dynamics require further experimental knowledge on growth/stress responses of microtubules such as via stress mediated induction of katanin, which severs microtubules at crossover sites (Uyttewaal et al., 2012; Landrein and Hamant, 2013; Louveaux et al., 2016). For non-growing or slow growing cells, the auxin rule of enhanced microtubule stabilization at the developmentally new cell face(s) might be sufficient to give correct division plane orientation for wild type cells. However, as plant cells acquire multiple faces inserted at different developmental time points, the distribution of microtubule stabilizing factors at the different cell faces might vary significantly. Therefore, experimental determination of the density of such microtubule stabilizing factors at the cortex of different cell faces will be interesting. Ideally, future improvements in imaging technology may allow us to directly measure microtubule dynamics at different cell surfaces in vivo.

Our modelling prediction of the formation of the preprophase band (PPB) is based on compelling experimental observations, but certain hypotheses, such as favoured microtubule nucleation at the PPB forming domain of the cortex need more detailed experimental confirmation. Based on our simulation result in the context of elongating/growing cells, we speculated about the influence of anisotropic stress distribution in the dynamics of microtubules. A good way to measure such influences will be by experiments that modulate anisotropic cell growth inhibition (Scheible et al., 2001; Harris et al., 2012; Worden et al., 2015), and quantify the frequency of division plane reorientation. In the growing epidermal cells, the transcriptional regulation of division plane reorientation stimulates a further experimental search for the genetic regulation underneath microtubule dynamics, i.e how does auxin signalling via TIR1 genes (Ruegger et al., 1998) or CLASP (Ambrose et al. 2007) lead to changes in activity of microtubule associated proteins. 
In summary, in the absence of key genetic control of oriented cell division, the best accuracy in predicting the division plane orientation in Arabidopsis cells is obtained through the simulated array of microtubules, where the microtubules are individual molecular aggregates. This brings a significant insight towards the molecular understanding of oriented cell division in plant cells, and therefore on mechanisms that guide efficient plant morphogenesis. The differently regulated embryonic divisions observed in monocots such as maize (Clark and Sheridan, 1988; Costa et al., 2003; Magnard et al., 2004; Wright et al., 2009; Phillips and Evans, 2011) can possibly be addressed using our understanding of divisions in the embryos of Arabidopsis, combined with yet to be fully substantiated mechanisms that control the division plane orientation when cells undergo anisotropic stress. In this way, we may be able to generalize molecular principles of oriented cell division to many other plants. 


\section{Supplementary results}

\section{S.1 Case study for 1-cell stage $b d l$ embryo of Arabidopsis}

To get a hint towards understanding the experimentally observed vertical division during $1 \rightarrow 2$-cell stage transitions, here we investigate a few possibilities.

\section{S.1.1 Default shape simulation}

In this section, we describe our simulation result of microtubule dynamics on the default shape. Simulated orientation of microtubule array showed apparently random distribution in $b d l$ mutant (see Fig. S.1). When we
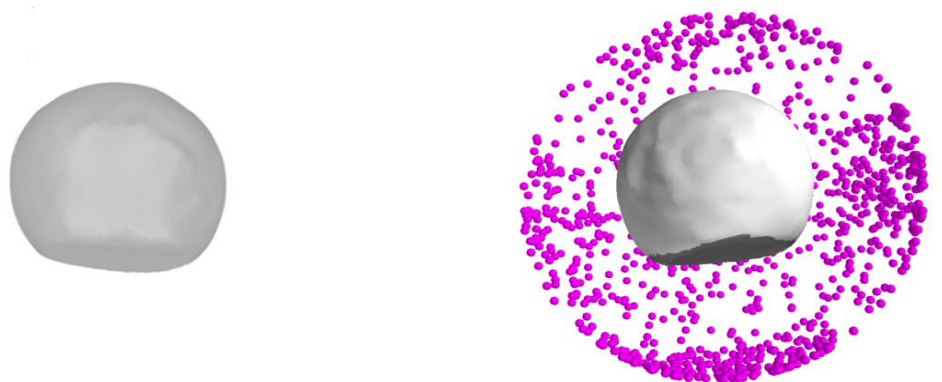

Fig. S.1. Simulated orientation of microtubule array on default 1-cell stage embryonic shape of $A r a-$ bidopsis. Simulation was performed for $\approx 1000$ different configurations. Each dot point represents the tip of an orientation vector associated with a simulation configuration, and perpendicular to an associated microtubule array. We found almost random distribution of microtubule array orientation. Simulation was performed on cell shape that was imaged in the $b d l$ mutation. Cell templates used in these simulations was provided by Thijs de Zeeuw (WUR).

compared this simulation result with the corresponding wild type shape simulation result, we found that the $b d l 1$-cell shape simulation showed higher degree of randomness in the microtubule array orientation distribution. By comparing the degree of shape anisotropy present between the wild type 1-cell stage and the $b d l$ 1-cell stage embryos, we found that the wild type 1-cell stage shape is a bit elongated towards the vertical direction than the corresponding $b d l$ shape. It is interesting to see that, even such little difference in shape anisotropy can have an impact on the orientation of the microtubule arrays.

\section{S.1.2 Combined effect of edge-catastrophe and enhanced microtubule stabilization at the basal face}

In this section, we describe our simulation result of microtubule dynamics with edge-catastrophe and enhanced stabilization of microtubules at the 
basal face. Simulated microtubule arrays showed a majority of horizontal

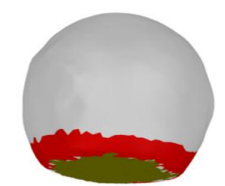

enhanced face stability

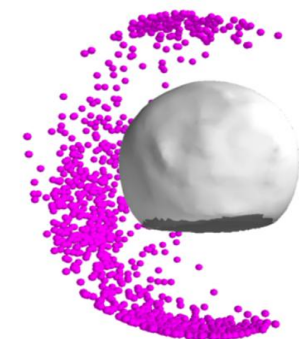

Fig. S.2. Simulated orientation of microtubule array on embryonic cell shape of Arabidopsis during $1 \rightarrow$ 2-cell stage transition, with edge catastrophe at the edge and enhanced stabilization of microtubules at the basal face. Simulation was performed for $\approx 1000$ different configurations. Each dot point represents the tip of an orientation vector associated with a simulation configuration, and perpendicular to an associated microtubule array. We found two clusters of microtubule array orientation: horizontal $\approx$ $62 \%$ (607/981), vertical $\approx 38 \%$ (374/981). Stabilization of microtubules was given via reduced value of dynamics catastrophe rate $\left(r_{c}\right)$. In this particular simulation we implemented a maximum of 100 times reduction in $r_{c}$ at the basal face, which was still unable to give an anticipated full $100 \%$ vertical orientation. Cell edge is coloured in red to indicate that in simulation microtubules were subjected to edge-catastrophe. Simulation was performed on cell shape that was imaged in the $b d l$ mutation. Cell templates used in these simulations was provided by Thijs de Zeeuw (WUR).

orientation with a small fraction of vertical orientation (see Fig. S.2). This simulation result indicated that enhanced stabilization of microtubules at the basal face has the potential to generate vertical orientation of microtubule array, however presence of edge-catastrophe might suppress the actual effect.

\section{S.1.3 Effect of enhanced stabilization of microtubules at the basal face}

In this section, we describe our simulation result of microtubule dynamics with enhanced microtubule stabilization at the basal face without edgecatastrophe in the dynamics of microtubules. As shown in Fig. S.3, in the

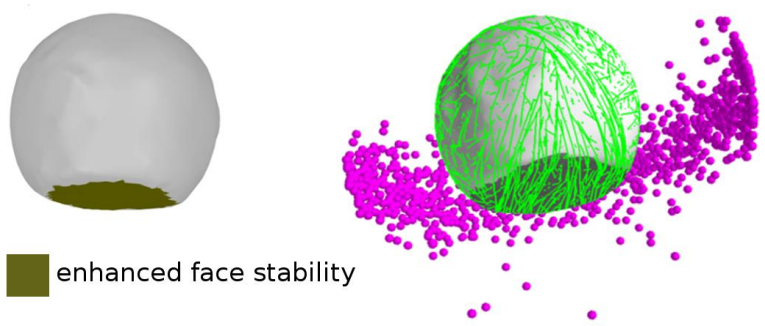

Fig. S.3. Simulated orientation of microtubule array on embryonic cell shape of Arabidopsis during $1 \rightarrow 2$-cell stage transitions, with enhanced stabilization of microtubules at the basal face. Simulation was performed for $\approx 1000$ different configurations. Each dot point represents the tip of an orientation vector associated with a simulation configuration, and perpendicular to an associated microtubule array. We found only one cluster of microtubule array orientation, which was vertical $\approx 98 \%(971 / 991)$. Microtubule stabilization was given via a reduced value of dynamics catastrophe rate $\left(r_{c}\right)$. For consistency with previous simulations, we implemented a maximum 100 times reduction in $r_{c}$ at the basal face. However, We observed a 5-10 times reduction in $r_{c}$ at the basal face was sufficient to generate a full vertical microtubule array orientation. Simulation was performed on cell shape that was imaged in the $b d l$ mutation. Cell templates used in these simulations was provided by Thijs de Zeeuw (WUR). 
absence of edge-catastrophe, enhanced microtubule stabilization at the basal face was sufficient to generate a full vertical orientation of the microtubule array.

\section{S.1.4 Effect of microtubule stabilizing polar cap}

In the context of microtubule stabilization, biological reference to a microtubule stabilizing apical domain (i.e. a microtubule stabilizing polar cap) needs to be determined. In this section, we use our modelling framework

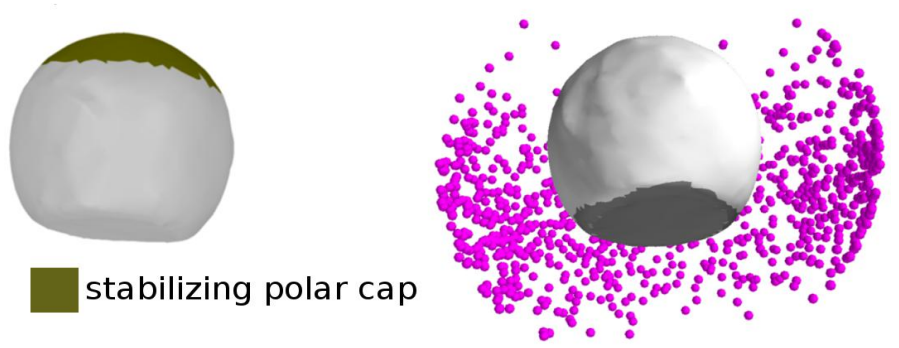

Fig. S.4. Simulated orientation of microtubule array on embryonic cell shape of Arabidopsis during $1 \rightarrow 2$-cell stage transitions, with microtubule stabilizing apical domain (i.e a microtubule stabilizing polar cap). Simulation was performed for $\approx 1000$ different configurations. Each dot point represents the tip of an orientation vector associated with a simulation configuration, and perpendicular to an associated microtubule array. We found apparently random distribution of microtubule array orientation. Microtubule stabilization at the apical polar cap was given via a reduced value of dynamics catastrophe rate $\left(r_{c}\right)$. For consistency with previous simulations, we implemented a maximum of 100 times reduction in $r_{c}$ at the basal face, which was still unable to give an anticipated vertical microtubule array orientation. Simulation was performed on cell shape that was imaged in the $b d l$ mutation. Cell templates used in these simulations was provided by Thijs de Zeeuw (WUR).

to explore the effect of such a microtubule stabilizing polar cap on the microtubule array orientation. Accordingly, we simulated microtubule dynamics by defining an apical domain that provides enhanced microtubule stabilization. For the same range of the degree of enhanced microtubule stabilization (as used for simulation with enhanced microtubule stabilization at the basal face), the microtubule stabilizing apical cap failed to generate a vertical distribution of microtubule array orientation (see Fig. S.4). For comparison with the simulation outcome with microtubule stabilization at the basal face (Sec. S.1.3), we restricted the area of the microtubule stabilizing apical domain to be equivalent to the area of the basal face.

\section{S.2 Simulating the PPB formation mechanism}

To understand the formation of the preprophase band (PPB), here we describe our simulation implementation of the "search and capture" mechanism (Vos et al., 2004). Starting from an ordered sparse array of microtubules, we triggered a small offset of increased nucleation rate at the surface domain (cortex) that resides at the close vicinity of the nucleus. 


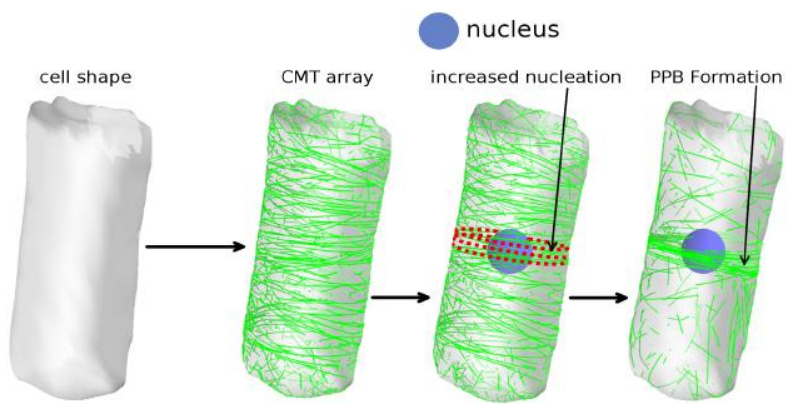

Fig. S.5. Simulation of the PPB formation mechanism. First, microtubule dynamics was simulated on the cell shape with basic rules of microtubule dynamics and integration of regulatory effects. In this case the regulatory effect was inclusion of edge-catastrophe in the dynamics of microtubules, which leads to the formation of an order array of microtubule at steady state (CMT array). Assuming the nucleus to be located at the center of the cell, immediately upon formation of the ordered array of microtubules, we triggered a small offset of increased nucleation rate $(\approx 20 \%)$ at the shape surface (cortex) that resides at the close vicinity of the nucleus (increased nucleation; red dotted domain). Within an hour (biological time) of the triggering the offset, the sparse array of microtubule collapsed into a dense ring of microtubule, i.e the PPB (PPB formation). Cell templates used in these simulations was provided by Viola Willemsen (WUR).

Upon triggering of the offset, within one hour (biological time), we observed the formation of a dense ring of microtubules from the initial sparse array (see Fig. S.5). These dense ring can be viewed as a resemble of the preprophase band (PPB).

\section{S.3 Sensitivity of microtubule array orientation to embryonic cell shape anisotropy}

For both wild type and $b d l$ shapes, simulation on 2-cell stage cell without edge-catastrophe in microtubule dynamics, predicted two possibilities (horizontal/vertical) of microtubule array orientation. On the same shapes, with inclusion of edge-catastrophe in microtubule dynamics, the two possibilities were reduced to a single possibility (one cluster of microtubule array orientation). The average orientation of the microtubule array correctly predicted the experimentally observed vertical division. However, in the $b d l$ shape, a noticeable spreading was observed in the cluster of microtubule array orientation of the microtubule array orientation vector tips. This intrigued us to look at the geometrical details of these cell shapes. Accordingly, we made a comparison and found that in wild type shape the longitudinal dimension was greater than the lateral dimension, but in the $b d l$ shape the longitudinal and lateral dimensions were almost equal (see Fig. S.6). In wild type shape, relatively shorter lateral dimension leads to more frequent encountering of the microtubules with the edges. Thus more edge-catastrophe along the lateral direction, resulting in increasing possibility of array formation along the longitudinal (vertical) direction, which is a path of less frequent edge-catastrophe. In $b d l$ shape, comparable 


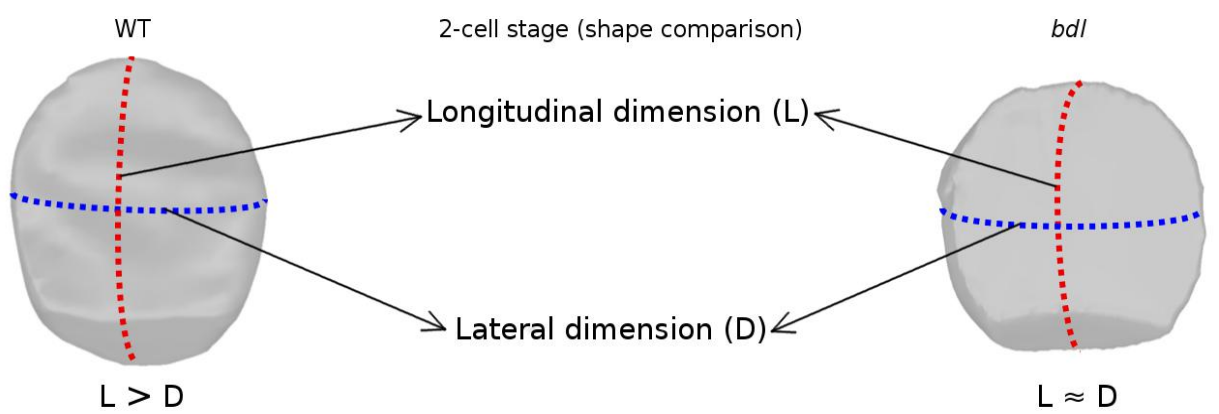

Fig. S.6. Effect of the $b d l$ mutation on the cell shapes of Arabidopsis embryo. The cell shape at 2-cell stage $b d l$ embryo was compared with the corresponding wild type shape. In wild type case, longitudinal dimension $(\mathrm{L})$ was greater that the lateral dimension $(L>D)$. In the $b d l$ induced case, the dimensions were almost equal $(L \approx D)$. When microtubule dynamics was simulated with edge-catastrophe enabled, the difference in shape did not alter the average orientation of the microtubule array, but a noticeable difference was observed in the variation around the mean orientation (see Fig. 5.3 vs Fig. 5.5, 2-cell stage). Cell templates used in these simulations was provided by Viola Willemsen (WUR).

lateral and longitudinal dimension did not favour any of the two directions, in terms of the frequency of encountering an edge. However, the overall extent and curvature values of the edges along the lateral dimension still managed to favour possibilities of microtubule array formation along the longitudinal (vertical) path. This explains the origin of the spread in the cluster of microtubule array orientations in $b d l$ shape. 


\section{Appendix}

\section{A.1 Geometric measures on a triangulated shape}

If $\Gamma \in \mathbb{R}$ is a region in space occupied by a three dimensional object with uniform material density $\rho(x, y, z)=1$. Then, we can define the three dimensional moment of order $(k+l+m)$ as,

$$
\begin{aligned}
Q^{(k l m)}(\Gamma) & =\int_{\Gamma} \rho(x, y, z) x^{k} y^{l} z^{m} d \Gamma \\
& =\int_{\Gamma} x^{k} y^{l} z^{m} d \Gamma[\operatorname{as} \rho(x, y, z)=1] \\
& =\int_{\Gamma} \vec{\nabla} \cdot \vec{V}^{(k l m)} d \Gamma \\
& =\oint_{S} \vec{V}^{(k l m)} \cdot \hat{n} d S
\end{aligned}
$$

where,

$$
\vec{\nabla} \cdot \vec{V}^{(k l m)}=\frac{\partial V_{1}^{(k l m)}(x, y, z)}{\partial x}+\frac{\partial V_{2}^{(k l m)}(x, y, z)}{\partial y}+\frac{\partial V_{3}^{(k l m)}(x, y, z)}{\partial z}=x^{k} y^{l} z^{m}
$$

An acceptable solution for $V_{x}, V_{y}$ and $V_{z}$ is,

$$
\begin{aligned}
V_{1}^{(k l m)}(x, y, z) & =\frac{x^{k+1} y^{l} z^{m}}{3(k+1)}, \\
V_{2}^{(k l m)}(x, y, z) & =\frac{x^{k} y^{l+1} z^{m}}{3(l+1)}, \\
V_{3}^{(k l m)}(x, y, z) & =\frac{x^{k} y^{l} z^{m+1}}{3(m+1)}
\end{aligned}
$$

For a three dimensional triangulated surface mesh, the total moment of the region enclosed by the triangle mesh can be expressed as,

$$
\begin{aligned}
Q^{(k l m)}(\Gamma) & =Q^{(k l m)}(S) \\
& =\oint_{S} \vec{V}^{(k l m)} \cdot \hat{n} d S \\
& =\sum_{i=1}^{N} \oint_{A_{i}} \vec{V}^{(k l m)} \cdot \hat{n}_{i} d A_{i} \\
& =\sum_{i=1}^{N} Q^{(k l m)}\left(T_{i}\right)
\end{aligned}
$$


where, $N$ is total number of triangles in the mesh and $A_{i}$ is the area of the $i^{i t}$ triangle $\left(T_{i}\right)$. Further simplification gives,

$$
Q^{(k l m)}\left(T_{i}\right)=\oint_{A_{i}} \vec{V}^{(k l m)} \cdot \hat{n}_{i} d A_{i}=\sum_{j=1}^{3} n_{i j} \oint_{A_{i}} V_{j}^{(k l m)} d A_{i}=\sum_{j=1}^{3} n_{i j} T_{i j}
$$

where,

$$
n_{i j}=\hat{j} \cdot \hat{n}_{i}
$$

and

$$
T_{i j}=\oint_{A_{i}} V_{j}^{(k l m)} d A_{i}
$$

Now, for the $i^{\text {th }}$ triangle, we can use the barycentric coordinates $\left(x_{b}, y_{b}\right)$, where $0 \leq x_{b}, y_{b} \leq 1$ and $0 \leq x_{b}+y_{b} \leq 1$, to position a point inside the triangle by,

$$
\begin{aligned}
& x_{i}\left(x_{b}, y_{b}\right)=\left(x_{i}^{(2)}-x_{i}^{(1)}\right) x_{b}+\left(x_{i}^{(3)}-x_{i}^{(1)}\right) y_{b}-x_{i}^{(1)} \\
& y_{i}\left(x_{b}, y_{b}\right)=\left(y_{i}^{(2)}-y_{i}^{(1)}\right) x_{b}+\left(y_{i}^{(3)}-y_{i}^{(1)}\right) y_{b}-y_{i}^{(1)} \\
& z_{i}\left(x_{b}, y_{b}\right)=\left(z_{i}^{(2)}-z_{i}^{(1)}\right) x_{b}+\left(z_{i}^{(3)}-z_{i}^{(1)}\right) y_{b}-z_{i}^{(1)}
\end{aligned}
$$

Having this, we can express Eqn. A.5 as,

$$
T_{i j}=\oint_{A_{i}} V_{j}^{(k l m)} d A_{i}=2 A_{i} \int_{0}^{1} \int_{0}^{\left(1-x_{b}\right)} V_{j}^{(k l m)}\left(T_{i}\right) d x_{b} d y_{b}
$$

where the integrand $V_{j}^{(k l m)}\left(T_{i}\right)$ is defined as,

$$
V_{j}^{(k l m)}\left(T_{i}\right)=\frac{1}{3}\left\{\begin{array}{l}
\frac{1}{(k+1)} x_{i}^{k+1}\left(x_{b}, y_{b}\right) y_{i}^{l}\left(x_{b}, y_{b}\right) z_{i}^{m}\left(x_{b}, y_{b}\right), j=1 \\
\frac{1}{(l+1)} x_{i}^{k}\left(x_{b}, y_{b}\right) y_{i}^{l+1}\left(x_{b}, y_{b}\right) z_{i}^{m}\left(x_{b}, y_{b}\right), j=2 \\
\frac{1}{(m+1)} x_{i}^{k}\left(x, y_{b}\right) y_{i}^{l}\left(x_{b}, y_{b}\right) z_{i}^{m+1}\left(x_{b}, y_{b}\right), j=3
\end{array}\right.
$$

Following Eqn. A.4.Eqn. A.6, we get

$$
Q^{(k l m)}\left(T_{i}\right)=2 A_{i} \int_{0}^{1} \int_{0}^{\left(1-x_{b}\right)} V_{j}^{(k l m)}\left(T_{i}\right) d x_{b} d y_{b}
$$

Using Eqn. A.3 and Eqn. A.7, we get the moment of the triangulated polyhedral,

$$
Q^{(k l m)}(S)=\sum_{i=1}^{N} Q^{(k l m)}\left(T_{i}\right)=\sum_{i=1}^{N} 2 A_{i} \int_{0}^{1} \int_{0}^{\left(1-x_{b}\right)} V_{j}^{(k l m)}\left(T_{i}\right) d x_{b} d y_{b}
$$


From here on we assume,

$$
Q^{(k l m)}(\Gamma)=Q^{(k l m)}(S)=Q^{(k l m)}=\sum_{i=1}^{N} Q^{(k l m)}\left(T_{i}\right)
$$

\section{Volume}

Total volume occupied by the object,

$$
M_{\Gamma}=\int_{\Gamma} d \Gamma=\int_{\Gamma} x^{0} y^{0} z^{0} d \Gamma=\sum_{i=1}^{N} Q^{(000)}\left(T_{i}\right)
$$

\section{Centroid}

Centroid $\left(x_{C}, y_{C}, z_{C}\right)$ of the object,

$$
\begin{aligned}
& x_{C}=\frac{\int_{\Gamma} x d \Gamma}{\int_{\Gamma} d \Gamma}=\frac{\int_{\Gamma} x^{1} y^{0} z^{0} d \Gamma}{\int_{\Gamma} x^{0} y^{0} z^{0} d \Gamma}=\frac{Q^{(100)}(\Gamma)}{Q^{(000)}(\Gamma)}=\frac{\sum_{i=1}^{N} Q^{(100)}\left(T_{i}\right)}{\sum_{i=1}^{N} Q^{(000)}\left(T_{i}\right)}, \\
& y_{C}=\frac{\int_{\Gamma} y d \Gamma}{\int_{\Gamma} d \Gamma}=\frac{\int_{\Gamma} x^{0} y^{1} z^{0} d \Gamma}{\int_{\Gamma} x^{0} y^{0} z^{0} d \Gamma}=\frac{Q^{(010)}(\Gamma)}{Q^{(000)}(\Gamma)}=\frac{\sum_{i=1}^{N} Q^{(010)}\left(T_{i}\right)}{\sum_{i=1}^{N} Q^{(000)}\left(T_{i}\right)}, \\
& z_{C}=\frac{\int_{\Gamma} z d \Gamma}{\int_{\Gamma} d \Gamma}=\frac{\int_{\Gamma} x^{0} y^{0} z^{1} d \Gamma}{\int_{\Gamma} x^{0} y^{0} z^{0} d \Gamma}=\frac{Q^{(001)}(\Gamma)}{Q^{(000)}(\Gamma)}=\frac{\sum_{i=1}^{N} Q^{(001)}\left(T_{i}\right)}{\sum_{i=1}^{N} Q^{(000)}\left(T_{i}\right)}
\end{aligned}
$$

\section{Symmetry directors}

The inertia tensor,

$$
I=\left[\begin{array}{lll}
I_{x x} & I_{x y} & I_{x z} \\
I_{y x} & I_{y y} & I_{y z} \\
I_{z x} & I_{z y} & I_{z z}
\end{array}\right]
$$

with tensor components,

$$
\begin{aligned}
I_{x x} & =\int_{\Gamma}\left(y^{2}+z^{2}\right) d \Gamma \\
& =Q^{(020)}(\Gamma)+Q^{(002)}(\Gamma) \\
& =\sum_{i=1}^{N} Q^{(020)}\left(T_{i}\right)+\sum_{i=1}^{N} Q^{(002)}\left(T_{i}\right) \\
I_{y y} & =\int_{\Gamma}\left(x^{2}+z^{2}\right) d \Gamma \\
& =Q^{(200)}(\Gamma)+Q^{(002)}(\Gamma) \\
& =\sum_{i=1}^{N} Q^{(200)}\left(T_{i}\right)+\sum_{i=1}^{N} Q^{(002)}\left(T_{i}\right)
\end{aligned}
$$




$$
\begin{aligned}
I_{z z} & =\int_{\Gamma}\left(x^{2}+y^{2}\right) d \Gamma \\
& =Q^{(200)}(\Gamma)+Q^{(020)}(\Gamma) \\
& =\sum_{i=1}^{N} Q^{(200)}\left(T_{i}\right)+\sum_{i=1}^{N} Q^{(020)}\left(T_{i}\right)
\end{aligned}
$$

$$
\begin{aligned}
I_{x y}=I_{y x} & =\int_{\Gamma} x y d \Gamma \\
& =Q^{(110)}(\Gamma) \\
& =\sum_{i=1}^{N} Q^{(110)}\left(T_{i}\right)
\end{aligned}
$$

$$
\begin{aligned}
I_{y z}=I_{z y} & =\int_{\Gamma} y z d \Gamma \\
& =Q^{(011)}(\Gamma) \\
& =\sum_{i=1}^{N} Q^{(011)}\left(T_{i}\right)
\end{aligned}
$$

$$
\begin{aligned}
I_{z x}=I_{x z} & =\int_{\Gamma} z x d \Gamma \\
& =Q^{(101)}(\Gamma) \\
& =\sum_{i=1}^{N} Q^{(101)}\left(T_{i}\right)
\end{aligned}
$$

Each of the three vectors corresponding to the three eigenvalues of the inertia tensor $(I)$, will be an axis of symmetry, i.e symmetry director.

\section{Measure of edge-angle (curvature)}

In a triangulated surface, two faces adjacent to a domain of sharp curvature (i.e an edge), will be an approximation of many triangles. As a result, the edge will be a combination of many triangles. For each of the two adjacent faces at an edge, we assign an average face normal. The average face normal is a vector, having an average orientation of the normals of all the triangles associated with a face. For a flat face, the average face normal will reflect the orientation of the individual triangle face normals. For a curved face, individual orientation of the triangle normals associated to the edge will vary a lot with respect to each other. This will result in an average orientation of the face normal that does not necessarily reflect the orientation of the individual triangle face normals. Here, we calculate the average edge-angle $\left(\theta_{E d g}^{a v g}\right.$ ) for the following cases: 
1. Consider an edge, which connects two flat faces $K_{1}$ and $K_{2}$. If the faces are with average face normal $\hat{n}_{K_{1}}$ and $\hat{n}_{K_{2}}$, we can calculate,

$$
\theta_{E d g}^{a v g}=\theta_{E d g}^{\left(K_{1}-K_{2}\right)}=\cos ^{-1}\left(\hat{n}_{K_{1}} \cdot \hat{n}_{K_{2}}\right)
$$

2. Consider an edge, which connects a flat face $K$ with a curved face $S$. For the curved face $S$, we can use the dot product of the flat face (average) normal $\hat{n}_{K}$ and the curved face local normals $\hat{n}_{l}^{(S)}(l=$ $1,2,3, \ldots N)$ to calculate the local edge-angles, where $N$ is the total number of local triangles associated with the curved face $S$, and fall in the domain of the edge. An average over these local edge-angles will give,

$$
\theta_{E d g}^{a v g}=\theta_{E d g}^{(K-S)}=\frac{\sum_{l=1}^{N} \cos ^{-1}\left(\hat{n}_{K} \cdot \hat{n}_{l}^{(S)}\right)}{N}
$$

\section{Normalization of local edge-angles}

For each triangle-pair $\left(T_{i}, T_{j}\right)$ with normal $\hat{n}_{i}$ and $\hat{n}_{j}$, the local edge-angle between the triangle pairs is,

$$
\theta_{e d g}^{\left(T_{i}, T_{j}\right)}=\cos ^{-1}\left(\hat{n}_{i} \cdot \hat{n}_{j}\right)
$$

At a give edge, using the value of the edge-angle $\theta_{E d g}^{a v g}$ and $\theta_{e d g}^{T_{i}, T_{j}}$, we define a weight factor,

$$
w_{e d g}^{(i, j)}=\frac{1}{\theta_{E d g}^{a v g}} \cos ^{-1}\left(\hat{n}_{i} \cdot \hat{n}_{j}\right)
$$

This is also a measure of normalized value of edge-angle between the triangle pair $\left(T_{i}, T_{j}\right)$, normalized with respect to the average edge-angle $\theta_{E d g}^{a v g}$.

\section{A.2 Simulation technicalities}

\section{Quaternion rotation of triangles}

To rotate $\triangle A B C$ from three dimensional $x-y-z$ space to two dimensional $x-y$ plane, we define an appropriate rotation operator, a quaternion $\mathbf{Q}_{(\triangle A B C)}$. We also define a unit outward normal to the plane of $\triangle A B C$,

$$
\hat{n}^{(\triangle A B C)}=\frac{\overrightarrow{A C} \times \overrightarrow{B A}}{|\overrightarrow{A C} \times \overrightarrow{B A}|}
$$

Using a unit vector $\hat{z}$ along $z$-axis, we calculate, 
1. The angle of rotation: $\theta_{\text {rot }}^{(\triangle A B C)}=\cos ^{-1}\left(\hat{n}^{(\triangle A B C)} \cdot \hat{z}\right)$

2. The axis of rotation: $\hat{u}^{(\triangle A B C)}=\hat{n}^{(\triangle A B C)} \times \hat{z}$

Using $\theta_{\text {rot }}^{(\triangle A B C)}, \hat{u}^{(\triangle A B C)}$ and an extension of Euler's formula, we construct the required rotation operator, i.e. the quaternion,

$$
\begin{aligned}
\mathbf{Q}_{(\triangle A B C)} & =e^{\frac{\theta_{\text {rot }}^{(\triangle A B C)}}{2}}\left(u_{x}^{(\triangle A B C)} \hat{i}+u_{y}^{(\triangle A B C)} \hat{j}+u_{z}^{(\triangle A B C)} \hat{k}\right) \\
& =\cos \left(\frac{\theta_{\text {rot }}^{(\triangle A B C)}}{2}\right)+\left(u_{x}^{(\triangle A B C)} \hat{i}+u_{y}^{(\triangle A B C)} \hat{j}+u_{z}^{(\triangle A B C)} \hat{k}\right) \sin \left(\frac{\theta_{\text {rot }}^{(\triangle A B C)}}{2}\right)
\end{aligned}
$$

In matrix form,

$$
\mathbf{Q}_{(\triangle A B C)}=\left[\begin{array}{c}
\cos \left(\frac{\theta_{\text {rot }}^{(\triangle A B C)}}{2}\right) \\
\sin \left(\frac{\theta_{\text {rot }}^{(\triangle A B C)}}{2}\right) u_{x}^{(\triangle A B C)} \\
\sin \left(\frac{\theta_{\text {rot }}^{(\Delta A B C)}}{2}\right) u_{y}^{(\triangle A B C)} \\
\sin \left(\frac{\theta_{\text {rot }}^{(\Delta A B C)}}{2}\right) u_{z}^{(\triangle A B C)}
\end{array}\right]
$$

Similarly, we construct the corresponding inverse quaternion,

$\mathrm{Q}_{(\triangle A B C)}^{-\mathbf{1}}=\cos \left(\frac{\theta_{\text {rot }}^{(\triangle A B C)}}{2}\right)-\left(u_{x}^{(\triangle A B C)} \hat{i}+u_{y}^{(\triangle A B C)} \hat{j}+u_{z}^{(\triangle A B C)} \hat{k}\right) \sin \left(\frac{\theta_{\text {rot }}^{(\triangle A B C)}}{2}\right)$

In matrix form,

$$
\mathbf{Q}_{(\triangle A B C)}^{-1}=\left[\begin{array}{c}
\cos \left(\frac{\theta_{\text {rot }}^{(\triangle A B C)}}{2}\right) \\
-\sin \left(\frac{\theta_{\text {rot }}^{(\Delta A B C)}}{2}\right) u_{x}^{(\triangle A B C)} \\
-\sin \left(\frac{\theta_{\text {rot }}^{(\triangle A B C)}}{2}\right) u_{y}^{(\triangle A B C)} \\
-\sin \left(\frac{\theta_{\text {rot }}^{(\triangle A B C)}}{2}\right) u_{z}^{(\triangle A B C)}
\end{array}\right]
$$

Using combination of $\mathbf{Q}_{(\triangle A B C)}$ and $\mathbf{Q}^{-\mathbf{1}}(\triangle A B C)$, we rotate $\triangle A B C$ from present orientation, i.e. $\left.\hat{n}^{(\triangle A B C)}\right)$, to a new orientation, $\hat{n}^{(\triangle A B C)} \| \hat{z}$. Rotation of $\triangle A B C$ can be performed by applying the quaternion rotation $\mathbf{Q}_{(\triangle A B C)}$ to each of the triangle vertices $A\left(X_{1}, Y_{1}, Z_{1}\right), B\left(X_{2}, Y_{2}, Z_{2}\right)$ and $C\left(X_{3}, Y_{3}, Z_{3}\right)$ separately. To rotate these vertices, first we express them in quaternion 
form,

$$
\begin{aligned}
\mathbf{Q}_{(A)} & =e^{\frac{\pi}{2}\left(A_{x} \hat{i}+A_{y} \hat{j}+A_{z} \hat{k}\right)} \\
& =0+\left(X_{1} \hat{i}+Y_{1} \hat{j}+Z_{1} \hat{k}\right) \\
\mathbf{Q}_{(B)} & =e^{\frac{\pi}{2}\left(B_{x} \hat{i}+B_{y} \hat{j}+B_{z} \hat{k}\right)} \\
& =0+\left(X_{2} \hat{i}+Y_{2} \hat{j}+Z_{2} \hat{k}\right) \\
\mathbf{Q}_{(C)} & =e^{\frac{\pi}{2}\left(C_{x} \hat{i}+C_{y} \hat{j}+C_{z} \hat{k}\right)} \\
& =0+\left(X_{3} \hat{i}+Y_{3} \hat{j}+Z_{3} \hat{k}\right)
\end{aligned}
$$

In matrix form,

$$
\mathbf{Q}_{(A)}=\left[\begin{array}{c}
0 \\
X_{1} \\
Y_{1} \\
Z_{1}
\end{array}\right], \mathbf{Q}_{(B)}=\left[\begin{array}{c}
0 \\
X_{2} \\
Y_{2} \\
Z_{2}
\end{array}\right] \text { and } \mathbf{Q}_{(C)}=\left[\begin{array}{c}
0 \\
X_{3} \\
Y_{3} \\
Z_{3}
\end{array}\right]
$$

After quaternion rotation, the surface of $\triangle A B C$ is now parallel to $x-y$ plane with vertices having a constant value of $z$-coordinate say,

$$
z=z_{(\triangle A B C)}^{o f f}
$$

Further, applying an appropriate $z$-axis translation, we place $\triangle A B C$ exactly on the two dimensional $x-y$ plane, i.e making $z=0$. After the rotation (if necessary, also the $z$-axis translation), the vertices acquire their new position,

$$
\begin{aligned}
& A\left(X_{1}, Y_{1}, Z_{1}\right) \rightarrow A\left(x_{1}, y_{1}, 0\right) \\
& B\left(X_{2}, Y_{2}, Z_{2}\right) \rightarrow B\left(x_{2}, y_{2}, 0\right) \\
& C\left(X_{3}, Y_{3}, Z_{3}\right) \rightarrow C\left(x_{3}, y_{3}, 0\right)
\end{aligned}
$$

where,

$$
\begin{aligned}
& {\left[\begin{array}{c}
0 \\
x_{1} \\
y_{1} \\
0
\end{array}\right]=\mathbf{Q}_{(\triangle A B C)} \mathbf{Q}_{(A)} \mathbf{Q}^{-\mathbf{1}}{ }_{(\triangle A B C)}} \\
& {\left[\begin{array}{c}
0 \\
x_{2} \\
y_{2} \\
0
\end{array}\right]=\mathbf{Q}_{(\triangle A B C)} \mathbf{Q}_{(B)} \mathbf{Q}_{(\triangle A B C)}^{-\mathbf{1}}{ }_{(\triangle A B C)}} \\
& {\left[\begin{array}{c}
0 \\
x_{3} \\
y_{3} \\
0
\end{array}\right]=\mathbf{Q}_{(\triangle A B C)} \mathbf{Q}_{(C)} \mathbf{Q}^{-\mathbf{1}}{ }_{(\triangle A B C}}
\end{aligned}
$$


Appendix

\section{Affine transformation mapping}

For $\triangle A D C$ with vertices $A\left(x_{1}^{\prime}, y_{1}^{\prime}\right), C\left(x_{2}^{\prime}, y_{2}^{\prime}\right)$ and $D\left(x_{3}^{\prime}, y_{3}^{\prime}\right)$, we define following two matrices,

$$
\mathbf{F}_{(\triangle A D C)}=\left[\begin{array}{cc}
x_{2}^{\prime}-x_{1}^{\prime} & x_{3}^{\prime}-x_{1}^{\prime} \\
y_{2}^{\prime}-y_{1}^{\prime} & y_{3}^{\prime}-y_{1}^{\prime}
\end{array}\right] \text { and } \mathbf{b}_{(\triangle A D C)}=\left[\begin{array}{c}
x_{1}^{\prime} \\
y_{1}^{\prime}
\end{array}\right]
$$

For $\triangle A B C$ with vertices $A\left(x_{1}, y_{1}\right), C\left(x_{2}, y_{2}\right)$ and $B\left(x_{3}, y_{3}\right)$, we define following two matrices,

$$
\mathbf{F}_{(\triangle A B C)}=\left[\begin{array}{ll}
x_{2}-x_{1} & x_{3}-x_{1} \\
y_{2}-y_{1} & y_{3}-y_{1}
\end{array}\right] \text { and } \mathbf{b}_{(\triangle A B C)}=\left[\begin{array}{l}
x_{1} \\
y_{1}
\end{array}\right]
$$

After the affine transformation map, if the point $P^{\prime}\left(x^{\prime}, y^{\prime}\right)$ on the edge $A C_{(\triangle A D C)}$ is mapped at point $P(x, y)$ on the edge $A C_{(\triangle A B C)}$ then,

$$
\left[\begin{array}{l}
x \\
y
\end{array}\right]=\mathbf{F}_{(\triangle A B C)} \mathbf{F}_{(\triangle A D C)}^{-1}\left(P^{\prime}\right)+\left[\mathbf{b}_{(\triangle A B C)}-\mathbf{F}_{(\triangle A B C)} \mathbf{F}_{(\triangle A D C)}^{-1}\left(\mathbf{b}_{(\triangle A D C)}\right)\right]
$$

Using Eqn. A.24, we can map any point at the edge $A C_{(\triangle A D C)}$ affine to the edge $A C_{(\triangle A B C)}$.

\section{Homogeneously distributed points within a triangle}

For a given triangle $\triangle A B C$ with vertices $A \rightarrow\left(x_{1}, y_{1}\right), B \rightarrow\left(x_{2}, y_{2}\right)$ and $C \rightarrow\left(x_{3}, y_{3}\right)$, we generate a homogeneously distributed point $P(x, y)$ within this triangle, through a combination of the vertices $(A, B, C)$ and a pair of uniformly distributed random numbers $\left(r_{1}[0,1], r_{2}[0,1]\right)$ as described in (Osada et al., 2002),

$$
\vec{P}=\left(1-\sqrt{r_{1}}\right) \vec{A}+\sqrt{r_{1}}\left(1-r_{2}\right) \vec{B}+\sqrt{r_{1}} r_{2} \vec{C}
$$

Exact coordinate of $P(x, y)$ can be calculated as,

$$
\begin{aligned}
& x=\left(1-\sqrt{r_{1}}\right) x_{1}+\sqrt{r_{1}}\left(1-r_{2}\right) x_{2}+\sqrt{r_{1}} r_{2} x_{3} \\
& y=\left(1-\sqrt{r_{1}}\right) y_{1}+\sqrt{r_{1}}\left(1-r_{2}\right) y_{2}+\sqrt{r_{1}} r_{2} y_{3}
\end{aligned}
$$

\section{Intersection point of two line segments}

To find the intersection point of the line segments $\overline{P_{0} P_{1}}$ and $\overline{Q_{0} Q_{1}}$, we define three vectors $\hat{u}=\vec{P}_{1}-\vec{P}_{0}, \hat{v}=\vec{Q}_{1}-\vec{Q}_{0}, \hat{w}=\vec{P}_{0}-\vec{Q}_{0}$. We also define a vector $\hat{v}(s)=\hat{w}+s \hat{u}$ along the direction of $\hat{v}$, parametrised by a 
scalar parameter $s$. If the line segments $\overline{P_{0} P_{1}}$ and $\overline{Q_{0} Q_{1}}$ intersect at point $I=P\left(s_{I}\right)$, then at that point,

$$
\hat{v}\left(s_{I}\right)=\hat{w}+s_{I} \hat{u} .
$$

As $\hat{v}\left(s_{I}\right)$ is parallel to $\hat{v}$, we find the value of the scalar parameter, $s=s_{I}$ at the intersection point as follows,

$$
\hat{v}\left(s_{I}\right) \| \hat{v} \rightarrow \hat{v}\left(s_{I}\right) \cdot \hat{v}^{\perp}=0 \rightarrow\left(\hat{w}+s_{I} \hat{u}\right) \cdot \hat{v}^{\perp}=0 \rightarrow s_{I}=-\frac{\hat{v}^{\perp} \cdot \hat{w}}{\hat{v}^{\perp} \cdot \hat{u}}
$$

We can use, the pair of points $\left(P_{0}, P_{1}\right)$ and $s_{I}$ to calculate the intersection point between the line segments $\overline{P_{0} P_{1}}$ and $\overline{Q_{0} Q_{1}}$ as,

$$
\vec{I}=\vec{P}_{0}+s_{I}\left(\vec{P}_{1}-\vec{P}_{0}\right)
$$

\section{Local distribution of edge-catastrophes}

At a given edge (domain of high curvature) between two adjacent faces, the bending angle $\theta_{B}$ (see Fig. A.7, (B)) of a growing microtubule across the edge is determined by the value of edge-angle $\left(\theta_{E d g}^{a v g}\right.$, (see Fig. A.7, $(\mathrm{A})$ ) and the direction of the microtubule growth. Suppose a microtubule is bending through the edge $E$ when it makes a transition from the face $F_{1}$ to the face $F_{2}$. We draw a curve $C$ through the mean curvature domain of this edge $E$. Now, we define a unit vector $\hat{m}_{c}$ along the direction of the microtubule growth and a unit tangent vector $\hat{t}_{c}$ to $C$ at the point, where the microtubule transits from $F_{1}$ to $F_{2}$ (see Fig. A.7). Based on $\hat{m}_{c}$ and $\hat{t}_{c}$, we also define a smoothing angle,

$$
\theta_{s}=\cos ^{-1}\left(\hat{m}_{c} \cdot \hat{t}_{c}\right)
$$

We call $\theta_{s}$ to be smoothing angle, as depending on the value of this angle, the actual bending angle will be smooth (for $\theta_{s}=0$ ) or harsh (for $\theta_{s}=\frac{\pi}{2}$ ). Then, in terms of $\theta_{s}$ and $\theta_{E d g}^{a v g}$, we express the bending angle for the microtubule at this edge as,

$$
\theta_{B}=f\left(\theta_{s}\right) \theta_{E d g}^{a v g}
$$

where, $f\left(\theta_{s}\right)$ is a smoothing function. The maximum achievable value of the bending angle is $\theta_{B}^{\max }=\theta_{E d g}^{a v g}$, which is true at $\theta_{s}=\frac{\pi}{2}$ and the minimum achievable value of the bending angle is $\theta_{B}^{\min }=0$, which is true at $\theta_{s}=0$. This implies,

$$
\begin{aligned}
f\left(\theta_{s}\right) & =1, \text { at } \theta_{s}=\frac{\pi}{2} \\
& =0, \text { at } \theta_{s}=0
\end{aligned}
$$



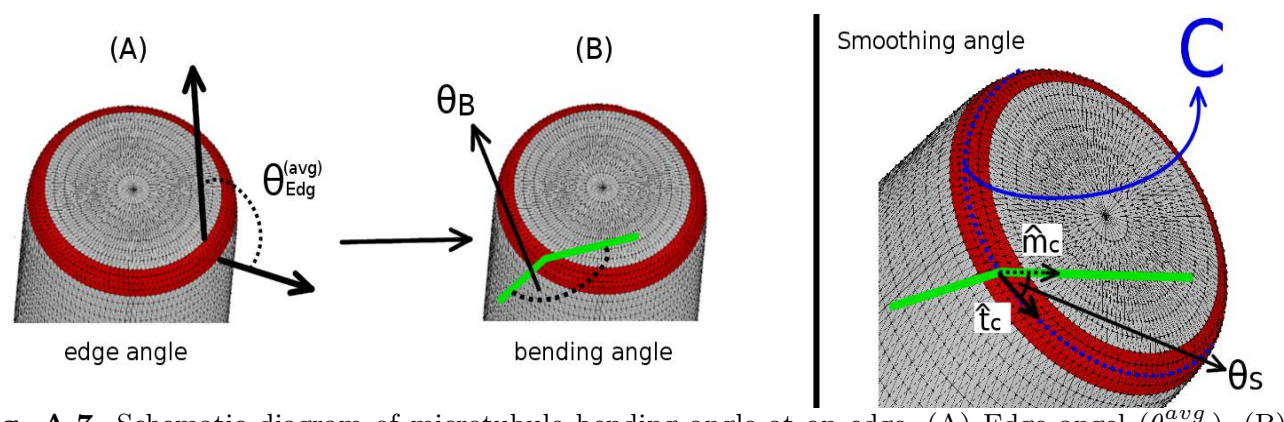

Fig. A.7. Schematic diagram of microtubule bending angle at an edge. (A) Edge angel $\left(\theta_{E d g}^{a v g}\right),(B)$ Bending angle $\left(\theta_{B}\right)$ and $(C)$ Smoothing angle $\theta_{s}$, which is an angle between the direction of microtubule growth $\left(\hat{m}_{c}\right)$ and the tangent $\left(\hat{t}_{c}\right)$ to the curve $C$ at the point of the microtubule crossing, along the mean curvature domain at the edge.

Any form of $f\left(\theta_{s}\right)$ that satisfies Eqn. A.29 and interpolate in the range $0 \leqslant \theta_{s} \leqslant \frac{\pi}{2}$, will be an acceptable choice of the smoothing function. For our simulation we use,

$$
f\left(\theta_{s}\right)=\sin ^{2} \theta_{s}
$$

Combining Eqn. A.28 and Eqn. A.30 we get,

$$
\theta_{B}=\theta_{E d g}^{a v g} \sin ^{2} \theta_{s}
$$

Experimental observations have shown that at an edge, the probability of edge catastrophe $\left(P_{E d g}\right)$ is roughly proportional to the bending angle $\left(\theta_{B}\right)$, i.e

$$
P_{E d g} \sim \theta_{B}
$$

However, given the experimental challenge in finding the exact relation, we formulate the following working relation,

$$
P_{E d g}=\frac{E_{c a t}}{2}\left(1-\cos \left(\theta_{B}\right)\right)
$$

where $0 \leqslant E_{\text {cat }} \leqslant 2$, is an edge-catastrophe multiplier, specific to a certain edge and its value depends on the biomechanical properties of that edge. In elongating root cells, on a smooth domain of cortex $\left(\theta_{B} \approx 0\right)$ microtubules undergo less edge-catastrophe. At a sharp edge with a value of $\theta_{B} \approx \frac{\pi}{2}$, microtubules are already prohibited from crossing that edge, i.e receive full edge-catastrophe $\left(P_{E d g}=1\right)$. We mathematically describe this as,

$$
\begin{aligned}
P_{E d g} & =0 \text { at } \theta_{B}=0 \\
& =1 \text { at } \theta_{B}=\frac{\pi}{2}
\end{aligned}
$$

From Eqn. A.32 and Eqn. A.33, we find that our definition of $P_{E d g}$ can be implemented in elongating root cells with $E_{c a t}=2$. Importing the 
expression of $\theta_{B}$ (see Eqn. A.31) into the Eqn. A.32, we end up with the following expression of the probability of edge catastrophe,

$$
\begin{aligned}
P_{E d g} & =\frac{E_{c a t}}{2}\left(1-\cos \left(\theta_{E d g}^{a v g} \sin ^{2} \theta_{s}\right)\right) \\
& =\frac{E_{c a t}}{2}\left(1-\cos \left(\theta_{E d g}^{a v g}\left(1-\cos ^{2} \theta_{s}\right)\right)\right)
\end{aligned}
$$

Using the value of $\theta_{s}$ (see Eqn. A.27) in Eqn. A.34 we get,

$$
P_{E d g}=\frac{E_{c a t}}{2}\left(1-\cos \left(\theta_{E d g}^{a v g}\left(1-\left(\hat{m}_{c} \cdot \hat{t}_{c}\right)^{2}\right)\right)\right)
$$

Now, using the local edge information of each pair of triangles $\left(T_{i}, T_{j}\right)$, i.e. $\theta_{e d g}^{\left(T_{i}, T_{j}\right)}$ (Eqn. A.18) and $w_{e d q}^{(i, j)}$ (Eqn. A.19), we can distribute the total edge-catastrophe $P_{E d g}$ (Eqn A.35) among the triangle-pair as,

$$
\begin{aligned}
p_{e d g}^{(i, j)} & =\frac{E_{c a t} w_{e d g}^{(i, j)}}{2}\left(1-\cos \left(\cos ^{-1}\left(\hat{n}_{i} \cdot \hat{n}_{j}\right)\left(1-\left(\hat{m}_{(i, j)} \cdot \hat{t}_{(i, j)}\right)^{2}\right)\right)\right) \\
& =\frac{E_{c a t} \cos ^{-1}\left(\hat{n}_{i} \cdot \hat{n}_{j}\right)}{2 \theta_{E d g}^{a v g}}\left(1-\cos \left(\cos ^{-1}\left(\hat{n}_{i} \cdot \hat{n}_{j}\right)\left(1-\left(\hat{m}_{(i, j)} \cdot \hat{t}_{(i, j)}\right)^{2}\right)\right)\right)
\end{aligned}
$$

where, $\hat{m}_{(i, j)}$ is the growth direction of a microtubule passing from $T_{i}$ to $T_{j}$ and $\hat{t}_{(i, j)}$ is a unit vector along the shared edge between $T_{i}$ and $T_{j} . \hat{n}_{i}$ and $\hat{n}_{j}$ are normals to $T_{i}$ and $T_{j}$ respectively, and both pointing either inwards or outwards to the triangulated surface mesh.

\section{A.3 Order parameter}

Consider a (piecewise) smooth oriented 2-manifold $M$ embedded in $\mathbb{R}^{3}$, which we call the surface. Except for possibly a set of areal measure zero, we can endow a tangent space at each of its points $\sigma$ with an orthonormal reference frame defined by two unit vectors $\hat{e}_{1}(\sigma)$ and $\hat{e}_{2}(\sigma)$, using the prescription that the outward normal to the surface is given by,

$$
\hat{n}(\sigma)=\hat{e}_{1}(\sigma) \times \hat{e}_{2}(\sigma)
$$

At this local tangent pane, the orientation of a particle at $\sigma$ can be described by a unit vector,

$$
\begin{aligned}
\hat{\omega} & =\omega_{1} \hat{e}_{1}(\sigma)+\omega_{2} \hat{e}_{2}(\sigma)+\omega_{3} \hat{n}(\sigma) \\
& =\omega_{1} \hat{e}_{1}(\sigma)+\omega_{2} \hat{e}_{2}(\sigma)+0 \hat{n}(\sigma) \\
& =\cos \alpha \hat{e}_{1}(\sigma)+\sin \alpha \hat{e}_{2}(\sigma)
\end{aligned}
$$

where $\alpha \in[0,2 \pi)$ is the orientation angle of $\hat{\omega}$ with respect to the direction of $\hat{e}_{1}(\sigma)$. 


\section{Local order parameter tensor}

The local order parameter tensor $\mathbf{q}^{(m)}(\sigma)$ at $\sigma$ is $O(2)$ irreducible tensor associated with rotations around $\hat{n}(\sigma)$, but now interpreted as tensors embedded in the ambient 3-space as,

$$
\mathbf{q}^{(m)}(\sigma)=q_{i_{1} i_{2} \ldots i_{m}}^{(m)}(\sigma) \bigotimes_{k=1, m} \hat{\mathbf{e}}_{i_{k}}(\sigma)
$$

where $\otimes$ denotes direct product.

We follow the convention that lower in italic indices take on the values $\{1,2\}$ and we adopt Einstein summation convention. Components of the tensor $\mathbf{q}^{(m)}(\sigma)$ upto order two are explicitly given by ,

$$
\begin{aligned}
& q^{(0)}(\sigma)=\langle 1\rangle_{\sigma} \\
& q_{i}^{(1)}(\sigma)=\left\langle\omega_{i}\right\rangle_{\sigma} \\
& q_{i j}^{(2)}(\sigma)=2\left\langle\omega_{i} \omega_{j}\right\rangle_{\sigma}-\delta_{i j}
\end{aligned}
$$

where $\langle\ldots\rangle_{\sigma}$ denotes orientational average, i.e. if distribution of possible orientations at $\sigma$ is coded into a normalized orientational distribution $\psi_{\sigma}(\alpha)$, then the orientational average is,

$$
\langle f\rangle_{\sigma}=\int_{0}^{2 \pi} d \alpha f(\alpha) \psi_{\sigma}(\alpha)
$$

On the local tangent space, the tensor components are symmetric and traceless for $m \geq 2$.

For example

$$
q_{i j}^{(2)}=\left[\begin{array}{cc}
2 \cos ^{2} \alpha-1 & 2 \cos \alpha \sin \alpha \\
2 \cos \alpha \sin \alpha & 2 \sin ^{2} \alpha-1
\end{array}\right]=\left[\begin{array}{cc}
\cos 2 \alpha & \sin 2 \alpha \\
\sin 2 \alpha & -\cos 2 \alpha
\end{array}\right]
$$

we choose the normalization such that,

$$
q_{11 \cdots 1}^{(m)}(\sigma)=\langle\cos m \alpha\rangle_{\sigma}
$$

\section{Global order parameter tensor}

We can average the local order parameter tensors over the full surface to extract the global order parameter tensor,

$$
\mathbf{Q}^{(m)}=\frac{\int d A(\sigma) \rho(\sigma) \mathbf{q}^{(m)}(\sigma)}{\int d A(\sigma) \rho(\sigma)}
$$

where $\rho(\sigma)$ is the local areal density of the orientable constituents present on the elementary area $\mathrm{dA}(\sigma)$. For $m \geq 2$, symmetric and traceless properties 
of $\mathbf{q}^{(m)}$ remain conserved by the surface averaging process, i.e $\mathbf{Q}^{(m)}$ is also symmetric and traceless.

Consider a triangulated three dimensional surface, where a bunch of rod like particles (line segments) are distributed over each triangle of the three dimensional surface. For a triangle $E$, at its center we assign a tangent plane with an orthonormal reference frame defined by the unit vectors $\hat{e}_{1}, \hat{e}_{2}$ and $\hat{n}=\hat{e}_{1} \times \hat{e}_{2}$. Lets assume on the plane of this triangle, a particle of length $l_{i}(E)$ is oriented with angle $\theta_{i}$, with respect to the direction of $\hat{e}_{1}$. In the local tangent frame, corresponding orientation vector (Eqn. A.36) of this particle is,

$$
\begin{aligned}
\hat{\omega}^{i} & =\omega_{1}^{i} \hat{e}_{1}+\omega_{2}^{i} \hat{e}_{2}+\omega_{3}^{i} \hat{n} \\
& =\cos \theta_{i} \hat{e}_{1}+\sin \theta_{i} \hat{e}_{2}+0 \hat{n} \\
& =\cos \theta_{i} \hat{e}_{1}+\sin \theta_{i} \hat{e}_{2}
\end{aligned}
$$

In matrix form,

$$
\omega^{i}=\left[\begin{array}{c}
\omega_{1}^{i} \\
\omega_{2}^{i} \\
\omega_{3}^{i}
\end{array}\right]=\left[\begin{array}{c}
\cos \theta_{i} \\
\sin \theta_{i} \\
0
\end{array}\right]
$$

If $\mathbf{q}^{(2)}(E)$ is the local order parameter tensor associated with the triangle $E$, then the associated tensor components (Eqn. A.37) are,

$$
\begin{aligned}
& q_{11}^{(2)}(E)=\frac{2 \sum_{i=1}^{l_{\max }} l_{i}(E) \omega_{1}^{i} \omega_{1}^{i}}{\sum_{i=1}^{l_{\max }} l_{i}(E)}-1 \\
& q_{22}^{(2)}(E)=\frac{2 \sum_{i=1}^{l_{\max }} l_{i}(E) \omega_{2}^{i} \omega_{2}^{i}}{\sum_{i=1}^{l_{\max }} l_{i}(E)}-1 \\
& q_{33}^{(2)}(E)=\frac{2 \sum_{i=1}^{l_{\max }} l_{i}(E) \omega_{3}^{i} \omega_{3}^{i}}{\sum_{i=1}^{l_{\max }} l_{i}(E)}-1=0 \\
& q_{12}^{(2)}(E)=\frac{2 \sum_{i=1}^{l_{\max }} l_{i}(E) \omega_{1}^{i} \omega_{2}^{i}}{\sum_{i=1}^{l_{\max }} l_{i}(E)}-1=q_{21}^{(2)}(E) \\
& q_{13}^{(2)}(E)=\frac{2 \sum_{i=1}^{l_{\max }} l_{i}(E) \omega_{1}^{i} \omega_{3}^{i}}{\sum_{i=1}^{l_{\max }} l_{i}(E)}-1=q_{31}^{(2)}(E)=0 \\
& q_{23}^{(2)}(E)=\frac{2 \sum_{i=1}^{l_{\max }} l_{i}(E) \omega_{2}^{i} \omega_{3}^{i}}{\sum_{i=1}^{l_{\max }} l_{i}(E)}-1=q_{32}^{(2)}(E)=0
\end{aligned}
$$

where $l_{\max }$ is the total number of particles present in the triangle $E$. 
In matrix form,

$$
\mathbf{q}^{(2)}(E)=\left[\begin{array}{lll}
q_{11}^{(2)}(E) & q_{12}^{(2)}(E) & q_{13}^{(2)}(E) \\
q_{12}^{(2)}(E) & q_{22}^{(2)}(E) & q_{23}^{(2)}(E) \\
q_{13}^{(2)}(E) & q_{23}^{(2)}(E) & q_{33}^{(2)}(E)
\end{array}\right]=\left[\begin{array}{ccc}
q_{11}^{(2)}(E) & q_{12}^{(2)}(E) & 0 \\
q_{12}^{(2)}(E) & q_{22}^{(2)}(E) & 0 \\
0 & 0 & 0
\end{array}\right]
$$

This local order parameter tensor $\mathbf{q}^{(2)}(E)$ is defined at the local tangent frame attached to the triangle $E$. To express this tensor with respect to the global coordinate frame, first we express the basis vectors $\left(\hat{e}_{1}, \hat{e}_{2}, \hat{n}=\hat{e}_{1} \times \hat{e}_{2}\right)$ of the local tangent frame with respect to the global frame $(\hat{x}, \hat{y}, \hat{z})$ as,

$$
\begin{aligned}
& \hat{e}_{1}=e_{1}^{(x)} \hat{x}+e_{1}^{(y)} \hat{y}+e_{1}^{(z)} \hat{z} \\
& \hat{e}_{2}=e_{2}^{(x)} \hat{x}+e_{2}^{(y)} \hat{y}+e_{2}^{(z)} \hat{z} \\
& \hat{n}=n^{(x)} \hat{x}+n^{(y)} \hat{y}+n^{(z)} \hat{z}
\end{aligned}
$$

In matrix form,

$$
e_{1}=\left[\begin{array}{l}
e_{1}^{(x)} \\
e_{1}^{(y)} \\
e_{1}^{(z)}
\end{array}\right], e_{2}=\left[\begin{array}{l}
e_{2}^{(x)} \\
e_{2}^{(y)} \\
e_{2}^{(z)}
\end{array}\right] \text { and } n=\left[\begin{array}{l}
n^{(x)} \\
n^{(y)} \\
n^{(z)}
\end{array}\right]
$$

Now, we define a rotation matrix $P$ between the local tangent frame and the global frame,

$$
P=\left[\begin{array}{lll}
e_{1}^{(x)} & e_{2}^{(x)} & n^{(x)} \\
e_{1}^{(y)} & e_{2}^{(y)} & n^{(y)} \\
e_{1}^{(z)} & e_{2}^{(z)} & n^{(z)}
\end{array}\right]
$$

Using the rotational transformation, we express the local order parameter tensor $\mathbf{q}^{(2)}(E)$ with respect to the global coordinate frame as,

$$
\mathbf{q}_{r}^{(2)}(E)=P \mathbf{q}^{(2)}(E) P^{T}
$$

For all the triangles of the three dimensional polyhedral surface, averaging over the local order parameter tensors $\mathbf{q}_{r}{ }^{(2)}$, we will get the global order parameter tensor (Eqn. A.38),

$$
\mathbf{Q}^{(2)}=\frac{\sum_{j=1}^{N} L_{j} \mathbf{q}_{r}^{(2)}\left(E_{j}\right)}{\sum_{j=1}^{N} L_{j}}
$$

where $L_{j}$ is length of all the particles present on the triangle $E_{j}$ and $N$ is the total number of triangle used to approximate the polyhedral surface. 


\section{References}

Aida, M., Beis, D., Heidstra, R., Willemsen, V., Blilou, I., Galinha, C., Nussaume, L., Noh, Y. S., Amasino, R., and Scheres, B. (2004). The plethora genes mediate patterning of the arabidopsis root stem cell niche. Cell, 119:109.

Allard, J. F., Wasteneys, G. O., and Cytrynbaum, E. N. (2010). Mechanisms of self-organization of cortical microtubules in plants revealed by computational simulations. Molecular Biology of the Cell, 21:278.

Ambrose, C., Allard, J. F., Cytrynbaum, E. N., and Wasteneys, G. O. (2011). A clasp-modulated cell edge barrier mechanism drives cell-wide cortical microtubule organization in arabidopsis. Nat. Com., 2:430.

Ambrose, J. C., Shoji, T., Kotzer, A. M., Pighin, J. A., and Wasteneys, G. O. (2007). The arabidopsis clasp gene encodes a microtubule-associated protein involved in cell expansion and division. Plant Cell, 19:2763.

Ambrose, J. C. and Wasteneys, G. O. (2008). Clasp modulates microtubulecortex interaction during self-organization of acentrosomal microtubules. Mol. Biol. Cell., 19:4730.

Asada, T. and Collings, D. (1997). Molecular motors in higher plants. Trends Plant Sci., 2:29.

Baskin, T. I. (2001). On the alignment of cellulose microfibrils by cortical microtubules: A review and a model. Protoplasma, 215:150.

Baskin, T. I., Beemster, G. T., Judy-March, J. E., and Marga, F. (2004). Disorganization of cortical microtubules stimulates tangential expansion and reduces the uniformity of cellulose microfibril alignment among cells in the root of arabidopsis. Plant Physiol., 135:2279.

Baulin, V. A., Marques, C. M., and Thalmann, F. (2007). Collision induced spatial organization of microtubules. Biophys. Chem., 128:231.

Besson, S. and Dumais, J. (2011). Universal rule for the symmetric division of plant cells. Proc. Natl. Acad. Sci. USA, 108:6294.

Boer, D. R., Freire-Rios, A., van den Berg, W. A., Saaki, T., Manfield, I. W., Kepinski, S., Lòpez-Vidrieo, I., Franco-Zorrilla, J. M., de Vries, S. C., Solano, R., Weijers, D., and Coll, M. (2014). Structural basis for dna binding specificity by the auxin-dependent arf transcription factors. Cell, 156:577. 
Bringmann, M., Li, E., Sampathkumar, A., T., K., Hauser, M. T., and Persson, S. (2012). Pom-pom2/cellulose synthase interacting1 is essential for the functional association of cellulose synthase and microtubules in arabidopsis. Plant Cell, 24:163.

Burian, A., Ludynia, M., Uyttewaal, M., Traas, J., Boudaoud, A., Hamant, O., and Kwiatkowska, D. (2013). A correlative microscopy approach relates microtubule behaviour, local organ geometry, and cell growth at the arabidopsis shoot apical meristem. J. Exp. Bot., 64:5753.

Burk, D. H. and Ye, Z. H. (2001). Alteration of oriented deposition of cellulose microfibrils by mutation of a katanin-like microtubule-severing protein. Plant Cell, 14:2145.

Campilho, A., Garcia, B., v.d. Toorn, H., v. Wijk, H., Campilho, A., and Scheres, B. (2006). Time-lapse analysis of stem-cell divisions in the arabidopsis thaliana root meristem. The Plant Journal, 48:619.

Chan, J., Calder, G. M., Doonan, J. H., and Lloyd, C. W. (2003). Eb1 reveals mobile microtubule nucleation sites in arabidopsis. Nature Cell Biol., 5:967.

Chan, J., Sambade, A., Calder, G., and Lloyd, C. (2009). Arabidopsis cortical microtubules are initiated along, as well as branching from, existing microtubules. Plant Cell, 21:2298.

Cignoni, P., Callieri, M., Corsini, M., Dellepiane, M., Ganovelli, F., and Ranzuglia, G. (2008). MeshLab: an Open-Source Mesh Processing Tool. In Scarano, V., Chiara, R. D., and Erra, U., editors, Eurographics Italian Chapter Conference. The Eurographics Association.

Clark, J. K. and Sheridan, W. F. (1988). Characterization of the two maize embryo-lethal defective kernel mutants rgh*-1210 andfl*-1253b: Effects on embryo and gametophyte development. Genetics, 120:279.

Cleary, A. L. (2001). Plasma membrane-cell wall connections: roles in mitosis and cytokinesis revealed by plasmolysis of tradescantia virginiana leaf epidermal cells. Protoplasma, 215:21.

Cleary, A. L., Gunning, B. E. S., Wasteneys, G., and Hepler, P. (1992). Microtubule and f-actin dynamics at the division site in living tradescantia stamen hair cells. J. Cell Sci., 103:977.

Costa, L. M., Gutierrez-Marcos, J. F., Brutnell, T. P., Greenland, A. J., and Dickinson, H. G. (2003). The globby1-1 (glo1-1) mutation disrupts nuclear and cell division in the developing maize seed causing alterations in endosperm cell fate and tissue differentiation. Development, 130:5009. 
Crowell, E. F., Bischoff, V., Desprez, T., Rolland, A., Stierhof, Y. D., Schumacher, K., Gonneau, M., Höfte, H., and Vernhettes, S. (2009). Pausing of golgi bodies on microtubules regulates secretion of cellulose synthase complexes in arabidopsis. Plant Cell, 21:1141.

Davidson, E. (2006). The regulatory genome. Elsevier.

de Reuille, P. B., Routier-Kierzkowska, A. L., Kierzkowski, D., Bassel, G. W., Schüpbach, T., Tauriello, G., Bajpai, N., Strauss, S., Weber, A., Kiss, A., Burian, A., Hofhuis, H., Sapala, A., Lipowczan, M., Heimlicher, M. B., Robinson, S., Bayer, E. M., Basler, K., Koumoutsakos, P., Roeder, A. H., Aegerter-Wilmsen, T., Nakayama, N., Tsiantis, M., Hay, A., Kwiatkowska, D., Xenarios, I., Kuhlemeier, C., and Smith, R. S. (2015). Morphographx: A platform for quantifying morphogenesis in 4d. eLife., 4:e05864.

Deinum, E. E., Tindemans, S. H., and Mulder, B. M. (2011). Taking directions: the role of microtubule-bound nucleation in the self-organization of the plant cortical array. Phys. Biol., 8:056002.

Dharmasiri, N., Dharmasiri, S., and Estelle, M. (2005). The f-box protein tir1 is an auxin receptor. Nature, 435:441.

Dhonukshe, P. and Gadella, T. W. J. (2003). Alteration of microtubule dynamic instability during preprophase band formation revealed by yellow fluorescent protein-clip170 microtubule plus-end labeling. Plant Cell, 15:597.

Dhonukshe, P., Laxalt, A. M., Goedhart, J., Gadella, T. W., and Munnik, T. (2003). Phospholipase d activation correlates with microtubule reorganization in living plant cells. Plant Cell, 15:2666.

Dixit, R. and Cyr, R. (2004). Encounters between dynamic cortical microtubules promote ordering of the cortical array through angle-dependent modifications of microtubule behavior. The Plant Cell, 16:3274.

Dixit, R. and Cyr, R. J. (2002). Spatio-temporal relationship between nuclearenvelope breakdown and preprophase band disappearance in cultured tobacco cells. Protoplasma, 219:116.

Dolan, L., Janmaat, K., Willemsen, V., Linstead, P., Poethig, S., Roberts, K., and Scheres, B. (1993). Cellular organisation of the arabidopsis thaliana root. Development, 119:71.

Dráber, P., Sulimenko, V., and Dráberová, E. (2012). Cytoskeleton in mast cell signaling. Front Immunol., 3:130. 
Dupuy, L., Mackenzie, J., and Haseloff, J. (2010). Coordination of plant cell division and expansion in a simple morphogenetic system. Proc. Natl. Acad. Sci. USA, 107:2711.

Ehrhardt, D. W. and Shaw, S. L. (2006). Microtubule dynamics and organization in the plant cortical array. Annu. Rev. Plant Biol., 57:859.

Emons, A. M. C., Höfte, H., and Mulder, B. M. (2007). Microtubules and cellulose microfibrils: how intimate is their relationship ? Trends in Plant Science, 12:279.

Eren, E. C., Dixit, R., and Gautam, N. (2010). A three-dimensional computer simulation model reveals the mechanisms for self-organization of plant cortical microtubules into oblique arrays. Molecular Biology of the Cell, 21:2674.

Errera, L. (1888). Überzellformen und seifenblasen. Bot. Zentralbl., 34:395.

Fichthorn, K. A. and Weinberg, W. H. (1991). Theoretical foundations of dynamical monte carlo simulations. J. Chem. Phys., 95:1090.

Galinha, C., Hofhuis, H., Luijten, M., Willemsen, V., Blilou, I., Heidstra, R., and Scheres, B. (2007). Plethora proteins as dose-dependent master regulators of arabidopsis root development. Nature, 449:1053.

Gardiner, J. C., Harper, J. D., Weerakoon, N. D., Collings, D. A., Ritchie, S., Gilroy, S., Cyr, R. J., and Marc, J. (2001). A 90-kd phospholipase d from tobacco binds to microtubules and the plasma membrane. Plant Cell, 13:2143.

Gillespie, D. T. (1977). Exact stochastic simulation of coupled chemical reactions. J. Phys. Chem., 81:2340.

Gomez, J. M., Chumakova, L., Bulgakova, N. A., and Brown, N. H. (2016). Microtubule organization is determined by the shape of epithelial cells. Nat. Com., 7:13172.

Granger, C. L. and Cyr, R. J. (2000). Microtubule reorganization in tobacco by-2 cells stably expressing gfp-mbd. Planta, 210:502.

Green, P. B. (1980). Organogenesis: A biophysical view. Annu. Rev. Plant Physiol., 31:51.

Grego, S., Cantillana, V., and Salmon, E. D. (2001). Microtubule treadmilling in vitro investigated by fluorescence speckle and confocal microscopy. Biophysical Journal, 81:66. 
Guilfoyle, T. J. and Hagen, G. (2007). Auxin response factors. Current Opinion in Plant Biology, b10:453.

Hamada, T. (2007). Microtubule-associated proteins in higher plants. J. Plant Res., 120:79.

Hamann, T., Benkova, E., Bäurle, I., Kientz, M., and Jürgens, G. (2002). The arabidopsis bodenlos gene encodes an auxin response protein inhibiting monopteros-mediated embryo patterning. Genes Dev., 16:1610.

Hamann, T., Mayer, U., and Jürgens, G. (1999). The auxin-insensitive bodenlos mutation affects primary root formation and apical-basal patterning in the arabidopsis embryo. Development, 126:1387.

Hamant, O., Heisler, M. G., Jönsson, H., Krupinski, P., Uyttewaal, M., Bokov, P., Corson, F., Sahlin, P., Boudaoud, A., Meyerowitz, E. M., Couder, Y., and Traas, J. (2008). Developmental patterning by mechanical signals in arabidopsis. Science, 322:1650.

Hardham, A. R. and Gunning, B. E. (1978). Structure of cortical microtubule arrays in plant cells. J. Cell Biol., 77:14.

Hardtke, C. S. and Berleth, T. (1998). The arabidopsis gene monopteros encodes a transcription factor mediating embryo axis formation and vascular development. EMBO J., 17:1405.

Harris, D. M., Corbin, K., Wang, T., Gutierrez, R., Bertolo, A. L., Petti, C., Smilgies, D. M., Estevez, J. M., Bonetta, D., Urbanowicz, B. R., Ehrhardt, D. W., Somerville, C. R., Rose, J. K. C., Hong, M., and DeBolt, S. (2012). Cellulose microfibril crystallinity is reduced by mutating cterminal transmembrane region residues cesa1 a903v and cesa3 t942i of cellulose synthase. Proc. Natl. Acad. Sci. USA, 109:4098.

Hashimoto, T. and Kato, T. (2006). Cortical control of plant microtubules. Curr. Opin. Plant. Biol., 9:5.

Hawkins, R. J., Tindemans, S. H., and Mulder, B. M. (2010). Model for the orientational ordering of the plant microtubule cortical array. Phy. Rev. E, 82:011911.

Heisler, M. G., Hamant, O., Krupinski, P., Uyttewaal, M., C. Ohno, H. J., Traas, J., and Meyerowitz, E. M. (2010). Alignment between pin1 polarity and microtubule orientation in the shoot apical meristem reveals a tight coupling between morphogenesis and auxin transport. PLoS Biol., 8:e1000516. 
Himmelspach, R., Williamson, R. E., and Wasteneys, G. O. (2003). Cellulose microfibril alignment recovers from dcb-induced disruption despite microtubule disorganization. Plant J., 36:565.

Hofmeister, W. (1863). Zusätze und berichtigungen zu den 1851 veröffentlichten untersuchungen der entwicklung hoherer höherer krytogamen. Jahrb. Wiss. Bot., 3:259.

Hush, J. M., Wadsworth, P., Callaham, D. A., and Hepler, P. K. (1994). Quantification of microtubule dynamics in living plant cells using fluorescence redistribution after photobleaching. J. Cell Sci., 107:775.

Jaspersen, S. L. and Winey, M. (2004). The budding yeast spindle pole body:structure, duplication, and function. Annu. Rev. Cell Dev. Biol., $20: 1$.

Jürgens, G. (1995). Axis formation in plant embryogenesis: Cues and clues. Cell, 81:467.

Jürgens, G. (2001). Apical-basal pattern formation in arabidopsis embryogenesis. EMBO J., 20:3609.

Kepinski, S. and Leyser, O. (2005). The arabidopsis f-box protein tir1 is an auxin receptor. Nature, 435:446.

Kimata, Y., Higaki, T., Kawashima, T., Kurihara, D., Sato, Y., Yamada, T., Hasezawa, S., Berger, F., Higashiyama, T., and Ueda, M. (2016). Cytoskeleton dynamics control the first asymmetric cell division in arabidopsis zygote. Proc. Natl. Acad. Sci. USA, 113:14157.

Kirik, V., Herrmann, U., Parupalli, C., Sedbrook, J. C., Ehrhardt, D. W., and Hülskamp, M. (2007). Clasp localizes in two discrete patterns on cortical microtubules and is required for cell morphogenesis and cell division in arabidopsis. J. Cell. Sci., 120:4416.

Kumagai, F., Yoneda, A., Tomida, T., Sano, T., Nagata, T., and Hasezawa, S. (2001). Fate of nascent microtubules organized at the $\mathrm{m} / \mathrm{g} 1$ interface, as visualized by synchronized tobacco by-2 cells stably expressing gfptubulin: time-sequence observations of the reorganization of cortical microtubules in living plant cells. Plant Cell Physiol., 42:723.

Kwiatkowska, D. (2004). Structural integration at the shoot apical meristem: models, measurements, and experiments. Am. J. Bot., 91:1277.

Landrein, B. and Hamant, O. (2013). How mechanical stress controls microtubule behavior and morphogenesis in plants: history, experiments and revisited theories. The Plant Journal, 75:324. 
Li, S. B., Xie, Z. Z., Hu, C. G., and Zhang, J. Z. (2016). A review of auxin response factors (arfs) in plants. Front. Plant Sci., 7:47.

Lindeboom, J., Mulder, B. M., Vos, J. W., Ketelaar, T., and Emons, A. M. (2008). Cellulose microfibril deposition: coordinated activity at the plant plasma membrane. J. Microsc., 231:192.

Lindeboom, J. J., Lioutas, A., Deinum, E. E., Tindemans, S. H., Ehrhardt, D. W., Emons, A. M. C., Vos, J. W., and Mulder, B. M. (2013). Cortical microtubule arrays are initiated from a nonrandom prepattern driven by atypical microtubule initiation. Plant Physiology, 161:1189.

Lloyd, C. and Buschmann, H. (2007). Plant division: Remembering where to build the wall. Current Biology, 17:R1053.

Lloyd, C. W. (2001). Plant Microtubules: Their Role in Growth and Development. John Wiley \& Sons, Ltd.

Louveaux, M., Julien, J. D., Mirabet, V., Boudaoud, A., and Hamant, O. (2016). Cell division plane orientation based on tensile stress in arabidopsis thaliana. Proc. Natl. Acad. Sci. USA, 113:E4294.

Lucas, J. and Shaw, S. L. (2008). Cortical microtubule arrays in the arabidopsis seedling. Current Opinion in Plant Biology, 11:94.

Lukowitz, W., Roeder, A., Parmenter, D., and Somerville, C. (2004). A mapkk kinase gene regulates extra-embryonic cell fate in arabidopsis. Cell, 116:109.

Magnard, J. L., Heckel, T., Massonneau, A., Wisniewski, J. P., Cordelier, S., Lassagne, H., Perez, P., Dumas, C., and Rogowsky, P. M. (2004). Morphogenesis of maize embryos requires zmprpl35-1 encoding a plastid ribosomal protein. Plant Physiol., 134:649.

Mähönen, A. P., Tusscher, K., Siligato, R., Smetana, O., Díaz-Triviño, S., Salojärvi, J., Wachsman, G., Prasad, K., Heidstra, R., and Scheres, B. (2014). Plethora gradient formation mechanism separates auxin responses. Nature, 515:125.

Mandelkow, E. and Mandelkow, E. M. (1994). Microtubule structure. Current opinion in Structural Biology, 4:171.

Matov, A., Applegate, K., Kumar, P., Thoma, C., Krek, W., Danuser, G., and Wittmann, T. (2010). Analysis of microtubule dynamic instability using a plus-end growth marker. Nature Methods, 7:761.

McCurdy, D. W., Kovar, D. R., and Staiger, C. J. (2001). Actin and actin-binding proteins in higher plants. Protoplasma, 215:89. 
Meinhardt, H. (1998). The Algorithmic beauty of sea shells. Springer-Verlag.

Mineyuki, Y. (1999). The preprophase band of microtubules: its function as a cytokinetic apparatus in higher plants. Int. Rev. Cytol., 187:1.

Molchan, T. M., Valster, A. H., and Hepler, P. (2002). Actomyosin promotes cell plate alignment and late lateral expansion in tradescantia stamen hair cells. Planta, 214:683.

Murata, T., Sonobe, S., Baskin, T. I., Hyodo, S., Hasezawa, S., Nagata, T., Horio, T., and Hasebe, M. (2005). Microtubule-dependent microtubule nucleation based on recruitment of big gamma-tubulin in higher plants. Nat. Cell Biol., 7:961.

Nakielski, J. (2000). Tensorial model for growth and cell division in the shoot apex. In Pattern Formation in Biology, Vision and Dynamics. World Scientific.

Osada, R., Funkhouser, T., Chazelle, B., and Dobkin, D. (2002). Shape distributions. ACM Trans. Graph., 21:807.

Panda, D., Miller, H. P., and Wilson, L. (1999). Rapid treadmilling of brain microtubules free of microtubule-associated proteins in vitro and its suppression by tau. Proc. Natl. Acad. Sci. USA, 96:12459.

Paredez., A. R., Persson., S., Ehrhardt, D. W., and Somerville, C. R. (2008). Genetic evidence that cellulose synthase activity influences microtubule cortical array organization. Plant Physiology, 147:1723.

Paredez, A. R., Somerville, C. R., and Ehrhardt, D. W. (2006). Visualization of cellulose synthase demonstrates functional association with microtubules. Science, 312:1491.

Phillips, A. R. and Evans, M. M. S. (2011). Analysis of stunter1, a maize mutant with reduced gametophyte size and maternal effects on seed development. Genetics, 187:1085.

Pickett-Heaps, J. D., Gunning, B. E. S., Brown, R. C., Lemmon, B. E., and Cleary, A. L. (1999). The cytoplast concept in dividing plant cells: cytoplasmic domains and the evolution of spatially organized cell division. Am. J. Bot., 86:153.

Pinon, V., Prasad, K., Grigg, S. P., Sanchez-Perez, G. F., and Scheres, B. (2013). Local auxin biosynthesis regulation by plethora transcription factors controls phyllotaxis in arabidopsis. Proc. Natl. Acad. Sci. USA, 110:1107. 
Rademacher, E. H., Lokerse, A. S., Schlereth, A., Llavata-Peris, C. I., Bayer, M., Kientz, M., Freire, A. R., Borst, J. W., Lukowitz, W., Jürgens, G., and Weijers, D. (2012). Different auxin response machineries control distinct cell fates in the early plant embryo. Dev. Cell, 22:211.

Reddy, A. S. (2001). Molecular motors and their functions in plants. Int Rev Cytol, 204:97.

Reddy, J. (2006). An Introduction to the Finite Element Method (Third ed.). McGraw-Hill.

Rodionov, V. I. and Borisy, G. G. (1997). Microtubule treadmilling in vivo. Science, 275:215.

Rothwell, S. W., Grasser, W. A., and Murphy, D. B. (1985). Direct observation of microtubule treadmilling by electron microscopy. The Journal of Cell Biology, 101:1637.

Ruegger, M., Dewey, E., Gray, W. M., Hobbie, L., Turner, J., and Estelle, M. (1998). The tir1 protein of arabidopsis functions in auxin response and is related to human skp2 and yeast grr1p. Genes Dev., 12:198.

Rybel, B. D., Möller, B., Yoshida, S., Grabowicz, I., de Reuille, P. B., Boeren, S., Smith, R. S., Borst, J. W., and Weijers, D. (2013). A bhlh complex controls embryonic vascular tissue establishment and indeterminate growth in arabidopsis. Developmental Cell, 24:426.

Sachs, J. (1878). Über die anordnung der zellen in jüngsten pflanzenteilen. Arb. Bot. Inst. Wur̈zburg, 2:46.

Sahlin, P. and Jönsson, H. (2010). A modeling study on how cell division affects properties of epithelial tissues under isotropic growth. PLoS One, $5: \mathrm{e} 11750$.

Sampathkumar, A., Krupinski, P., Wightman, R., Milani, P., Berqu, A., Boudaoud, A., Hamant, O., Jönsson, H., and Meyerowitz, E. M. (2014). Subcellular and supracellular mechanical stress prescribes cytoskeleton behavior in arabidopsis cotyledon pavement cells. eLife, 3:e01967.

Santuari, L., Sanchez-Perez, G. F., Luijten, M., Rutjens, B., Terpstra, I., Berke, L., Gorte, M., Prasad, K., Bao, D., Timmermans-Hereijgers, J. L., Maeo, K., Nakamura, K., Shimotohno, A., Pencik, A., Novak, O., Ljung, K., van Heesch, S., de Bruijn, E., Cuppen, E., Willemsen, V., Mähönen, A. P., Lukowitz, W., Snel, B., de Ridder, D., Scheres, B., and Heidstra, R. (2016). The plethora gene regulatory network guides growth and cell differentiation in arabidopsis roots. Plant Cell, 28:2937. 
Scheible, W. R., Eshed, R., Richmond, T., Delmer, D., and Somerville, C. (2001). Modifications of cellulose synthase confer resistance to isoxaben and thiazolidinone herbicides in arabidopsis ixr1 mutants. Proc. Natl. Acad. Sci. USA, 98:10079.

Scheres, B. and van der Putten, W. H. (2017). The plant perceptron connects environment to development. Nature, 543:11.

Schmit, A. C. (2002). A centrosomal microtubule nucleation in higher plants. Int. Rev. Cytol., 220:257.

Shaw, S. L., Kamyar, R., and Ehrhardt, D. W. (2003). Sustained microtubule treadmilling in arabidopsis cortical arrays. Science, 300:1715.

Shi, X. and Ma, Y. (2010). Understanding phase behaviour of plant cell cortex microtubule organization. Proc. Natl. Acad. Sci. USA, 107:11709.

Smet, S. D., Cuypers, A., Vangronsveld, J., and Remans, T. (2015). Gene networks involved in hormonal control of root development in arabidopsis thaliana: A framework for studying its disturbance by metal stress. Int. J. Mol. Sci., 16:19195.

Smith, R. S., Guyomarc'h, S., Mandel, T., Reinhardt, D., Kuhlemeier, C., and Prusinkiewicz, P. (2006). A plausible model of phyllotaxis. Proc. Natl. Acad. Sci. USA, 103:1301.

Staehelin, L. A. and Hepler, P. K. (1996). Cytokinesis in higher plants. Cell, 84:821.

Stoma, S., Lucas, M., Chopard, J., Schaedel, M., Traas, J., and Godin, C. (2008). Flux-based transport enhancement as a plausible unifying mechanism for auxin transport in meristem development. PLoS Comput. Biol., 4:e1000207.

Stoppin, V., Vantard, M., Schmit, A. C., and Lambert, A. M. (1994). Isolated plant nuclei nucleate microtubule assembly: the nuclear surface in higher plants has centrosome-like activity. Plant Cell, 6:1099.

Sugimoto, K., Williamson, R. E., and Wasteneys, G. O. (2001). Wall architecture in the cellulose-deficient rsw1 mutant of arabidopsis thaliana: Microfibrils but not microtubules lose their transverse alignment before microfibrils become unrecognizable in the mitotic and elongation zones of roots. Protoplasma, 215:172.

ten Hove, C. A., Lu, K. J., and Weijers, D. (2015). Building a plant: cell fate specification in the early arabidopsis embryo. Development, 142:420. 
Tindemans, S., Deinum, E., Lindeboom, J., and Mulder, B. (2014). Efficient event-driven simulations shed new light on microtubule organization in the plant cortical array. Frontiers in Physics, 2:19.

Tindemans, S. H., Hawkins, R. J., and Mulder, B. M. (2010). Survival of the aligned: Ordering of the plant cortical microtubule array. Phy. Rev. Lett., 104:58103.

Turing, A. M. (1952). The chemical basis of morphogenesis. Phil. Trans. Roy. Soc. B, 237:32.

Ubeda-Tomas, S., Beemster, G. T. S., and Bennett, M. J. (2012). Hormonal regulation of root growth: integrating local activities into global behaviour. Trends in Plant Science, 17:1360.

Ueng, S. K. and Sikorski, K. (1996). A note on a linear time algorithm for constructing adjacency graphs of 3d fea data. The Visual Computer, $12: 445$.

Uyttewaal, M., Burian, A., Alim, K., Landrein, B., Borowska-Wykret, D., Dedieu, A., Peaucelle, A., Ludynia, M., Traas, J., Boudaoud, A., Kwiatkowska, D., and Hamant, O. (2012). Mechanical stress acts via katanin to amplify differences in growth rate between adjacent cells in arabidopsis. Cell, 149:439.

Valster, A. H., Pierson, E. S., Valenta, R., Hepler, P. K., and Emons, A. M. C. (1997). Probing the plant actin cytoskeleton during cytokinesis and interphase by profilin microinjection. Plant Cell, 9:1815.

van den Berg, C., Willemsen, V., Hendriks, G., Weisbeek, P., and Scheres, B. (1997). Short-range control of cell differentiation in the arabidopsis root meristem. Nature, 390:287.

vonGuttenberg, H. (1964). Die entwicklung der wurzel. Phytomorph., 14:265.

Vos, J. W., Dogterom, M., and Emons, A. M. (2004). Microtubules become more dynamic but not shorter during preprophase band formation: A possible "search-and-capture" mechanism for microtubule translocation. Cell Motil. Cytoskeleton, 57:246.

Vos, J. W., Sieberer, B., Timmers, A. C. J., and Emons, A. M. C. (2003). Microtubule dynamics during preprophase band formation and the role of endoplasmic microtubules during root hair elongation. Cell. Biol. Int., $27: 295$. 
Wang, Q. and Huang, S. (2014). Visualization of microtubule organization and dynamics in living arabidopsis embryonic cells. Molecular Plant, $7: 1397$.

Wasteneys, G. O. (2002). Microtubule organization in the green kingdom: chaos or self-order? Journal of Cell Science, 115:1345.

Wasteneys, G. O. and Williamson, R. E. (1989). Reassembly of microtubules in nitella tasmanica: quantitative analysis of assembly and orientation. Eur. J. Cell Biol., 50:76.

Wendrich, J. R. and Weijers, D. (2013). The arabidopsis embryo as a miniature morphogenesis model. New Phytol, 199:14.

Willemsen, V., Bauch, M., Bennett, T., Campilho, A., Wolkenfelt, H., Xu, J., Haseloff, J., and Scheres, B. (2008). The nac domain transcription factors fez and sombrero control the orientation of cell division plane in arabidopsis root stem cells. Dev. Cell, 15:913.

Worden, N., Wilkop, T. E., Esteve, V. E., Jeannotte, R., Lathe, R., Vernhettes, S., Weimer, B., Hicks, G., Alonso, J., Labavitch, J., Persson, S., Ehrhardt, D., and Drakakaki, G. (2015). Cesa trafficking inhibitor inhibits cellulose deposition and interferes with the trafficking of cellulose synthase complexes and their associated proteins korrigan1 and pom2/cellulose synthase interactive protein1. Plant Physiology, 167:381.

Wright, A. J., Gallagher, K., and Smith, L. G. (2009). discordia1 and alternative discordia1 function redundantly at the cortical division site to promote preprophase band formation and orient division planes in maize. Plant Cell, 21:234.

Xu, J., Hofhuis, H., Heidstra, R., Sauer, M., Friml, J., and Scheres, B. (2006). A molecular framework for plant regeneration. Science, 311:385.

Yoshida, S., de Reuille, P. B., Lane, B., Bassel, G. W., Prusinkiewicz, P., Smith, R. S., and Weijers, D. (2014). Genetic control of plant development by overriding a geometric division rule. Dev. cell, 29:75.

Zumdieck, A., Lagomarsino, M. C., Tanase, C., Kruse, K., Mulder, B., Dogterom, M., and Jülicher, F. (2005). Continuum description of the cytoskeleton: Ring formation in the cell cortex. Phys. Rev. Lett., 95:258103. 


\section{English summary}

This thesis work aimed at a better understanding of the molecular basis of oriented cell division in plant cell. As, the efficiency of plant morphogenesis depends on oriented cell division, this work should contribute towards a fundamental understanding of the molecular basis of efficient plant morphogenesis.

In chapter 2, we describe a modelling framework that allows us to simulate microtubule dynamics on the surface of arbitrary shapes.

In chapter 3, we explain the parametrization of the simulations framework that meaningfully captures biological buffering of fluctuations in different microtubule dynamics parameters.

In chapter 4, we explore the generic role of microtubule regulatory effects such as shape anisotropy, edge-catastrophe and enhanced microtubule stabilization on the orientation of the microtubule array.

In chapter 5, through a combined approach of experimental observations of cell division patterns and simulation of microtubule dynamics, we describe a possible molecular basis of oriented cell division during Arabidopsis early embryogenesis.

In Chapter 6, we infer the necessity of incorporating anisotropic growth/stress response of microtubules towards understanding division plane orientation in the growing epidermal root cells of Arabidopsis.

In Chapter 7, we summarise how the research work described in this thesis, yields new insight in oriented cell division of plant cells. With this, we contribute to an enhanced understanding of efficient plant growth and morphogenesis. 


\section{Acknowledgements}

I deeply acknowledge my mother (and other family members) for giving me continuous encouragement in pursuing higher education. I want to reserve my gratitude for my teachers in my high school and colleges, for educating me with the basics of physics, mathematics and computer programming.

Ben ! you are an amazing person. From theoretical physics background, I never imagined to be working in a fundamental research problem of developmental biology. You are so much inspiring towards embedding of myself into the interdisciplinary research. Not a single day, I felt to be working in a laboratory with experimental biologists. I had the opportunity to see people from different scientific backgrounds, for me you are special and will remain so.

Bela ! I find myself extremely fortunate to have you as mentor for the computational work. To build the modelling framework, we tried so many different strategies that were failed. You always appreciated those endeavors as part of my training activity and encouraged me to try another one. "No body learn without getting it wrong (by sakira) ...", this is the message I am taking with me from you.

Viola, you deserve special thanks and acknowledgement. A significant part of our computational work is inspired by your experimental contribution in this thesis. Also Merijn, your involvement in image analysis accelerated the root epidermis project.

Ikram \& Renze, thanks for the critical suggestions on biologically relevant ideas in various occasions. Renze \& Hugo, I really appreciate your help in improving the appearance of the cover of this thesis. Sabine, thanks for all the help and support at the early phase of my PhD, you are the one who has the maximum influence on me among office colleagues.

Thanks to Ikram, Menno, Wenkun, Jorge, Alejandra, Luca, Jeroen and Rik for critical reading of the final version the various chapters of this thesis. I also thank all the other members of the Plant Developmental Biology Group (Wageningen UR).

I would like to thank the group of Marcel (Cell Biology, Wageningen UR), Pieter Rein (Biochemical Networks, AMOLF) and Bela (Theory of Biomolecular Matter, AMOLF), for giving me the opportunity to participate in their regular work discussions. I learnt about experimental cell biology, theory of biochemical networks and theoretical cell physics from these three groups. 


\section{Education Statement of the Graduate School Experimental Plant Sciences}

Issued to: Bandan Chakrabortty

Date: $\quad 30$ May 2017

Group: Plant Developmental Biology

University: Wageningen University \& Research

\section{1) Start-up phase}

- First presentation of your project

Title: Theoretical studies on gene regulatory networks during time varying perturbation

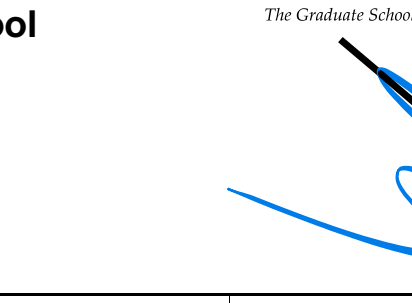

- Writing or rewriting a project proposal

Title: Simulating organ growth by combining gene networks and physical forces, and theoretical investigations on gene/protein networks during oscillatory perturbations

- Writing a review or book chapter MSc courses

- EPS theme symposia

Annual EPS Theme 1 symposium 'Developmental Biology of Plants', Wageningen, NL

Annual EPS Theme 1 symposium 'Developmental Biology of Plants', Leiden, NL

- Lunteren days and other National Platforms

Annual Meeting 'Molecular Gentics', Lunteren, NL

Annual Meeting 'Experimental Plant Sciences', Lunteren, NL

Dutch Biophysics Meeting, Veldhoven, NL

Annual Meeting 'Experimental Plant Sciences', Lunteren, NL

Annual Meeting 'Experimental Plant Sciences', Lunteren, NL

Dutch Biophysics Meeting, Veldhoven, NL

- Seminars (series), workshops and symposia

Symposium on Systems Biology for Food, Feed, and Health (WCSB), Wageningen

Meeting Plant Projects in VML of IPOP SB

WCSB Retreat

Symposium on Systems Biology for Food, Feed, and Health (WCSB), Wageningen

Annual Dutch Society for Developmental Biology Meeting, Utrecht, NL

European Embryogenesis Consortium Meeting

- Seminar plus

- International symposia and congresses

European Conference on Mathematical and Theoretical Biology, Nottingham, UK

Auxin Conference, Sanya, China

- Presentations

Poster: Dutch Biophysics Meeting, Veldhoven, NL

Talk: Annual Dutch Society for Developmental Biology Meeting, Utrecht, NL

Talk: European Embryogenesis Consortium Meeting

Poster: Dutch Biophysics Meeting, Veldhoven, NL

Nov 29, 2013

Jan 29-30, 2015

Jan 28-29, 2016

Jan 24, 2014

Jan 08, 2015

Oct 10-11, 2013

Apr 14-15, 2014

Sep 29, 2014

Apr 13, 2015

Apr 11-12, 2016

Oct 03-04, 2016

Jun 27, 2013

Jan 09, 2014

Feb 27-28, 2014

Jun 24, 2014

Apr 20, 2016

May 09-10, 2016

- IAB interview

- Excursions

Jul 11-15, 2016

Oct 20-25, 2016

Sep 29, 2014

Apr 20, 2016

May 09-10, 2016

Oct 03-04, 2016

Subtotal Scientific Exposure

14.9 credits $^{*}$

\section{3) In-Depth Studies}

- EPS courses or other PhD courses

NCSB Course on Basic Parameter Estimation

NCSB course on Modelling of Biological Processs

EMBO Practical Course: Microscopy, Modelling and Biophysical Methods, Heidelberg, Germany

- Journal club

Literature discussions at Wageningen UR and FOM Institute AMOLF

- Individual research training

\begin{tabular}{|c|c|}
\hline & $\underline{\text { date }}$ \\
& Jun 13, 2013 \\
Aug 01-07, 2013 & Sep 08-20, 2014 \\
& $2013-2016$ \\
\hline
\end{tabular}

\section{4) Personal development}

- Skill training courses

EPS PhD ExPectationS Day (career day), Wageningen, NL

WGS course 'Techniques for Writing and Presenting a Scientific Paper', Wageningen, NL

WGS course 'The Essentials of Scientific Writing and Presenting', Wageningen, NL

A course on Multi-scale Integration in Biological Systems, Paris, France

Subtotal In-Depth Studies

7.8 credits $^{*}$

- Organisation of PhD students day, course or conference

$\checkmark \quad$ Membership of Board, Committee or PhD council

date

Mar 28, 2014

Jul 01-04, 2014

Nov 24-25, Dec 01-02, 2014

Nov 13-18, 2014

Herewith the Graduate School declares that the PhD candidate has complied with the educational requirements set by the Educational Committee of EPS which comprises of a minimum total of 30 ECTS credits 
The research described in this thesis was financially supported by IPOP program of Wageningen University \& Research.

Computational work described in this thesis was carried out on the Dutch national einfrastructure with the support of SURF Foundation.

Financial support from the Plant Development Biology Group of Wageningen University, for printing this thesis is gratefully acknowledged.

Cover design by Bandan Chakrabortty 